\title{
Basics of Perturbative QCD
}

\author{
Yu. L. Dokshitzer \\ V. A. Khoze \\ A. H. Mueller \\ S. I. Troyan \\ EDITIONS \\ FRONTIERES
}

1991 


\section{Basics of}

\section{PERTURBATIVE QCD}

Yu. L. Dokshitzer, V. A. Khoze

A. H. Mueller and S. I. Troyan

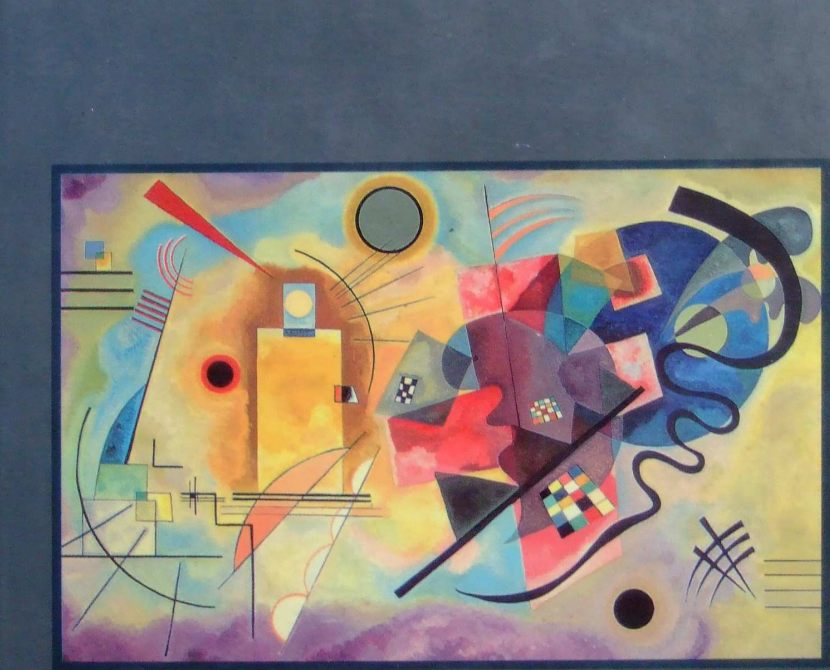

EDITIONS

FRONTIERES 


\section{"Basics of"}

a series edited by

J. Tran Thanh Van

Basics of Electron Positron Collisions

F. M. Renard

Basics of Lie Groups

M. Gourdin

\section{Basics of Cosmic Structures}

L. M. Celnikier

Basics of Modern Cosmology

A. D. Dolgov, M. V. Sazhin, Ya. B. Zeldovich

Basics of Perturbative QCD

Yu. L. Dokshitzer, V. A. Khoze, A. H. Mueller, S. I. Troyan

ISBN 2-86332-101-3 (hard cover)

ISBN $\quad 2-86332-102-1$ (soft cover)

ISSN $\quad 0982-5657$

Copyright 1991 by Editions Frontières

Printed in Singapore by Fong and Sons Printers Pte. Ltd 


\section{Contents}

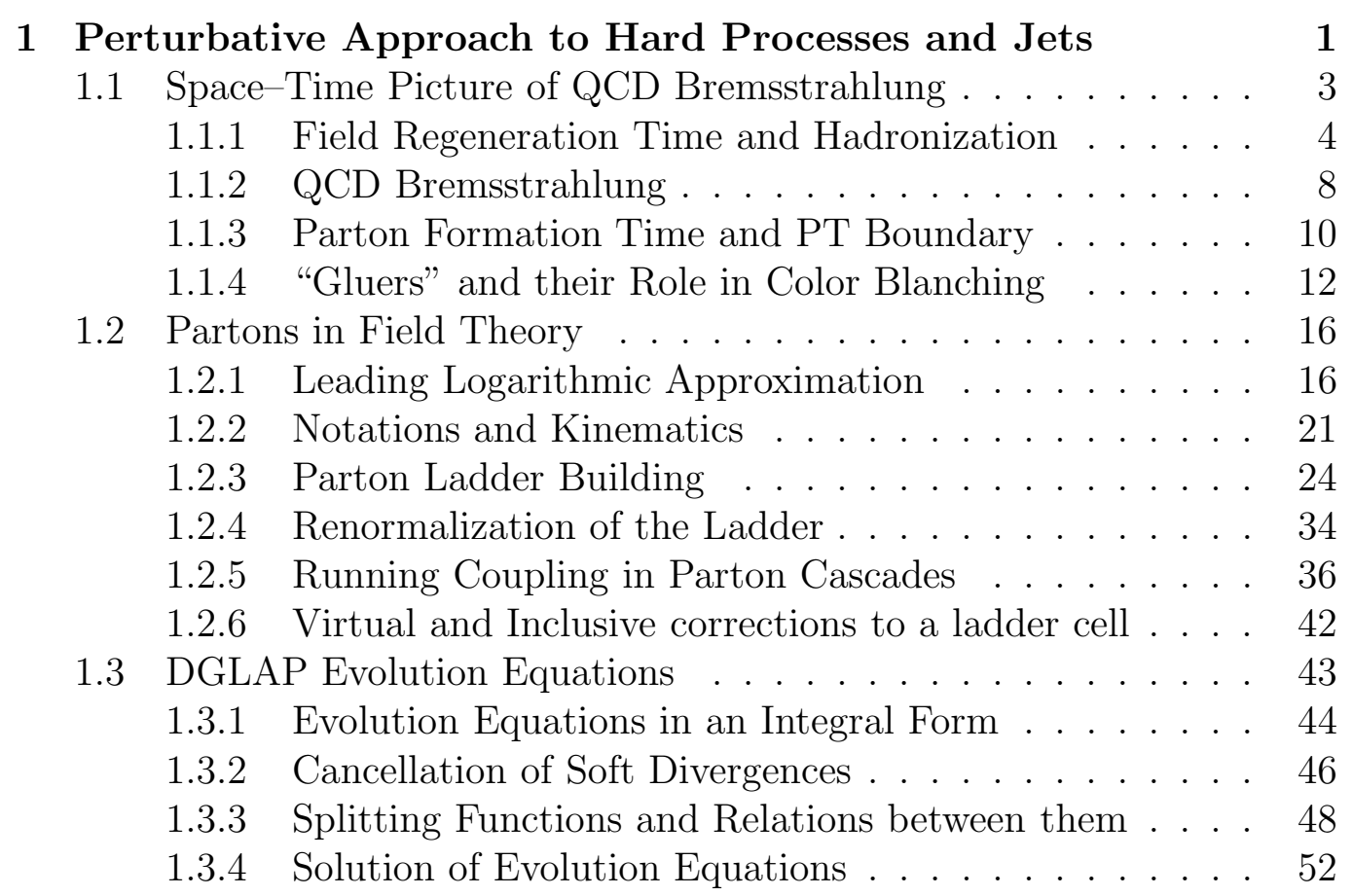


Bibliography . . . . . . . . . . . . . . . . . 62

2 Spin Dependent DIS and the Axial Anomalv 65

2.1 The Box Graph. the Triangle Graph and the OPE . . . . . . 66

2.1 .1 The Box Graph . . . . . . . . . . . . . . . . . 67

2.1.2 The Triangle Graph . . . . . . . . . . . . . . . . . . . 73

2.1.3 The Operator Product Expansion . . . . . . . . . . . 75

2.2 Partonic Interpretation of Spin Dependent DIS . . . . . . . 75

2.2.1 Phenomenological Definitions of Quarks and Gluons . . 76

2.2.2 Light-Cone Wave Function Definition of Partons . . . . 78

2.3 Parton and Constituent Quark Model of Hadrons . . . . . . . 81

2.4 The Experimental Situation . . . . . . . . . . . . . . . . . 85

Bibliography . . . . . . . . . . . . . . . . . 88

3 Exclusive Reactions and Color Transparency $\quad 89$

3.1 Review of Exclusive Reactions . . . . . . . . . . . . . . . 90

3.1.1 The Hard Scattering and Landshoff Pictures . . . . . . 91

3.1.2 The Sudakov Facton . . . . . . . . . . . . . . . . . 96

3.1 .3 The Elastic Form Factor . . . . . . . . . . . . . . . . . 99

3.2 Almost Exclusive Reactions in Nuclei . . . . . . . . . . . . . 101

3.2.1 Quasielastic Proton Scattering on Nuclei . . . . . . . . 101

3.2.2 Quasielastic Electron Scattering on Nuclei . . . . . . . 104

3.3 Evolution of Compact Quark Svstems . . . . . . . . . . . 105

3.3 .1 Classical Expansion . . . . . . . . . . . . . . . 106

3.3.2 Quantum Expansion . . . . . . . . . . . . . . . . . 108

Bibliography ........................... 111

4 Guide to Color Coherence 113

4.1 Angular Ordered Parton Cascades . . . . . . . . . . . . . . . 114 
4.2 Angular Ordering in Initial State Radiation . . . . . . . . . 117

4.3 The Origin of Hump-Backed QCD Plateau . . . . . . . . . . . 123

4.4 Radiation pattern for the $q \bar{q}$ Antenna . . . . . . . . . . . . . 126

4.5 Wave Nature of Drag Effects . . . . . . . . . . . . . . . . . . 129

4.6 Two-particle Correlations . . . . . . . . . . . . . . . . 132

4.7 Fevnman-Gribov Puzzle and QCD Coherence . . . . . . . . . 134

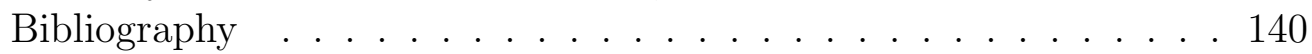

5 Double Logarithmic Approximation 143

5.1 Tree Multigluon Amplitudes for $e^{+} e^{-} \rightarrow q \bar{q}+N g$. . . . . . . . 145

5.1.1 Two Gluon Emission off a Quark . . . . . . . . . . . . 147

5.1.2 Angular Ordering for $N=2$. . . . . . . . . . . . . 150

5.2 Angular Ordering in All Orders . . . . . . . . . . . . . . . . . 152

5.3 Virtual Corrections . . . . . . . . . . . . . . . . . . . . 157

5.4 Method of Generating Functionals . . . . . . . . . . . . . . . . 158

5.4.1 Exclusive and Inclusive Cross Sections . . . . . . . . . 159

5.4.2 Generating Functional (GF)s for $q$ and $q$ Jets . . . . . 160

5.5 Multiplicity Distributions in QCD Jets . . . . . . . . . . . . 165

5.6 KNO Phenomenon .................... . . 170

5.6.1 Recurrency Relations for Multiplicity Moments . . . . 172

5.6.2 Analvtic Solution of the KNO Problem . . . . . . . . . 174

5.7 Particle Distributions and Correlations . . . . . . . . . . . . . 177

5.7.1 Energy Spectrum of Particles in Jet . . . . . . . . . . . 178

5.7.2 Correlations Between Particles in Jet . . . . . . . . . . 180

Bibliography ...................... 185

6 Modified Leading Logarithmic Approximation 187

6.1 Single Logarithmic Corrections to DLA Cascades . . . . . . . 188

6.2 MLLA Evolution Equations for Intrajet Cascades . . . . . . . 195 
6.3 Pattern of Multiple Gluon Bremsstrahlung . . . . . . . . . . . . 199 6.3.1 The BCM Ansatz and the Dipole Scheme . . . . . . . . 199

6.3.2 Construction of the Probabilistic Scheme . . . . . . . . 201

6.3.3 Jet Polarizability and Color Monsters . . . . . . . . . . 213

6.3.4 Magnitude of Dipole Corrections . . . . . . . . . . . . 218

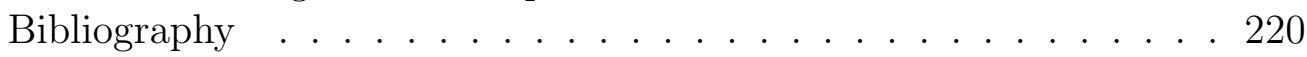

7 MLLA Hump-Backed Plateau 221

7.1 Evolution Equation for Particle Spectra . . . . . . . . . . . . . 222

7.2 Analvtic Solution . . . . . . . . . . . . . . . . . . 225

7.3 Developed Cascade and LPHD Hvpothesis . . . . . . . . . . . 229

7.4 Mean Parton Multiplicity . . . . . . . . . . . . . . . . . . 233

7.5 Shape of the Limiting Spectrum . . . . . . . . . . . . . . . . . 235

7.5.1 Gaussian Approximation . . . . . . . . . . . . . 235

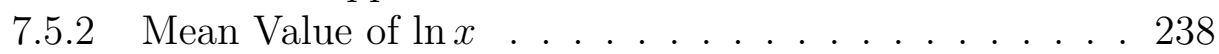

7.5.3 Width of the Hump . . . . . . . . . . . . . . . . . . . 239

7.5.4 Skewness and the Peak Position . . . . . . . . . . . . . 241

7.6 Evaluation of the Limiting Spectrum . . . . . . . . . . . . . . 242

7.7 Preexponential Corrections to the Spectrum . . . . . . . . . . 243

7.8 Spectra in Jets with Restricted Opening Angles . . . . . . . . 246

7.9 Phenomenology of Hump-Backed Plateau . . . . . . . . . . . 249

7.9.1 Mean Particle Multiplicities . . . . . . . . . . . . . . . 249

7.9.2 Inclusive Particle Spectra . . . . . . . . . . . . . . . . 251

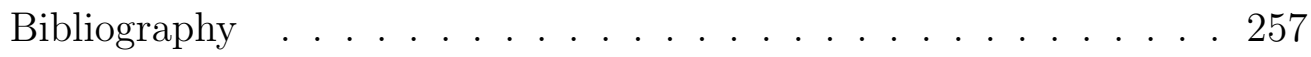

8 The Physical Picture of Shadowing in Nuclei 259

8.1 Introduction . . . . . . . . . . . . . . . . . 260

8.2 Small $x$ Lepton-Nucleus DIS in the Parton Model . . . . . . . 262

8.2.1 The Infinite Momentum Frame . . . . . . . . . . . . . 262 
8.2 .2 The Laboratorv Frame . . . . . . . . . . . . . . . . 264

8.3 Deeplv Inelastic Scattering in QCD . . . . . . . . . . . . 266

8.3.1 Infinite Momentum Frame . . . . . . . . . . . . . 267

8.3 .2 The Laboratorv Svstem . . . . . . . . . . . . . . 267

8.4 Dilute Versus Dense Domains of Partons . . . . . . . . . . . 270

8.5 OCD Phenomenology of Shadowing . . . . . . . . . . . . 271

8.6 Massive $\mu$-Pairs from Hadron-Nucleus Collisions . . . . . . . . 273

Bibliography . . . . . . . . . . . . . . . . 277

9 Radiophysics of QCD Jets 279

9.1 On Structure of Particle Flows in Multijet Events . . . . . . . 281

9.2 QCD Portrait of an "Individual Jet" . . . . . . . . . . . . 284

9.2.1 Collimation of Energy in Jet . . . . . . . . . . . . . 285

9.2.2 Enerov Spectrum Within a Given Cond . . . . . . . . . 289

9.2.3 Collimation of Multiplicitv Inside Jet . . . . . . . . . . 293

9.3 Multiplicitv Flow Pattern for $q \bar{a} a$ Ensemble . . . . . . . . . . 294

9.3.1 Radiation Pattern for $q \bar{\sigma} \gamma$-events . . . . . . . . . . 296

9.3.2 Radiation Pattern for $q \bar{q} q$-events . . . . . . . . . . . . 299

9.3.3 Average Multiplicitv of 3-iet events . . . . . . . . . . . 301

9.4 QCD Drag Effect in Interjet Particle Flows . . . . . . . . . 306

Bibliography . . . . . . . . . . . . . . . . . 318

10 Collective Color Phenomena in Hadronic Scattering 321

10.1 QCD Coherence in High- $p$, Collisions . . . . . . . . . . . 322

10.2 Color Transfer and Particle Flows . . . . . . . . . . . . . . 324

10.3 Two-Jet Production at Large $p_{1} \ldots \ldots \ldots$. . . . . . . . . . 328

10.4 Prompt $\gamma . W . Z$ Production at Large $p \ldots \ldots 334$

10.5 Color Structure of Heavv Ouark Production . . . . . . . . . 339

10.6 Azimuthal Asvmmetrv of QCD Jets . . . . . . . . . . . 343 
viii

10.6.1 Jet Asvmmetrv in $e^{+} e^{-}$annihilation . . . . . . . . . . 344

10.6.2 Jet Asvmmetrv in hadron scattering . . . . . . . . . . 348

10.7 Lessons . . . . . . . . . . . . . . . . . . . 353

Appendix: Radiation in Parton-Parton Scattering . . . . . . . . . . 358

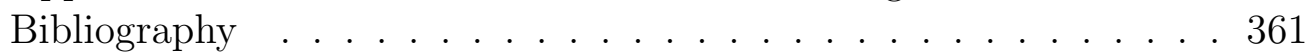

\begin{tabular}{ll}
\hline Index. Abbreviations & 362
\end{tabular} 


\section{Prologue}

Our modern understanding of the high energy behavior of strong interactions was conceived with the idea of the parton model in the late 1960's and was born with the appearance of asymptotic freedom in the early 1970's. Partons arose out of a necessity to explain the scaling observed in deep inelastic electron scattering experiments at SLAC. This phenomenological understanding of SLAC scaling was soon extended to other hard scattering processes including $e^{+} e^{-}$annihilation into hadrons and inclusive high $p_{\perp}$ hadron production in hadron-hadron collisions. However, the idea of what exactly a parton was remained elusive and the phenomenological successes of the parton model remained qualitative rather than quantitative.

With the coming of asymptotic freedom in nonabelian gauge theories in the 1970's it became apparent that partons were nothing other than the quanta which occur in the theory of quark and gluons interacting by means of the gluon couplings to color charge, QCD. Asymptotic freedom allows one to consider quarks as free quanta at short distances and over short times thus making contact with the idea of a parton as a noninteracting constituent of a hadron. It soon became clear that for many, if not all, high energy processes involving a large momentum transfer one could separate (factorize) the process into one part which involves only hard interactions and which is 
calculable using perturbative QCD and into a second part which requires detailed nonperturbative information as to how hadrons are built out of quarks and gluons. The parts of hard processes involving nonperturbative physics are not energy dependent and can be used in one process after having been measured in another process.

Our understanding of partons in QCD has progressed rapidly since the 1970's and now QCD partons are used both quantitatively and qualitatively to explain diverse phenomena in both inclusive and exclusive high energy reactions. Sometimes their applications are rather simple conceptually as for example in calculations involving higher order corrections to the electronpositron annihilation cross section into hadrons. Sometimes techniques far beyond traditional renormalization group methods are required as for example in our description of inclusive hadron production in jets. Sometimes it requires great care in deciding what exactly counts as a parton and when a parton counts as a quark and when it counts as a gluon as for example in our discussion of spin-dependent deep inelastic scattering. Sometimes it is not yet clear what are the limits of the usefulness of the parton picture as for example in very small $x$ phenomena.

In this book our approach is completely perturbative. We make no attempt to describe hadron wavefunctions in terms of quarks and gluons. Much of this book is concerned with distributions and correlations of particles produced in, or in association with, jets. However, phenomenologically distributions of hadrons and of partons seem remarkably similar. This has led to hypothesis of local parton hadron duality (LPHD). LPHD has made it possible to apply the predictions of perturbative QCD at much lower energies than would have been the case had the relationship between partons and hadrons not been so close.

If there is a main focus to this book it is obtaining high energy predictions of QCD in circumstances where coherence is important. Little of our discus- 
sion is untouched by the idea that gluons couple to quarks and other gluons by means of a conserved color charge. Some of the predictions discussed in this book are at first sight anti-intuitive as for example the look of a central plateau in jets. Indeed the emergence of the "hump-backed" plateau shape of the inclusive hadron spectrum in jets, recently definitely confirmed at LEP, is perhaps the most striking example of a highly nontrivial prediction of QCD where coherence plays a predominant role. It is coherence, also, which allows the evolution of a jet from a single parton into a multiparton system to be described, in most of its aspects, in terms of a classical branching process, which description has been so important for understanding the global structure of events in $e^{+} e^{-}$and hadron-hadron collisions. Color coherence can be used practically as a valuable tool for studying manifestations of new physics. For example, reconstruction of the QCD radiation pattern determinig distributions of accompanying hadrons may help to explore the production of new heavy objects: the Higgs bosons, new quarks, supersymmetric particles, etc.

In describing particle distributions and correlations in jets for that region of phase space where most of the particles are produced an unusual circumstance arises. The perturbation theory does not just consist of a few Feynman graphs giving a few powers of $\alpha_{s}$, rather both single and double logarithms occur for each power of $\alpha_{s}$. This necessitates a resummation of the perturbation series. The summation of all double logarithmic terms, $\left[\alpha_{s} l^{2}\right]^{n}$, which we refer to as DLA gives a good qualitative description of inclusive distributions in jets, however, for a quantitative description it is necessary to include single logarithmic terms leading to the modified leading logarithmic approximation, MLLA. In MLLA distributions are generally down by $\sqrt{\alpha_{s}}$ compared to DLA. The appearance of $\sqrt{\alpha_{s}}$ corrections is one of the surprising features of much of the physics we discuss in this book. $\sqrt{\alpha_{s}}$ terms arise because in the process of summing leading double logs we neglect quantities which have summed up decreasing exponentials $\exp \left(-\sqrt{\alpha_{s}} \ln \right)$ while we keep increasing 
exponentials of $\sqrt{\alpha_{s}} \ln$. Dropping these decreasing exponentials leads to an expansion in $\sqrt{\alpha_{s}}$ an expansion which has now been reasonably well tested at LEP.

This book is aimed at two rather different audiences. Much of our discussion is descriptive and large parts of the book can be read without going through the details of the derivations of the final results. Thus one of our objectives is to make the book accessible to high energy experimental physicists. We have tried to explain the various phenomena in physical terms and we have stated results often in a directly usable way. On the other hand we have tried to be complete enough in our technical discussions so that the derivations can be followed by an advanced graduate student. Little of what we cover is available in book from elsewhere.

Experimental results are coming in at a very rapid rate, especially from LEP. In order that this book should not age too rapidly we have kept comparisons with experiment to a minimum. We have tried to concentrate on the ideas and on the precise predictions of QCD. Most of the topics we have covered are reasonably mature from a theoretical perspective, though often definitive experimental tests are yet to come.

In the long course of preparing this book we benefited a lot from collaboration with many of our colleagues belonging to the QCD community.

We are especially indebted to B. Andersson, Ya.I. Azimov, S. Bethke, G. Cowan, V.S. Fadin, V.N. Gribov, G. Gustafson, G. Marchesini, P. Mättig, C. Peterson, T. Sjöstrand and B. Webber for fruitful discussions.

This work was completed during the visit of three of us (TDK) at Lund University in the framework of the Nordita Perturbative QCD Workshop. It is a pleasure for us to thank the Theory Group of Lund University for having arranged a nice time for physics there. 


\section{Chapter 1}

\section{Perturbative Approach to Hard Processes and Jets}

\section{Contents}

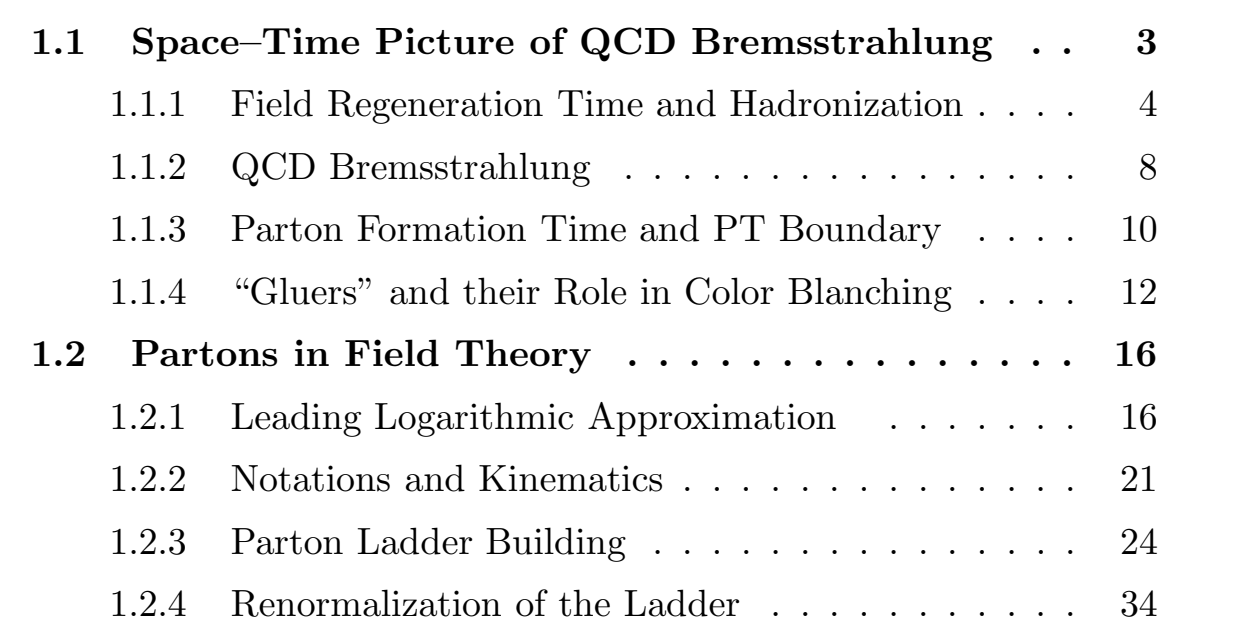




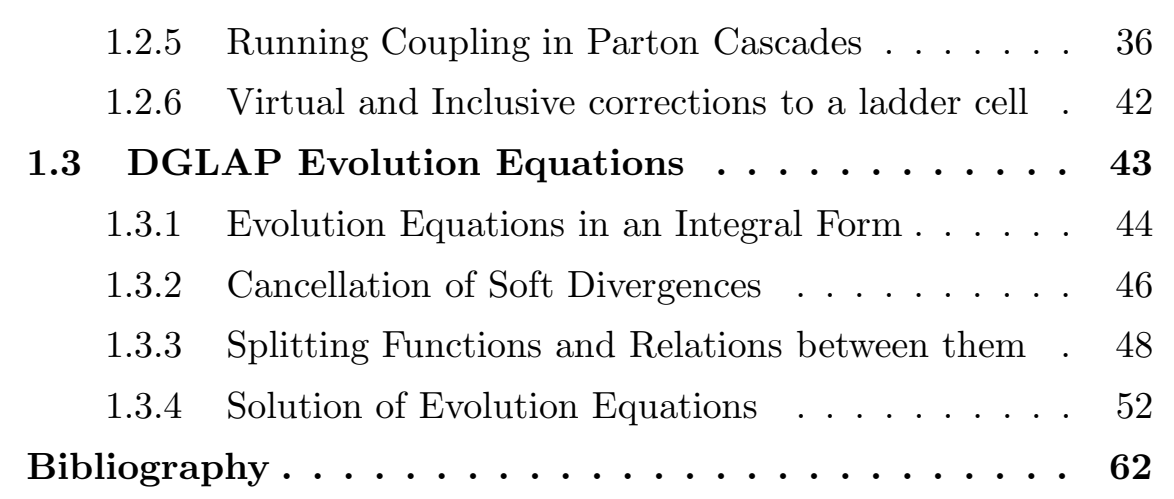

Perturbative (hereafter - PT) QCD aims to describe quantitatively the structure of multipartonic systems produced by QCD cascades for gaining some actual knowledge about confinement from comparing the calculable characteristics of quark-gluon ensembles with measurable characteristics of final hadronic states in hard processes.

It has become a matter of folklore to say that QCD has taken over from the old Parton Model. This is however only a half truth. Indeed, if one is thinking of the Hard Processes such as $e^{+} e^{-} \rightarrow$ hadrons or deeply inelastic lepton-hadron scattering (DIS) this is just the case: it was QCD that supplied the heuristic parton picture with the dynamical basis. At the same time it would be wasteful to forget that the main advantage of the Parton Model 1 was just a universal approach to both "hard" and "soft" physics. The parton idea has explained the phenomenon of scaling in structure functions (hard DIS and $e^{+} e^{-}$processes) and in the inclusive particle distributions in hadron collisions at the same time. It was the parton picture which predicted a jet-like structure of final states of hard interactions and, on the other hand, which gave a clear explanation of Regge asymptotics 2 . 
Modern QCD is far from reaching such a universality, the impassable infrared gulf still lies between soft and hard processes. A characteristic of the QCD burst of the last 15 years - certain coolness to a class of problems of Reggistics, does not mean a nonurgency of the topic. Simply it remains to be too hard a nut to crack for the modern theory.

Meanwhile the need of an integrated approach to the description of both hard and soft hadroproduction processes becomes imminent. It is the experimentally observed universality of quark distributions and jets, similar particle content in the hard lepton-hadron and soft hadron-hadron collisions etc. which point to this need.

To elaborate such an integrated approach one has to gain better understanding of the confinement mechanism, to search for more detailed qualitative and quantitative information about the region of really strong interaction where the quark-gluon language becomes inapplicable and starting from the QCD field Lagrangian we are unable to keep track of the happenings. To enter the problem we should start with portraying the space-time picture of the hadroproduction in hard processes i.e. drawing the picture of "blanching" of colored partons - quarks and gluons.

\subsection{Space-Time Picture of QCD Bremsstrahlung}

Now we proceed to the consideration of the basic hard interaction, namely the creation and propagation of a quark $q$ and an antiquark $\bar{q}$ in the annihilation process $e^{+} e^{-} \rightarrow q \bar{q}$.

The key problem here is how to organize a color neutral final system of "white" hadrons from the initial pair of colored quarks flying apart with 
relativistic velocities from the annihilation point. At first sight it seems to be rather difficult (if not even impossible) to reconcile the fast spreading of color with the final color neutrality needed. How to let quarks know that they should not take away color (and fractional electric charges by the way)?! Here we'll concentrate on this puzzle which furnishes the very core of the hadronization problem.

Firstly let us remind the reader a standard answer to the above question: "there exists the non-perturbative "gluon string" or the "flux tube" of strong chromomagnetic field between quarks (color charges) which starts to stretch and breaks producing new q $\bar{q}$ pairs to form hadrons subsequently. This incantation however has little to do with the problem under interest. Why so? Let us catch sight of this point.

The concept of the string (the "area law" in the Wilson loop, the linearly rising potential and all that) could in principal explain "why do quarks form a white hadron" but not "how does this confinement occur in the fast non-adiabatic process". The reason for this is that relativistic quantum mechanics (together with asymptotic freedom) protects the fast quark from being involved in any non-PT interaction during a long time. The original $q$ and $\bar{q}$ come out of the PT jurisdiction and can enter the "hadronization game" only after macroscopically large time interval from the start of the process, which is proportional to the quark energy: $\quad t_{\text {hadr }} \propto E$.

\subsubsection{Field Regeneration Time and Hadronization}

The essence of a "hard process" is that the quark is knocked out from the vacuum (or from a hadron as in DIS process) as a bare particle or, more accurately, as a half-dressed one. This means that the charge, when being accelerated, appears to have a truncated proper field (either electro- or

chromo-magnetic). Its field has no Fourier components with $k_{\perp}<\sqrt{Q^{2}}$ 
where $Q^{2}$ denotes the characteristic momentum transfer squared measuring the "hardness" of the hard process.

Subsequently two closely correlated processes start: the bremsstrahlung is developing whose quanta in time leave the parent radiating quark as offspring partons - gluons, and the private gluonic field of the quark is regenerated. The phenomenon of regeneration of a stationary field surrounding a charge has been understood in QED. The regeneration time of the proper field or, to be more precise, of its Fourier component with momentum $\vec{k}$ is given by

$$
T_{\text {regen. }}(k) \sim \frac{k_{\|}}{k_{\perp}^{2}},
$$

where longitudinal and transverse components of photon momentum are defined with respect to the outgoing electron. For photons with relatively small transverse momenta $k_{\perp} \ll k_{\|} \sim p \approx E$ where $p$ and $E$ stand for electron momentum and energy, the regeneration time may become macroscopically large. The finiteness of the regeneration time leads to substantial effects for relativistic electrons.
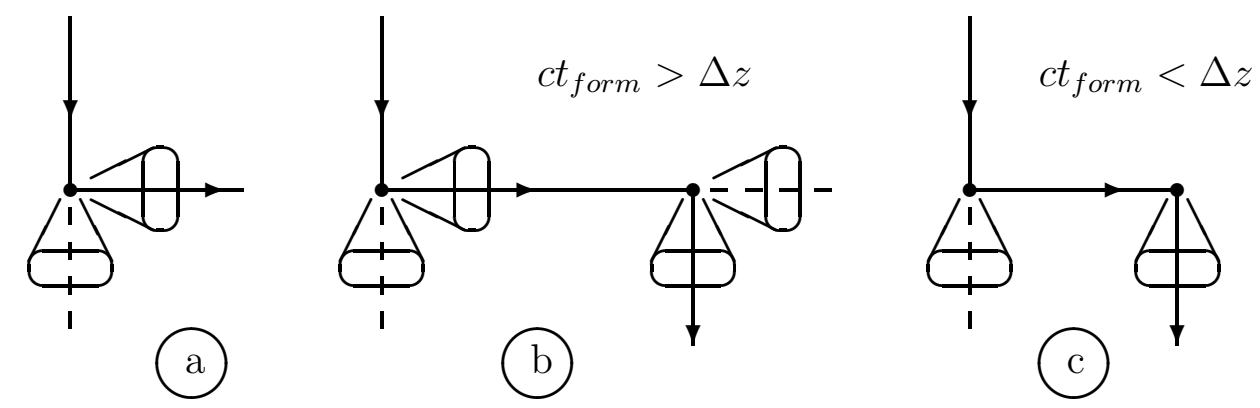

Figure 1.1: Photon bremsstrahlung accompanying electron scattering. 
When the fast electron is scattered at large angle, two cones of bremsstrahlung photon radiation are well known to be formed (see Fig. 1.17). The quanta from the first cone centered around the direction of an initial electron momentum can be treated as splinters of an electromagnetic surrounding shaken off by the accelerated electron. An appearance of the second cone is the back of the regeneration of a new field coat i.e. the disk of the Lorentz contracted Coulomb field fitting the final electron.

Next, for a double scattering process depicted in Fig. 1.1b it might seem natural to expect an appearance of the four photon cones. However if the regeneration length $c T_{\text {regen. }}$ (1.1) exceeds the distance between the scattering points, only two of them actually emerge (Fig. 1.1k).

The regeneration physics shows up in a number of QED phenomena connected with relativistic electrons, such as the Landau-Pomeranchuk damping of soft bremsstrahlung due to multiple rescatterings of electron in a medium, in transmission radiation, electron radiation in crystals etc.

Classical considerations alone give evidence of a truncated field. Let us take the classical charge which is moving along $z$ axis with velocity $v \approx 1$ after being accelerated (say, from a $v=0$ state) at $t=0$. At asymptotically large time it will be surrounded by a disk of Lorentz contracted e.m. field. It is clear however that such a state could not emerge instantly. In the reference frame accompanying the charge the field spreads out inside the sphere

$$
r^{\prime} \leq t^{\prime}
$$

This means that in the laboratory frame where the time is slowered by the factor $\gamma=E / m$, the field at distances $r$ from apart the $z$ axis will appear not earlier then at

$$
t=\gamma t^{\prime}=\frac{E}{m} r .
$$


Applied to a quark this results in a rather serious consequence: an energetic "bare" quark prepared by a hard interaction will be able to hadronize (i.e. to become a hadron constituent) only after the time (1.2), where $r$ measures typical value of interquark distances inside a hadron (hadronic size $R$ ) and $m$ should be treated as its constituent mass. For a light quark $(q=u, d, s)$ these two parameters are closely linked to each other and to the value of mean transverse momenta characteristic for soft hadron physics:

$$
m_{\text {constituent }} \sim \sqrt{\left\langle k_{\perp}^{2}\right\rangle} \sim R^{-1} \approx \text { few hundred } \mathrm{MeV}
$$

Thus for light and heavy $(Q=c, b, \ldots)$ quarks one arrives at the following estimates of hadronization time:

$$
\begin{aligned}
t_{q}^{\text {hadr }} & \approx E R^{2}, \\
t_{Q}^{\text {hadr }} & \approx \frac{E}{m_{Q}} R .
\end{aligned}
$$

The same conclusions could be drawn from

Quantum-Mechanical arguments. Indeed, in the rest frame of a hadron the confining forces stem from "long-wave" gluonic field with momentum components

$$
k_{\perp}^{\prime} \sim k_{\|}^{\prime} \sim k^{\prime} \sim R^{-1} .
$$

Moving back to the Lab. system one gets

$$
k_{\perp}=k_{\perp}^{\prime} \sim R^{-1}, \quad k_{\|}=\gamma k_{\|}^{\prime}=\frac{E}{m R},
$$

and the time (1.2) one needs for such a field to be regenerated reads:

$$
T_{\text {regen. }}(k)=\frac{k_{\|}}{k_{\perp}^{2}} \approx \frac{E}{m R} R^{2}=\frac{E}{m} R,
$$


which coincides with the classical formula (1.5).

Thus a quark with energy $E \sim 200 \mathrm{GeV}$ starting from the annihilation time $t^{a n n .} \sim 1 / E \sim 10^{-3} \mathrm{fm} / c$ and up to hadronization time $t^{\text {hadr }} \sim E R^{2} \sim$ $10^{3} \mathrm{fm} / c$ should behave as a true color particle radiating gluons perturbatively without any care taken about its future confinement. An instructive lesson comes from considering an ultraheavy quark $Q$ heavier than the mass of the weak boson $W: m_{Q}>100 \mathrm{GeV}$. Due to the semiweak decay $(Q \rightarrow W+q)$ its lifetime $\tau_{Q}$

$$
\tau_{Q} \approx 1 \mathrm{fm} / c\left(\frac{M_{W}}{m_{Q}}\right)^{3} \frac{E_{Q}}{m_{Q}}<t_{\text {hadr }} \approx 1 \mathrm{fm} / c \frac{E_{Q}}{m_{Q}}
$$

is shorter than the hadronization time so that for all its life it remains under the jurisdiction of PT QCD. One can say that such a quark in all aspects behaves as if it were a free colored object.

\subsubsection{QCD Bremsstrahlung}

Now, after touching upon the field regeneration physics we turn to bremsstrahlung gluon radiation. The differential spectrum of gluon radiation off a quark differs from the ordinary spectrum of a photon emitted by an electron only by the color factor $C_{F}=\left(N_{C}^{2}-1\right) / 2 N_{c}=4 / 3$ and, of course, by the substitution $\alpha \rightarrow \alpha_{s}=g_{s}^{2} / 4 \pi$ :

$$
d w^{q \rightarrow q g}=\frac{\alpha_{s}\left(k_{\perp}^{2}\right)}{4 \pi} 2 C_{F}\left[1+\left(1-\frac{k}{E}\right)^{2}\right] \frac{d k}{k} \frac{d k_{\perp}^{2}}{k_{\perp}^{2}},
$$

where $k_{\mu}$ is the 4-momentum of the gluon. The effective coupling here runs with the gluon transverse momentum $k_{\perp}$, which comes from higher order corrections to the Born probability. 
Let us notice two important properties of the spectrum (1.6). They are:

- broad logarithmic distribution over transverse momentum which is typical for a field theory with dimensionless coupling (high probability of quasicollinear $q g$ configurations) and

- broad logarithmic energy distribution specific for theories with massless vector bosons 1 .

Key words which one meets with in connection with these basic properties of the QCD bremsstrahlung phenomena are

- transverse logs, collinear divergency, mass singularity etc.,

- longitudinal logs, soft divergency, infrared singularity etc.

Picking up a gluon with large emission angle and large energy one would get an extra gluon jet with a small probability

$$
\text { Multi-Jet Events: } \quad k_{\perp} \sim k \sim E \quad \rightarrow \quad w \sim \frac{\alpha_{s}}{\pi} \ll 1 .
$$

In the same time the bulk of radiation (quasicollinear and/or soft gluons) will not lead to the appearance of additional visible jets in an event but will instead populate the original quark jet with secondary partons influencing the particle multiplicity and other jet properties

$$
\text { Intrajet Activity: } \quad k_{\perp} \ll k \ll E \quad \rightarrow \quad w \sim \frac{\alpha_{s}}{\pi} \log ^{2} E \sim 1 .
$$

Properties of the expression (1.6) for $q \rightarrow q g$ emission together with similar formulae for two other basic parton splittings $g \rightarrow g g$ radiation and $g \rightarrow q \bar{q}$ decay will be discussed in details below in Section 1.3.3.

\footnotetext{
${ }^{1}$ Massive vector particles such as $W$ and $Z$ will also exhibit logarithmic bremsstrahlung spectra at very high energies far above the weak mass scale.
} 


\subsubsection{Parton Formation Time and PT Boundary}

To answer the question as how do offspring partons influence the hadronic yield, one has to realize first what is the condition for a gluon to behave as an independent colored object and thus as an additional source of new particles. It takes some time to emit a gluon. This time (so called formation time) can be simply estimated as a life-time of a virtual $(p+k)$ quark state in Fig. 1.2 Making use of the Heisenberg uncertainty principle with account of

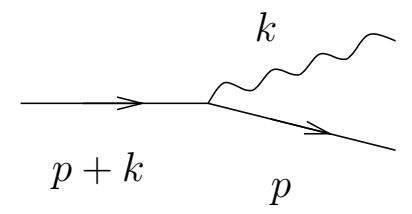

Figure 1.2: Kinematics of gluon emission.

the Lorentz contraction effect one arrives at

$$
t_{g}^{\text {form }} \sim \frac{1}{M_{\text {virt. }}} \frac{E}{M_{\text {virt. }}}=\frac{E}{(p+k)^{2}} \approx \frac{E}{k E \Theta^{2}} \approx \frac{k}{k_{\perp}^{2}} .
$$

Comparing this expression with that for the hadronization time of a gluon $k$ (cf. (1.4) )

$$
t_{g}^{h a d r} \approx k R^{2},
$$

one concludes that it is the transverse momentum restriction

$$
k_{\perp}>R^{-1}=\text { a few hundred } \mathrm{MeV}
$$

which guarantees the gluon's being since it seems reasonable to be born before ones death:

$$
t_{g}^{\text {form }} \sim \frac{k}{k_{\perp}^{2}} \quad<\quad t_{g}^{\text {hadr }} \sim k R^{2} .
$$


The same reasonings can be applied to any parton splitting process, so that the basic restriction (1.9) represents the condition of the very applicability of quark-gluon language or, in other words, of the PT QCD.

Notice that there exists another way of argumentation which approves this restriction as a natural limit for the PT approach. Namely, at finite values of $k_{\perp}$ the running coupling $\alpha_{s}$ in the expression (11.6) for the emission probability approaches the infrared pole, thus endangering PT considerations.

The boundary condition (1.9), being physically very important, leaves at the same time rather broad room for PT analysis. In collisions with large characteristic momentum transfer $Q$ the bulk of phase space where basic partonic amplitudes appear to be large (logarithmic; cf. (1.6))

$$
R^{-1} \ll k_{\perp} \lesssim k \lesssim \sqrt{Q^{2}}
$$

remains in the regime of $\mathrm{PT} \mathrm{QCD}$.

The parameter $\left(k_{\perp} R\right)$ can be said to regulate the "lifetime" of a secondary parton. Gluons with momenta satisfying the strong inequality

$$
k_{\perp} R \gg 1,
$$

which are the main characters of the PT play, will live for a long time radiating, in their turn, new offspring thus starting the cascade multiplication of partons.

To gain a large total emission probability in spite of $\alpha_{s}$ being small, one has to pick up at least one logarithmic integration out of the PT phase space (1.11). The list of possible orderings of items in (1.11) each of which reveals itself in definite physical phenomena look as follows.

- $R^{-1} \ll k_{\perp} \ll k \sim \sqrt{Q^{2}}$

That is quasicollinear hard parton splittings leading to the known scaling violation effects in DIS cross section and $e^{+} e^{-}$inclusive particle distributions. 
- $R^{-1} \ll k_{\perp} \sim k \ll \sqrt{Q^{2}}$

Large angle soft gluon emission responsible for drag effects in interjet multiplicity flows manifesting QCD Coherence.

- $R^{-1} \ll k_{\perp} \ll k \ll \sqrt{Q^{2}}$

Double-Logarithmic (soft \& collinear) gluon bremsstrahlung off quarks and gluons causing jet multiplicity growth with energy and determining QCD Form Factors of partons.

Before turning to the comprehensive study of different aspects of complicated multiparton states we'll make first a short stop for some "handwaving" discussion of the nature of blanching and hadronization phenomena.

\subsection{4 "Gluers" and their Role in Color Blanching}

Though disregarding parton pairs with small relative transverse momenta $k_{\perp}<R^{-1}$ which definitely lie beyond the scope of PT, let us concentrate for a moment on a role played by the gluons radiated at the lower edge of PT phase space (1.9) in the process of Fig. 1.2. To be serious, nothing can be said about the fate of these guys quantitatively in the PT framework. They are radiated strongly, $\alpha_{s}\left(k_{\perp}^{2}\right) \sim 1$, and can be hardly treated as gluons even, since due to relation (1.10) they are forced to hadronize just immediately after being formed. For the sake of definiteness let us call such an object

$$
\text { gluer : } \quad k_{\perp} R \sim 1,
$$

stressing the point that it is the prerogative of Gluers and not of Gluons to glue. 
Blanching $q \bar{q}$ Jets. An appearance of gluers is a signal of switching on the real strong interaction in a system of color charges. According to (1.7) first gluers are formed at $t \approx R$ with $k \sim k_{\perp} \sim R^{-1}$. It is the moment when the distance between the outgoing $q$ and $\bar{q}$ starts to exceed $1 \mathrm{fm}$.

What non-PT phenomenon has to happen at this moment? The answer should be the separation of two jets as globally blanched subsystems. Such a blanching is needed pragmatically to restrain the gluer radiation probability which otherwise should grow manifesting the infrared instability of the theory via the catastrophic increase of $\mathrm{PT}$ interaction strength $\alpha_{s}$.

Though up to now one has no quantitative description of blanching process, the plausible picture of what is going on can be extracted from the Gribov confinement scenario 3 , where the strong external field created by outgoing quarks causes the crucial restructuring of the light quark Dirac sea resulting in a phenomenon which is similar to the known QED physics of "supercharged ions" with $Z>137$.

Gluers and Hadron Plateau. With increasing time gluers with larger and larger energies $k \sim t / R^{2}$ are formed. If there were no PT gluon radiation at all, the resulting hadronic spectrum could be simply related to the integrated probability of emission of gluers which according to (1.6) gives the following qualitative estimate:

$$
d N=\left[\int_{k_{\perp} \sim R^{-1}} \frac{d k_{\perp}^{2}}{k_{\perp}^{2}} 4 C_{F} \frac{\alpha_{s}\left(k_{\perp}^{2}\right)}{4 \pi}\right] \frac{d k}{k}=\text { const } \cdot \frac{d k}{k} .
$$

Notice that what we got is nothing but the famous hadronic plateau of the old parton picture. It appears due to sequential acts of blanching color fields in the spreading $q \bar{q}$ system. 
Blanching Secondary Partons. How will additional PT gluons contribute to the hadron yield? We met already two characteristic time scales (1.7) and (1.8), namely formation and hadronization times of a secondary gluon. Now let us introduce one more important point $t^{\text {separ }}$ when the separation in the transverse plane $\Delta \rho_{\perp} \approx t \cdot c \Theta$ between the bremsstrahlung particle (with emission angle $\Theta$ ) and its parent will reach critical confinement distance $R$. Note that our three time scales characterizing the gluon's being are parametrically ordered with the PT parameter (1.12):

$$
\begin{aligned}
t^{\text {form }} & \approx \frac{k}{k_{\perp}{ }^{2}}=t^{\text {form }} * 1, \\
t^{\text {separ }} & \approx \frac{R}{\Theta}=t^{\text {form }} *\left(k_{\perp} R\right), \\
t^{\text {hadr }} & \approx k R^{2}=t^{\text {form }} *\left(k_{\perp} R\right)^{2} .
\end{aligned}
$$

At the same time all three are of the same order for gluers.

At $t^{\text {separ }}$ some new specific non-PT interaction must take place to screen the total color charge of the outgoing gluon (e.g., with a help of light $q \bar{q}$ pairs created from the vacuum in an octet state). Our qualitative estimates do support this need. Exactly in time appropriate gluers are formed which follow the gluon:

$$
\begin{gathered}
\Theta_{\text {gluer }} \approx \Theta, \quad\left(k_{\perp}\right)_{\text {gluer }} \sim R^{-1}, \quad \Longrightarrow \quad k_{\text {gluer }} \approx \frac{1}{R \Theta} ; \\
\left(t^{\text {form }} \sim t^{\text {separ }} \sim t^{\text {hadr }}\right)_{\text {gluer }} \approx \frac{R}{\Theta}=\left(t^{\text {separ }}\right)_{q g} .
\end{gathered}
$$

Starting from $t=t^{\text {separ }}$ the gluon becomes an independent source of hadrons with energies from $k_{\text {gluer }}$ and up to $k$. This additional plateau, from the PT point of view, should be roughly twice as large as the quark induced spectrum 
(1.13) since the gluon radiates gluons (and gluers?) $N_{c} / C_{F}=9 / 4$ times more intensely than the quark does. It looks like a jet produced in an interaction with effective hardness $Q^{2} \sim k_{\perp}^{2}$ but boosted with the Lorentz-factor $\gamma=1 / \Theta$.

At first glance it might seem strange that the new subjet did not contribute to the yield of softest hadrons:

$$
R^{-1} \lesssim \omega_{\text {hadr }} \lesssim R^{-1} / \Theta .
$$

This is, in fact, an interesting phenomenon which stems from the very nature of QCD as a gauge theory. The reason for that is the conservation of color current: soft hadrons with energies in the interval (1.14) are formed too early $\left(t<t^{\text {separ }}\right)$ when $q$ and $g$ appear to be so close to each other that they act with respect to gluers as a single emitter with total color charge equal to that of the original quark.

If the hard gluon of Fig. 1.2 contributed to the hadron yield with a "full" plateau built up of hadrons with energies

$$
R^{-1} \lesssim \omega_{h a d r} \lesssim k,
$$

one would expect ${ }^{2}$ the final spectrum resulting from the multiple cascade parton emissions to be strongly populated with softest hadrons: $\omega_{\text {hadr }} \sim m_{\text {hadr }}$. This common wisdom however was proved to be wrong in the beginning of eighties when the Quantum-Mechanical Coherence was rediscovered in the QCD context.

\footnotetext{
${ }^{2}$ and this is inherent to so called Independent Fragmentation schemes of hadronization modelling
} 


\subsection{Partons in Field Theory}

Despite the smallness of the effective coupling at small space-time intervals, the PT description of hard processes does not result, in general, in a trivial power series over $\alpha_{s}$. The partonic structure of hard processes, whether one deals with the content of jets produced in $e^{+} e^{-}$annihilation or with spacetime fluctuations caught by a snapshot of DIS, appears to be rather rich.

Studying various characteristics of strong interactions at high energies, we face, in one way or another, consequences of the fact that specific QCD parton multiplication processes happen to have large probabilities. We have seen this already considering typical process of gluon bremsstrahlung, (1.6), where the total probability of a gluon emission turned out to be large due to broad logarithmic integrations over $k$ and $k_{\perp}^{2}$.

Multiple QCD bremsstrahlung demandes a basic new approach for the description of the structure of partonic systems determining the basic properties of hard processes of lepton-hadron and hadron-hadron interactions.

\subsubsection{Leading Logarithmic Approximation}

Historically this approach was first tried in attempts to reach a profound level of understanding Bjorken scaling in lepton-hadron scattering with large momentum transfer

$$
Q^{2}=-q^{2} \gg M^{2} \quad, \quad \nu=\frac{(p \cdot q)}{M} \gg M .
$$

Approximate Bjorken scaling has been found to hold in Quantum Field Theory in the pioneering works by Gribov and Lipatov of 1972 . It was shown that in a field theory with dimensionless coupling constant $g$ DIS structure functions can be represented as a sum of Rutherford cross sections of the lepton scattering off a pointlike charged particle weighted with parton densities 
$D_{i}^{f}(x)$, where $x$ is the fraction of the longitudinal momentum $p$ of the fast projectile $(i)$ carried by the parton $(f)$.

Instead of fixed, $Q^{2}$-independent, parton distributions which would correspond to exact scaling, logarithmic deviations from the true scaling behavior had been predicted, revealing the internal structure of parton densities $D_{i}^{f}\left(x, \ln Q^{2}\right)$. The $Q^{2}$-dependence comes from summing the leading logarithmic corrections and physically represents the fact that a deep inelastic scattering involving a photon of virtuality $Q^{2}$ corresponds to the scattering of that virtual photon off a quark of transverse size $1 / Q$.

The point is that the large momentum transfer $Q^{2}$ sets an upper bound for transverse momenta of partons logarithmically distributed over the broad range

$$
d w \propto g^{2} \int^{Q^{2}} \frac{d k_{\perp}^{2}}{k_{\perp}^{2}}
$$

which makes the total probability of extra parton production large:

$$
w \propto g^{2} \cdot \ln Q^{2} \sim 1 \quad ; \quad g^{2} \ll 1,
$$

in spite of the smallness of the field coupling $g^{2}$ which is required for the perturbative calculations to be applicable. This observation formed the base of the so called Leading Logarithmic Approximation (hereafter LLA).

In the LLA framework one keeps trace of contributions to structure functions of the order

$$
W\left(x, Q^{2}\right) \propto D_{i}^{f}\left(x, \ln Q^{2}\right)=\sum_{n=0}^{\infty} f_{n}(x)\left(\frac{\alpha_{s}}{\pi} \ln Q^{2}\right)^{n}
$$

while neglecting systematically corrections of the relative order $\alpha_{s}$, not accompanied by a large log. In a field theory with asymptotic freedom such an 
approximation proves to be asymptotically exact, i.e.

$$
W^{\text {true }}\left(x, Q^{2}\right)=W^{L L A}\left(x, Q^{2}\right)\left[1+O\left(\frac{\alpha_{s}\left(Q^{2}\right)}{\pi}\right)\right] \stackrel{Q^{2} \rightarrow \infty}{\rightarrow} W^{L L A}\left(x, Q^{2}\right) .
$$

Gribov and Lipatov have analyzed Feynman diagrams contributing to the discontinuity of the forward lepton- "parton" scattering amplitudes determining DIS structure functions in field theories wherein fermions are coupled with pseudoscalar and Abelian vector bosons:

$$
L_{\text {int }}=\bar{\psi} \gamma_{5} \psi \cdot \phi \quad, \quad L_{i n t}=\bar{\psi} \gamma_{\mu} \psi \cdot A^{\mu} .
$$

The same LLA program was realized also for inclusive $x$-distributions of "partons" produced in $e^{+} e^{-}$annihilation.

It is important to emphasize, that in spite of rather complicated character of the original Feynman diagrams contributing to LLA, the final answer proved to be very transparent, allowing a simple probabilistic interpretation of basic processes that determine the internal structure of partonic systems and resulting parton densities.

Explicit partonic interpretation of the structure of DIS in logarithmic field theories was given in Refs. [56] where the probabilistic Evolution Equations determining the $Q^{2}$ dependence of parton densities $D_{i}^{f}(x)$ have been interpreted in terms of the kinetic equilibrium of partons inside a hadron under the variation of the ultraviolet transverse momentum cut-off $\Lambda=Q$.

The LLA parton wavefunction of a projectile can be built up iterating elementary parton splitting processes $1 \rightarrow 2$ with successively increasing transverse momenta

$$
\mu^{2} \ll k_{\perp_{1}}^{2} \ll k_{\perp_{2}}^{2} \ll \cdots \ll k_{\perp_{n-1}}^{2} \ll k_{\perp_{n}}^{2} \ll Q^{2} .
$$

Formally the strong transverse momentum ordering (1.16) is necessary to gain the maximal logarithmic contribution to (1.15) in the $n^{\text {th }}$ PT-order. 
Physically this means that partons of the (logarithmic) field theory are not point-like particles: an attempt to localize the parton of the $i^{\text {th }}$ generation, which has typical transverse size $\Delta \rho_{\perp}^{(i)} \sim 1 / k_{\perp i}$, reveals its substructure at smaller distances $\Delta \rho_{\perp} \sim 1 / k_{\perp i+1} \ll \Delta \rho_{\perp}^{(i)}$. With respect to the small-distance probe our parton behaves basically as a real particle in the spirit of the wellknown Weitzsäcker-Williams method of equivalent photons in QED, which has been generalized in Ref. [7] to embedd quasireal electrons and successfully applied for derivation of the QCD parton evolution picture by Altarelli and Parisi 8]. The Feynman diagram LLA-technique was implemented for QCD case in Ref. [9].

The second approach invented by Christ, Hasslacher and Mueller 10] was based on the Wilson operator product expansion (OPE) and exploited the renormalization group (RG) properties of scattering amplitudes. The moments of the DIS structure functions are factorized here in two parts: matrix elements of local operators and their coefficient functions which appear in the light-cone expansion for the $T$-product of two electromagnetic currents. For large $Q^{2}$, the dominant contribution comes from the operators of minimal twist $t=d-m=2$ with $d$ the canonical dimension of the operator and $m$ its Lorentz spin. Higher twist operators with $t=4,6 \ldots$ give power suppressed contributions $\sim Q^{2-t}$ which may be significant at moderately high $Q^{2}$.

The RG technique was developed for QCD applications by Gross \& Wilczek and Georgi \& Politzer 11.

Combined with the operator product expansion a satisfying picture emerged. One can express the structure functions either in terms of the parton model where the leptons scatter off the quark constituents of the hadronic target, say a proton, or in terms of a bilocal product of quark operators. Thus 
in the parton model

$$
\frac{1}{M} \nu W_{2}\left(x, Q^{2}\right)=\sum_{f} e_{f}^{2}\left(x q_{f}\left(x, Q^{2}\right)+x \bar{q}_{f}\left(x, Q^{2}\right)\right)
$$

where $q_{f}\left(\bar{q}_{f}\right)$ gives the number density of quarks (antiquarks) of flavor $f$ and having a fraction $x$ of the proton's momentum in a frame where the proton's momentum is large. $e_{f}$ is the electric charge of the quark of flavor $f$, in units of the electron charge. In terms of the OPE the $m^{\text {th }}$ moment of $q\left(x, Q^{2}\right)+\bar{q}\left(x, Q^{2}\right)$ is given by

$$
\int_{0}^{1} d x\left[q_{f}\left(x, Q^{2}\right)+\bar{q}_{f}\left(x, Q^{2}\right)\right] x^{m}=\frac{1}{2 p_{+}^{m+1}}\left\langle p\left|\tilde{q}_{f} \gamma_{+}\left(\frac{i \overleftrightarrow{D_{+}}}{2}\right)^{m} q_{f}\right| p\right\rangle
$$

where $D_{\mu}$ is the covariant derivative, and the renormalization of the quark operator whose proton matrix element is on the right hand side of (1.18) is at a scale $Q^{2}$. Thus matrix elements of gauge invariant local quark operators are related to moments of the parton distributions in the QCD improved (LLA) parton model which in turn are related to the measured data by (1.17). An entirely satisfactory situation.

However, the parton model interpretation of the OPE can be justified only at the twist-two level. Beyond the leading twist more general quantities parton correlation functions - emerge, which are governed by the multiparticle Faddeev type evolution equations with pairwise particle interaction 12. In the next Chapter we shall discuss in detail the very concept of partons in connection with a subtlety in the partonic interpretation which appears in spin dependent DIS. In this Chapter we restrict ourselves to the Basics of LLA which can be learned with the help of many review papers (see, e.g., Refs. [13,15]). Here we give a brief overview of the main ingredients of the subject which will be of use in what follows. They are: 
- basic structure of the "parton ladder",

- parton splitting functions,

- running coupling in parton cascades and

- double logarithmic parton form factors.

\subsubsection{Notations and Kinematics}

Recall the relationship between the cross section for deep inelastic polarized electron (or muon) scattering off a polarized nucleon 16 as illustrated in Fig. [1.3.

$$
d \sigma=\frac{\alpha^{2}}{\pi} \frac{1}{Q^{4}} L_{\mu \lambda}(\zeta) W_{\mu \lambda}(s) \frac{d^{3} k^{\prime}}{(p \cdot k) E^{\prime}}
$$

where $q^{2}=-Q^{2}$ with $q$ the momentum transfer from the leptons to the nucleon, $p$. The lepton tensor $L_{\mu \lambda}$ is given by

$$
L_{\mu \lambda}(\zeta)=\sum_{\zeta^{\prime}}\left[\tilde{u}\left(k^{\prime}, \zeta^{\prime}\right) \gamma_{\mu} u(k, \zeta)\right]^{*}\left[\tilde{u}\left(k^{\prime}, \zeta^{\prime}\right) \gamma_{\lambda} u(k, \zeta)\right]
$$

where the spin vector, $\zeta_{\mu}$, of the initially polarized electron is determined by (with $\vec{\zeta}$ a unit vector)

$$
\zeta_{\mu}=\left(\frac{\vec{k} \cdot \vec{\zeta}}{m}, \vec{\zeta}+\frac{\vec{k}(\vec{k} \cdot \vec{\zeta})}{m(E+m)}\right) .
$$

For example an electron having momentum $k$ and helicity +1 has a corresponding $\vec{\zeta}$ vector given by $\vec{\zeta}=\vec{k} /|\vec{k}|$. The sum over $\zeta^{\prime}$ in (1.20) is to be 


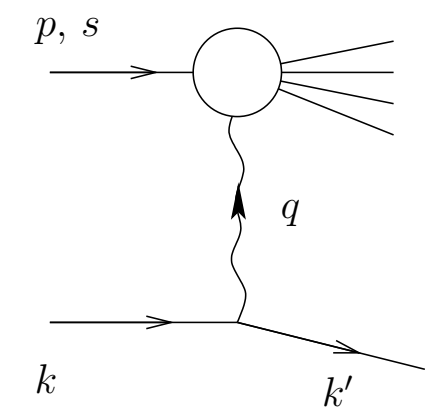

Figure 1.3: Kinematics of deep inelastic lepton-hadron scattering.

taken over the two independent spins of the final electron of momentum $k^{\prime}$. From (1.20) one finds

$$
L_{\mu \lambda}(\zeta)=2\left(k_{\mu} k_{\lambda}^{\prime}+k_{\lambda} k_{\mu}^{\prime}-\left(k \cdot k^{\prime}\right) g_{\mu \lambda}\right)-2 i m \epsilon_{\mu \lambda \rho \sigma} q_{\rho} \zeta_{\sigma}
$$

in the approximation of neglecting $m^{2}$ compared to $k \cdot k^{\prime}$. The hadronic tensor $W_{\mu \lambda}$ is given by

$$
W_{\mu \lambda}(s)=\frac{1}{4} \sum_{n}\left\langle p s\left|J_{\mu}(0)\right| n\right\rangle\left\langle n\left|J_{\lambda}(0)\right| p s\right\rangle(2 \pi)^{4} \delta^{4}\left(q+p-p_{n}\right)
$$

with the four-vector $s_{\mu}$ specifying the spin of the nucleon. $J_{\mu}(x)$ is the electromagnetic current. In our normalization $\tilde{u}(p, s) u(p, s)=2 M$ and $\tilde{u}(p, s) \gamma_{\mu} \gamma_{5} u(p, s)=2 M s_{\mu}$ with $s_{\mu}$ defined for nucleons exactly as $\zeta_{\mu}$ was for leptons. Due to conservation of the electromagnetic current, tensor $W_{\mu \lambda}(s)$ is orthogonal to the photon momentum $q_{\mu}\left(q_{\lambda}\right)$ and has the structure function decomposition

$$
W_{\mu \lambda}(s)=W_{\mu \lambda}+i W_{\mu \lambda}^{a}
$$


with

$$
\frac{1}{\pi} W_{\mu \lambda}=-\left(g_{\mu \lambda}-\frac{q_{\mu} q_{\lambda}}{q^{2}}\right) W_{1}+\frac{1}{M^{2}}\left(p_{\mu}-\frac{p \cdot q}{q^{2}} q_{\mu}\right)\left(p_{\lambda}-\frac{p \cdot q}{q^{2}} q_{\lambda}\right) W_{2}
$$

and

$$
\frac{1}{\pi} W_{\mu \lambda}^{a}=\frac{1}{M} \epsilon_{\mu \lambda \rho \sigma} q_{\rho}\left[s_{\sigma}\left(G_{1}+\frac{p \cdot q}{M^{2}} G_{2}\right)-p_{\sigma} \frac{s \cdot q}{M^{2}} G_{2}\right] .
$$

The structure functions $W_{1}, W_{2}, G_{1}$ and $G_{2}$ are functions of the two independent invariants $\nu=(p \cdot q) / M$ and $Q^{2}$ or, equivalently, of the variables $x=Q^{2} / 2(p \cdot q)$ and $Q^{2}$.

$W_{1}$ and $W_{2}$ are measured in spin independent DIS according to

$$
\frac{d \sigma}{d \Omega d E^{\prime}}=\frac{4 \alpha^{2} E^{\prime 2}}{Q^{4} M}\left[W_{2} \cos ^{2} \frac{\Theta}{2}+2 W_{1} \sin ^{2} \frac{\Theta}{2}\right]
$$

with $\Theta$ the angle of scattering between the final and initial leptons in the nucleon rest system.

Analogously in spin dependent lepton nucleon scattering

$$
\frac{d \sigma^{\uparrow \downarrow}}{d \Omega d E^{\prime}}-\frac{d \sigma^{\uparrow \uparrow}}{d \Omega d E^{\prime}}=\frac{4 \alpha^{2} E^{\prime}}{Q^{2} M^{3} E}\left[M G_{1}\left(E+E^{\prime} \cos \Theta\right)-Q^{2} G_{2}\right]
$$

where $(\uparrow \uparrow)$ refers to initial electron and nucleon spins parallel to the initial electron momentum $\vec{k}$ while $(\uparrow \downarrow)$ refers to the initial electron spin parallel to its direction of motion while the target nucleon spin is opposite to the direction of $\vec{k}$. There are also expressions for transversely polarized electrons which will not concern us here.

The structure functions are related to scaling functions according to

$$
\begin{aligned}
W_{1} & =F_{1}, & & \frac{1}{M} \nu W_{2}=F_{2} ; \\
\left(\frac{\nu}{M}\right) G_{1} & =g_{1}, & & \left(\frac{\nu}{M}\right)^{2} G_{2}=g_{2}
\end{aligned}
$$


where $F_{1}, F_{2}, g_{1}, g_{2}$ have only logarithmic dependence on $Q^{2}$ when they are considered as functions of $x$ and $Q^{2}$.

Referring to (1.27) we see that a DIS measurement using longitudinally polarized electrons or muons is capable of giving a determination of $g_{1}$ but a determination of $g_{2}$ is difficult because $g_{2}$ appears in (1.27) multiplied by an additional power of $1 / Q^{2}$ as compared to $g_{1}$. We shall return to the experimental situation later on in the next Chapter.

\subsubsection{Parton Ladder Building}

DIS cross section can be obtained via the discontinuity of the amplitude of forward virtual photon scattering off the nucleon. In the case of an unpolarized target there are only two structures (1.24) connected with inelastic "form factors" $W_{1}$ and $W_{2}$ of the target particle that contribute.

\section{Sudakov variables}

In hard interactions, in general, symmetry among the 3 space directions is strongly broken. In DIS, where a large momentum $q$ is transferred from the scattered lepton to "heat" the hadron, it is the direction of the "colliding" virtual photon and nucleon which induces such anisotropy. It proves to be very useful to exploit this fact and represent all particle momenta in terms of the Sudakov decomposition:

$$
\begin{aligned}
k_{\mu} & =\alpha \cdot q_{\mu}^{\prime}+\beta \cdot p_{\mu}^{\prime}+k_{\perp \mu} \\
\left(k_{\mu}\right)^{2} & =\alpha \cdot \beta \cdot s-k_{\perp}^{2} \quad\left(k_{\perp}^{2} \equiv{\overrightarrow{k_{\perp}}}^{2}>0\right) \\
d^{4} k & =\frac{s}{2} d \alpha d \beta d^{2} \overrightarrow{k_{\perp}}
\end{aligned}
$$


where $q^{\prime}$ and $p^{\prime}$ are the light-like vectors defining the interaction hyperplane:

$$
\begin{array}{cl}
q_{\mu}=q_{\mu}^{\prime}-x \cdot p_{\mu}^{\prime}, & p_{\mu}=p_{\mu}^{\prime}+\frac{M^{2}}{s} \cdot q_{\mu}^{\prime} \approx p_{\mu}, \\
p^{\prime 2}=q^{\prime 2}=0 \quad, & s \equiv 2\left(p^{\prime} \cdot q^{\prime}\right) \approx 2(p \cdot q) .
\end{array}
$$

Hereafter we shall neglect effects of a finite proton mass $M^{2} \ll Q^{2}$ identifying $p_{\mu}^{\prime} \approx p_{\mu}$.

\section{Born approximation}

Imagine that it is some point-like fermion with momentum $p$ which takes an impact. In this case the discontinuity of the forward scattering amplitude averaged over initial parton polarizations reads simply

$$
\frac{1}{\pi} W_{\mu \lambda}=e^{2} \delta\left((p+q)^{2}\right) \frac{1}{2} \operatorname{Tr}\left\{\hat{p} \gamma_{\mu}(\hat{p}+\hat{q}) \gamma_{\lambda}\right\} .
$$

Invoking (1.30) we have

$$
(p+q)=(0+1) q^{\prime}+(1-x) p+(\overrightarrow{0}+\overrightarrow{0})_{\perp},(p+q)^{2}=(1-x) s, \delta\left((p+q)^{2}\right)=\frac{1}{s} \delta(1-x)
$$

and the Born amplitude takes the form

$$
\frac{1}{\pi} W_{\mu \lambda}^{(0)}(x)=e^{2} \delta(1-x) \frac{1}{2 s} \operatorname{Tr}\left\{\hat{p} \gamma_{\mu} \hat{q}^{\prime} \gamma_{\lambda}\right\}=e^{2} \delta(1-x)\left(-g_{\mu \lambda}^{\perp}\right)
$$

with $g^{\perp}$ the 4 -tensor orthogonal to the scattering hyperplane:

$$
g_{\mu \lambda}^{\perp}=g_{\mu \lambda}-\frac{p_{\mu} q_{\lambda}^{\prime}+q_{\mu}^{\prime} p_{\lambda}}{\left(p \cdot q^{\prime}\right)} .
$$


This means that only transversally polarized photons can be absorbed by a fermion-parton. Comparing (1.24), (1.28a) to (1.32) one obtains immediately

$$
\frac{1}{x} F_{1}=F_{2}=e^{2} \delta(1-x)
$$

\section{One-loop approximation}

$x=1$ corresponds to elastic photon-parton interaction. Now let us turn on parton interactions to open the inelastic channel. The simplest first order diagram is shown in Fig. 1.4. To start with, let us choose for simplicity the wavy line in Fig. 1.4 to stand for a scalar boson ("gluon") mediating the interaction between fermions ("quarks").
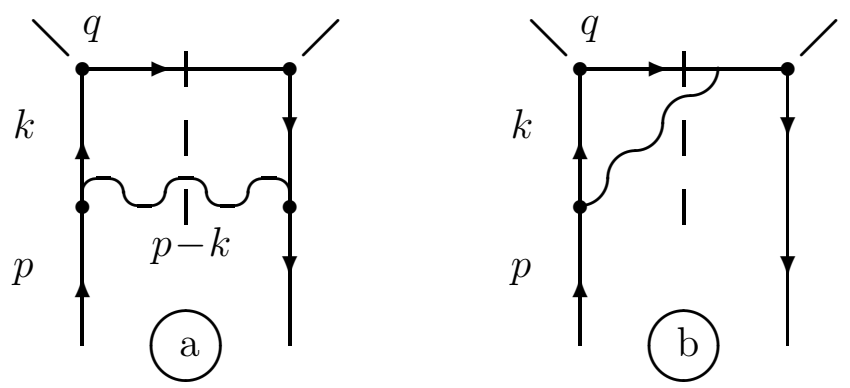

Figure 1.4: First order ladder (a) and interference (b) diagrams for inelastic lepton-parton scattering.

Then the imaginary part of the forward scattering amplitude will read

$$
\frac{1}{\pi} W_{\mu \lambda}=e^{2} g^{2} \int \frac{d^{4} k}{(2 \pi)^{4}} \delta\left((k+q)^{2}\right) \frac{1}{2} \operatorname{Tr}\left[\hat{p} \hat{k} \gamma_{\mu}(\hat{k}+\hat{q}) \gamma_{\lambda} \hat{k}\right] \frac{1}{k^{4}}(2 \pi) \delta\left((p-k)^{2}\right)
$$


with $g$ the "quark"-"gluon" coupling. Now we have to integrate over virtual quark momentum $k_{\mu}$. In terms of Sudakov variables (1.29) $\delta$-functions take the form

$$
\begin{gathered}
\delta\left((k+q)^{2}\right) \delta\left((p-k)^{2}\right)=\delta\left((1+\alpha)(\beta-x) s-k_{\perp}^{2}\right) \delta\left(-\alpha(1-\beta) s-k_{\perp}^{2}\right) \\
=\frac{1}{s^{2}} \frac{1}{(1-\beta)-k_{\perp}^{2} / s} \delta\left(\beta-x-\frac{k_{\perp}^{2}}{1-k_{\perp}^{2} /(1-\beta) s}\right) \delta\left(-\alpha-\frac{k_{\perp}^{2}}{(1-\beta) s}\right)
\end{gathered}
$$

and can be used to take away integrations over the $\alpha$ and $\beta$ components of $k$. Noticing that the dominant contribution to (1.51) comes from kinematical region

$$
\beta \approx x \sim 1 \quad, \quad-\alpha \sim k_{\perp}^{2} / s \ll 1
$$

we may extract the zero-order structure (1.32) to obtain (see Problem 1.1)

$$
\frac{1}{\pi} W_{\mu \lambda}^{(1)}=\int \frac{d k_{\perp}^{2}}{k_{\perp}^{2}} \frac{g^{2}}{16 \pi^{2}} \int_{0}^{1} \frac{d \beta}{\beta} \cdot(1-\beta) \cdot\left[\frac{1}{\pi} W_{\mu \lambda}^{(0)}\left(\frac{x}{\beta}\right)\right]
$$

The main lesson we should deduce from this exercise is the large contribution arising from logarithmically distributed transverse momenta of the parton which takes the impact. In the LLA kinematics (1.36) its momentum $k$ can be said to be quasi-collinear to the direction of the incoming particle: $k_{\mu} \approx \beta \cdot p_{\mu}$. Moreover, with respect to the hard photon-parton interaction $k$ behaves as quasi-real, since one is forced to neglect its finite virtuality

$$
-k^{2}=-\alpha \beta s+k_{\perp}^{2}=\frac{k_{\perp}^{2}}{(1-\beta) s} \beta s+k_{\perp}^{2}=\frac{k_{\perp}^{2}}{1-\beta} \ll Q^{2}
$$

in the upper part of the amplitude of Fig. 1.4 to keep the large log. 
Operationally, the origin of the collinear log is quite simple: extra particle emission has added two quasi-real propagators which had been only partially compensated by the numerator of the Feynman amplitude:

$$
W \propto d k_{\perp}^{2} \frac{1}{\left(k^{2}\right)^{2}} \operatorname{Tr}\{\hat{p} \hat{k} \ldots \hat{k}\} \sim d k_{\perp}^{2} \frac{1}{\left(k_{\perp}^{2}\right)^{2}} k_{\perp}^{2} \operatorname{Tr}\{\hat{p} \ldots\}
$$

Now it becomes clear that the non-ladder diagrams in our toy model with scalar gluons will not contribute to the LLA. Indeed, for the interference diagram of Fig. 1.4b, e.g., one has the estimate

$$
W \propto d k_{\perp}^{2} \frac{1}{\left(k^{2}\right)} \operatorname{Tr}\{\hat{p} \ldots \hat{k}\} \sim d k_{\perp}^{2} \frac{1}{\left(k_{\perp}^{2}\right)}\left\{\hat{p} \ldots \hat{k}_{\perp}\right\}
$$

which is no longer logarithimic in $k_{\perp}$.

\section{Multi-parton ladders}

Following the same logic, we can build up the ladder of successive parton decays shown in Fig. 1.57. Generalization of the lowest order calculation is straightforward. In order to obtain a large log from the second cell one has to restrict the region of integration over $k_{\perp_{2}}$ by inequality $k_{\perp_{2}}^{2} \gg k_{\perp_{1}}^{2}$, and so on. Integrations over $q^{\prime}$-components of "gluon" momenta $\alpha_{i}$ one performs using the $\delta$-functions of ladder rungs

$$
\begin{array}{r}
\delta\left(\left(k_{i}-k_{i-1}\right)^{2}\right)=\delta\left(\left(\alpha_{i}-\alpha_{i-1}\right)\left(\beta_{i}-\beta_{i-1}\right) s-\left({\overrightarrow{k_{\perp i}}}_{-k_{\perp i-1}}\right)^{2}\right) \\
-\alpha_{i-1} \ll-\alpha_{i} \approx \frac{k_{\perp i}^{2}}{s}\left(\beta_{i-1}-\beta_{i}\right) \ll 1, \quad \beta_{i} \sim 1
\end{array}
$$

in the region of strongly ordered transverse momenta of emitted "gluons" according to (1.16). 

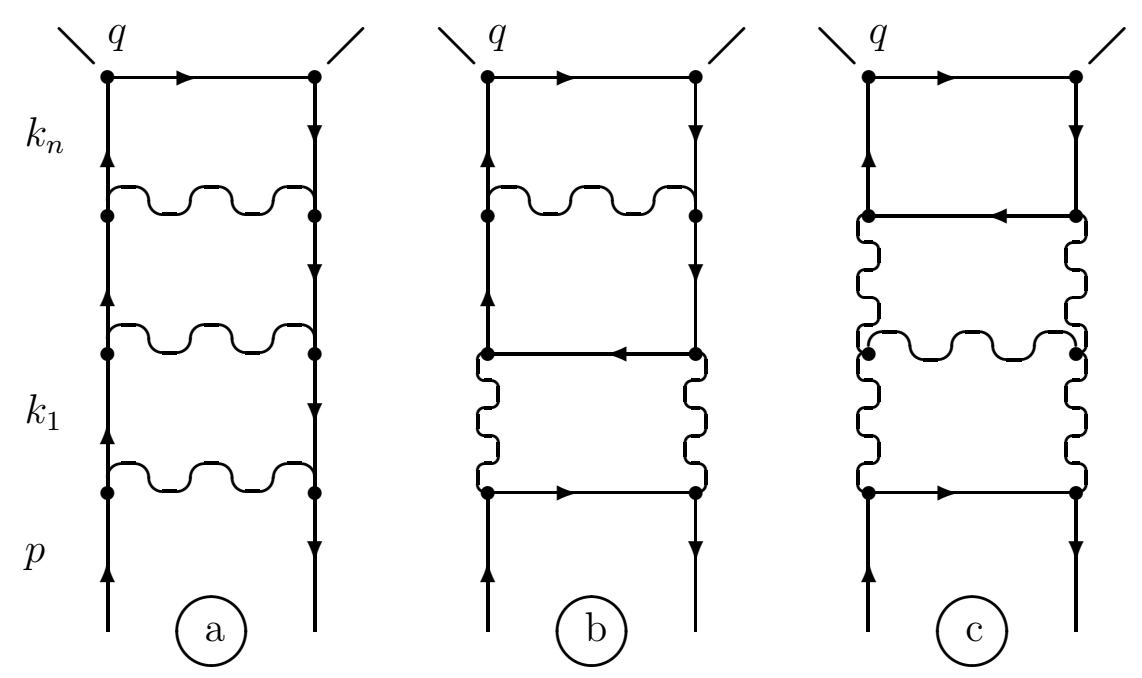

Figure 1.5: Examples of multi-cell LLA ladder diagrams: valence quark propagation (a), sea-quark-gluon mixing (b), with gluon self-interactions (c).

This results in the $n^{\text {th }}$ order contribution to the LLA:

$$
\left(\frac{g^{2}}{16 \pi^{2}}\right)^{n} \int_{\mu^{2}}^{Q^{2}} \frac{d k_{\perp_{n}}^{2}}{k_{\perp n}^{2}} \int_{\mu^{2}}^{k_{\perp n}^{2}} \frac{d k_{\perp_{n-1}}^{2}}{k_{\perp n-1}^{2}} \ldots \int_{\mu^{2}}^{k_{\perp 2}^{2}} \frac{d k_{\perp 1}^{2}}{k_{\perp 1}^{2}}=\frac{1}{n !}\left(\frac{g^{2}}{16 \pi^{2}} \ln \frac{Q^{2}}{\mu^{2}}\right)^{n}
$$

$\beta$-components of fermion momenta decrease when one moves "up" along the ladder:

$$
1 \geq \beta_{1} \geq \beta_{2} \geq \ldots \geq \beta_{n-1} \geq \beta_{n} \geq x
$$


Corresponding $x$-dependence will be given then by the convolution

$$
\int_{0}^{1} \frac{d \beta_{n}}{\beta_{n}} \delta\left(1-\frac{x}{\beta_{n}}\right) \int_{\beta_{n}}^{1} \frac{d \beta_{n-1}}{\beta_{n-1}} \ldots \int_{\beta_{2}}^{1} \frac{d \beta_{1}}{\beta_{1}} \Phi_{F}^{F}\left(\frac{\beta_{n}}{\beta_{n-1}}\right) \Phi_{F}^{F}\left(\frac{\beta_{n-1}}{\beta_{n-2}}\right) \ldots \Phi_{F}^{F}\left(\frac{\beta_{n}}{1}\right)
$$

where $\Phi_{F}^{F}(z)=(1-z)$ is the $\beta$-kernel of (1.37) describing the fermion-tofermion transition with momentum fraction $z$ carried by the offspring parton with larger transverse momentum (virtuality). It is important to emphasize, that this kernel does not depend on the location of the decay cell in the ladder.

Similar LLA contributions arise from ladders of Fig. 1.5a,b where fermionic and bosonic states mix with each other in $t$-channel.

\section{Vector "gluons"}

The LLA analysis appears to be slightly more complicated for the case of vector bosons as "gluons". The expression for a single ladder cell of Fig. [1.4a now reads

$$
W_{\mu \lambda}=e^{2} g^{2} \int \frac{d^{4} k}{(2 \pi)^{4}} \delta\left((k+q)^{2}\right) \frac{1}{2} \operatorname{Tr}\left\{\hat{p} \gamma_{\rho} \hat{k} \gamma_{\mu}(\hat{k}+\hat{q}) \gamma_{\lambda} \hat{k} \gamma_{\sigma}\right\} \frac{-g_{\rho \sigma}}{k^{4}} 2 \pi \delta\left((p-k)^{2}\right)
$$

where we used the Feynman gauge polarization tensor $g_{\rho \sigma}$ to sum up over "gluon" spin states. The following chain of transformations

$$
\operatorname{Tr}\left\{\hat{p} \gamma_{\rho} \hat{k} \ldots \hat{k} \gamma_{\sigma}\right\} \cdot\left(-g_{\rho \sigma}\right)=2 \operatorname{Tr}\{\hat{p} \hat{k} \ldots \hat{k}\} \approx 2 k_{\perp}^{2} \operatorname{Tr}\{\hat{p} \ldots\}
$$

leads to the kernel similar to that for the scalar case (1.37):

$$
\left\{\Phi_{F}^{F}(z)\right\}_{\text {ladder }}=2(1-z)
$$


This is however not the whole story. Indeed, coming back to non-ladder amplitude of Fig. 1.4b, one obtains now the logarithmic contribution with only one quasi-real fermion propagator:

$$
W \propto d k_{\perp}^{2} \frac{1}{\left(k^{2}\right)} \operatorname{Tr}\left\{\hat{p} \ldots \hat{k} \gamma_{\mu}\right\} \sim d k_{\perp}^{2} \frac{1}{\left(k_{\perp}^{2}\right)}\{\hat{p} \ldots\} \cdot 2 \beta p_{\mu} .
$$

Thus, because of vector bosons with polarizations in the scattering plan 3 , locality of parton decays in the LLA is lost and the general high order analysis seems to be a mess. However, these interference contributions can be effectively summed up 4 .

The key idea is to replace, within the LLA accuracy, the vertex factor $p_{\mu}$ in (1.47) by the "gluon" momentum

$$
p_{\mu}=\frac{1}{1-\beta}(p-k)_{\mu}+O\left(k_{\perp}\right)
$$

and then to make use of the conservation of current to reduce the sum of interference diagrams (with the line $p-k$ attached to any place above the emission cell) to the local integral 4

$$
\frac{1}{\pi} W_{\mu \lambda}=\int \frac{d k_{\perp}^{2}}{k_{\perp}^{2}} \frac{g^{2}}{16 \pi^{2}} \int_{0}^{1} \frac{d \beta}{\beta} \cdot \frac{2 \beta}{1-\beta} \cdot\left[\frac{1}{\pi} W_{\mu \lambda}^{(U P)}\left(\frac{x}{\beta}\right)\right]
$$

where we denoted by $W^{(U P)}$ the "upper block" of the scattering amplitude. Equation (1.48) gives rise to the second part of the parton splitting kernel $\Phi$ which finally takes the form

$$
\Phi_{F}^{F}(z)=\left\{\Phi_{F}^{F}(z)\right\}_{\text {ladder }}+2 \cdot\left\{\Phi_{F}^{F}(z)\right\}_{\text {interf. }}=2(1-z)+2 \cdot \frac{2 z}{(1-z)}=2 \frac{1+z^{2}}{1-z}
$$

\footnotetext{
${ }^{3}$ notice that the "ladder gluons" in (1.45) had effectively only transverse polarizations
} 
where we took into account an additional factor 2 for the number of interference graphs.

For the real quark-gluon interaction the splitting function (1.49) has to be supplied with the color factor $C_{F}$ originating from summation (averaging) over color states of final (initial) partons:

$$
\frac{1}{N_{c}} \sum_{a} \operatorname{Tr}\left(t^{a} \cdot t^{a}\right)=\frac{N_{c}^{2}-1}{2 N_{c}} \equiv C_{F} .
$$

\section{"Physical" gauges}

Instead of dealing separately with transversally and longitudinally polarized bosons one can get the same result by a special choice of the gauge used to describe vector fields. The most physically transparent way to build up the LLA is provided by so called physical gauges, where only ladder diagrams contribute as it was in the case of scalar bosons considered above. The axial gauge is an example of such a choice 5 , when one takes the vector potential to be orthogonal to $q^{\prime}$ :

$$
A_{\mu} \cdot q_{\mu}^{\prime}=0
$$

The propagator of the vector field with momentum $k$ now reads

$$
\begin{aligned}
D_{\mu \lambda} & =\frac{d_{\mu \lambda}}{k^{2}+i \epsilon} \\
d_{\mu \lambda} & =g_{\mu \lambda}-\frac{k_{\mu} q_{\lambda}^{\prime}+k_{\lambda} q_{\mu}^{\prime}}{\left(k \cdot q^{\prime}\right)} .
\end{aligned}
$$

As compared to the Feynman gauge with $d_{\mu \lambda}=g_{\mu \lambda}$, $\operatorname{Tr} g_{\mu \lambda}=4$, now only 2 physical polarizations propagate on mass shell:

$$
d_{\mu \mu}(k)=2 ; \quad k_{\mu} d_{\mu \nu}(k)=-k^{2} \frac{q_{\nu}^{\prime}}{\left(k q^{\prime}\right)}=0 \quad \text { at } \quad k^{2}=0 .
$$


Notice, that in the quasi-collinear limit $k_{\mu} \approx \beta p_{\mu}$ the polarization tensor (1.51) becomes simply

$$
d_{\mu \nu}(k)=g_{\mu \nu}^{\perp}+O\left(k_{\perp}\right)
$$

Now the non-ladder amplitudes of the type of Fig. 1.40 will no longer contribute to the LLA, since the corresponding structure (1.47) proves to be suppressed:

$$
\begin{gathered}
W \propto d k_{\perp}^{2} \frac{1}{\left(k_{\perp}^{2}\right)}\{\hat{p} \ldots\} \cdot \beta 2 p_{\mu} \cdot d_{\mu \lambda}(p-k) ; \\
p_{\mu} \cdot d_{\mu \lambda}(p-k)=O\left(k_{\perp}\right) .
\end{gathered}
$$

Consequently, it is the ladder contribution of Fig. 1.4 alone which generates the quark $\rightarrow$ quark+gluon splitting kernel (1.49) (see Problem 1.2).

The advantages of such a "physical" gauge are manifested most clearly in the Yang-Mills theory 9 . In QCD the gluon, like the quark, has a color charge and therefore can itself emit nonladder gluons (see Fig. 1.6). Additional complications arise also from the 4-gluon coupling and ghosts. Therefore selection of the (ghostless) gauge (1.50) which makes it possible to reduce the set of contributing Feynman diagrams to the ladders presented in Fig. 1.5 proves to be very efficient.

The field "wavefunction" of a dressed quark has a parton structure with colored quasi-real quarks and gluons of two physical polarizations acting as partons.

Following the same logic three other elementary parton decay kernels can be derived which are responsible for the $q \rightarrow g(z), g \rightarrow q(z)$ and $g \rightarrow g(z)$ transitions. When calculating these kernels one sums over the colors and polarizations of the final partons and, generally speaking, various polarization states contribute. However, after one averages over the parent parton polarizations (and colors) the polarization matrix for the partons entering the next 

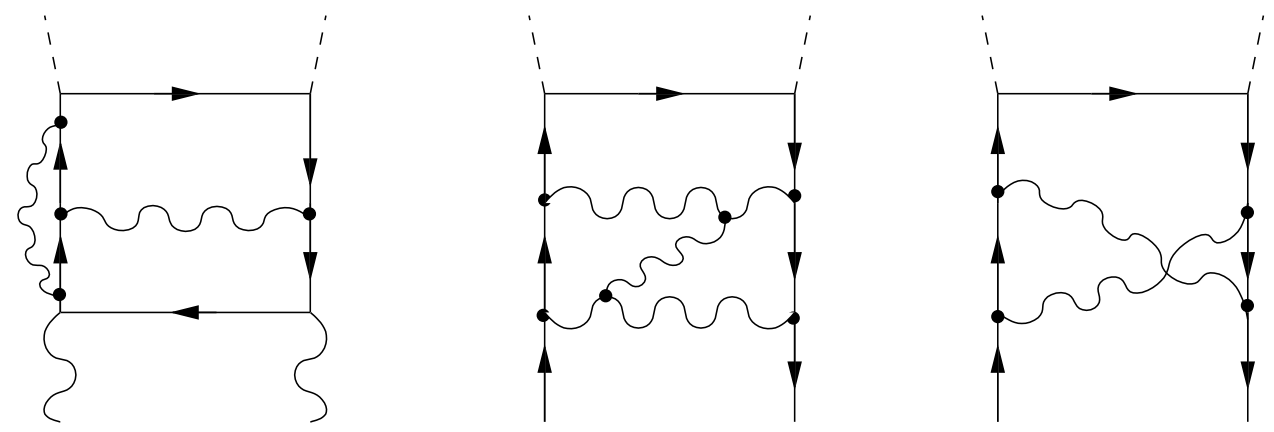

Figure 1.6: Examples of interference QCD diagrams contributing to DIS structure functions in the Feynman gauge.

cell appears to be again that of the unpolarized target, and one can repeat the same procedure for the next cell, and so forth (see Problems at the end of the Chapter).

\subsubsection{Renormalization of the Ladder and QCD Form Factors}

An attentive reader might notice that owing to the singularity of the kernel $\Phi_{F}^{F}$ at $z=1$ (as well as of $\Phi_{G}^{G}$, see below) the ladder $\beta$-integrals diverge logarithmically, $c f$. (1.44). This corresponds to the fact that the total probability of soft gluon emission is infinite. However, the probability that such a gluon will be absorbed by the same parton that has emitted it, diverges similarly. The final result for the structure functions should be finite.

It is worthwhile to mention that, e.g., the total $e^{+} e^{-}$annihilation cross section contains no logs at all, neither "soft" nor "collinear", and can be ex- 
pressed in terms of a PT-series in powers of $\alpha_{s}$. Such a complete cancellation of real and virtual large logarithmic contributions in the total sum of separate exclusive channels is in line with the well-known Kinoshita-Lee-Nauenberg theorem 18 .

The statement of this general theorem is no longer valid formally when one turns to inclusive particle distributions from $e^{+} e^{-}$annihilation or to the DIS cross section. However, soft gluon singularities are still canceled.

The physical explanation of this phenomenon is clear: soft radiation does not influence the kinematics of the radiating quark, and if one makes no arrangement to register the emitted $g$ or to restrict significantly its phase space, then the probability of such an inclusive process must be independent of the quark ability to emit such an "invisible" gluon. Thus the double log divergences cancel in the total sum of all possible exclusive channels (with and without soft gluon emissions). Only collinear logs (mass singularities) survive, that describe the recoil effects produced on the radiating quark by hard gluon emissions, giving rise to the scaling violation.

Soft gluon reabsorption processes must be included in the virtual corrections to parton propagators and interaction vertices. Thus we come to the problem of renormalization of the LLA ladder. With account of virtual corrections to the ladder elements, propagators and interaction vertices acquire renormalization factors depending on parton virtualities. This dependence turns out to be logarithmic, so that virtual effects must be taken into full account on equal footing with the ladder cells describing elementary parton decays.

It is complexity of the renormalization program, which one has to pay with for the transparent probabilistic structure of contributing Feynman diagrams in a physical gauge. An auxiliary vector $q^{\prime}$, used to define the axial gauge (1.50), induces a dependence of the renormalization functions on the product $\left(k \cdot q^{\prime}\right)$, in addition to the usual dependence on the square of the 
virtual momentum $k^{2}$. Thus the formal realization of the program becomes cumbersome 13. The result, however, proves to be very simple and natural: after having suppressed interference graphs one gets the possibility to interpret in a probabilistic manner not only successive parton branchings but the reabsorption effects as well.

\subsubsection{Running Coupling in Parton Cascades}

In the LLA we are looking for all sources of $\operatorname{logarithmic} Q^{2}$ dependence. One standard source is well known: ultraviolet divergences inherent to a field theory with dimensionless coupling force the finite renormalized coupling "constant" to run logarithmically with $Q^{2}$. Since it is the virtuality of the photon that sets momentum scale of the DIS problem, one could get rid of ultraviolet renormalization by choosing $\alpha_{s}\left(Q^{2}\right)$ as the PT expansion parameter. However, due to strong transverse momentum ordering (1.16), (1.42) inside the multiparton LLA-ladder characteristic distances are large compared to $1 / Q$ and one might expect smaller momentum scales to enter the game resulting in larger effective strength of parton-parton interaction.

Field theory textbooks relate the effective coupling to the renormalized vertex of particle interaction with (large) space-like momenta of the same order of magnitude. For the $q \bar{q} g$ vertex, e.g., one has

$$
\begin{aligned}
\alpha_{s}\left(\mu^{2}\right)= & \alpha_{s}^{(0)} \cdot \sqrt{Z_{q}\left(k_{1}\right)} \sqrt{Z_{q}\left(k_{2}\right)} \sqrt{Z_{g}\left(k_{3}\right)} \cdot \Gamma_{q \bar{q} g}\left(k_{1}, k_{2}, k_{3}\right) \\
& k_{1}^{2} \sim k_{2}^{2} \sim k_{3}^{2} \sim-\mu^{2}
\end{aligned}
$$

with $Z, \Gamma$ the wavefunction and vertex renormalization functions, $\alpha_{s}^{(0)}$ the bare coupling.

However, the parton kinematics we have inside a DIS parton ladder look very different from (1.55) since two of the partons (incoming $k_{1}$ and outgoing $k_{2}$ 
vertical lines) have space-like strongly ordered virtualities, and the third one (horizontal ladder rung $k_{3}$ ) we dealt with as an on-mass-shell particle:

$$
k_{1}^{2}, k_{2}^{2}<0, \quad\left|k_{2}^{2}\right| \sim k_{\perp_{2}}^{2} \gg\left|k_{1}^{2}\right| \sim k_{\perp_{1}}^{2} ; \quad k_{3}^{2} \gtrsim 0 .
$$

To understand how the running coupling emerges in such a specific situation it is worthwhile to step back to a simpler QED example.

\section{Wavefunction renormalization and parton form factors}

Since the QED coupling is numerically small, one can hardly see in reality any manifestation of its running behavior. Imagine, however, QED with two charged fermions: an electron and a parametrically heavy "muon" to look for signs of $\alpha\left(m_{\mu}^{2}\right)>\alpha_{0} \approx \frac{1}{137}$ in hard processes involving such muons.

Let us consider the QED decay of "muonium" bound states $M=\mu^{+} \mu^{-}$ and ask the question, what is the momentum scale which determines the argument of $\alpha$ entering expressions for decay widths

$$
\Gamma_{M}=\mathrm{C} \cdot \alpha^{2}(?)
$$

of $C$-odd $\left(M_{-} \rightarrow e^{+} e^{-}\right)$and C-even $\left(M_{+} \rightarrow 2 \gamma\right)$ states shown in Fig. 1.7. To answer the question about the argument of $\alpha$ means to keep track of higher order logarithmic corrections $\sim\left(\alpha_{0} \log m_{\mu}^{2}\right)^{n}$ to the Born width $\Gamma \propto \alpha_{0}^{2}$.

Neglecting for the sake of simplicity $\alpha \log \alpha$ effects one can relate the characteristic size of the bound state to the Compton wave length $1 / m \mu$ of a massive lepton and to expect thus $\alpha\left(m_{\mu}^{2}\right)$ to appear in (1.56).

$M_{-} \rightarrow e^{+} e^{-}$decay and the running coupling. It is straightforward to approve this expectation for the case of $M_{-}$decay of Fig. 1.7 h. The total 

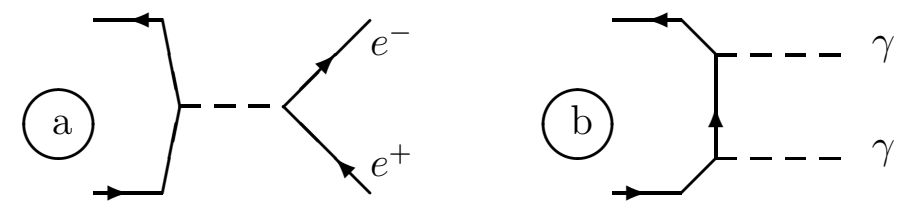

Figure 1.7: Born amplitudes of $C$-odd (a) and $C$-even (b) "muonium" decays.

decay width here can be obtained directly by calculating discontinuity of the photon renormalization function $Z_{3}$ at positive virtuality $M^{2} \approx\left(2 m_{\mu}\right)^{2}$

$$
\begin{aligned}
& \Gamma_{M_{-} \rightarrow e^{+} e^{-}} \propto \alpha_{0} \operatorname{Im} Z_{3}\left(M^{2}\right) \approx \alpha_{0} \cdot Z_{3}\left(M^{2}\right) \operatorname{Im} \Sigma\left(M^{2}\right) Z_{3}^{*}\left(M^{2}\right) . \\
& \operatorname{Im} Z_{3}=\sum_{\text {loops cuts }} \sum_{1}
\end{aligned}
$$

Extracting the $\alpha_{0}$ factor from the imaginary part of the photon self-energy $\Sigma$ and replacing (in the logarithmic approximation) $Z_{3}\left(M^{2}\right)$ by the renormalization function at Eucledean momentum

$$
Z_{3}\left(M^{2}\right) Z_{3}^{*}\left(M^{2}\right) \approx Z_{3}^{2}\left(-M^{2}\right)
$$

one obtains

$$
\Gamma_{M_{-} \rightarrow e^{+} e^{-}} \propto\left(\alpha_{0} Z_{3}\left(-M^{2}\right)\right)^{2} \cdot \Phi_{\gamma} \quad \propto \alpha^{2}\left(m_{\mu}^{2}\right)
$$

with $\Phi_{\gamma}$ the constant connected to the total probability of $\gamma^{*} \rightarrow e^{+} e^{-}$splitting (cf. (1.87c) and Problem 1.4 below):

$$
\begin{aligned}
\operatorname{Im} \Sigma & =\frac{1}{4} \alpha_{0} \cdot \Phi_{\gamma} \\
\Phi_{\gamma} & =\int_{0}^{1} d z 2\left[z^{2}+(1-z)^{2}\right]=\frac{4}{3} .
\end{aligned}
$$


The quantity $\Phi_{\gamma}$ itself determines the logarithmic behavior of $Z_{3}$ and thus the running QED coupling. Indeed, let us consider the dispersion relation with one subtraction for the photon propagator (choosing standard normalization $Z_{3}(0)=1$ for the real photon)

$$
Z_{3}\left(k^{2}\right)=1+\frac{k^{2}}{\pi} \int_{\left(2 m_{e}\right)^{2}}^{\infty} \frac{d m^{2} \operatorname{Im} Z_{3}\left(m^{2}\right)}{m^{2}\left(m^{2}-k^{2}-i \epsilon\right)} .
$$

Then for large negative $k^{2}=-\kappa^{2}$ the dominant contribution to (1.61) will come from logarithmic integration over the region of virtual photon masses $m_{e}^{2} \ll m^{2} \ll \kappa^{2}$ and making use of (1.58), (1.60a) one arrives at

$$
\frac{\alpha\left(\kappa^{2}\right)}{\alpha_{0}} \equiv Z_{3}\left(-\kappa^{2}\right) \approx \int_{4 m_{e}^{2}}^{\kappa^{2}} \frac{d m^{2}}{m^{2}} Z_{3}\left(-m^{2}\right) \cdot \frac{\alpha\left(m^{2}\right)}{4 \pi} \Phi_{\gamma}
$$

which leads immediately to the well known result for the first loop QED $\beta$-function

$$
\frac{\partial}{\partial \ln \kappa^{2}} \frac{\alpha\left(\kappa^{2}\right)}{4 \pi}=\Phi_{\gamma} \cdot\left(\frac{\alpha\left(\kappa^{2}\right)}{4 \pi}\right)^{2}, \quad \Phi_{\gamma}=\frac{4}{3} \cdot n_{f}
$$

with $n_{f}$ the number of light fermion generations $\left(n_{f}=1\right.$ in our toy model). Thus for large negative virtualities the photon renormalization function can be related directly to the elementary "parton" splitting as

$$
Z_{3}\left(-\kappa^{2}\right)=\exp \left\{\int^{\kappa^{2}} \frac{d m^{2}}{m^{2}} \frac{\alpha\left(m^{2}\right)}{4 \pi} \Phi_{\gamma}\right\} .
$$

$M_{+} \rightarrow \gamma \gamma$ decay and the photon "form factor". Now we turn to the second process of the $M_{+}$state decay displayed in Fig. 1.7. Here however 
expectation similar to (1.59) seems to fail: the two photon decay probability does not acquire $\alpha_{0} \log m_{\mu}^{2}$ corrections we are looking for: the large value of $m_{\mu}$ affects the $\mu \mu \gamma$ vertices and virtual $\mu$ propagators which effects cancel because of the Ward identity $\left(Z_{1}^{-1} Z_{2}=1\right)$ and the width remains unchanged:

$$
\Gamma_{M_{+} \rightarrow \gamma \gamma}=C_{+} \cdot \alpha_{0}^{2}
$$

Then what about an intuitive connection between the effective interaction strength and characteristic distances? It is recovered when one considers the total decay width of which the two- $\gamma$ channel appears to be a small fraction at large muon masses. Additional decay channels are those with light electrons in the final state. To take them into account one has to integrate the product of discontinuities of the two photons over virtual photon masses $m_{\gamma}$ that are kinematically allowed in the decay process. These integrals are logarithmic up to $m_{\gamma}^{2} \lesssim m_{\mu}^{2}$ and end up with replacement of each of two $\alpha_{0}$ in the expression for the width by $\alpha\left(m_{\mu}^{2}\right)$ due to (1.62).

This result can be interpreted in terms of cascading picture. Since it is $m_{\mu}^{2}$ to set a natural momentum scale of our problem let us define our "partons", photons and electrons, to be pointlike at this scale. This means redefining the field wavefunction renormalization factors $Z_{3} \equiv Z_{\gamma}$ and $Z_{2} \equiv Z_{e}$ to be unity at that virtuality level:

$$
Z_{\gamma}\left(-m_{\mu}^{2}\right)=Z_{e}\left(-m_{\mu}^{2}\right)=1
$$

Then for an arbitrary momentum scale $\kappa^{2}<m_{\mu}^{2}$ we have according to (1.64)

$$
Z_{\gamma}\left(-\kappa^{2}\right)=\exp \left\{-\int_{\kappa^{2}}^{\mu^{2}} \frac{d m^{2}}{m^{2}} \frac{\alpha\left(m^{2}\right)}{4 \pi} \Phi_{\gamma}\right\} .
$$

In field theory any particle is a mixture of Fock state components consisting of a different number of bare particles (partons). $Z_{\gamma}\left(-\kappa^{2}\right)$ physically gives the 
probability to find a single bare photon inside the photon field wavefuction when probed with spatial resolution $\Delta \rho \sim 1 / \kappa$. So when looking for the exclusive channel of production of two real photons one has to multiply the total decay width by two photon "form factors" $Z_{\gamma}(0)$ to ensure that photons emitted in a hard process of characteristic scale $m_{\mu}^{2}$ did not decay afterwards producing $e^{+} e^{-}$pairs, secondary photons etc.

$$
\begin{aligned}
\Gamma_{M_{+}}^{t o t} & =C_{+} \alpha^{2}\left(m_{\mu}^{2}\right), \\
\Gamma_{M_{+} \rightarrow \gamma \gamma}^{e x c l} & =\Gamma_{M_{+}}^{t o t} Z_{\gamma}^{2}(0)=C_{+} \alpha^{2}\left(m_{\mu}^{2}\right)\left[\frac{\alpha\left(m_{\mu}^{2}\right)}{\alpha_{0}}\right]^{-2}=C_{+} \alpha_{0}^{2} .
\end{aligned}
$$

The same holds true for the first process with the $e^{+} e^{-}$pair produced after the decay of $M_{-}$"muonium". Here to obtain the probability of exclusive pair production cross section we have to multiply the modified Born probability by the product of two electron form factors

$$
Z_{e}\left(-\kappa^{2}\right)=\exp \left\{-\int_{\kappa^{2}}^{\mu^{2}} \frac{d m^{2}}{m^{2}} \frac{\alpha\left(m^{2}\right)}{4 \pi} \Phi_{e}\right\}
$$

where $\Phi_{e}$, similarly to (1.60b), is related to the $e \rightarrow e \gamma$ emission probability

$$
\Phi_{e}=\int_{0}^{1} d z 2 \frac{1+z^{2}}{1-z} .
$$

The result would be zero, of course, because of famous infrared "catastrophe": (1.69) diverges to produce an infinite suppression of the exclusive cross section since it is impossible to get charged particles in the final states not being accompanied by soft photons.

$$
\begin{aligned}
\Gamma_{M_{-}}^{t o t} & =C_{-} \Phi_{\gamma} \alpha^{2}\left(m_{\mu}^{2}\right), \\
\Gamma_{M_{-} \rightarrow e^{+} e^{-}}^{e x c l} & =\Gamma_{M_{-}}^{t o t} \cdot Z_{e}^{2}(0)=0 .
\end{aligned}
$$




\subsubsection{Virtual and Inclusive corrections to a ladder cell}

Now let us come back to a DIS ladder cell with parton $A$ splitting into $B+C$ where $C$ has a time-like momentum (horizontal ladder rung). It is the virtuality of the space-like $B$ momentum $-k_{B}^{2} \sim k_{\perp}^{2}$ that determine the characteristic "hardness" of the parton splitting process.

As far as structure functions are concerned, parton $C$ is allowed to evolve in the final state producing its own subjet. This evolution is restricted by the condition $k_{C}^{2}<k_{\perp}^{2}$ to preserve the main logarithmic integration over $k_{\perp}$ in the cell. Integrating over the virtual mass of $C$ we obtain similarly to the QED example

$$
\begin{gathered}
\frac{1}{\pi} \int^{k_{\perp}^{2}} \frac{d k_{C}^{2}}{k_{C}^{2}} \operatorname{Im}\left\{\Gamma_{A B C}\left(k_{A}^{2}, k_{B}^{2}, k_{C}^{2}\right) d_{C}\left(k_{C}^{2}\right) \Gamma_{A B C}\left(k_{A}^{2}, k_{B}^{2}, k_{C}^{2}\right)\right\} \\
\approx \Gamma_{A B C}^{2}\left(k_{A}^{2},-k_{\perp}^{2},-k_{\perp}^{2}\right) d_{C}\left(-k_{\perp}^{2}\right) .
\end{gathered}
$$

In general one has to consider as well logarithmic contributions coming from virtual gluon lines embracing a few ladder cells. In physical gauges, however, those are absent (for the same reason as non-ladder interference diagrams discussed above), so that one is left with local virtual corrections to parton propagators $d\left(k^{2}\right)$ and interaction vertices $\Gamma\left(k_{A}^{2}, k_{B}^{2}, k_{C}^{2}\right)$ only. It is in a physical gauge therefore where renormalization functions $d_{A}\left(k^{2}\right)$ acquire clear meaning of parton form factors.

The last important property to be mentioned 13 is the independence of vertex functions $\Gamma$ in (1.71) of the smallest virtuality $k_{A}^{2}\left(\right.$ no $\log \left(\left|k_{A}^{2}\right| / k_{\perp}^{2}\right)$ terms singular at $k_{A}^{2} \rightarrow 0$ ). For this reason, replacing $k_{A}^{2}$ by $-k_{\perp}^{2}$ in the vertex, one can construct the product that determines in a usual way the running coupling ( $c f .(1.55)$ ):

$$
\left.\alpha_{s}^{2} d_{A}\left(k_{A}^{2}\right) d_{B}\left(k_{B}^{2}\right) d_{C}\left(k_{C}^{2}\right) \Gamma_{A B C}^{2}\left(k_{A}^{2}, k_{B}^{2}, k_{C}^{2}\right)\right|_{k_{A}^{2} \sim k_{B}^{2} \sim k_{C}^{2} \sim-k_{\perp}^{2}}=\alpha_{s}^{2}\left(k_{\perp}^{2}\right) .
$$


Each new interaction in the parton ladder introduces two " $B$ "-lines, one " $C$ " and two $\Gamma$ factors. Extracting from the product of corresponding renormalization functions the combination (1.72) one finally obtains for the renormalized cell

$$
d w_{A}^{B(+C)}=\frac{d z}{z} \Phi_{A}^{B}(z) \frac{d k_{\perp}^{2}}{k_{\perp}^{2}} \frac{\alpha_{s}\left(k_{\perp}^{2}\right)}{4 \pi} d_{B}\left(-k_{\perp}^{2}\right)\left(d_{A}\left(-k_{\perp}^{2}\right)\right)^{-1} .
$$

Building up a ladder one starts from a parton $A$ with finite virtuality $k_{0}^{2}<0$ and one has to multiply the forward scattering amplitude by the wavefunction renormalization factors of the incoming parton $\left(\sqrt{d_{A}\left(k_{0}^{2}\right)}\right)^{2}$. The first splitting $k_{0} \rightarrow k_{1}$, according to (1.73), induces the ratio of renormalization functions $d_{B}\left(-k_{\perp 1}^{2}\right) / d_{A}\left(-k_{\perp 1}^{2}\right)$.

In what follows we shall attribute the $d_{B}\left(-k_{\perp_{i}}^{2}\right)$ factors to the upper part of the ladder, ascribing the ratio $d_{A}\left(-k_{\perp_{i-1}}^{2}\right) / d_{A}\left(-k_{\perp i}^{2}\right)$ to the ladder cell \#i itself. (To simplify notations we shall ommit the minus sign in the argument of $d$ hereafter.)

\subsection{DGLAP Evolution Equations}

In this Section we derive the Evolution Equations governing the development of partonic systems in the LLA. We shall present analytic formulae for inclusive parton distributions and discuss their features in some practically important and physically interesting limits. Separate subsection will be devoted to displaying the nice symmetry properties of parton splitting functions. 


\subsubsection{Evolution Equations in an Integral Form}

Let us take as a target particle a parton $A$ of typical transverse size $\sim 1 / k_{0}$ (or characteristic virtuality $\left.\left|k^{2}\right| \sim k_{0}^{2}\right)$ and denote by $D_{A}^{B}\left(x, Q^{2}, k_{0}^{2}\right)$ the probability to find inside the parton cloud of the "dressed" particle $A$ a parton of species $B$ with longitudinal momentum fraction $x$ and arbitrary transverse momentum (virtuality) up to $Q^{2}$. Summation (averaging) over polarizations and colors of the field $B(A)$ is assumed. We consider on equal footing distributions of quarks, antiquarks and gluons as partons in the dressed quarks, antiquarks and gluons, so that $A, B=q, \bar{q}, g$ or $A, B=F, G$.

Ladder-type diagrams can be easily summed up with the help of the BetheSalpeter-type technique, treating single ladder cells (two-particle irreducible blocks) as kernels of an integral equation. This can be done in two ways, iterating the ladder rungs of Fig. 1.5] either from "above" or from "below".

The Bethe-Salpeter equations, one obtains applying the latter method, have the following analytic form:

$$
\begin{aligned}
D_{A}^{B}\left(x, q^{2}, k_{0}^{2}\right)= & \delta_{A}^{B} \delta(1-x) d_{A}\left(k_{0}^{2}\right)+d_{A}\left(k_{0}^{2}\right) \sum_{C} \int_{k_{0}^{2}}^{Q^{2}} \frac{d k_{\perp}^{2}}{k_{\perp}^{2}} \int_{0}^{1} \frac{d z}{z} \\
& \frac{\alpha_{s}\left(k_{\perp}^{2}\right)}{4 \pi} d_{A}^{-1}\left(k_{\perp}^{2}\right) \Phi_{A}^{C}(z) D_{C}^{B}\left(\frac{x}{z}, Q^{2}, k_{\perp}^{2}\right) .
\end{aligned}
$$

The first term in the right hand side is the Born term which corresponds to "measuring" the target parton $A$ without any other particles present in the partonic wavefunction. The probability of finding such an unnatural fluctuation is suppressed by the Sudakov factor $d_{A}\left(k_{0}^{2}\right)$. This suppression should become stronger with increase of the gap between $Q^{2}$ and $k_{0}^{2}$. The integral term displays the first parton splitting $A \rightarrow C(z)$ followed by subsequent evolution which now looks like the DIS off the new target particle $C$ with 
characteristic transverse size $\sim 1 / k_{\perp} \ll 1 / k_{0}$. To make it sure that the parton splitting we single out is really the very first one we have to forbid any possible branching of $A$ in between the two scales $1 / k_{0}$ and $1 / k_{\perp}$. It is the ratio of the two $d$-factors, $d_{A}\left(k_{0}^{2}\right) / d_{A}\left(k_{\perp}^{2}\right)$, that accounts for this form factor suppression in the integral term of (1.74). The Bethe-Salpeter equations determine both unknown quantities $d_{F, G}$ and $D_{A}^{B}$ which are closely related to each other.

We start with extracting the $d_{A}$-functions. To do this, let us invoke the energy-momentum conservation sum rule, i.e. the condition that all partons inside a dressed particle carry, in the aggregate, the total momentum of the target (see, e.g., Ref. [19]):

$$
\sum_{B} \int_{0}^{1} D_{A}^{B}\left(x, Q^{2}, k_{0}^{2}\right) x d x=1 .
$$

Multiplying (1.74) by $x$ and integrating, we arrive at the following equation for the parton renormalization functions:

$$
1=d_{A}\left(k_{0}^{2}\right)+d_{A}\left(k_{0}^{2}\right) \sum_{C} \int_{k_{0}^{2}}^{Q^{2}} \frac{d k_{\perp}^{2}}{k_{\perp}^{2}} \int_{0}^{1} z d z \frac{\alpha_{s}\left(k_{\perp}^{2}\right)}{4 \pi} \Phi_{A}^{C}(z) d_{A}^{-1}\left(k_{\perp}^{2}\right) .
$$

Differentiating with respect to $\ln k_{0}^{2}$ we get

$$
\frac{\partial}{\partial \ln k_{0}^{2}} d_{A}^{-1}\left(k_{0}^{2}\right)=-\frac{\alpha_{s}\left(k_{0}^{2}\right)}{4 \pi} \sum_{C} \int_{0}^{1} z d z \Phi_{A}^{C}(z) \cdot d_{A}^{-1}\left(k_{0}^{2}\right) .
$$

The $z$-integrals in (1.76) and (1.77) formally diverge at $z=1$, showing thus that it is the quark and gluon form factors that contain double logarithmic singularities. 


\subsubsection{Cancellation of Soft Divergences in Differential Evolution Equations}

Now we multiply (1.74) by $d_{A}^{-1}\left(k_{0}^{2}\right)$ and differentiate it to obtain

$$
\begin{gathered}
d_{A}^{-1}\left(k_{0}^{2}\right) \frac{\partial}{\partial \ln k_{0}^{2}} D_{A}^{B}\left(x, Q^{2}, k_{0}^{2}\right)+D_{A}^{B}\left(x, Q^{2}, k_{0}^{2}\right) \frac{\partial}{\partial \ln k_{0}^{2}} d_{A}^{-1}\left(k_{0}^{2}\right) \\
=-d_{A}^{-1}\left(k_{0}^{2}\right) \frac{\alpha_{s}\left(k_{0}^{2}\right)}{4 \pi} \sum_{C} \int_{0}^{1} z d z \Phi_{A}^{C}(z) D_{C}^{B}\left(\frac{x}{z}, Q^{2}, k_{0}^{2}\right)
\end{gathered}
$$

Combining with (1.77) we arrive at the Master Equation for parton distributions:

$$
\begin{gathered}
\frac{\partial}{\partial \ln k_{0}^{2}} D_{A}^{B}\left(x, Q^{2}, k_{0}^{2}\right)=-\frac{\alpha_{s}\left(k_{0}^{2}\right)}{4 \pi} \sum_{C} \int_{0}^{1} \frac{d z}{z} \Phi_{A}^{C}(z) \\
\cdot\left[D_{C}^{B}\left(\frac{x}{z}, Q^{2}, k_{0}^{2}\right)-z^{2} D_{A}^{B}\left(x, Q^{2}, k_{0}^{2}\right)\right]
\end{gathered}
$$

which is now free from soft divergencies. Indeed, the singular kernels are only those with $C=A$ (which correspond to a gluon emission in the $s$-channel), and the $D$-functions in square brackets cancel at $z \rightarrow 1$ damping the singularity. Thus, as we expected, no soft gluons show up in the measurable parton distributions $D_{A}^{B}$.

Similar system of Bethe-Salpeter equations can be derived by iterating the ladder from above. Picking up the very last cell where the parton $B$ interacts with the external $Q^{2}$-probe ("partonometer"), we proceed in full analogy with 
the derivation of (1.79) to get an alternative form of the Evolution Equations:

$$
\begin{gathered}
\frac{\partial}{\partial \ln Q^{2}} D_{A}^{B}\left(x, Q^{2}, k_{0}^{2}\right)=\frac{\alpha_{s}\left(Q^{2}\right)}{4 \pi} \sum_{C} \int_{0}^{1} \frac{d z}{z} \Phi_{C}^{B}(z) \\
\cdot\left[D_{A}^{C}\left(\frac{x}{z}, Q^{2}, k_{0}^{2}\right)-z^{2} D_{A}^{B}\left(x, Q^{2}, k_{0}^{2}\right)\right]
\end{gathered}
$$

As one can easily see, the LLA parton distributions prove to depend not on $k_{0}^{2}$ and $Q^{2}$ separately, but rather on a definite combination of virtualities. Introducing a new variable 4 according to

$$
d \xi\left(k^{2}\right) \equiv \frac{\alpha_{s}\left(k^{2}\right)}{4 \pi} \frac{d k^{2}}{k^{2}} \quad, \quad \xi\left(Q^{2}\right)=\int_{\mu^{2}}^{Q^{2}} \frac{d k^{2}}{k^{2}} \frac{\alpha_{s}\left(k^{2}\right)}{4 \pi},
$$

we note, that $D_{A}^{B}$-functions actually depend on the difference $\Delta \xi$. Thus

$$
\begin{aligned}
D_{A}^{B}\left(x, Q^{2}, k_{0}^{2}\right) & =D_{A}^{B}(x, \Delta \xi), \\
\Delta \xi=\xi\left(Q^{2}\right)-\xi\left(k_{0}^{2}\right) & \approx \frac{1}{b} \ln \frac{\alpha_{s}\left(k_{0}^{2}\right)}{\alpha_{s}\left(Q^{2}\right)}=\frac{1}{b} \ln \frac{\ln \left(Q^{2} / \Lambda^{2}\right)}{\ln \left(k_{0}^{2} / \Lambda^{2}\right)}
\end{aligned}
$$

where we used the one-loop expression for the running coupling to derive the analytic form of $\Delta \xi$. In terms of this variable (1.79) and (1.80) in fact coincide. Variable $\xi$ can be treated as the "evolution time" with the matrix $\Phi$ playing the role of the "Hamiltonian" of the system. We shall come back to this analogy a little later, but now let us present the resulting Evolution Equation in the integral form, which incorporates (1.79), (1.80) and satisfies appropriate 
initial conditions following from the original Bethe-Salpeter equation (1.74)

$$
\begin{aligned}
D_{A}^{B}(x, \xi) & =\delta_{A}^{B} \delta(1-x)+\sum_{C} \int_{0}^{\xi} d \xi^{\prime} \int_{0}^{1} \frac{d z}{z} \Phi_{C}^{B}(z)\left[D_{A}^{C}\left(\frac{x}{z}, \xi^{\prime}\right)-z^{2} D_{A}^{B}\left(x, \xi^{\prime}\right)\right] \\
& =\sum_{C} \int_{0}^{\xi} d \xi^{\prime} \int_{0}^{1} \frac{d z}{z} \Phi_{A}^{C}(z)\left[D_{C}^{B}\left(\frac{x}{z}, \xi^{\prime}\right)-z^{2} D_{A}^{B}\left(x, \xi^{\prime}\right)\right]
\end{aligned}
$$

\subsubsection{Splitting Functions and Relations between them}

The elementary parton splitting functions we are considering here describe decay probabilities averaged (summed up) over colors and polarizations of parent (offspring) partons, which play the role of kernels of evolution equations (1.83) for the LLA parton distributions. Let us recall that the decay phase space for the space-like parton wavefunction evolution determining the DIS structure functions is

$$
d w^{A \rightarrow B+C}=\frac{d k_{\perp}^{2}}{k_{\perp}^{2}} \frac{\alpha_{s}\left(k_{\perp}^{2}\right)}{4 \pi} \frac{d z}{z} \Phi_{A}^{B C}(z)
$$

with $z$ the longitudinal momentum fraction carried by the parton $B$.

Notice, that the same functions $\Phi_{A}^{B C}$ are responsible also for decays of partons with positive momenta squared, i.e. the time-like evolution that determine internal structure of QCD jets produced, e.g., in $e^{+} e^{-}$annihilation. In this case the longitudinal phase space turns out to be symmetric and the

\footnotetext{
${ }^{4}$ corresponding functions for fixed polarization states and discussion of their symmetry properties can be found in Refs. 812
} 
differential decay probability reads

$$
d w^{A \rightarrow B+C}=\frac{d k_{\perp}^{2}}{k_{\perp}^{2}} \frac{\alpha_{s}\left(k_{\perp}^{2}\right)}{4 \pi} d z \Phi_{A}^{B C}(z) .
$$

It is convenient for discussion of the relations between kernels to extract color factors

$$
\begin{gathered}
t_{i j}^{a} t_{j k}^{a}=C_{F} \delta_{i k}, \quad t_{i k}^{a} t_{k i}^{b}=T_{R} \delta^{a b}=\frac{1}{2} \delta^{a b}, \quad f_{a d e} f_{b d e}=N_{c} \delta_{a b} ; \\
\Phi_{F}^{F}(z)=C_{F} V_{F}^{F}(z), \quad \Phi_{F}^{G}(z)=C_{F} V_{F}^{G}(z) \\
\Phi_{G}^{F}(z)=T_{R} V_{G}^{F}(z), \quad \Phi_{G}^{G}(z)=N_{c} V_{G}^{G}(z) .
\end{gathered}
$$

The splitting functions then take the form 899 .

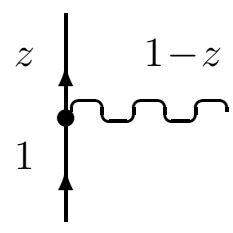

$$
\begin{gathered}
V_{F}^{F}(z)=2 \frac{1+z^{2}}{1-z} \\
V_{F}^{G}(z)=2 \frac{1+(1-z)^{2}}{z}
\end{gathered}
$$




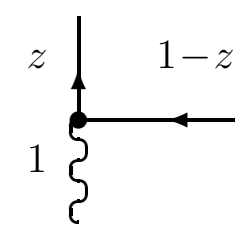

$$
\begin{gathered}
V_{G}^{F}(z)=2\left[z^{2}+(1-z)^{2}\right] \\
V_{G}^{G}(z)=4\left[z(1-z)+\frac{1-z}{z}+\frac{z}{1-z}\right] .
\end{gathered}
$$$$
z\left\{\begin{array}{l}
1-z \\
\text { nu }
\end{array}\right.
$$

Here we list the most important symmetry properties of parton splitting functions.

Parton Exchange results in an obvious relation between probabilities to find decay products with complementary momenta fractions:

$$
V_{A}^{B C}(z)=V_{A}^{C B}(1-z) .
$$

A Crossing Relation [6 emerges when one links together two splitting processes corresponding to opposite evolution "time" sequences:

$$
V_{A}^{B}\left(\frac{1}{z}\right)=(-1)^{2 s_{A}+2 s_{B}-1} \frac{1}{z} V_{B}^{A}(z)
$$

with $s_{A}$ the spin of the particle $A$.

The Super-Symmetry Relation 9 exploits the existence of the supersymmetric field theory closely related to real QCD:

$$
V_{F}^{F}(z)+V_{F}^{G}(z)=V_{G}^{F}(z)+V_{G}^{G}(z) .
$$


Conformal Invariance 12 of the leading twist approximation leads to a number of relations between splitting functions, the simplest of which reads

$$
\left(z \frac{d}{d z}-2\right) V_{G}^{F}(z)=\left(z \frac{d}{d z}+1\right) V_{F}^{G}(z) .
$$

As we see, these relations leave not much freedom for splitting functions. In fact, one could borrow $V_{F}^{F}$ from QED text books ( $c f$. (1.6) ) and reconstruct successively $V_{F}^{G}$ (with use of (1.88) $), V_{G}^{F}$ (1.89), and then even the gluon selfinteraction $V_{G}^{G}$ from (1.90).

The general character of the symmetry properties makes them practically useful when studying next-to-leading corrections to anomalous dimensions and coefficient functions where one faces technically difficult calculations. For example, the super-symmetric QCD analog had been used to choose between two contradictory calculations for the two-loop anomalous dimensions (see, e.g., Ref. [15]). We illustrate the idea by another example of the next-toleading result, i.e. the ratio of parton multiplicities in gluon and quark jets which reads 17

$$
R \frac{\mathcal{N}_{g}}{\mathcal{N}_{q}}=1-\frac{\gamma_{0}}{6}\{1+T(1-2 R)\}+\frac{1}{8}\left(\frac{\gamma_{0}}{6}\right)^{2}\left\{-25(58 R-19) T+\left(6-4 R-16 R^{2}\right) T^{2}\right\}
$$

with $\gamma_{0}=\sqrt{2 N_{c} \alpha_{s} / \pi}$ the typical PT parameter which we shall meet many times below considering soft parton multiplication. $R$ and $T$ in (1.92) are the following numbers:

$$
R \equiv \frac{C_{F}}{N_{c}} \quad, \quad T \equiv \frac{2 n_{f} T_{R}}{N_{c}}
$$

In susy-QCD "quark" and "gluon" belong to the same (adjoint) representation of the color group, so that all color factors (1.86) become equal: 
$C_{F}=N_{c}=T_{R}$. Moreover, the "quark" here is the Majorana fermion (identical to the "antiquark"), so that the total number of $q \bar{q}$ flavor states becomes $2 n_{f}=1$. Then, as one can easily check, all corrections to multiplicity ratio $\mathcal{N}_{g} / \mathcal{N}_{q}=1$ vanish at $R=T=1$.

\subsubsection{Solution of Evolution Equations}

The very nature of parton cascading makes it useful to express parton distributions in terms of the Laplace-Mellin tranformation which converts multiple energy-fraction convolutions into the product of independent successive decay probabilities.

Introducing parton distributions $D_{A}^{B}(j)$ in the moment representation,

$$
D_{A}^{B}(j, \xi) \equiv \int_{0}^{1} d x x^{j-1} D_{A}^{B}(x, \xi)
$$

one arrives at the algebraic equation for the Mellin-transformed distributions:

$$
\frac{\partial}{\partial \xi} D_{A}^{B}(j, \xi)=\sum_{C}\left(\Phi_{A}^{C}(j)-\delta_{A}^{C} \phi_{C}\right) D_{C}^{B}(j, \xi)=\sum_{C}\left(\Phi_{C}^{B}(j)-\delta_{C}^{B} \phi_{C}\right) D_{A}^{C}(j, \xi)
$$

where the following notations have been used:

$$
\Phi_{A}^{C}(j) \equiv \int_{0}^{1} d z z^{j-1} \Phi_{A}^{C}(z)
$$




$$
\begin{array}{r}
\phi_{F} \equiv \int_{0}^{1} d z z\left[\Phi_{F}^{F}(z)+\Phi_{F}^{G}(z)\right]=\int_{0}^{1} d z \Phi_{F}^{F}(z) \\
\phi_{G} \equiv \int_{0}^{1} d z z\left[\Phi_{G}^{G}(z)+2 n_{f} \Phi_{G}^{F}(z)\right]=\int_{0}^{1} d z\left[z \Phi_{G}^{G}(z)+n_{f} \Phi_{G}^{F}(z)\right]
\end{array}
$$

The solution of this equation, satisfying the initial condition

$$
D_{A}^{B}(j, \xi=0)=\delta_{A}^{B},
$$

which follows from the integral Bethe-Salpeter equation, (1.83), will give us $x$-distributions with help of the inverse Mellin transformation

$$
D_{A}^{B}(x, \xi)=\int_{(\Gamma)} \frac{d j}{2 \pi i} x^{-j} D_{A}^{B}(j, \xi),
$$

where the integration contour $\Gamma$ runs along the imaginary axis to the right from all singularities of $D(j)$ in the $j$-plane $(\operatorname{Re} j>1)$. It is convenient to represent parton distributions $D_{A}^{B}$ in a symbolic matrix form as

$$
D_{A}^{B}(j, \xi)=\left(e^{\widehat{H} \xi}\right)_{A}^{B},
$$

with the "Hamiltonian" of the system

$$
\widehat{H}_{A}^{B}(j)=\Phi_{A}^{B}(j)-\delta_{A}^{B} \phi_{A} .
$$

In this representation the evolving parton state will form a vector

$$
\left(F_{N S}, F_{S}, G\right) \text {. }
$$


with the first component describing the valence quark (non-singlet distribution with respect to the flavor group), the second one - the singlet combination of flavors (sea quarks and antiquarks), and the third component corresponding to the gluon propagation. In this basis our Hamiltonian takes the form

$$
\widehat{H}=\left|\begin{array}{ccc}
\nu_{F}(j) & 0 & 0 \\
0 & \nu_{F}(j) & 2 n_{f} \Phi_{G}^{F}(j) \\
0 & \Phi_{F}^{G}(j) & \nu_{G}(j)
\end{array}\right|
$$

where we have introduced the regularized quark and gluon trajectories:

$$
\begin{aligned}
\nu_{F}(j) & \equiv \int_{0}^{1} d z\left(z^{j-1}-1\right) \Phi_{F}^{F}(z) \\
\nu_{G}(j) & \equiv \int_{0}^{1} d z\left[\left(z^{j-1}-z\right) \Phi_{G}^{G}(z)-n_{f} \Phi_{G}^{F}(z)\right],
\end{aligned}
$$

Explicit formulae for (1.103) can be written in terms of standard $\psi$-function:

$$
\psi(j)=\frac{d}{d j} \ln \Gamma(j) ; \quad \psi(j+1)=\psi(j)+\frac{1}{j}, \quad \psi(1)=-\gamma_{E}
$$

with $\gamma_{E} \approx 0.5772$ the Euler constant. Together with Mellin-transformed $F \rightarrow$ 
$G$ and $G \rightarrow F$ kernels this gives

$$
\begin{aligned}
\nu_{F}(j) & =-C_{F}\left[4 \psi(j+1)+4 \gamma_{E}-3-\frac{2}{j(j+1)}\right], \\
\nu_{G}(j) & =-4 N_{c}\left[\psi(j+1)+\gamma_{E}\right]+\frac{11 N_{c}}{3}-\frac{2 n_{f}}{3}+\frac{8 N_{c}\left(j^{2}+j+1\right)}{j\left(j^{2}-1\right)(j+2)}, \\
\Phi_{F}^{G}(j) & =2 C_{F} \frac{j^{2}+j+2}{j\left(j^{2}-1\right)}, \\
\Phi_{G}^{F}(j) & =\frac{j^{2}+j+2}{j(j+1)(j+2)} .
\end{aligned}
$$

As we see from (1.102), the valence quark "propagates" along the trajectory $\nu_{F}(j)$, while sea-quarks mix with the gluon state. Diagonalization of the Hamiltonian results in the following "eigenfrequencies":

$$
\nu_{ \pm}=\frac{1}{2}\left\{\nu_{F}(j)+\nu_{G}(j) \pm \sqrt{\left[\nu_{F}(j)-\nu_{G}(j)\right]^{2}+8 n_{f} \Phi_{F}^{G}(j) \Phi_{G}^{F}(j)}\right\} .
$$

The same results for integer values of $j$ can be obtained by means of the OPE-RG technique 1119.

Solving (1.94) one obtains trajectories $\nu(j)$ (anomalous dimensions) determining the $\xi$-evolution together with the preexponential factors $C_{A}^{B}(j)$ describing their weights (coefficient functions). Let us list the resulting expressions for parton distributions 920 .

1. Valence (non-singlet) quark distribution

$$
D^{v a l}(j, \xi)=e^{\nu_{F} \xi} .
$$


2. Sea quarks + antiquarks in a quark:

$$
D_{F}^{s e a}(j, \xi)=\frac{\nu_{F}(j)-\nu_{-}(j)}{\nu_{+}(j)-\nu_{-}(j)} e^{\nu_{+} \xi}+\frac{\nu_{+}(j)-\nu_{F}(j)}{\nu_{+}(j)-\nu_{-}(j)} e^{\nu_{-} \xi}-e^{\nu_{F} \xi}
$$

3. Distribution of a gluon in a quark:

$$
D_{F}^{G}(j, \xi)=\frac{\Phi_{F}^{G}(j)}{\nu_{+}(j)-\nu_{-}(j)}\left(e^{\nu_{+} \xi}-e^{\nu_{-} \xi}\right) .
$$

4. Distribution of quarks + antiquarks in a gluon:

$$
D_{G}^{F}(j, \xi)=\frac{2 n_{f} \Phi_{G}^{F}(j)}{\nu_{+}(j)-\nu_{-}(j)}\left(e^{\nu_{+} \xi}-e^{\nu_{-} \xi}\right) .
$$

5. Gluon distribution in a gluon :

$$
D_{G}^{G}(j, \xi)=\frac{\nu_{+}(j)-\nu_{F}(j)}{\nu_{+}(j)-\nu_{-}(j)} e^{\nu_{+} \xi}+\frac{\nu_{F}(j)-\nu_{-}(j)}{\nu_{+}(j)-\nu_{-}(j)} e^{\nu_{-} \xi} .
$$

Coefficient functions factorize with respect to types of initial and final partons. This can be checked, e.g., by constructing the ratios of $C_{ \pm}$with fixed "signature" \pm

$$
\frac{C_{A(+)}^{F}}{C_{A(+)}^{G}} \quad \text { and } \quad \frac{C_{A(-)}^{F}}{C_{A(-)}^{G}}
$$

which prove to be independent of $A$ - the type of target. This means that one can factorize the coefficient functions entering (1.107)-(1.110) in the seagluon sector into the product of residues determining the coupling of the 
initial parton $A$ and the final parton $B$ to a given trajectory $\nu_{\sigma}$ with signature $\sigma= \pm$ :

$$
D_{A}^{B}(j, \xi)=\sum_{\sigma=+,-} R_{A}(\sigma, j) R^{B}(\sigma, j) e^{\nu_{\sigma}(j) \xi} .
$$

This nice and important property that is to be related to the renormalizability of the theory, reminds one of the factorization property which one meets in Regge theory. There is in fact a close analogy between LLA parton distributions and Regge poles, so that the Regge-terminology, we are using here (i.e. "trajectories", "residues" and "signature"), is not accidential (for more details see Ref. [13]). The residues introduced by (1.112) satisfy the normalization and completeness conditions 66]

$$
\begin{aligned}
\sum_{A=G, F} R_{A}\left(\sigma_{1}, j\right) R^{A}\left(\sigma_{2}, j\right) & =\delta_{\sigma_{1}, \sigma_{2}}, \quad\left(\sigma_{1,2}= \pm\right) \\
\sum_{\sigma= \pm} R_{A}(\sigma, j) R^{B}(\sigma, j) & =\delta_{A}^{B} .
\end{aligned}
$$


Problem 1.1 Derive the "quark" $\rightarrow$ "quark" + scalar "gluon" splitting function (1.37) and reconstruct, with use of the Parton Exchange and Crossing relations (1.88) and (1.89) two remaining kernels. Check, whether there is a Super-Symmetry Relation between fermion and scalar decays.

Answer: $\Phi_{F}^{F}(z)=1-z, \quad \Phi_{F}^{G}(z)=z, \quad \Phi_{G}^{F}(z)=1$.

Problem 1.2 Derive the $F \rightarrow F(z)$ splitting function 1.87a) from a single ladder cell in the axial gauge (1.51).

Solution: The basic structure of the decay cell is

$$
d k_{\perp}^{2} \frac{d \phi}{2 \pi} d \beta \cdot\left(\frac{1-\beta}{k_{\perp}^{2}}\right)^{2} \cdot \frac{1}{(1-\beta)} \cdot \mathcal{M},
$$

where the first factor originates from denominators of virtual propagators

$$
k^{2}=2(p k)=\alpha s=-\frac{k_{\perp}^{2}}{1-\beta},
$$

and the second comes from the $\alpha$-residue of the horizontal ("real") line. The last factor $\mathcal{M}$ stands for the numerator of the amplitude and depends on the process under consideration.

For the case of $F \rightarrow F$ splitting (11.87a) it reads

$$
\mathcal{M}=\frac{1}{2} \operatorname{Tr}\left\{\hat{p} \gamma_{\sigma} \hat{k} \ldots \hat{k} \gamma_{\rho}\right\}\left[-d_{\rho \sigma}(p-k)\right]
$$

After the cyclic permutation of matrices under the Tr we get

$$
\begin{aligned}
\mathcal{M} & =\frac{1}{2} \operatorname{Tr}\left\{\hat{k} \gamma_{\rho} \hat{p} \gamma_{\sigma} \hat{k} \ldots\right\}\left[-g_{\rho \sigma}+\frac{(p-k)_{\rho} q_{\sigma}^{\prime}+q_{\rho}^{\prime}(p-k)_{\sigma}}{(1-\beta) s / 2}\right] \\
& =\frac{1}{2} \operatorname{Tr}\left\{2 \hat{k} \hat{p} \hat{k}-2 k^{2} \frac{\hat{p} \hat{q}^{\prime} \hat{k}+\hat{k} \hat{q}^{\prime} \hat{p}}{(1-\beta) s} \ldots\right\} .
\end{aligned}
$$


To extract the LLA contribution we are allowed to get no more than the square of $k_{\perp}$ from the numerator $\mathcal{M}$ not to loose logarithmic integration over $k_{\perp}^{2}$ in (1.115). Therefore we replace

$$
\hat{k} \hat{p} \hat{k} \approx \hat{k_{\perp}} \hat{p} \hat{k_{\perp}}=k_{\perp}^{2} \cdot \hat{p}, \quad \hat{p} \hat{q}^{\prime} \hat{k}=\hat{k} \hat{q}^{\prime} \hat{p} \approx \beta s \cdot \hat{p}
$$

(the main components $k \approx \beta p$ do not contribute here because of neighboring light-like vectors: $\hat{p} \hat{p}=p^{2}=0$ ). Then, making use of expression (1.116) for the parton virtuality we finally arrive at

$$
\mathcal{M}=k_{\perp}^{2} \cdot\left[2+\frac{4 \beta}{(1-\beta)^{2}}\right] \cdot \frac{1}{2} \operatorname{Tr}\{\hat{p} \ldots\}
$$

which, taken together with (1.115) results in

$$
\frac{d \beta}{\beta} \frac{d k_{\perp}^{2}}{k_{\perp}^{2}} \cdot \frac{1}{2} \operatorname{Tr}\{\beta \hat{p} \ldots\} \cdot\left[2 \frac{1+\beta^{2}}{1-\beta}\right] .
$$

The Tr-structure takes care of the spin-average for the quark $k \approx \beta \hat{p}$, quasi-real and quasi-collinear to initial momentum $p_{\mu}$ with respect to future evolution (upper part of the ladder). The last $\beta$-dependent factor in brackets is nothing but the desired splitting function.

Problem 1.3 Derive the $F \rightarrow G(z)$ splitting function $1.87 b$.

Solution: The main steps of the derivation are the following:

1. In analogy with the previous Problem [1.2, we have for the numerator of the cell 


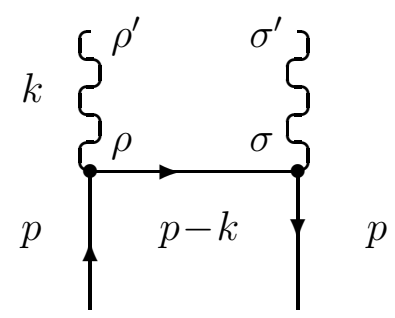

$$
\mathcal{M}=\frac{1}{2} \operatorname{Tr}\left\{\hat{p} \gamma_{\sigma}(\hat{p}-\hat{k}) \gamma_{\rho}\right\} d_{\sigma \sigma^{\prime}}(k) d_{\rho \rho^{\prime}}(k)
$$

2. Neglecting non-logarithmic corrections we estimate

$$
p_{\sigma} \cdot d_{\sigma \sigma^{\prime}}(k)=\frac{k_{\sigma}-\left(k_{\perp}\right)_{\sigma}-\alpha q_{\sigma}^{\prime}}{\beta} d_{\sigma \sigma^{\prime}}(k) \approx-\frac{\left(k_{\perp}\right)_{\sigma}}{\beta}
$$

3. Then the average over quark spin states gives

$$
\frac{1}{2} \operatorname{Tr}\left\{\hat{p} \gamma_{\sigma}(\hat{p}-\hat{k}) \gamma_{\rho}\right\}=4 p_{\sigma} p_{\rho}+2(p k) g_{\sigma \rho}=4 \frac{k_{\perp \sigma} k_{\perp \rho}}{\beta^{2}}-\frac{k_{\perp}^{2}}{1-\beta} \cdot g_{\sigma \rho}
$$

4. Now we are forced to replace gluon polarization state matrices $d(k)$ by $g^{\perp}$ tensors according to (1.53), since the numerator contains already $k_{\perp}^{2}$ to provide us with the transverse log.

5. After averaging over azimuth $\left\langle k_{\perp \sigma} k_{\perp \rho}\right\rangle=-\frac{1}{2} g_{\sigma \rho}^{\perp} \cdot k_{\perp}^{2}$ one comes to the final result:

$$
\frac{d \beta}{\beta} \frac{d k_{\perp}^{2}}{k_{\perp}^{2}} \cdot\left(-\frac{1}{2} g_{\sigma^{\prime} \rho^{\prime}}^{\perp}\right) \cdot\left[2 \frac{1+(1-\beta)^{2}}{\beta}\right]
$$

Notice, that the gluon-parton produced in the LLA decay has two polarizations transverse to the scattering plane $\left(-\frac{1}{2} g_{\sigma^{\prime} \rho^{\prime}}^{\perp}\right.$ stands for the average over gluon spin states). 
Problem 1.4 Derive the $G \rightarrow F(z)$ splitting function 1.87c).

\section{Solution:}

For the amplitude averaged over incoming gluon polarizations one has

$$
\mathcal{M}=\frac{1}{2}\left(-g_{\rho \sigma}^{\perp}\right) \operatorname{Tr}\left\{\hat{k} \gamma_{\rho}(\hat{p}-\hat{k}) \gamma_{\sigma} \hat{k} \ldots\right\}
$$

1. $-\frac{1}{2} g_{\rho \sigma}^{\perp}\left(\gamma_{\rho} \hat{A} \gamma_{\sigma}\right)=-\frac{1}{2}\left(\gamma_{\rho} 2 A_{\perp \rho}-\hat{A} \gamma_{\rho} \gamma_{\rho}\right)=-\hat{A}_{\perp}+\hat{A}=\hat{A}_{\|}$

2. $\mathcal{M}=\hat{k}\left[(1-\beta) \hat{p}-\alpha \hat{q}^{\prime}\right] \hat{k}=(1-\beta) k_{\perp}^{2} \cdot \hat{p}+\frac{k_{\perp}^{2}}{(1-\beta) s} \beta^{2} \cdot \hat{p} \hat{q}^{\prime} \hat{p}$

3. The whole expression for the ladder cell (1.115) now reads

$$
\frac{d \beta}{\beta} \frac{d k_{\perp}^{2}}{k_{\perp}^{2}} \cdot \frac{1}{2} \operatorname{Tr}\{\beta \hat{p} \ldots\} \cdot\left[2\left(\beta^{2}+(1-\beta)^{2}\right)\right]
$$

Problem 1.5 From the LLA parton distribution (1.107)-(1.110) derive explicit expressions for the residues $R_{A}(\sigma)$ and $R^{B}(\sigma)$ defined by (1.112)

Answer:

$$
\begin{gathered}
R_{G}(\sigma, j)=R^{G}(\sigma, j)=\left[\nu_{\sigma}(j)-\nu_{0}(j)\right] C_{\sigma}^{-1 / 2}(j) ; \\
R_{F}(\sigma, j)=\Phi_{F}^{G}(j) C_{\sigma}^{-1 / 2}(j), \quad R^{F}(\sigma, j)=\Phi_{G}^{F}(j) C_{\sigma}^{-1 / 2}(j) ; \\
\text { with } \quad C_{\sigma}(j)=\left[\nu_{+}(j)-\nu_{-}(j)\right] \cdot\left|\nu_{\sigma}(j)-\nu_{0}(j)\right| .
\end{gathered}
$$




\section{Bibliography}

1. R.P. Feynman. Photon-hadron interaction. Benjamin, New York, 1972.

2. V.N. Gribov. In Proceedings of the XVII Winter School of the LNPI, Leningrad.

3. V.N. Gribov. Lund University preprint LU TP 91-07, 1991.

4. V.N. Gribov and L.N. Lipatov. Sov. J. Nucl. Phys., 15: 438 and 675, 1972.

5. L.N. Lipatov. Sov. J. Nucl. Phys., 20: 94, 1975.

6. A.P. Bukhvostov, L.N. Lipatov and N.P. Popov. Sov. J. Nucl. Phys., 20: 287, 1975.

7. V.N. Baier, V.S. Fadin and V.A. Khoze. Nucl. Phys., B65: 381, 1973.

8. G. Altarelli and G. Parisi. Nucl. Phys., B126: 298, 1977.

9. Yu.L. Dokshitzer. Sov. Phys. JETP, 46: 641, 1977.

10. N. Christ, B. Hasslacher and A.H. Mueller. Phys. Rev., D6: 3543, 1972.

11. D. Gross and F. Wilczeck. Phys. Rev., D8: 3633, 1973. ibid, D9: 980, 1974.

H. Georgi and H.D. Politzer. Phys. Rev., D9: 416, 1974.

12. A.P. Bukhvostov, G.V. Frolov, L.N. Lipatov and E.A. Kuraev. Nucl. Phys., B258:601, 1985.

13. Yu.L. Dokshitzer, D.I. Dyakonov and S.I. Troyan. Phys. Rep., 58: 270, 1980.

14. A.H. Mueller. Phys. Rep., 73: 237, 1981.

15. G. Altarelli. Phys. Rep., 81: 1, 1982.

16. B.L. Ioffe, V.A. Khoze and L.N. Lipatov. Hard Processes. North Holland, 1984. 
17. T. Kinoshita. Journ. Math. Phys., 3: 650, 1962.

T.D. Lee and M. Nauenberg. Phys. Rev., 113: 1549, 1964.

18. H.D. Politzer. Phys. Rep., 14C: 130, 1974.

19. J.B. Gaffney and A.H. Mueller. Nucl. Phys., B250: 109, 1985.

20. A.J. Buras. Nucl. Phys., B126: 125, 1977. 


\section{Chapter 2}

\section{Spin Dependent Deep Inelastic Lepton Scattering and the Role of the Axial Anomaly}

\section{Contents}

2.1 The Box Graph, the Triangle Graph and the OPE 66

2.1 .1 The Box Graph . . . . . . . . . . . . . . . . . . 67

2.1 .2 The Triangle Graph . . . . . . . . . . . . . . . . 73

2.1 .3 The Operator Product Expansion . . . . . . . . 75

2.2 Partonic Interpretation of Spin Dependent DIS . 75

2.2.1 Phenomenological Definitions of Quarks and Gluons 76

2.2.2 Light-Cone Wave Function Definition of Partons . 78

$2.3 \quad$ Parton and Constituent Quark Model of Hadrons 81 
2.4 The Experimental Situation . . . . . . . . . . 85

Bibliography . . . . . . . . . . . . . . 88

The parton model was developed primarily in order to explain the results found in spin independent deep inelastic electron scattering.

The new element which we wish to focus on in this Chapter is the fact that due to the axial anomaly 112 the relationship between matrix elements of local gauge invariant quark operators and moments of quark parton distributions is partially lost. We shall explain in some detail how the matrix elements of the flavor singlet axial vector current are determined not solely by the first moment of a quark distribution but also, partly, by the first moment of a spin-dependent gluon distribution.

\subsection{The Box Graph, the Triangle Graph and the Operator Product Expansion}

In this section we shall consider the essentially new element which appears in spin dependent DIS 3-7 as compared to spin independent scattering. We begin our discussion with an analysis of the fermion box graph contribution to the structure function $g_{1}$ defined by (1.28b).

In our discussion we shall find it convenient to use a light-cone momentum notation where a vector

$$
v_{\mu}=\left(v_{-}, v_{+}, v_{\perp}\right) \quad \text { with } \quad v_{ \pm}=\frac{v_{0} \pm v_{3}}{\sqrt{2}} .
$$

Then for the DIS process we choose a frame where $p_{\mu}=\left(p_{-}, p_{+}, 0,0\right)$ and $q_{\mu}=\left(q_{-}, q_{+}, 0,0\right)$ and with $x=-q^{2} / 2 q \cdot p=-q_{+} / p_{+}\left(1+O\left(M^{2} / Q^{2}\right)\right)$. That 
is we choose $p_{+} q_{-} \gg p_{-} q_{+}$. Then we may extract $g_{1}$ from the decomposition (1.23) for $W_{\mu \nu}(s)$ by considering

$$
\sum_{i, j=1}^{2} \epsilon_{i j} W_{i j}(s) \quad \text { where } \quad \epsilon_{12}=1=-\epsilon_{21} \quad \text { and } \quad \epsilon_{11}=\epsilon_{22}=0 .
$$

Then, using (1.23 1.25$)$ and the relation $s_{ \pm}= \pm\left(s_{3} / M\right) p_{ \pm}$for $\vec{s}=s_{3} \vec{e}_{3}$, one finds

$$
\frac{i}{2 \pi} \sum_{i, j=1}^{2} \epsilon_{i j} W_{i j}(s)=s_{3} \frac{\nu}{M^{2}} G_{1}=g_{1} s_{3} .
$$

(2.1) is the equation we shall use in order to extract $g_{1}$ in the calculations to be carried out in the following sections of this Chapter.

\subsubsection{The Box Graph}

Consider the contribution to $W_{\mu \nu}(s)$ of the graph illustrated in Fig. 2.1] where the wiggly internal lines are gluons, the line $q$ is a virtual photon having vector indices $i, j=1,2$ and where the photons and gluons connect to a quark loop, the quark having mass $m$. We work in light-cone gauge where the propagator of the $l$-line on the left hand side of the graph of Fig. 2.1] is

$$
D_{\alpha \alpha^{\prime}}(l)=\frac{-i}{l^{2}}\left[g_{\alpha \alpha^{\prime}}-\frac{l_{\alpha} \eta_{\alpha^{\prime}}+l_{\alpha^{\prime}} \eta_{\alpha}}{\eta \cdot l}\right]
$$

with $\eta \cdot v=v_{+}$for any four-vector $v$. It is convenient to write

$$
-\left(g_{\alpha \alpha^{\prime}}-\frac{l_{\alpha} \eta_{\alpha^{\prime}}+l_{\alpha^{\prime}} \eta_{\alpha}}{\eta \cdot l}\right)=-\left(g_{\alpha \alpha^{\prime}}-\frac{\bar{l}_{\alpha} \eta_{\alpha^{\prime}}+\bar{l}_{\alpha^{\prime}} \eta_{\alpha}}{\eta \cdot l}\right)+\frac{\eta_{\alpha} \eta_{\alpha^{\prime}} l^{2}}{(\eta \cdot l)^{2}}
$$



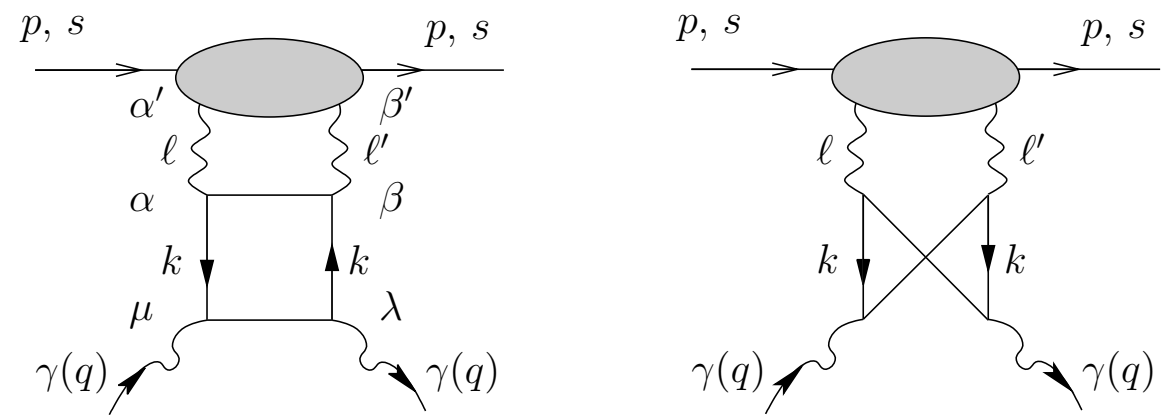

Figure 2.1: Box graph contributions to deep inelastic scattering.

where $l_{\alpha}=\bar{l}_{\alpha}+\frac{l^{2}}{2 \eta l} \eta_{\alpha}$ and $\bar{l}$ is a light-like momentum.

So long as $l_{\perp}^{2} / Q^{2} \ll 1$ and $l^{2} / Q^{2} \ll 1$ we may drop the final term on the right hand side of (2.3) and, in evaluating the fermion loop part of the graph of Fig. 2.1 we may take $l_{\perp}=0$ for fixed $l_{+}$and fixed $l^{2}$. (The argument for the assertion made goes as follows: According to (2.1) we always understand the projection $\epsilon_{i j}$ on the photon indices of the fermion loop shown in Fig. 2.1. Then for the first of the two graphs in that figure we replace $\gamma_{j} \gamma \cdot(q+k) \gamma_{i} \epsilon_{i j}$ by $q_{-} \epsilon_{i j} \gamma_{j} \gamma_{+} \gamma_{i}=-2 i q_{-} \gamma_{+} \gamma_{5}$ a replacement clearly valid when $\left|k^{2}\right| \ll Q^{2}$, the case when $\left|k^{2}\right| \sim Q^{2}$ to be considered later. But the trace in the fermion loop now is $\operatorname{Tr} \gamma_{+} \gamma_{5} \gamma \cdot k \gamma_{\alpha} \gamma \cdot(k-l) \gamma_{\beta} \gamma \cdot k$ which, after performing the loop integral over $k$, can only lead to a tensor structure $\epsilon_{+\alpha \beta \lambda} l_{\lambda}$. But for this tensor structure it is easy to check that only transverse components of $\alpha \beta$ are effective when multiplied by the propagator given in (2.3) and that there is no deApendence on $l_{\perp}$.

Now it remains to consider the remaining part of $B \gamma_{j} \gamma \cdot(q+k) \gamma_{i} \epsilon_{i j}$ along with the second graph, the crossed box graph, of Fig. 2.1. But here the loop momentum $k_{\perp}$ must be on the order of $Q$ in order to get a contribution which 
is not higher twist. But when $k_{\perp} \sim Q$ we clearly may set $l_{\perp}=0$, for fixed $l^{2}$ and fixed $l_{+}$, and we may clearly neglect the $\eta_{\alpha} \eta_{\alpha^{\prime}} l^{2} /(\eta \cdot l)^{2}$ term in (2.3) since the $l^{2}$ must appear in the final form $l^{2} / Q^{2}$ or $l^{2} /(q \cdot l)$, terms of higher twist.)

Thus instead of the propagator (2.2) we may use

$$
\bar{D}_{\alpha \alpha^{\prime}}(l)=\frac{-i}{l^{2}+i \epsilon} \sum_{\lambda= \pm} \epsilon_{\alpha}^{\lambda}(\bar{l}) \epsilon_{\alpha^{\prime}}^{\lambda}(\bar{l})
$$

where $\epsilon_{\alpha}^{\lambda}(\bar{l})$ obeys

$$
\bar{l} \cdot \epsilon^{\lambda}=\eta \cdot \epsilon^{\lambda}=0
$$

the conditions for physical polarizations in light-cone quantization in lightcone gauge. Using (2.5) one can write $\epsilon_{\alpha}^{\lambda}$ more explicitely as

$$
\epsilon_{\alpha}^{\lambda}(\bar{l})=\left(\epsilon_{-}^{\lambda}, \epsilon_{+}^{\lambda}, \epsilon_{\perp}^{\lambda}\right)=\left(\frac{l_{\perp} \cdot \epsilon_{\perp}^{\lambda}}{\eta \cdot l}, 0, \epsilon_{\perp}^{\lambda}\right)
$$

and we note that $l \cdot \epsilon_{\alpha}^{\lambda}(\bar{l})=0$.

In order to extract $g_{1}$ we use (2.1). Note that

$$
-i \epsilon_{i j}=\epsilon_{i}^{+}(q) \epsilon_{j}^{+}(q)^{*}-\epsilon_{i}^{-}(q) \epsilon_{j}^{-}(q)^{*}
$$

so that the $\epsilon_{i j}$ projection in (2.1) represents the difference of $(+)-(-)$ helicity amplitudes for the virtual photon. Thus we may write

$$
g_{1}\left(x, Q^{2}\right)=\frac{\left\langle e^{2}\right\rangle}{2} \int \frac{d^{4} l}{(2 \pi)^{4}} B(p, l) A(l, q) . B
$$

$A$ and $B$ are illustrated in Fig. 2.2 and are given by the expressions

$$
A=\frac{1}{2 \pi} \frac{2 T N_{f} g_{s}^{2}}{(2 \pi)^{4}} \int d^{4} k \frac{2 \pi \delta\left((k-l)^{2}-m^{2}\right) 2 \pi \delta\left((q+k)^{2}-m^{2}\right)}{\left[k^{2}-m^{2}\right]^{2}} \operatorname{Tr}\{\}
$$



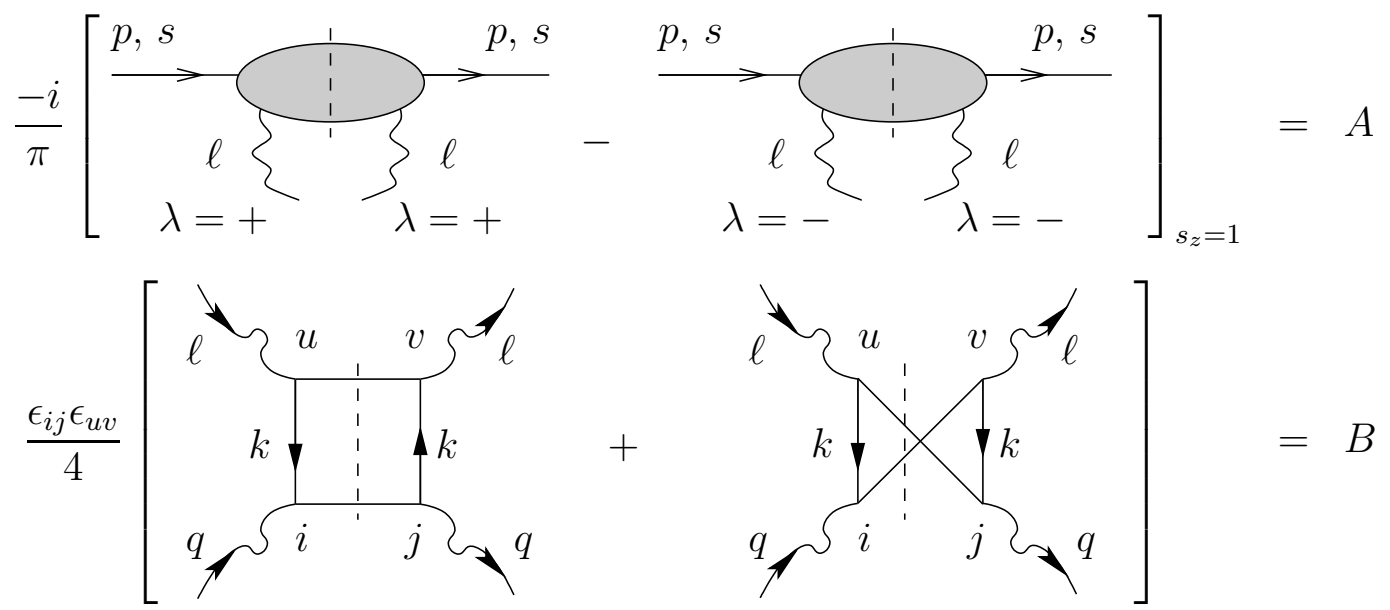

Figure 2.2: Pictorial definitions of $A$ and $B$ as given in (2.9) and (2.11).

with

$$
\operatorname{Tr}\{\}=\frac{\epsilon_{i j} \epsilon_{u v}}{4} \operatorname{Tr}\left\{\gamma_{v}[\gamma \cdot k+m] \gamma_{j}[\gamma \cdot(q+k)+m] \gamma_{i}[\gamma \cdot k+m] \gamma_{u}[\gamma \cdot(l-k)+m]\right\}
$$

and

$$
\begin{aligned}
& B(p, l)=\sum_{a} \int d^{4} x e^{i l x} \\
& \left.\cdot\left\langle p s\left|\left[A^{a}(x) \cdot \epsilon^{+}(\bar{l}) A^{a}(0) \cdot \epsilon^{+}(\bar{l})^{*}-A^{a}(x) \cdot \epsilon^{-}(\bar{l}) A^{a}(0) \cdot \epsilon^{-}(\bar{l})^{*}\right]\right| p s\right\rangle\right|_{\vec{s}=\vec{e}_{z}}
\end{aligned}
$$

with $a$ the color index of the gluon field $A_{\mu}^{a}(x)$. For $A$ we have only written down the contribution of the first graph on the left hand side of the equation 
illustrated in Fig. 2.2 b. The $\epsilon_{u v}$ in (2.10) comes from using an equation identical to (2.7) but for the $\left(\epsilon_{\perp}^{\lambda}\right)_{i}$ 's. Only diagonal gluon polarization elements appear in (2.11) because a forward matrix element is being considered. Also $\left\langle e^{2}\right\rangle$ in (2.8) is the average electric charge squared for the quark loops appearing in Fig. [2.2 b. Finally, as discussed earlier we may take $l_{\perp}=0$, for fixed $l_{+}$ and for fixed $l^{2}$, in (2.9) and (2.10), but $l_{\perp}$ cannot be set to zero in (2.11). The resulting $d^{2} l_{\perp}$ integral in (2.8) will be logarithmically divergent so that a cutoff, $\vartheta\left(Q^{2}-l_{\perp}^{2}\right)$, must be understood. This cutoff is what will give the $Q^{2}$ dependence of the polarized gluon distribution at leading logarithmic level.

Now it is straightforward to evaluate $A$ as

$$
A=-\frac{\alpha_{s} N_{f}}{2 \pi} \int_{0}^{K^{2}} \frac{d k_{\perp}^{2}}{\sqrt{1-k_{\perp}^{2} / K^{2}}}\left(\frac{(1-2 z)\left(k_{\perp}^{2}+m^{2}\right)-2 m^{2}(1-z)}{\left[k_{\perp}^{2}+m^{2}+L^{2} z(1-z)\right]^{2}}-\frac{2 z(1-2 z)}{Q^{2}(1-z)}\right)
$$

with $L^{2}=-l^{2}, K^{2}=[(1-x) / 4 x] Q^{2}$ and $z=Q^{2} / 2(q \cdot l)$. Writing

$$
d^{4} l=\frac{\pi}{2} d l_{\perp}^{2} d L^{2} \frac{d z}{z}
$$

one finds

$$
g_{1}\left(x, Q^{2}\right)=\frac{\left\langle e^{2}\right\rangle}{2} \int \frac{d l_{\perp}^{2} d L^{2} d z}{32 \pi^{3} z} B\left(L^{2}, d l_{\perp}^{2}, x / z\right) A\left(L^{2}, z, Q^{2}\right) \vartheta\left(Q^{2}-l_{\perp}^{2}\right)
$$

The spin dependent gluon distribution for the nucleon is given in terms of $B$ by

$$
\Delta g\left(x, Q^{2}\right)=\int \vartheta\left(Q^{2}-l_{\perp}^{2}\right) \frac{d l_{\perp}^{2} d L^{2}}{32 \pi^{3}} B\left(L^{2}, d l_{\perp}^{2}, x\right)
$$

where our normalization is such that $\Delta g\left(Q^{2}\right)=\int_{0}^{1} d x \Delta g\left(x, Q^{2}\right)$ represents the amount of helicity carried by gluons in the nucleon. In what follows we 
shall be mainly concerned with

$$
M_{1}\left(Q^{2}\right)=\int_{0}^{1} d x g_{1}\left(x, Q^{2}\right),
$$

the first moment of $g_{1}$.

In order to understand, and interpret, spin dependent DIS it is important to investigate in some detail the final states contributing to (2.15). To that end consider

$$
A_{1}\left(k_{\perp}^{2}\right)=\int_{0}^{1} d z A\left(k_{\perp}^{2}, z, Q^{2}\right)
$$

where $A\left(k_{\perp}^{2}, z, Q^{2}\right)$ is the integrand on the right hand side of (2.12). It is straightforward to see from (2.12) that when $m^{2} / k_{\perp}^{2}, L^{2} / k_{\perp}^{2}, k_{\perp}{ }^{2} / Q^{2} \ll 1$ then $A_{1}\left(k_{\perp}^{2}\right)$ is small. Also one can see from (2.12) that if $m^{2} / L^{2} \ll 1$ then $A_{1}$ is small if $k_{\perp}{ }^{2} / Q^{2} \ll 1$. Finally if $L^{2} / m^{2} \ll 1, \int d k_{\perp}^{2} A_{1}\left(k_{\perp}^{2}\right)=0$ which result will become clear from our discussion of the triangle graph a little farther on.

Thus when one considers the first moment of the deep inelastic photongluon spin dependent cross section the fermion loop gives one contribution at $k_{\perp} \sim Q$ and another contribution at $k_{\perp} \sim m$, so long as $m^{2} / L^{2}$ is not too small. The region $m^{2} \ll k_{\perp}^{2} \ll Q^{2}$, the usual logarithmic domain of DIS does not contribute here.

If we break up the $d k_{\perp}^{2}$ integral in (2.12) into a (low) region $0 \leq k_{\perp}^{2} \leq$ $(1 / \epsilon) m^{2}$ and a (high) region $\epsilon Q^{2} B \leq k_{\perp}^{2}<\infty$ for a fixed small $\epsilon$ then we may verify that

$$
\int_{0}^{1} d x A^{h i g h}=A_{1}^{h i g h}=-\frac{\alpha_{s} N_{f}}{2 \pi} .
$$


Now taking the first moment one gets

$$
\frac{2}{\left\langle e^{2}\right\rangle} M_{1}\left(Q^{2}\right)=\int \frac{d l_{\perp}^{2} d L^{2}}{32 \pi^{3}} B_{1}\left(L^{2}, l_{\perp}^{2}\right) A_{1}^{l o w}\left(L^{2}\right) \vartheta\left(Q^{2}-l_{\perp}^{2}\right)-\frac{\alpha_{s} N_{f}}{2 \pi} \Delta g\left(Q^{2}\right) .
$$

\subsubsection{The Triangle Graph}

In order to check the operator product expansion 8 and to understand the role of the axial anomaly it is important to investigate the triangle graph contribution to

$$
\frac{1}{2 p_{+}} \Gamma_{5 \mu}=\left.\left\langle p s\left|j_{5 \mu}(0)\right| p s\right\rangle\right|_{\vec{s}_{\vec{B}} \vec{e}_{z}}
$$

with $j_{5 \mu}=\sum_{f} \tilde{q}_{f} \gamma_{\mu} \gamma_{5} q_{f}$ the flavor singlet axial vector current. The relevant graph is the one illustrated in Fig. 2.3. As in (2.8) we may write

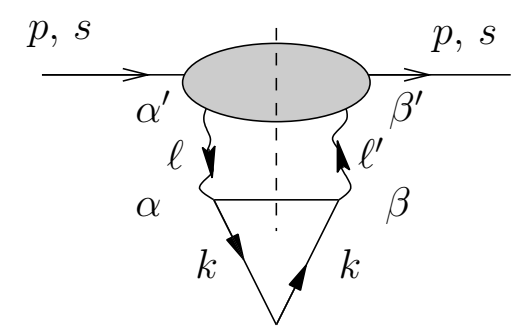

Figure 2.3: Kinematics of the triangle graph.

$$
\frac{1}{2 p_{+}} \Gamma_{5 \mu}=\int \frac{d l_{\perp}^{2} d L^{2} d x}{32 \pi^{3}} B\left(L^{2}, l_{\perp}^{2}, x\right) \frac{1}{2 l_{+}} \Gamma_{5+}^{g} \vartheta\left(Q^{2}-l_{\perp}^{2}\right)
$$


where $z=l_{+} / p_{+}$and where

$$
\Gamma_{5+}^{g}=\lim _{n \rightarrow 4} \frac{2 T N_{f} g^{2}}{(2 \pi)^{4}} \int d^{n-2} k_{\perp} d k_{+} d k_{-} \frac{2 \pi \delta\left((l-k)^{2}-m^{2}\right)}{\left[k^{2}-m^{2}\right]^{2}} \operatorname{Tr}\{\}
$$

with

$$
\operatorname{Tr}\{\}=\operatorname{Tr}\left\{\gamma \cdot \epsilon^{*}[\gamma \cdot k+m] \gamma_{+} \gamma_{5}[\gamma \cdot k+m] \gamma \cdot \epsilon[\gamma \cdot(l-k)-m]\right\}
$$

We have continued the transverse integrations to $n-2$ dimensions for the gluonic matrix element of the axial vector current given in (2.21) and we have taken $\gamma_{5}=i \gamma_{0} \gamma_{1} \gamma_{2} \gamma_{3}$ which choice of $\gamma_{5}$ is known to give the correct axial anomaly when using dimensional regularization 910. In (2.22) $\epsilon=$ $\frac{1}{\sqrt{2}}(0,0,1, i)$.

The trace in (2.22) is straightforward to do. One finds

$$
\begin{aligned}
& \frac{1}{2 l_{+}} \Gamma_{5+}^{g}=\lim _{n \rightarrow 4}-\frac{\alpha_{s} N_{f}}{2 \pi^{2}} \int d z \int d^{n-2} k_{\perp} \\
& \cdot \frac{\left(k_{\perp}^{2}+m^{2}\right)(1-2 z)-2 m^{2}(1-z)-2 \frac{n-4}{n-2} k_{\perp}^{2}(1-x)}{\left[k_{\perp}^{2}+m^{2}+L^{2} z(1-z)\right]^{2}}
\end{aligned}
$$

The $n-4$ term arises because $\gamma_{5}$ is not Lorentz invariant in $n$ dimensions. This produces a term which is proportional to $\hat{k}^{2}$ where $\hat{k}$ is the projection of $k$ onto the $n-4$ regulator dimensions. After an azimuth average $\hat{k}^{2}=k_{\perp}^{2} \frac{n-4}{n-2}$. One finds $[5]$

$$
\frac{1}{2 l_{+}} \Gamma_{5}^{g}=-\frac{\alpha_{s} N_{f}}{2 \pi}\left(1-\int_{0}^{1} \frac{2 m^{2}(1-z) d z}{m^{2}+L^{2} z(1-z)}\right) .
$$


It is easy to check that

$$
\begin{aligned}
\lim _{m^{2} / L^{2} \rightarrow 0} \frac{1}{2 l_{+}} \Gamma_{5}^{g} & =-\frac{\alpha_{s} N_{f}}{2 \pi} \\
\lim _{m^{2} / L^{2} \rightarrow \infty} \frac{1}{2 l_{+}} \Gamma_{5}^{g} & =0 .
\end{aligned}
$$

\subsubsection{The Operator Product Expansion}

Taking the first moment of $g_{1}\left(x, Q^{2}\right)$, as given by (2.18) and comparing the result with (2.20) one sees that the operator product expansion will be valid so long as the first moment of $A$, as given by (2.12) agrees with $\frac{1}{2 l_{+}} \Gamma_{5+}^{g}$, as given by (2.23). The low $k_{\perp}^{2}$ regions of (2.12) and (2.23) are clearly the same once the first moment of $A$ is taken. As for the high $k_{\perp}^{2}$ regions both the first

moment of $A$ and $\frac{1}{2 l_{+}} \Gamma_{5+}^{g}$ have the value $-\frac{\alpha_{s} N_{f}}{2 \pi}$ as evaluated in (2.17) and (2.25a). Thus the operator product expansion works and serves to connect the first moment of the graphs shown in Fig. 2.1 with the graph given in Fig. 2.3.

\subsection{The Partonic Interpretation of Spin Dependent Deep Inelastic Scattering}

In this section we shall interpret the results found in the previous section. We shall restrict ourselves to the fermion box and triangle graphs, connected by gluons to the nucleon, shown in Fig. 2.1 and 2.3 since it is only for these graphs that any subtlety arises in the partonic interpretation. 


\subsubsection{Phenomenological Definitions of Quarks and Gluons}

It is important to have a clear understanding of what exactly is meant by a partonic (quark or gluon) component of a hadron, in order to be able to identify correctly the amount of spin carried by the various partons. There are two ways to do this. The first way, a phenomenological procedure 517 , is to identify the final state jets produced in a hard reaction. From the final state jets one infers a hard scattering subprocess of which the initial states are either leptons or partonic components of an initial state hadron. In the case of deep inelastic lepton-nucleon scattering we will reconstruct the hard scattering from observations of the outgoing lepton and of the jets produced at large transverse momentum.

1. Suppose that one and only one jet is produced at large transverse momentum, as illustrated in Fig. 2.4.

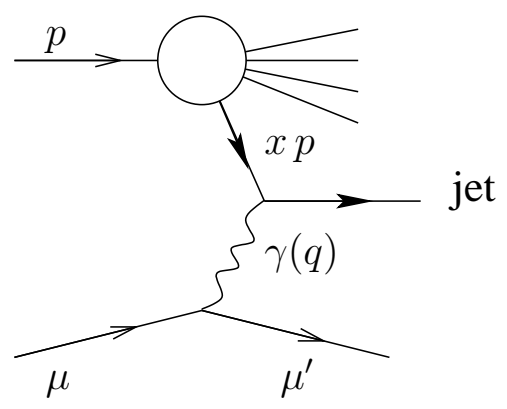

Figure 2.4: Single jet production in deep inelastic scattering.

(In addition there is always a beam jet at low transverse momentum.) In QCD such events can only come from the subprocess $\gamma(q)+$ quark $(x p) \rightarrow$ 
quark jet $(q+x p)$. By measuring the momentum of the jet and the probability of production of such jets one determines the number density of quarks, having longitudinal momentum fraction $x$, in the initial nucleon. In terms of our discussion in the previous section these one jet events correspond to the region of low $k_{\perp}$ in Fig. 2.1. Such events are contained in the first term on the right hand side of (2.18), after integrating over all $x$-values of the produced jets.

2. Suppose one measures events with two distinct high transverse momentum jets as illustrated in Fig. 2.5 where two quark jets are produced.

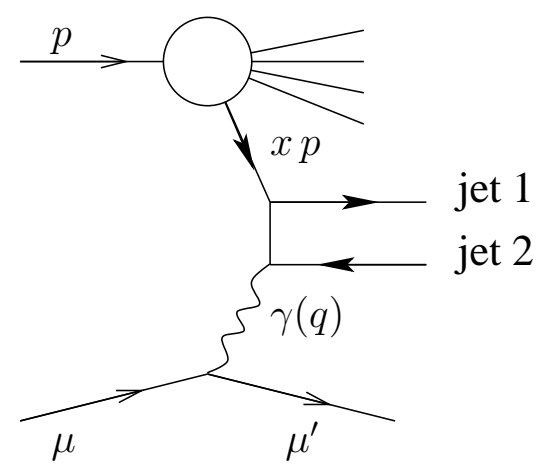

Figure 2.5: Two jet production in deep inelastic scattering.

Here one infers the hard subprocess $\gamma(q)+$ gluon $(x p) \rightarrow$ jet $1+$ jet 2 and so the two jet process can be used as a method of determining the gluon distribution of the nucleon. (In general 2-jet production in DIS may consist of two jets, coming from a gluon distribution in the nucleon, or of a quark jet and a gluon jet coming from a quark distribution in 
the nucleon. In the present spin dependent scattering the two quark jet production dominates by a power of $\alpha_{s}\left(Q^{2}\right)$, after the first moment in $x$ is taken.) In terms of our earlier discussion the two jet events correspond to the region $k_{\perp} \sim Q$ in Fig. 2.1] and such events are included in the second term on the right hand side of (2.18), the $-\frac{\alpha_{s} N_{f}}{2 \pi} \Delta g\left(Q^{2}\right)$ term.

Thus we reach the surprising conclusion that a part of the first moment of $g_{1}\left(x, Q^{2}\right)$, the second term on the right hand side of (2.18), should be identical with a gluonic parton distribution. This is true even though the operator product expansion gives this contribution in terms of the high- $k_{\perp}$ components of the matrix elements of the fermionic operator $j_{5 \mu}$, that is the high- $k_{\perp}$ part of (2.23) given by (2.25a ). It is important to realize the high- $k_{\perp}$ part of (2.18) corresponds to the final term in the integrand in (2.23), a term which exists only because we must go outside of physical four-dimensional space-time in order to define matrix elements of $j_{5 \mu}$.

\subsubsection{Light-Cone Wave Function Definition of Partons}

Intuitively partons are supposed to be the objects, measured over short times and over short distances, that make up hadrons. This is given a precise formulation by writing, say, the proton's wavefunction, in light-cone quantization and in light-cone gauge, in terms of Fock space components consisting of gluons, quarks and anti-quarks with renormalization being carried out at some scale $\mu$ which is much greater than the fundamental QCD scale $\Lambda$ 피 The quark and gluon number densities are then just the expectations of the 
number density operator in light-cone quantization. For example

$$
\begin{aligned}
x G\left(x, Q^{2}\right)= & \left\langle\int d^{2} k_{\perp} \vartheta\left(Q^{2}-k_{\perp}^{2}\right) d k_{+} x \delta\left(x-\frac{k_{+}}{p_{+}}\right) \sum_{\lambda= \pm} a_{\lambda}^{+}\left(k_{\perp}, k_{+}\right) a_{\lambda}\left(k_{\perp}, k_{+}\right)\right\rangle
\end{aligned}
$$

with the \langle\rangle referring to the expectation in the state of a proton having momentum $p$ so long as $p_{\perp}^{2} \ll Q^{2}$. $a_{\lambda}$ refers to the annihilation operator of a gluon having polarization $\lambda$ with color indices being suppressed in (2.26)).

Now let us try to understand (2.23) in terms of a quark-anti-quark pair arising from a virtual gluon in the proton, an interpretation suggested by (2.14) and (2.15). To that end consider

$$
\begin{aligned}
\frac{1}{2 l_{+}} \tilde{\Gamma}_{5}^{g}= & T N_{f} \sum_{r, r^{\prime}} \int d^{2} k_{\perp} \int_{0}^{l_{+}} d k_{+}\left|\psi_{r, r^{\prime}}(l-k, k)\right|^{2} \\
& \left(\frac{\tilde{u}_{r}(k) \gamma_{+} \gamma_{5} u_{r}(k)}{2 k_{+}}+\frac{\tilde{v}_{r^{\prime}}(l-k) \gamma_{+} \gamma_{5} v_{r^{\prime}}(l-k)}{2(l-k)_{+}}\right)
\end{aligned}
$$

with the light-cone wavefunction for the quark-anti-quark pair in the virtual gluon given as

$$
\psi_{r, r^{\prime}}(l-k, k)=g \frac{\tilde{u}_{r}(k) \gamma \cdot \epsilon v_{r^{\prime}}(l-k)}{\sqrt{(2 \pi)^{3} 2 k_{+} 2(l-k)_{+}}\left[l_{-}-\frac{k_{\perp}^{2}+m^{2}}{2 k_{+}}-\frac{k_{\perp}^{2}+m^{2}}{2(l-k)_{+}}\right]} .
$$

Now we can check that (2.28) agrees with (2.23) if one drops the $\frac{n-4}{n-2}$ factor in the numerator of (2.23). In particular for $m=0$

$$
\tilde{u}_{r}(k) \gamma \cdot \epsilon v_{r^{\prime}}(l-k)=\frac{k_{1}+i k_{2}}{\sqrt{2 z(1-z)}} \delta_{r r^{\prime}}[r(1-2 x)-1]
$$


while

$$
\tilde{u}_{r}(k) \gamma_{+} \gamma_{5} u_{r}(k)=\tilde{v}_{r}(k) \gamma_{+} \gamma_{5} v_{r}(k)=2 r k_{+}
$$

with $r= \pm 1$ and $r=+1$ corresponding to positive helicity for a quark and negative helicity for an anti-quark. It is straightforward to see that (2.28)(2.30) used in (2.27) give the zero mass limit of (2.23) when the $\frac{n-4}{n-2}$ factor is dropped in the integrand of (2.23). The $\delta_{r r^{\prime}}$ in (2.29) requires that the quark and anti-quark coming from the virtual photon carry opposite helicity if the quarks have zero mass.

Thus one can obtain the low- $k_{\perp}$ part of $\Gamma_{5+}^{g}$ in terms of a Fock space calculation of quark-anti-quark pair production. Referring to (2.18) it is clear that by using a light-cone wavefunction to calculate the first moment of $g_{1}$ we will correctly obtain the first term on the right hand side of that equation but the second term, the $-\frac{\alpha_{s} N_{f}}{2 \pi} \Delta g$, will be missing. We are thus led to write

$$
\frac{2}{\left\langle e^{2}\right\rangle} M_{1}\left(Q^{2}\right)=\sum_{f}\left(\frac{e_{f}^{2}}{\left\langle e^{2}\right\rangle} \Delta q_{f}\left(Q^{2}\right)-\frac{\alpha_{s}\left(Q^{2}\right)}{2 \pi} \Delta g\left(Q^{2}\right)\right)
$$

with the two terms on the right hand side of (2.31) corresponding to the two terms on the right hand side of (2.18) and where $f$ should be summed over all flavors for which $m_{f}^{2} / Q^{2} \ll 1$. In order to obtain the $-\frac{\alpha_{s} N_{f}}{2 \pi} \Delta g$ term in light-cone quantization we may do a light-cone wavefunction calculation using $(n-2)$ transverse dimensions and then let $n \rightarrow 4$. Such a calculation reproduces (2.23) and hence (2.18) and (2.31) exactly.

In (2.31) $\Delta q_{f}$ clearly represents the fraction of the proton's spin carried by quarks and anti-quarks of flavor $f$. However, the $-\frac{\alpha_{s}}{2 \pi}$ factor in (2.31) resides in the $\hat{k} \rightarrow \infty$ region of the quark Fock space, where $\hat{k}$ is the projection of $k$ onto the $n-4$ unphysical degrees of freedom. Thus it is difficult to associate the $-\frac{\alpha_{s} N_{f}}{2 \pi}$ with physical degrees of freedom. 
However, since $\hat{k} \rightarrow \infty$ means that the quark loop has shrunk to a point (The physical $k_{\perp} \rightarrow \infty$ in this limit also.) it becomes natural to associate the $-\frac{\alpha_{s} N_{f}}{2 \pi}$ directly with the gluonic component of the Fock space. Thus we are led to identify the $-\frac{\alpha_{s} N_{f}}{2 \pi} \Delta g\left(Q^{2}\right)$ contribution in (2.18) and (2.31) as a gluonic component in agreement with the definition of partonic components in terms of jets discussed previously.

\subsection{Relationship Between the Parton Description of a Hadron and the Constituent Quark Description}

In our previous discussion we have described a nucleon as being composed of quarks and gluons. These quark and gluon distributions carry a momentum scale describing how locally one measures the partons in the nucleon. Thus $q_{f}\left(x, Q^{2}\right)$ is the number density of quarks of flavor $f$ having a longitudinal momentum fraction $x$ and whose transverse size is $1 / Q$. In the light-cone quantization formalizm the scale $Q$ enters as the scale at which renormalization is done. In an experimental situation $1 / Q$ is the resolution, in transverse coordinate space, of the measurer of the quark or gluon.

There is also another picture of the nucleon to which we have become accustomed and which has been extremely important for our understanding of the spectrum of low lying hadrons. This picture is, of course, the constituent quark model of the nucleon.

In the constituent quark model one pictures the proton as made of two up quarks and a down quark with the spin of the proton being given by the resulting spin of the three quarks in their ground state wavefunction. We shall consider here only the simplest of the constituent quark models in which the 
constituent up and down quarks have a mass equal to one third the nucleon mass with binding energy being neglected completely. At first sight the parton picture of the proton and the constituent quark picture appear at odds. The constituent quark picture has no room for qluonic degrees of freedom while one knows from studies of deep inelastic lepton-nucleon scattering that gluons carry about one-half of the longitudinal momentum of a fast proton.

In order to sharpen the question consider the energy-momentum tensor $\Theta_{\mu \nu}$. In QCD one can write

$$
\Theta_{\mu \nu}(x)=\Theta_{\mu \nu}^{q}(x)+\Theta_{\mu \nu}^{g}(x)
$$

giving $\Theta_{\mu \nu}$ in terms of a piece $\Theta_{\mu \nu}^{q}$ written in terms of quark fields and a piece $\Theta_{\mu \nu}^{g}$ written in terms of gluon fields. Because of operator mixing the separation given by (2.32) depends on $\mu$, the scale at which the quark and gluon pieces are renormalized. The first moment of $F_{2}$ gives information as to how much of the light cone momentum of the proton is contained in the quark components. To see this we use

$$
\frac{1}{2 p_{+}^{2}}\left\langle p\left|\Theta_{++}\right| p\right\rangle=1
$$

and we define

$$
\begin{aligned}
& \frac{1}{2 p_{+}^{2}}\left\langle p\left|\Theta_{++}^{q}\right| p\right\rangle=x_{q} \\
& \frac{1}{2 p_{+}^{2}}\left\langle p\left|\Theta_{++}^{g}\right| p\right\rangle=x_{g} .
\end{aligned}
$$

$x_{q}$ and $x_{g}$ are the fractions of the proton's momentum contained by quarks and gluons respectively. But, using (1.17), (2.13) and charge symmetry

$$
\frac{5}{9} x\left[q_{u}\left(x, Q^{2}\right)+\bar{q}_{u}\left(x, Q^{2}\right)+q_{d}\left(x, Q^{2}\right)+\bar{q}_{d}\left(x, Q^{2}\right)\right]=F_{2}^{P}\left(x, Q^{2}\right)+F_{2}^{N}\left(x, Q^{2}\right)
$$


with the $P$ and $N$ superscripts referring to proton and neutron, respectively. The operator product expansion relates the integral of the second moment of $q+\bar{q}$ to the matrix element of $\Theta_{++}^{q}$ so that one obtains

$$
x_{q}=\frac{9}{5} \int_{0}^{1} d x\left(F_{2}^{P}\left(x, Q^{2}\right)+F_{2}^{N}\left(x, Q^{2}\right)\right) \approx 0.45 .
$$

That is, quarks only carry about one-half the momentum of the proton at least when the renormalization scale $\mu^{2} \approx Q^{2}$ is above a few $(\mathrm{GeV})^{2}$.

We can reconcile the constituent quark model and the parton model if we understand that the constituent quark model must refer to static or nearly static properties of the nucleon. The constituent quark itself consists of bare quarks and gluons (partons) which are revealed in hard interaction like deeply inelastic scattering. Thus the momentum sum rule tells us that a constituent quark consists of a bare quark core, which may carry about half of the lightcone momentum, along with a quark-antiquark sea and gluons. Thus if we try to evaluate the local quark operator $\Theta_{++}^{q}$ in terms of constituent quarks we only get the correct answer to within factor of two.

What about vector current $j_{\mu}^{f}(x)=\tilde{q}_{f}(x) \gamma_{\mu} q_{f}(x)$ ? Can we get reliable results by evaluating forward or nearly forward matrix element of $j_{\mu}^{f}$ in terms of constituent quarks? That is does

$$
j_{\mu}^{f} \equiv \tilde{q}_{f} \gamma_{\mu} q_{f} \approx \tilde{Q}_{f} \gamma_{\mu} Q_{f}=J_{\mu}^{f}
$$

with $Q^{f}$ a constituent quark of flavor $f$ and with baryonic matrix elements of $J_{\mu}^{f}$ evaluated in terms of a simple three quark bound state model?

Here the experimental situation is more encouraging. Magnetic moments of baryons are well given in terms of the Dirac moments of the constituent quarks. Theoretically the situation is also more favorable. A constituent quark consists a bare core along with a bare quark-anti-quark sea and gluons. 
However, gluons carry no flavor quantum numbers and the sea cancels out in single current matrix elements of $j_{\mu}$. Thus in this case evaluation of matrix elements of $j_{\mu}^{f}$ in terms of $J_{\mu}^{f}$ should be, and is, much more reliable.

Finally we come to the case of axial vector current $j_{5 \mu}^{f}=\tilde{q}_{f} \gamma_{\mu} \gamma_{5} q_{f}$. Let us suppose the bare mass of the $u, d$ and $s$ quarks is zero, a not unreasonable approximation. Then $\partial_{\mu} j_{5 \mu}^{f}=0$. Nevertheless, even though the bare currents are conserved, there is no conserved axial charge. When a bare quark propagates in the vacuum it may interact with the condensate, $\bar{q} q$, representing spontaneous chiral symmetry breaking, and through this interaction the bare quark obtains a mass and undergoes changes in helicity. But, the axial charge is helicity so that the axial charge is not really conserved. Thus we would expect the necessity of a renormalization between $j_{5 \mu}$ and $J_{5 \mu}$ since the axial charge given by the two cannot be expected to be the same. Indeed we expect an independent renormalization for the flavor singlet and flavor octet parts of $j_{5 \mu}$

$$
j_{5 \mu}^{0}=Z_{\underline{0}} J_{5 \mu}^{0}, \quad j_{5 \mu}^{\frac{8}{5}}=Z_{\underline{8}} J_{5 \mu}^{\frac{8}{\mu}}
$$

with $\underline{0}$ and $\underline{8}$ referring to singlet and octet, respectively. $Z_{\underline{8}}$ can be determined from $G_{A} / G_{V}$ in the non-relativistic quark model and one gets $Z_{\underline{8}} \approx 3 / 4$. (If relativistic corrections in the quark model are taken into account $Z_{\underline{8}}$ may be somewhat larger than 3/4.) Thus the "naive" identification $j_{5 \mu}^{\frac{8}{5}}=J_{5 \mu}^{\frac{8}{\mu}}$ is not too bad for static baryonic matrix elements and a small renormalization, $Z_{\underline{8}} \approx 3 / 4$, bring all octet static matrix elements into excellent agreement with the non-relativistic quark model.

Thus we have seen that even though constituent quarks and partonic quarks are quite different objects the evaluation of baryonic matrix elements of bare (partonic) quark operators in terms of constituent quark operators gives reasonable results. The mismatch of a factor of two in relating bare and 
constituent quark energy-momentum tensors was the worst case we have met. So far, however, we have not discussed $j_{5 \mu}^{0}$, perhaps the most interesting case of all.

Much of our discussion in the early part of this chapter was aimed at showing that nucleonic matrix elements of $j_{5 \mu}^{0}$ are not directly related to bare quark quantities, but involve a gluonic component also. Thus

$$
\left\langle p s\left|j \frac{0}{5 \mu}\right| p s\right\rangle=2 M s_{\mu}\left(\Delta q-\frac{\alpha_{s} N_{f}}{2 \pi} \Delta g\right)
$$

corresponds to (2.31) where $\Delta q$ represents the fraction of the proton's helicity carried by quarks while $\Delta g$ is the amount of helicity carried by gluons. The $-\frac{\alpha_{s} N_{f}}{2 \pi} \Delta g$ term comes about from the flavor singlet axial vector anomaly as we have shown earlier.

Now the question is whether we should try to identify $j \frac{0}{5 \mu}$ with $J_{5 \mu}^{0}$ or whether we should only identify the quark partonic part of $j_{5 \mu}^{0}$ with $J_{5 \mu}^{0}$. Since $J_{5 \mu}^{0}$ is imagined to be the axial current due to a bare quark core, along with its gluons and its sea, it seems most natural to try and identify $J_{5 \mu}^{0}$ with the $\Delta q$ part of the right hand side of (2.36). After all it is $\Delta q$ which could be the bare quark core around which the constituent quark may be built while the $-\frac{\alpha_{s} N_{f}}{2 \pi} \Delta g$ has no bare quark part at all. After briefly discussing the experimental situation with respect to the singlet axial current matrix elements we shall conclude our discussing of spin and the axial anomaly.

\subsection{The Experimental Situation}

The new ingredient which so much stimulated the field of spin dependence in high energy reactions was the EMC experiment 12] on spin dependent DIS. 
The result of that experiment is that

$$
\int_{0}^{1} g_{1}\left(x, Q^{2}\right)=0.126 \pm 0.010 \pm 0.015
$$

with $g_{1}$ the spin dependent structure function of the proton. For the EMC experiment $\left\langle Q^{2}\right\rangle \approx 11 \mathrm{GeV}^{2}$. Using (2.37) with (2.31) one obtains

$$
\frac{1}{18}[4 \Delta u+\Delta d+\Delta s]-\frac{\alpha_{s} N_{f}}{12 \pi} \Delta g=0.126 \pm 0.010 \pm 0.015 .
$$

There are two other pieces of information in hand. Semileptonic hyperon decay is well fit using flavor $S U(3)$ symmetry 13 . In the analysis of such decays one determines, after doing an $S U(3)$ flavor rotation, the proton matrix element of the eighth component of the octet axial vector current. Expressed in parton language

$$
3 F-D=\Delta u+\Delta d-2 \Delta s .
$$

In addition neutron $\beta$-decay along with an isospin rotation determines the proton matrix element of the third component of the axial vector current as

$$
\Delta u-\Delta d=G_{A}=F+D .
$$

Using $F=0.47 \pm 0.04$ and $D=0.81 \pm 0.03$ one obtains from (2.37) (2.40)

$$
\begin{aligned}
& \Delta u-\frac{\alpha_{s} \Delta g}{2 \pi}=0.78 \pm 0.08 \\
& \Delta d-\frac{\alpha_{s} \Delta g}{2 \pi}=-0.50 \pm 0.08 \\
& \Delta s-\frac{\alpha_{s} \Delta g}{2 \pi}=-0.16 \pm 0.08
\end{aligned}
$$


Adding the above one gets

$$
\Delta u+\Delta d+\Delta s-\frac{3 \alpha_{s}}{2 \pi} \Delta g=0.13 \pm 0.19 .
$$

If we were to neglect the $\frac{\alpha_{s} \Delta g}{2 \pi}$ terms in (2.41) and (2.42) then (2.41C) would tell us that strange quarks carry about $16 \%$ of the proton's spin but with an opposite sign while (2.42) would say that all quarks and anti-quarks only carry about $13 \%$ of the spin. Each of these would be rather striking conclusions. We might have guessed, based on our discussion of the last section, that quarks should carry at least $50 \%$ of the proton's spin, and we probably would have guessed that strange quarks should carry less than about $5 \%$ of the proton's spin. Of course the errors in (2.41) and (2.42) are large, so that it is very important to bear in mind that even with a neglect of $\Delta g$ there may not be a real conflict with the intuition we have gained from the constituent quark model.

If the central values in (2.41) and (2.42) are in fact the correct values, then either $\Delta g$ is moderately large, or the constituent quark model simply fails. $\Delta g \approx 3$ would reduce the central value of $\Delta s$ from -0.16 to a value close to -0.06 and it would increase the central value of $\Delta u+\Delta d+\Delta s$ from 0.13 to about 0.45 . Each of these numbers is marginally acceptable in the constituent quark model. If it should turn out that $\Delta g$ is very small and that the central values given in (2.41) and (2.42) are accurate, then the constituent quark model will have suffered a real defeat and it may be reasonable to search for the smallness of $\Delta u+\Delta d+\Delta s$ in terms of a Skyrme-type model 14 .

Finally, it is important to emphasize that $\Delta g$ is a measurable quantity. In spin dependent deep inelastic scattering the $\Delta g$ term given in (2.18) should be the main source of two jet events after the first moment, in terms of the Bjorken- $x$-variable, is taken. 


\section{Bibliography}

1. J.S. Bell and R. Jackiw. Nuovo Cimento, a60:47, 1969.

2. S.L. Adler. Phys. Rev., 177:2426, 1969.

3. A.V. Efremov and O.V. Teryaev. Dubna preprint E2-88-287.

4. G. Altarelli and G.G. Ross. Phys.Lett., 212B:391, 1988.

5. R.D. Carlitz, J.C. Collins and A.H. Mueller. Phys. Lett., 214B:229, 1988. In Proceedings of the $24^{\text {th }}$ Recontre de Moriond, ed. J.Tran Thanh Van, Editions Frontieres, Gif-sur-Yvette, 1989.

6. G. Altarelli and W.J. Stirling., Particle World, 1:40, 1989.

7. G.G. Ross and R.G. Roberts. Rutherford preprint RAL-90-062, 1990.

8. J. Kodaira. Nucl. Phys., B165:129, 1980.

9. G.'t Hooft and M. Veltman. Nucl. Phys., B44:189, 1972.

10. J.C. Collins. "Renormalization", Cambridge University Press, Cambridge, 1984, section 13.2 .

11. See, for example, S.J. Brodsky and G.P. Lepage. In Perturbative Quantum Chromodynamics, ed. A.H. Mueller, World Scientific, Singapore, 1989 .

12. EMC, J. Ashman et al.. Nucl. Phys., B328:1, 1989.

13. M.Bourquin et al.. Z. Phys., C21:27, 1983.

14. S.J. Brodsky, J. Ellis and M. Karliner. Phys. Lett., 206B:309, 1988. 


\section{Chapter 3}

\section{Exclusive Reactions and Color Transparency in Almost Exclusive Reactions on Nuclei}

\section{Contents}

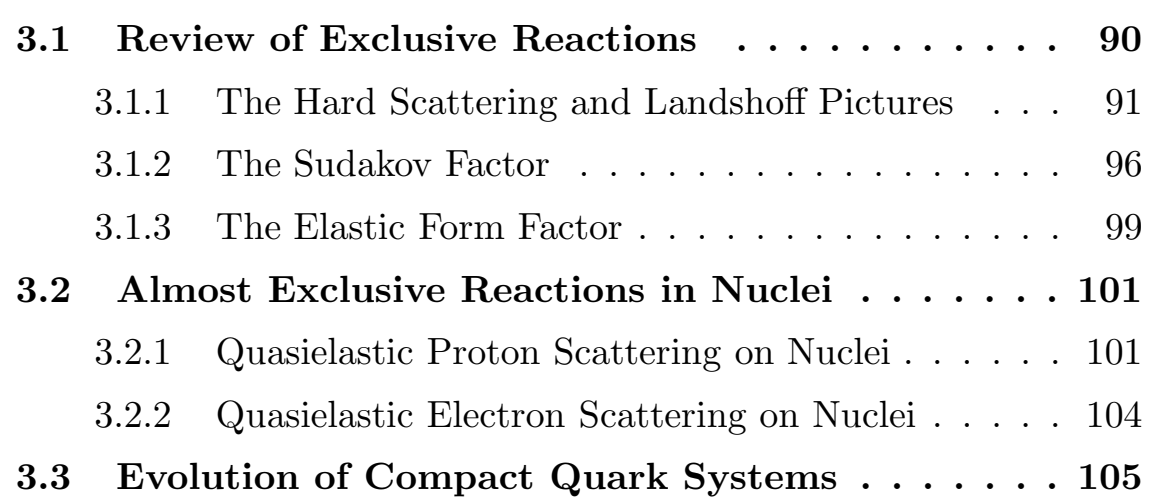


$3.3 .1 \quad$ Classical Expansion . . . . . . . . . . . . 106

3.3 .2 Quantum Expansion . . . . . . . . . . . . . . 108

Bibliography . . . . . . . . . . . . . 111

Exclusive hadronic reactions have been intensively studied in the context of QCD since the late 1970's 1. Although the formalism for dealing with such reactions as elastic electron-nucleon scattering and high energy elastic hadronhadron scattering is still under active development and improvement the basic ingredients are now well understood. In particular the space time picture of high energy exclusive reactions is understood and it is really the testing of that picture which is our concern in this Chapter. Indeed quantitative predictions for exclusive reactions will never be comparable in accuracy to those for inclusive hard reactions. It may well be that the best one can ever hope for is a qualitative and perhaps a semi-quantitative testing of the QCD predictions for exclusive reactions.

\subsection{Review of Exclusive Reactions}

In this section the basic picture underlying exclusive reactions will be discussed in a qualitative way. We begin by considering high energy wide angle elastic pion-pion scattering after which we shall derive the counting rules for a general high energy elastic scattering. 


\subsubsection{The Hard Scattering and Landshoff Pictures}

There are two competing physical pictures of $\pi-\pi$ wide angle elastic scattering 3 . Let us refer to them as the hard scattering picture and the Landshoff picture respectively. The process is illustrated in Fig. 3.1 where the incoming momenta are $p_{1}$ and $p_{2}$ and the outgoing momenta are $p_{1}{ }^{\prime}$ and $p_{2}{ }^{\prime}$. In the center of mass we may write

$$
\begin{aligned}
p_{1}=(E, 0,0, p)=\left(E, \vec{e}_{z} p\right), & p_{2}=(E, 0,0,-p)=\left(E,-\vec{e}_{z} p\right) \\
p_{1}{ }^{\prime}=(E, \overrightarrow{e p}), & p_{2}{ }^{\prime}=(E,-\overrightarrow{e p})
\end{aligned}
$$

where $\vec{e}_{z}=\cos \Theta$ gives the angle of scattering and $s=\left(p_{1}+p_{2}\right)^{2}$.

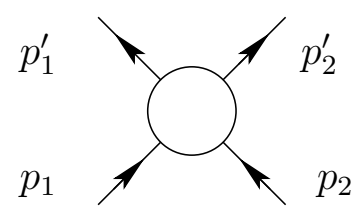

Figure 3.1: The kinematics of wide angle elastic scattering.

Suppose $t \approx 0$ is the time at which the scattering takes place. We shall soon see that in both the hard scattering and in the Landshoff pictures an elastic event can only occur if the incoming pions consist solely of a valence quark and antiquark at the time of the scattering. That is, only a particular part of the wave function of the pion is capable of carrying out an elastic scattering. Then in the hard scattering picture the scattering proceeds as illustrated in Fig. 3.2. Before the collision takes place the valence quark and antiquark of each pion come close together so that during the time of collision, $\Delta t \sim 1 / \sqrt{s}$, the two quarks and the two antiquarks from the initial pions are within region $|\Delta \vec{x}| \lesssim 1 / \sqrt{s}$. The collision then serves to rearrange the 
momenta of the quarks and antiquarks so that the outgoing quark-antiquark systems have the momenta $p_{1}{ }^{\prime}$ and $p_{2}{ }^{\prime}$ respectively.

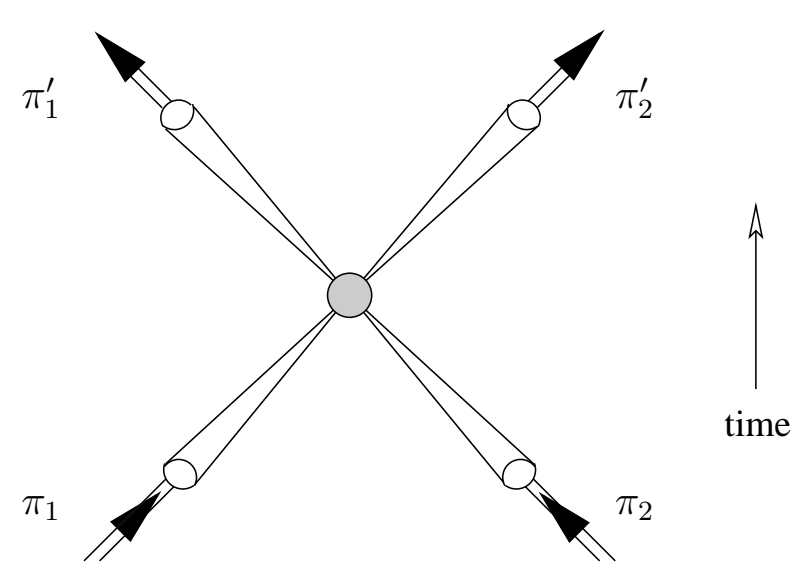

Figure 3.2: Collapse and later expansion of the pion wavefunctions in wide angle elastic scattering.

Thus in the hard scattering picture we may write

$$
d \sigma=P_{r}(1) P_{r}(2) P_{r}\left(1^{\prime}\right) P_{r}\left(2^{\prime}\right) \sigma_{H S} d \Omega
$$

where $P_{r}(1)$ is the probability that the quark and antiquark in the pion having $p_{1}$ are within a spatial distance $\left|\Delta \overrightarrow{x_{1}}\right|=1 / \sqrt{s}$. Now having $\left|\Delta z_{1}\right| \lesssim 1 / \sqrt{s}$ is automatic because the pion wavefunction is Lorentz contracted due to the high momentum of the pion. Neglecting the QCD logarithms due to evolution, the probability that the transverse components of the quark and antiquark be found in a region $\left|\Delta x_{\perp}\right| \lesssim 1 / \sqrt{s}$ is just geometric, $P_{r}(1) \propto$ $\left(\frac{1}{\sqrt{s}}\right)^{2} / 1 \mathrm{fm}^{2} \propto 1 / s$. Similarly $P_{r}\left(1^{\prime}\right), P_{r}(2)$ and $P_{r}\left(2^{\prime}\right)$, the probabilities to 
find the valence quark and antiquark of the pions $\pi_{1}{ }^{\prime}, \pi_{2}$ and $\pi_{2}{ }^{\prime}$ each within a size $|\Delta \vec{x}| \lesssim 1 / \sqrt{s}$ are of magnitude $1 / s$ also. $\sigma_{H S}$ is the hard scattering cross section. Since $\sigma_{H S}$ refers to the scattering of compact quark antiquark pairs it must scale according to the energy of the reaction since there is no other scale available. Thus $\sigma_{H S} \sim 1 / s$. Using $d \Omega=(4 \pi) / s d t$, with $t=\left(p_{1}-p_{1}{ }^{\prime}\right)^{2}$ one finds

$$
\frac{d \sigma}{d t} \propto \frac{1}{s^{6}},
$$

the dimensional counting rule derived some time ago 213 .

If, instead of considering $\pi_{1}+\pi_{2} \rightarrow \pi_{1}{ }^{\prime}+\pi_{2}{ }^{\prime}$ one considers a general elastic reaction $h_{1}+h_{2} \rightarrow h_{3}+h_{4}$ where the minimum number of constituents of $h_{i}$ is $n_{i}$ then clearly

$$
\frac{d \sigma}{d t} \propto P_{r}\left(h_{1}\right) P_{r}\left(h_{2}\right) P_{r}\left(h_{3}\right) P_{r}\left(h_{4}\right) \frac{1}{s^{2}}
$$

in the hard scattering picture. But $P_{r}\left(h_{i}\right)$ is just determined geometrically by the probability to find $n_{i}$ constituents in a transverse region $\left|\Delta x_{\perp}\right| \sim 1 / \sqrt{s}$. Thus $P_{r}\left(h_{i}\right) \propto s^{-n_{i}+1}$ so that

$$
\frac{d \sigma}{d t} \propto s^{-\left(n_{1}+n_{2}+n_{3}+n_{4}\right)+2}
$$

in the hard scattering picture. For elastic nucleon-nucleon scattering the dimensional counting rule becomes $d \sigma / d t \propto s^{10}$.

In the hard scattering picture the essential part of that picture is that over a time $\Delta t \sim 1 / \sqrt{s}$ all the constituents making up the initial, or final, state be found in the some small, $|\Delta \vec{x}| \sim 1 / \sqrt{s}$, region of space. On the other hand in the Landshoff picture different constituents carry out hard scatterings in different regions of space, but again over a short interval, $\Delta t \sim 1 / \sqrt{s}$, of time. 
To begin consider the case of $\pi-\pi$ elastic scattering. Suppose the scattering takes place in the $\mathrm{x}-\mathrm{z}$ plane. Then in order that an elastic reaction take place it is necessary that all the quarks and antiquarks of the initial pions be found in the region $|\Delta x| \lesssim 1 / \sqrt{s},|\Delta z| \lesssim 1 / \sqrt{s}$, but it is not kinematically necessary that $\Delta y$ be smaller than the normal extent of the pion wavefunction. Thus in the Landshoff picture we suppose that the quarks and anti-qarks carry out hard collisions near $\mathrm{t}=0$, with $\left|x_{i}-x_{j}\right|$ and $\left|z_{i}-z_{j}\right|$ being of size $1 / \sqrt{s}$ during the collision for all quarks and antiquarks $i$ and $j$. However, we suppose that in the y-direction there are two separate collision points where, at each collision point, one quark (or antiquark) from each initial pion carries out a hard elastic reaction. In this case we may write

$$
d \sigma \propto \bar{P}_{r}(1) \bar{P}_{r}(2) \bar{P}_{r}\left(1^{\prime}\right) \bar{P}_{r}\left(2^{\prime}\right) \bar{\sigma}_{H S}^{(x)} \bar{\sigma}_{H S}^{(y)} d \Omega^{(1)} d \Theta_{y}^{(2)}
$$

where now $\bar{P}_{r} \sim 1 / \sqrt{s}$ since one need only require that the $\mathrm{x}$ coordinates of the valence quark and antiquark be within a size $1 / \sqrt{s} . \bar{\sigma}_{H S}^{(x)}$ is the scattering "cross section" for the $\mathrm{x}$ coordinates of the two quarks in one incoming pion to overlap the two quarks in the other incoming pion. This is given geometrically as $\bar{\sigma}_{H S}^{(x)} \sim 1 / \sqrt{s}$ since the quark-antiquark pair in a given pion are within a distance $\Delta x \sim 1 / \sqrt{s} . \bar{\sigma}_{H S}^{(y)}$ is the overlap probability in the y-direction. Here the quark-antiquark pairs for the two initial pions are not compact so there is a geometrical factor for each of the two quark-quark collisions, each collision involving a quark (antiquark) from one of the incoming pions with a quark (antiquark) from the other incoming pion. Thus $\bar{\sigma}_{H S}^{(y)} \propto 1 / s . d \Omega^{(1)}$ is the element of solid angle for one of the quark (or antiquark) collisions. $d \Theta_{y}^{(2)}$ is the small angular region allowed, in a direction out of the $\mathrm{x}-\mathrm{z}$ plane, for the second hard quark (or antiquark) collision $d \Theta_{y}^{(2)} \lesssim 1 / \sqrt{s}$ in order that the hard scatterings give outgoing quarks having $\Delta y \sim 1 \mathrm{fm}$ and $\Delta p_{y} \lesssim 1 / \mathrm{fm}$. 
Using $d \Omega^{(1)} \propto d t / s$ one finds from (3.3.)

$$
\frac{d \sigma}{d t} \sim \frac{1}{s^{5}}
$$

In general for $h_{1}+h_{2} \rightarrow h_{1}^{\prime}+h_{2}^{\prime}$ with $h_{1}$ and $h_{1}^{\prime}$ having $n_{1}$ valence quarks and antiquarks and $h_{2}$ and $h_{2}^{\prime}$ having $n_{2}$ valence quarks and antiquarks a formula similar to (3.3) can be written. Suppose $n_{1} \leq n_{2}$ then there can be $n_{1}$ distinct quark (or anti- quark) scatterings located at $n_{1}$ points along the y-axis. Then

$$
\begin{array}{cl}
\qquad \frac{d \sigma}{d t}=\bar{P}_{r}(1) \bar{P}_{r}(2) \bar{P}_{r}\left(1^{\prime}\right) \bar{P}_{r}\left(2^{\prime}\right) \bar{\sigma}_{H S}^{(x)} \bar{\sigma}_{H S}^{(y)}\left[d \Theta_{y}\right]^{n_{1}-1} \\
\text { where now } & \bar{P}_{r}\left(1^{\prime}\right), \bar{P}_{r}(1) \propto(1 / \sqrt{s})^{n_{1}-1} \\
\text { and } & \bar{P}_{r}(2), \bar{P}_{r}\left(2^{\prime}\right) \propto(1 / \sqrt{s})^{n_{1}-1}(1 / \sqrt{s})^{n_{2}-n_{1}} .
\end{array}
$$

The factor $(1 / \sqrt{s})^{n_{2}-n_{1}}$ in $\bar{P}_{r}(2)$ and $\bar{P}_{r}\left(2^{\prime}\right)$ reflects the fact that there can only be $n_{1}$ distinct scattering points on the y-axis so that $n_{2}-n_{1}$ quarks (or antiquarks) in $h_{2}$ and $h_{2}^{\prime}$ must be close, $\Delta y \lesssim 1 / \sqrt{s}$, to some other quark (or antiquark) in $h_{2}$ and $h_{2}^{\prime} . \bar{\sigma}_{H S}^{(x)} \sim 1 / \sqrt{s}$ as before while now $\bar{\sigma}_{H S}^{(y)} \sim(1 / \sqrt{s})^{n_{1}}$. Thus

$$
\frac{d \sigma}{d t} \propto\left(\frac{1}{s}\right)^{2 n_{1}+n_{2}-1}
$$

for $h_{1}+h_{2} \rightarrow h_{1}^{\prime}+h_{2}^{\prime}$ with $n_{1} \leq n_{2}$ and $n_{1}$ and $n_{2}$ being the minimum number of constituents in hadrons 1 and 2 respectively. Thus for elastic nucleonnucleon scattering the Landshoff process leads to $d \sigma / d t \propto(1 / s)^{8}$ while for pion-nucleon scattering it leads to $(1 / s)^{6}$.

From the discussion above and from the resulting behaviour for wide angle elastic scattering in the hard scattering picture and in the Landshoff picture, 
equations (3.2), (3.5) respectively, it should be clear that wide angle alastic scattering proceeds through the Fock space component of the hadrons wavefunction containing the minimum number of quanta. Higher numbers of quanta partaking in the scattering will simple raise the various $n_{i}$ appearing in (3.2) and (3.5).

\subsubsection{The Sudakov Factor}

From our disscusion in the previous section it would appear that high energy wide angle elastic scattering is dominated by a set of essentially independent hard partonic scatterings, the independent scatterings separated spatially by about one fermi. This is the Landshoff picture. Before discussing the role of the Sudakov form factor in the present circumstance let's go back for a moment to $\pi-\pi$ scattering and relate the Feynman diagram calculation to the hard scattering and Landshoff pictures we have just described.

In Fig. 3.3 a particular Feynman diagram contribution to $\pi-\pi$ scattering is shown.

In the hard scattering picture the gluon lines $k_{1}-k_{1}^{\prime}, p_{1}-p_{1}^{\prime}-k_{1}+k_{1}^{\prime}$ and $k_{2}-k_{2}^{\prime}$ as well as the quark lines $k_{1}+k_{2}-k_{1}^{\prime}, p_{2}^{\prime}-k_{2}$ have virtuality on the order of $s$. That is $\left(k_{1}-k_{1}^{\prime}\right)^{2} \propto s$, etc. This large virtuality is what requires the coordinate positions of the quarks involved in the scattering to be close together. This is a general result. If a QCD hard scattering analysis is to be applicable there must be a connected set of Feynman lines, each of virtuality $s$, to which the external particles attach through exclusive QCD wavefunctions. In the $\pi-\pi$ scattering case that connected set of Feynman lines is the set $k_{1}-k_{1}^{\prime}$, etc. enumerated above.

Now in wide angle, $\pi-\pi$ elastic scattering the kinematics of the process does not require that all of the above enumerated lines be of large virtuality. The two fermion lines $k_{1}+k_{2}-k_{1}^{\prime}$ and $p_{2}^{\prime}-k_{2}$ along with the gluon line $k_{2}-k_{2}^{\prime}$ 


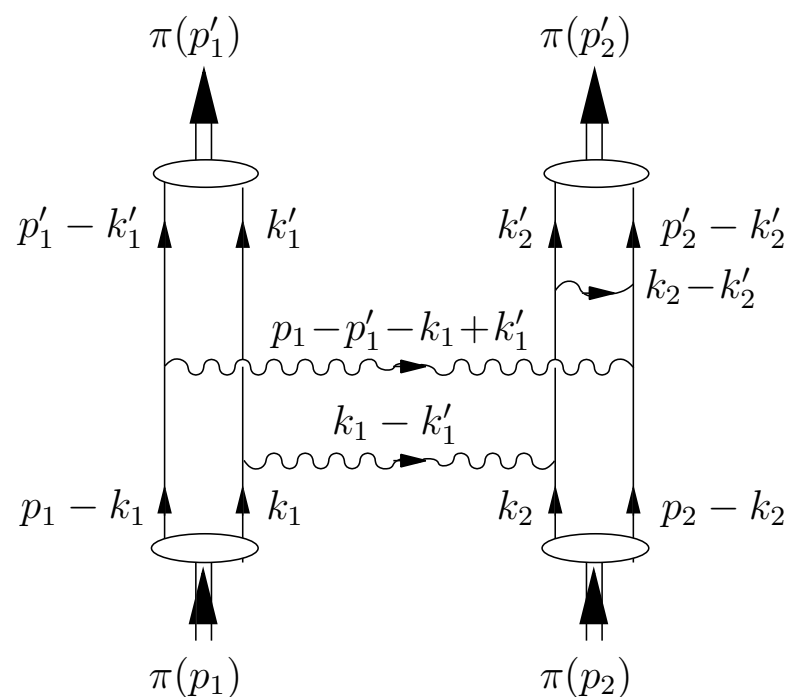

Figure 3.3: The kinematics of wide angle elastic scattering.

need not have large virtuality. In this case the resulting hard scattering lines $k_{1}-k_{1}^{\prime}$ and $p_{1}-p_{1}^{\prime}-k_{1}+k_{1}^{\prime}$ are disconnected and so the hard scattering does not occur within a small region of space-time. Indeed, in the graph shown in Fig. 3.3 the virtuality of the lines $k_{1}+k_{2}-k_{1}^{\prime}, p_{1}^{\prime}-k_{2}$ and $k_{2}-k_{2}^{\prime}$ can vary from $1 / r_{0}^{2}$ to $s$, where $r_{0}$ is the radius of the pion. Thus the hard scattering and Landshoff regions are smoothly connected.

We have said that additional quanta in the wavefunction of the incoming pions at the time of scattering will lead to power behaviors more strongly suppressed than those given by (3.2) and (3.5). In the hard scattering picture it is natural not to have additional gluons present at the time of scattering since the pion wavefunctions are very compact and thus color neutral down 
to distances of size $|\Delta \vec{x}| \sim 1 / \sqrt{s}$. However, in the Landshoff picture the quark and antiquark in each of the incoming pions are separated by an amount $|\Delta y| \sim 1 \mathrm{fm}$ so that the pion is globally color neutral but not locally color neutral. Thus it is natural that the individual quarks and antiquarks in the incoming pions have their usual cloud of virtual gluons at the time of the hard scattering.

In order that the hard scattering take place with the power behavior given by (3.5) it is necessary to pick out that part of the pion wavefunction consisting only of a valence quark-antiquark pair. However, the probability of finding a part of the wavefunction of a high energy pion consisting only of a quark and an antiquark with no gluons is very small so that the power law (3.5) cannot be maintained at very high energy. This is the Sudakov suppression (see Chapter 11). It can also be understood classically since independent, and spatially separated, wide angle scatterings of charged particles are always accompanyied by a significant amount of radiation. But this radiation would mean that the process was not truly elastic. In the hard scattering picture, on the other hand, there is no large change in color currents at the hard scattering since the incoming, and outgoing, pions are color neutral down to distances of size $|\Delta \vec{x}| \sim 1 / \sqrt{s}$.

Thus at very high energy the Sudakov suppression brings one very close to the hard scattering picture. A careful treatment of the hard scattering and Landshoff regions leads to 56

$$
\frac{d \sigma}{d t} \propto s^{-5-2 c \ln \left(1+\frac{1}{2 c}\right)}
$$

for $\pi-\pi$ scattering and

$$
\frac{d \sigma}{d t} \propto s^{-8-3 c \ln \left(1+\frac{2}{3 c}\right)}
$$

for nucleon-nucleon scattering with $c=8 C_{F} /\left(11-\frac{2}{3} N_{f}\right)$, results very close 
to the hard scattering picture.

\subsubsection{The Elastic Form Factor 1}

The elastic electromagnetic form factor is somewhat simpler to study in QCD than elastic hadron-hadron scattering because there is no region analogous to the Landshoff region. In order to describe the physical picture underlying our understanding of the form factor it is convenient to use a frame where, for the pion form factor, the incoming and outgoing momenta take the form

$$
p=\left(p+\frac{m^{2}}{2 p}, 0,0, p\right), \quad p^{\prime}=\left(p+\frac{m^{2}}{2 p}, 0,0,-p\right)
$$

with the process indicated in Fig. 3.4, where the virtual photon is the line having momentum $q$. In that figure we have shown how the process proceeds in QCD.

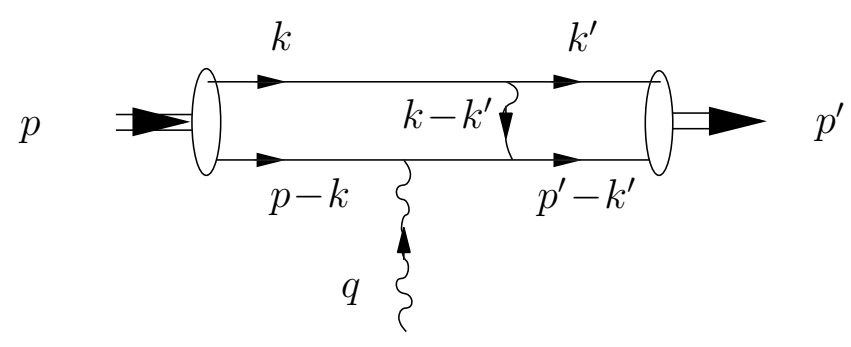

Figure 3.4: The elastic form factor of the pion.

Only the minimum valence quark-antiquark portion of the pion wavefunction is important in evaluating the form factor. The argument is the following: In the frame indicated in (3.8) the separation in the $z$-direction of the quark 
and antiquark in the incoming pion is $\Delta z \sim 1 / p=2 / Q$. The virtual photon is absorbed over a period of time, $\Delta t$, given by $\Delta t \sim 1 / Q$. Suppose the photon is absorbed by the quark. Then the direction of the quark is immediately changed from moving in the $+z$ to moving in the $-z$ direction. But, if the quark and antiquark separate by an amount $|\Delta z| \gg 1 / Q$ they will not be able to get together to form the outgoing pion. In order that the quark and antiquark be able to communicate before $\delta z$ becomes grater than $1 / Q$ they must be within the transverse size $\Delta x_{\perp} \lesssim 1 / Q$ as information cannot travel faster than the velocity of light. Thus at the time of the absorption of the photon the lines $p-k$ and $k$ must be within a distance $|\Delta \vec{x}| \lesssim 1 / Q$, exactly the hard scattering configuration we discussed earlier in our consideration of elastic scattering.

Technically the exchange of the gluon, $k-k^{\prime}$, between the quark and antiquark is what turns the antiquark around. The fact that $\left(k-k^{\prime}\right)^{2} \sim Q^{2}$ is the region of momentum which dominates the Feynman diagram calculation of the graph in Fig. 3.4 also indicates that the quark and antiquark must be close together at the time of absorption of the virtual photon.

For the elastic form factor it is possible to give rather compact form for the asymptotic behavior 1. For the pion form factor the answer is

$$
F_{\pi}\left(Q^{2}\right) \rightarrow 16 \pi f_{\pi}^{2} \frac{\alpha_{s}\left(Q^{2}\right)}{Q^{2}}\left[1+\sum_{N=2,4,6}^{\infty} C_{N}\left(\ln \frac{Q^{2}}{\mu^{2}}\right)^{-\gamma_{N}}\right]+O\left(\frac{1}{Q^{4}}\right)
$$

where the $\gamma_{N}$ are known positive numbers. However, the $C_{N}$ depend on the wavefunction of the pion in a non-perturbative way and can only be determined either through a model calculation or through fits to the data.

In the nucleon case much work has gone into trying to restrict the $C_{N}$ by using QCD sum rules [ $_{a L 2.08]}$. How successful this will ultimately be is not 
yet clear. It may be that large order $1 / Q^{4}$ terms in $F_{\pi}$ and $1 / Q^{6}$ terms in the nucleon form factor will ultimately make quantitative comparison between theory and experiment extremely difficult 9 .

\subsection{Almost Exclusive Reactions in Nuclei}

One way to test the QCD picture of hard scattering described in the previous section would be to make quantitative comparisons between theory and experiment. This is very difficult to do with our present lack of knowledge of both the $Q^{2}$ 's necessary to apply the asymptotic formulas 9 and of the non-perturbative aspects of the hadronic wavefunctions which are necessary to do precise calculations. It does, however, seem possible to test the QCD picture of exclusive reactions by considering quasielastic reactions on nuclei.

\subsubsection{Quasielastic Proton Scattering on Nuclei}

Consider the reaction 10 12

$$
p_{1}+A \rightarrow p_{1}^{\prime}+p_{2}^{\prime}+(A-1)^{*}
$$

with $p_{1}, p_{1}^{\prime}$ and $p_{2}^{\prime}$ protons and $(A-1)^{*}$ a nucleus, possibly in an excited state. The reaction indicated in (3.10) can occur only if the initial proton, $p_{1}$, can penetrate into the nucleus, interact with a proton, $p_{2}$, of the nucleus and carry out a hard elastic reaction. $p_{1}^{\prime}$ and $p_{2}^{\prime}$ must then leave the nucleus without having any inelastic reaction with other nucleons in the nucleus. At first sight the reaction seems very unlikely as one might expect the protons $p_{1}, p_{1}^{\prime}$ and $p_{2}^{\prime}$ to have many inelastic reactions while passing through the nucleus. However, on closer reflection one begins to see that (3.10) may not be so difficult to achieve. After all the QCD picture of wide angle elastic scattering demands 
that the incoming proton, $p_{1}$, be composed of three quarks having spatial separation $\Delta \vec{x}$ very small just before the collision. But if $\Delta \vec{x}$ is very small then the proton is nearly color neutral, in a local manner, just before the elastic scattering. But a nearly locally color neutral system interacts very weakly with nucleons and thus should be able to pass through the nucleus. Similar considerations apply to the outgoing protons $p_{1}^{\prime}$ and $p_{2}^{\prime}$. Thus the QCD picture of wide angle elastic scattering suggests that the quasielastic reaction (3.10) may be surprisingly large. Let's try to make this a little more precise. Define a transparency, $T$, according to

$$
T=\frac{\frac{d \sigma}{d \Omega}\left(p_{1}+A \rightarrow p_{1}^{\prime}+p_{2}^{\prime}+(A-1)^{*}\right)}{Z \frac{d \sigma}{d \Omega}\left(p_{1}+p_{2} \rightarrow p_{1}^{\prime}+p_{2}^{\prime}\right)}
$$

where the reaction indicated in the denominator of (3.11) is elastic protonproton scattering. Both reactions in (3.11) are assumed to be at high energy and at wide angle. If the incoming proton, $p_{1}$, or the outgoing protons, $p_{1}^{\prime}$ and $p_{2}^{\prime}$, are strongly absorbed while passing through the nucleus then $T$ will be much less than 1 . QCD predicts $T \approx 1$ at high energy, at least after fermi motion corrections are made to the numerator on the right hand side of (3.11).

We can get an estimate of the energies necessary to obtain a reasonable $T$ from the following qualitative argument: If the three quark system making up the proton $p_{1}$ has a transverse radius $r_{\perp}$ then one may expect that this system has an inelastic cross section with a normal nucleon equal to $\pi r_{\perp}^{2}$. Thus for $p_{1}, p_{1}^{\prime}$ and $p_{2}^{\prime}$ to interact weakly with the nucleons in the nucleus we need the momentum transfer of the reaction $\sqrt{-\left(p_{1}-p_{1}^{\prime}\right)^{2}}=\sqrt{-t}$ to be much greater than $\left(1 \mathrm{fm}^{-1}\right)$. In order that $r_{\perp}$ stay small while the incoming and outgoing protons pass through the nucleus one needs $p_{1} / M, p_{1}^{\prime} / M, p_{2}^{\prime} / M$ 
all to be much greater than one. (The rate of expansion of $r_{\perp}$ as the protons leave the center of the collision is slowed down by Lorentz time dilatation so that we must require $p / M$ be large enough so that $r_{\perp}$ be small over the complete traversal of the nucleus. The expansion of the three quark system as it leaves the collision point will be dealt with in more detail in the next section.) Thus for $\sqrt{-t}$ a few GeVand for $p_{1}, p_{1}^{\prime}$ and $p_{2}^{\prime}$ greater than a few $\mathrm{GeV}$ we might expect to see some signs of color transparency, though in order to get $T$ near 1 it may be necessary have $\sqrt{-t}$ values which are difficult to achieve in present experiments.

There has been one experiment 13 performed which shows an increase of $T$ between $p_{1}=3 \mathrm{GeV}$ and $p_{1}=9 \mathrm{GeV}$ and then a decrease of $T$ between $p_{1}=9 \mathrm{GeV}$ and $p_{1}=12 \mathrm{GeV}$. This variation of $T$ seems mostly due to the variation of the denominator on the right hand side of (3.11) rather than due to the numerator. This is clearly an indication that the asymptotic behavior for wide angle elastic proton-proton scattering has not been achieved yet at Brookhaven energies. The fact that elastic proton-proton scattering shows oscillations with energy at fixed angle while no such oscillations are apparent in the $p$-Nucleus data have led Ralston and Pire 14 to suggest that at present energies both the hard scattering and Landshhoff parts of the proton wavefunction are important. The oscillations in the proton-proton data would be due to an energy dependent phase relating the hard scattering and Landshoff amplitudes. In proton-nucleus quasielastic scattering the Landshoff part of the wavefunction would be filtered out by inelastic nuclear collisions so that only the hard scattering part of the wavefunction is effective. Thus no oscillation appear in proton-nucleus quasielastic scattering. This is a very interesting idea and is likely correct. However, it is important to understand the source of the energy dependent phase before the Ralston-Pire explanation can be considered convincing. 


\subsubsection{Quasielastic Electron Scattering on Nuclei}

Now consider the reaction

$$
e+A \rightarrow e^{\prime}+p^{\prime}+(A-1)^{*}
$$

where $p^{\prime}$ is a proton and $(A-1)^{*}$ is a (possibly) excited nuclear state, the possible excitations being summed over. Again one defines a transparency

$$
T_{\gamma}=\frac{F\left(\gamma(g)+A \rightarrow p^{\prime}+(A-1)^{*}\right)}{Z F\left(\gamma(g)+p \rightarrow p^{\prime}\right)},
$$

where as in (3.11) we neglect fermi motion effects for simplicity of discussion. The idea here is exactly the same as in the previous section. Naively one might expect $T_{\gamma}$ to be very small. However, if the hard scattering picture is correct $T_{\gamma}$ should approach one at very large $Q^{2}$. Even at not so large $Q^{2}$ one might hope to see $T$ increase with $Q^{2}$.

Let us briefly review the kinematics of the reaction $\gamma(g)+p \rightarrow p^{\prime}$ where in the laboratory system

$$
\begin{aligned}
p & =(M, 0,0,0) \\
q & =\left(\frac{Q^{2}}{2 M}, 0,0, Q \sqrt{\frac{Q^{2}}{4 M^{2}}+1}\right) \\
p^{\prime} & =\left(\frac{Q^{2}}{2 M}+M, 0,0, Q \sqrt{\frac{Q^{2}}{4 M^{2}}+1}\right) .
\end{aligned}
$$

Thus the outgoing proton has a momentum equal to $Q^{2} / 2 M$ when $Q^{2} / M^{2} \gg$ 1. Thus for $Q \gtrsim 2-3 \mathrm{GeV}$ the outgoing proton is reasonably relativistic. Ideally one would like $Q$ very large in order to test the hard scattering picture 
of QCD by finding $T=1$, at least after corrections due to fermi motion are taken into account. However, even at lower $Q$ 's one should be able to see the hard scattering picture emerge by observing an increase in $T$ as $Q^{2}$ grows. The advantage of quasielastic electron scattering compared to quasielastic proton-nucleus scattering is that the denominator in (3.12) is known to be smoothly varying while the denominator in (3.11) oscillates with energy.

If experiments do find an increase of $T$ with $Q^{2}$ then it may be possible to measure (3 quark)-nucleus cross sections, with the three quarks in a compact state, by studying the variation of $T$ with atomic number $A$. This could give information on the way the newly born three quark system evolves into a proton, which topic is the subject of the next section of this Chapter.

\subsection{Evolution of Compact Quark Systems into Hadrons}

For expliciteness let us consider quasielastic electron-nucleus scattering. Our physical picture of how the reaction should occur at high $Q^{2}$ is as follows. The virtual photon $(q)$ is absorbed by a quark in a proton $(p)$ at rest except for fermi motion which we ignore in this discussion. In the laboratory system the proton $(p)$ need not be in a compact three valence configuration as the hard part of the process takes place over time scales on the order of one fermi. After a time $\Delta t \sim 1 \mathrm{fm}$ the three quark system is compact $\Delta x_{\perp} \sim 1 / Q, \Delta z \sim 1 / Q^{2}$ and has momentum $p^{\prime}$ as given by (3.13c). The three quark system then expands in the transverse direction as it moves through the nucleus and finally, after a time $t \sim Q^{2} / M^{3}$, can be identified as a proton. What we wish to study here is exactly how the expansion from a compact three quark system to a physical proton takes place 12 . 


\subsubsection{Classical Expansion}

We begin by considering a model where the compact quark system expands to a size of one fermi by free motion. In order to simplify the discussion we shall consider the expansion of a quark-antiquark system having $\Delta \vec{x} \approx 0$ to its final configuration $\Delta x_{\perp} \sim 1 \mathrm{fm}, \Delta z=1 / p$. The situation is illustrated in Fig. 3.5. At the final configuration, $\Delta x_{\perp} \sim 1 \mathrm{fm}$, the quark-antiquark fit a normal pion wavefunction and the expansion ceases.

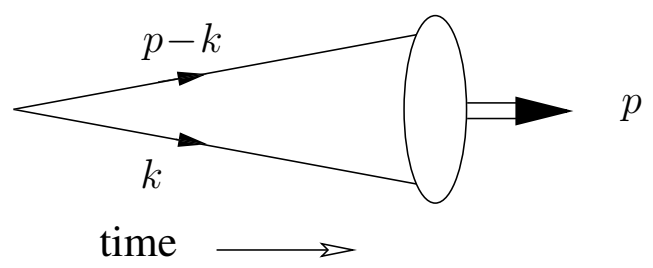

Figure 3.5: The expansion from a point-like configuration to a physical pion.

We choose $p_{\mu}=\left(E_{p}, 0,0, p\right)$ and we suppose that $k_{\perp} \approx \kappa=\sqrt{\left\langle k_{\perp}^{2}\right\rangle}$ the average transverse momentum of a pion while $k_{z}=x p$ with $0<x<1$ and $x$ taking on a typical value away from the endpoints at 0 and 1 . We choose time $t=0$ as the starting time of the expansion. Let us call the quark (or antiquark) $k$ the 1 particle and the quark $(p-k)$ the 2 particle. The transverse velocity of the two particles are

$$
\begin{aligned}
v_{1 \perp} & =\frac{k_{\perp}}{k_{z}}=\frac{\kappa}{x p} \\
\text { and } \quad & v_{2 \perp}=\frac{-k_{\perp}}{(p-k) z}=-\frac{\kappa}{(1-x) p} .
\end{aligned}
$$


Then the relative transverse velocity is

$$
v_{\perp}=\left|v_{1 \perp}-v_{2 \perp}\right|=\frac{\kappa}{p x(1-x)} .
$$

After a time $t$ the transverse separation of the quark-antiquark pair is

$$
\Delta x_{\perp}=v_{\perp} t=\frac{\kappa t}{p x(1-x)} .
$$

When $\Delta x_{\perp}=2 r_{0}$, the radius of the pion, the expansion should stop, and at that time a normal pion can be said to be formed. This formation time, $t_{F}$, is given by

$$
t_{F} \approx \frac{1}{2} \frac{r_{0} p}{\kappa},
$$

where we have set $x=1 / 2$ to get a typical time. If $r_{0} \approx 1 \mathrm{fm}$ and $\kappa \approx 350 \mathrm{MeV}$ then

$$
t_{F} \approx \frac{3}{4} p
$$

with $t_{F}$ expressed in fermis and $p$ in $\mathrm{GeV}$. A similar result holds for a three quark system expanding to form a proton. If one assumes that three quarks share the longitudinal momentum equally then

$$
t_{F}=\frac{1}{3} \frac{r_{0} p}{\kappa}
$$

with $p$ the momentum of the proton, $\kappa$ the typical transverse momentum of a quark in the proton and $r_{0}$ the radius of the proton.

As an expanding proton moves through the nucleus it should interact with the nucleons of the nucleus with a cross section proportional to $\pi r_{\perp}^{2}(t)$ where 
$r_{\perp}$ is the transverse radius of the three quark system. Since $r_{\perp}(t)$ is linear in $t$ the three quark system should have a cross section with nucleons

$$
\sigma(t)=\sigma\left(\frac{t}{t_{F}}\right)^{2}
$$

which cross section can be used to calculate the rate at which inelastic reactions occur. $\sigma_{0}$ in (3.17) is a normal nucleon-nucleon cross section. Thus in order for quasielastic reactions to be likely it is necessary that $\sigma_{0}\left(R / t_{F}\right)^{2}$ be small with $R$ the radius of the nucleus. Of course at sufficiently high energy $t_{F}$ is very large and the above condition can, in principle, be satisfied.

\subsubsection{Quantum Expansion}

The picture of expansion from a compact quark state to a normal part of a wavefunction of a hadron that we presented in the last section is intuitively appealing, but it cannot really be correct. The uncertainty principle in quantum mechanics requires that a compact state have high transverse momentum components while we have assumed that $k_{\perp}$ could be replaced by a parameter $\kappa$ fixed in terms of the normal wavefunction of th hadron. In this section we shall derive a quantum expansion formula based on the evolution of the quark system in QCD. For simplicity we shall again deal with a quark-antiquark expanding towards a normal configuration of the wavefunction of the pion.

The evolution of a quark-antiquark pair from a radius $r_{i}$ to a final radius $r_{0}$, with $r_{i} / r_{0} \ll 1$ and $r_{0}$ the radius of the pion, is governed by the QCD graphs shown in Fig. [3.6, where $k_{1 \perp}^{2} \approx 1 / r_{i}^{2}$ is the starting point for the evolution. The graphs in Fig. 3.6, when evaluated in light-cone gauge, give the expansion correctly at the leading logarithmic level of approximation. 


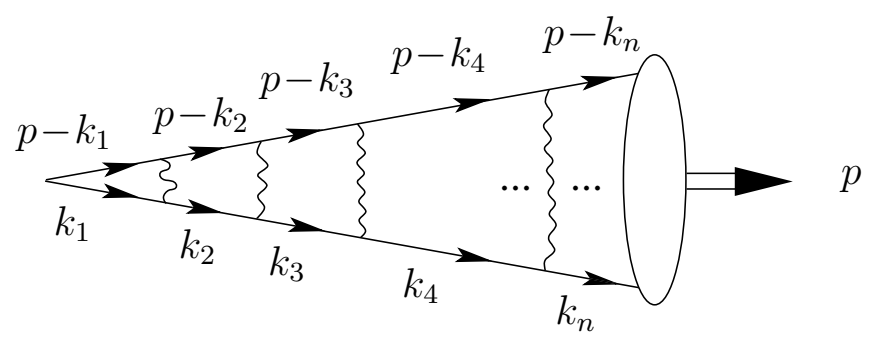

Figure 3.6: The dynamics of the expansion of the pion's wavefunction.

The lifetime of the $k_{1}-\left(p-k_{1}\right)$ quark-antiquark pair is given by

$$
\tau_{1}^{-1}=\frac{k_{1 \perp}^{2}}{2\left(p-k_{1}\right) z}+\frac{k_{1 \perp}^{2}}{2 k_{1 z}}=\frac{k_{1 \perp}^{2}}{2 p x(1-x)},
$$

where $k_{1 z}=x_{1} p_{1}$. Over the time $\tau_{1}$ the quark-antiquark pair separate a distance

$$
\Delta x_{1 \perp}=v_{1 \perp} \tau_{1} .
$$

Using

$$
v_{1 \perp}=\frac{k_{1 \perp}}{p x_{1}\left(1-x_{1}\right)}
$$

one obtains

$$
\Delta x_{1 \perp}=\frac{2}{k_{1 \perp}}
$$

a result which agrees with uncertainty principle. Similarly the lifetime of the state $k_{n}-\left(p-k_{n}\right)$ and the separation during that period of time are given by

$$
\tau_{n}^{-1}=\frac{k_{n \perp}^{2}}{2 p x_{n}\left(1-x_{n}\right)}
$$


and

$$
\Delta x_{n \perp}=\frac{2}{k_{n \perp}} .
$$

Because of the weakness of the coupling in QCD the $k_{i \perp}$ obey $k_{1 \perp}^{2} \gg k_{2 \perp}^{2} \gg$ $\ldots \gg k_{n \perp}^{2} \gg \ldots$ Thus after $n$ gluon exchanges $t$ and $\Delta x_{\perp}$ are given by (3.20) and (3.21) to a good approximation. Using (3.21) in(3.20) one obtains

$$
r_{\perp}^{2}(t)=\left[\frac{\Delta x_{\perp}(t)}{2}\right]^{2} \approx \frac{2 t}{p},
$$

where we have set $x(1-x)=1 / 4$ to get an average value of $r_{\perp}^{2}(t)$. This leads to a cross section with nucleons in the nucleus of

$$
\sigma(t)=\sigma_{0}\left(t / t_{F}\right)
$$

in contrast to (3.17). Thus in the quantum expansion picture $\sigma(t)$ grows linearly with $t$ whereas in a classical expansion it grows quadratically. (3.17) is a result corresponding to the naive parton model while (3.23) is the correct QCD formula for the expansion of a newly born quark system as it moves to a more normal hadronic configuration. For $r_{i}$ very small and for $p$ very large (3.23) is clearly the correct expression. However, for exclusive reactions which are not at truly asymptotic energies it is difficult to say that (3.23) is a more accurate formula than (3.17). A good, complete experimental program studying almost exclusive reactions in nuclei should be able to tell us which is the better formula at a given momentum transfer. 


\section{Bibliography}

1. For a review see S.J. Brodsky and G.P. Lepage. In Perturbative Quantum Chromodynamics, ed. A.H.Mueller, World Scientific, Singapore, 1989.

2. V.A. Matveev, R.M. Muradyan and A.N. Tavkhelidze. Lett. Nuovo Cimento, 7:719, 1973.

3. S.J. Brodsky and G. Farrar. Phys. Rev. Lett., 31:1153, 1973.

4. P.V. Landshoff. Phys. Rev., D10:1024, 1974.

5. A.H. Mueller. Phys. Rep., 73:237 1981.

6. J. Botts and G. Sterman. Nucl. Phys., B325:62, 1989.

7. V.L. Chernyak and I.R. Zhitnitskii. Phys. Rep., 112:1783, 1984.

8. I.D. King and C.T. Sachrajda. Nucl. Phys., B279:785, 1987.

9. N. Isgur and C.H. Llewellyn Smith. Nucl. Phys., B317:526, 1989.

10. A.H. Mueller. In Proceedings of the $17^{\text {th }}$ Recontre de Moriond, ed. J.Tran Thanh Van, Editions Frontieres, Gif-sur-Yvette, 1982.

11. G. Bertsch, S.J. Brodsky, A.S. Goldhaber and J.F. Gunion. Phys. Rev. Lett., 47:297, 1981.

12. G. Farrar, H. Liu, L. Frankfurt and M. Strikman. Phys. Rev. Lett., 61: 686, 1988.

13. A.S. Carroll et al.. Phys. Rev. Lett., 61:1698, 1988.

14. B. Pire and J. Ralston. Phys. Rev. Lett., 61:1823, 1988. 


\section{Chapter 4}

\section{Guide to Color Coherence}

\section{Contents}

4.1 Angular Ordered Parton Cascades . . . . . . . . 114

4.2 Angular Ordering in Initial State Radiation . . . 117

4.3 The Origin of Hump-Backed QCD Plateau . . . . 123

4.4 Radiation pattern for the $q \bar{q}$ Antenna . . . . 126

4.5 Wave Nature of Drag Effects . . . . . . . . 129

4.6 Two-particle Correlations . . . . . . . . . 132

4.7 Fevnman-Gribov Puzzle and QCD Coherence . . 134

Bibliography .................... 140

During recent years lots of discussions has been provoked by the theoretical prediction of coherent color emission and its experimental study. Moreover 
the experimental confirmation of the bright coherence phenomena has played an important role in the recognition of the whole of the perturbative approach to jet physics. Our aim in this Chapter is to provide the reader with Fingerspitzgefühl of the basic coherence phenomena (see Refs. 112). We hope that this Chapter will be useful for anyone who wants to understand the origin and the essence of color coherence effects.

Coherence problems are basic to any gauge theory. Roughly speaking there are two types of coherence phenomena which occur in QCD jet dynamics: intRAjet and intERjet coherence.

The first manifestation of intrajet coherence is the angular ordering (AO) of the sequential parton decays. AO accounts for the appropriate dependence of the soft gluon radiation on the prehistory of parton jet development.

Interjet coherence deals with the angular structure of soft particle flows when three or more energetic partons are involved in a hard process. Here the

particle angular distributions depend on the geometry and color topology of the whole jet ensemble giving rise to QCD radiophysics of jets (see Chapter 9).

\subsection{Angular Ordered Parton Cascades}

To elucidate the physical origin of AO let us consider a simple model of the jet cascade, namely, the radiation pattern of soft photons produced by a relativistic $e^{+} e^{-}$pair in a QED shower (see Fig. 4.1).

The question is to what extent the $e^{+}$and $e^{-}$independently emit $\gamma^{\prime}$ s. To answer this question let us estimate the formation time, the time interval needed for the $\gamma$-quantum to be radiated from, say, the $e^{-}$leg. Using the uncertainty relation to estimate the "lifetime" of the electron ( $c f$. (1.7)) one 


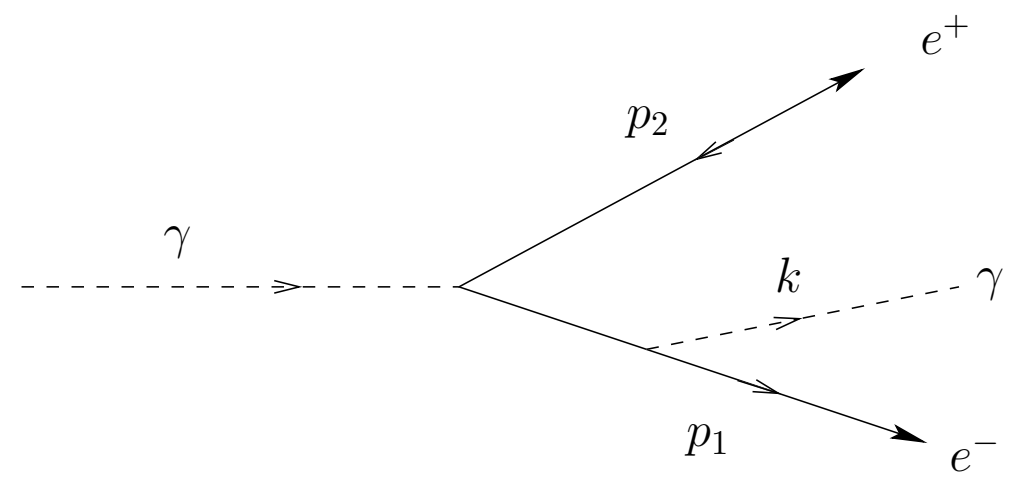

Figure 4.1: Bremsstrahlung radiation of a photon $\quad k \quad$ after $e^{+} e^{-}$pair production.

finds

$$
t_{\text {form }} \approx \frac{1}{M_{\text {virt }}} \frac{p_{e}}{M_{\text {virt }}} \approx \frac{1}{k \Theta_{\gamma e}^{2}},
$$

where $\Theta_{\gamma e}$ is the angle between the emitted photon and the electron. Now $k \Theta_{\gamma e^{-}}=k_{\perp}=\lambda_{\perp}^{-1}$ with $\lambda_{\perp}$ standing for the transverse wavelength of the radiated photon. So one can rewrite (4.1) as

$$
t_{\text {form }} \approx \lambda_{\perp} / \Theta_{\gamma e^{-}} .
$$

During this period of time the $e^{+} e^{-}$pair separates, transversely, a distance

$$
\rho_{\perp}^{e^{+} e^{-}} \approx \Theta_{e^{+} e^{-}} t_{\text {form }} \approx \lambda_{\perp} \frac{\Theta_{e^{+} e^{-}}}{\Theta_{\gamma e}} .
$$

One concludes that for large angle photon emissions,

$$
\Theta_{\gamma e^{-}} \approx \Theta_{\gamma e^{+}} \gg \Theta_{e^{+} e^{-}},
$$


the separation of two emitters, $e^{+}$and $e^{-}$, proves to be smaller than $\lambda_{\perp}$. In this case the emitted photon cannot resolve the internal structure of the $e^{+} e^{-}$pair and probes only its total electric charge, which is zero. Thus for $\Theta_{\gamma e^{-}} \gg \Theta_{e^{+} e^{-}}$we expect $\gamma$ to be strongly suppressed. 11 The $e^{+}$and $e^{-}$can be said to emit photons independently only at $\rho_{\perp}^{e^{+}} e^{-}>\lambda_{\perp}$, that is when

$$
\Theta_{\gamma e^{-}}<\Theta_{e^{+} e^{-}} \text {or } \Theta_{\gamma e^{+}}<\Theta_{e^{+} e^{-}} .
$$

A similar physical picture can be drawn for QCD cascades where soft gluon radiation is governed by the conserved color currents. The only difference is that the coherent radiation of soft gluons by an unresolved pair of quarks is no longer zero but the radiation acts as if it were emitted from the parent gluon imagined to be on shell, as is illustrated in Fig. 4.2.

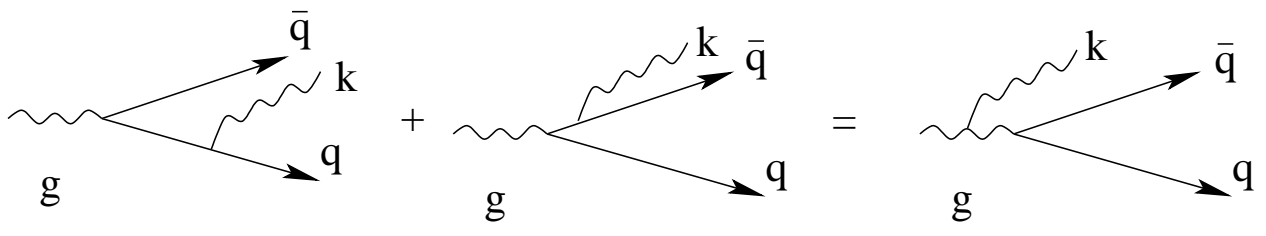

Figure 4.2: Wide-angle emission of soft gluon $k$, off $q$ and $\bar{q}$, acts as if the emission came off the parent gluon $g$ imagined to be on shell.

This property is universal and holds true for the soft radiation accompanying $q \rightarrow q g$ and $g \rightarrow g g$ splittings as well. The remarkable fact is that one gets not only all leading double logarithmic but (as we shall see later

\footnotetext{
${ }^{1}$ This phenomenon has been well known in cosmic ray physics from the middle of fifties — the so called "Chudakov effect" [3].
} 
in Chapter [6) also nonleading single logarithmic effects correctly, for angular averaged observables 4 , by allowing the gluon emission, independently,

off line $\bar{q}$ when $\quad \Theta_{k \bar{q}} \leq \Theta_{q \bar{q}}$,

off line $q$ when $\quad \Theta_{k q} \leq \Theta_{q \bar{q}}$,

and off the parent, line $g$, when $\quad \Theta_{k g} \geq \Theta_{q \bar{q}}$.

This observation furnishes the core idea of the Marchesini-Webber model 5 the first Monte Carlo simulation that included intrajet coherence effects. At the moment this strict AO prescription is incorporated into the main Monte Carlo shower algorithmic models (for reviews see Ref. [6]).

The yield of soft, wider-angle gluons remains unchanged when the number of particles inside the multipartonic bunch increases, since such gluons are unable to resolve separate color sources inside the parton jet. The soft gluon behaves as a classical probe testing the color charge of the jet as a whole, i.e. that of the original parton, initiating the jet. In this sense QCD color coherence can be said to suppress soft radiation at large angles. As a result, to describe the jet evolution in terms of independent sequential parton splittings one has to impose the AO condition - a uniform decrease of successive opening angles in the cascade (Fig. 4.3).

\subsection{Angular Ordering in Initial State Radiation}

Bremsstrahlung originating from the space-like cascades is usually called initial state radiation due to the fact that it is the presence of incoming color fields which causes the partonic wave function of the target nucleon to be formed long before the instant of the hard interaction. Space-like development of partonic systems determines the target fragmentation in deeply 


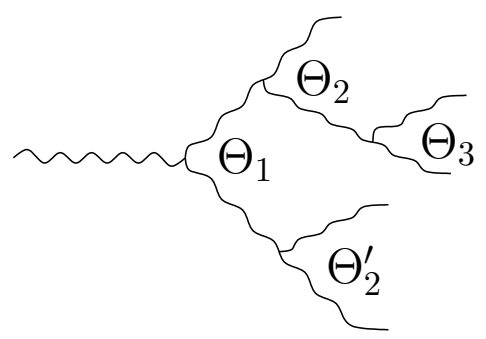

Figure 4.3: Angular Ordering in the cascade : $\Theta_{1}>\Theta_{2}>\Theta_{3} \ldots, \Theta_{1}>\Theta_{2}^{\prime} \ldots$

inelastic lepton-hadron scattering (DIS), structure of final hadron states in Drell-Yan and high- $p_{\perp}$ scattering processes etc., see Refs. [7-9]. Gluon radiation off initial (space-like) and final (time-like) partons can be separated, provided that interference between emission amplitudes has been taken into account, and a probabilistic scheme constructed. It proves to be AO that provides such a probabilistic interpretation.

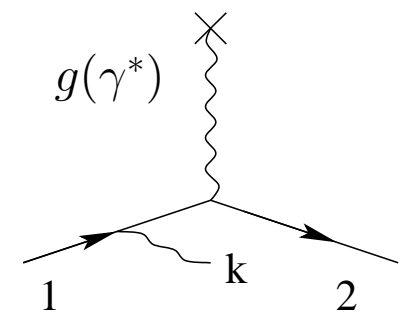

(a)

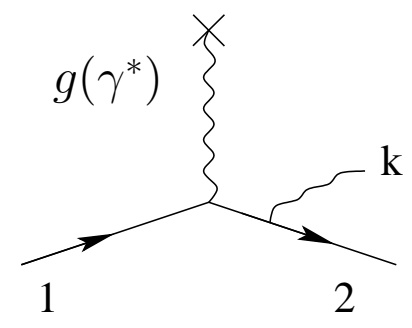

(b)

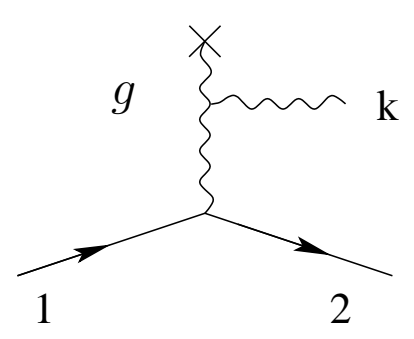

(c)

Figure 4.4: Soft gluon radiation in the parton scattering process with (and without) color transfer in $t$-channel. 
Consider, firstly, the soft gluon radiation in the case of an energetic parton scattering at finite angle $\Theta_{s}$, when in the $t$-channel color is not transferred (e.g., electroweak quark scattering). As well known one should observe here two bremsstrahlung cones with opening angles $\Delta \Theta \approx \Theta_{s}$ centered in the directions of incoming and scattered partons $\left(\Theta_{1 k}<\Theta_{s}, \Theta_{2 k}<\Theta_{s}\right.$, with $\Theta_{s} \equiv$ $\Theta_{12}$ the scattering angle, see Figs 4.4a,b).

Soft emission at large angles $\Theta \equiv \Theta_{1 k} \sim \Theta_{2 k}>\Theta_{s}$ is absent since during the time $t_{\text {form }}=\lambda_{\perp} / \Theta$ the transverse displacement of the charge proves to be small: $\rho_{\perp} \approx \Theta_{s} t_{\text {form }}<\lambda_{\perp}$, and the situation looks like there were no change of the current at all.

For scattering with color transfer in $t$-channel the additional bremsstrahlung contribution appears, which corresponds effectively to the emission off the $t$-channel gluon, when $\Theta_{1 k} \approx \Theta_{2 k}>\Theta_{s}$, see Fig. 4.4k. In spite of negligible transverse displacement, the color current of the quark has been affected here by the gluon exchange.

\section{Accompanying particle distribution in the heavy Higgs production} should be mentioned as an instructive pedagogical example 10. Here the radiation pattern should be quite different depending on the production mechanism of a Higgs boson, see Fig. 4.5. In the case of the $W-W$ production mechanism of Fig. 4.5 the central region in rapidity distribution corresponding to large angles $\left(\Theta>\Theta_{s} \sim M_{H} / E\right)$ is depopulated with particles and the process looks like the quasi-diffractive one (the gluon emission by initial and final partons at large angles cancel coherently). Meanwhile, for $H$ boson production via $g-g$ fusion of Fig.4.5b the standard uniform rapidity plateau with the particle density corresponding to the "gluon jet" emerges in the hadronic spectrum, see Chapter 9 . 


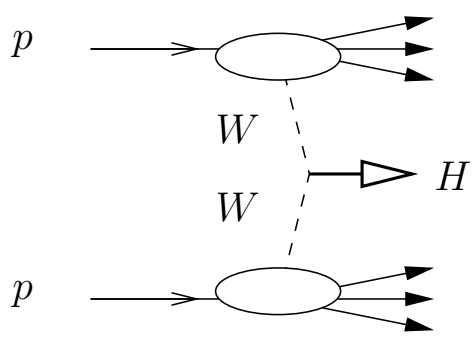

(a)

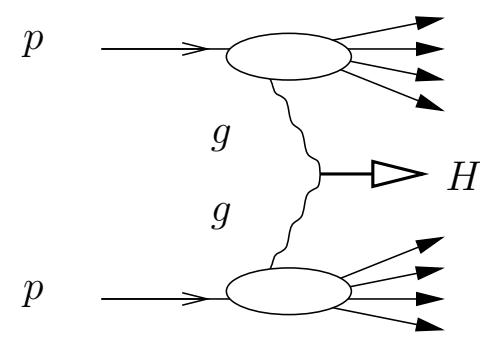

(b)

Figure 4.5: Hadronic Higgs boson production via (a) $W W$ fusion and (b) gluon-gluon fusion.

Heavy quark production gives another interesting example of initial state coherence. It is the large quark mass $m_{Q} \gg \Lambda$ that determines specific features of jets induced by $Q$. With account of the $Q$ mass and width 2 the probability of gluon radiation off $Q$ with momentum $p=(E, \vec{p})$ is proportional to the square of the virtual quark propagator shown by a thick line in Fig.4.6.

$$
d w \propto\left|\frac{1}{(p+k)^{2}-\left(m_{Q}-i \frac{\Gamma_{Q}}{2}\right)^{2}}\right|^{2}
$$

and for the case of soft quasi-collinear gluon $k=(\omega, \vec{k}), \quad k_{\perp} \ll \omega \ll E$, takes the form

$$
d w \approx \frac{\alpha_{s}}{\pi} C_{F} \frac{k_{\perp}^{2} d k_{\perp}^{2}}{\left[k_{\perp}^{2}+\left(\omega m_{Q} / E\right)^{2}\right]^{2}+\left(\omega m_{Q} \Gamma_{Q} / E\right)^{2}} \frac{d \omega}{\omega} .
$$

\footnotetext{
${ }^{2} \Gamma_{Q}$ can well happen to be an extra large parameter for the sufficiently heavy top-quark
} 


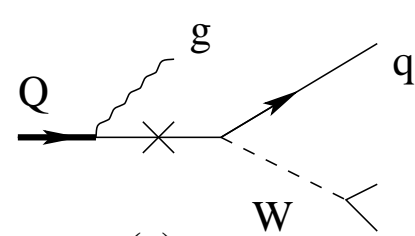

(a)

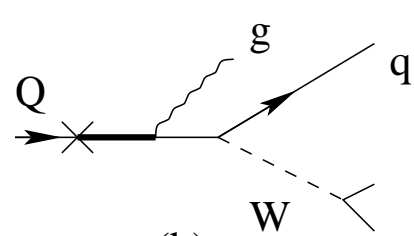

(b)

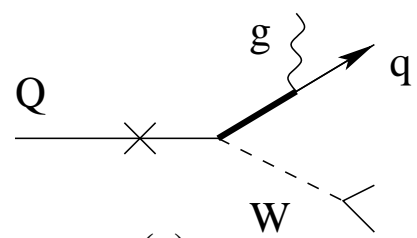

(c)

Figure 4.6: Soft gluon emission accompanying heavy quark production.

The usual double logarithmic radiative pattern is obtained from (4.4) only for sufficiently large transverse momenta:

$$
\begin{aligned}
k_{\perp}^{2} & >\left(\frac{\omega}{E} m_{Q}\right)^{2}, \\
\text { or } \quad \Theta^{2} & >\Theta_{0}^{2}, \quad \Theta_{0} \equiv \frac{m_{Q}}{E} .
\end{aligned}
$$

Moreover, with gluon energy $\omega$ taken small enough, additional restriction of forward emission emerges due to finite value of $\Gamma_{Q}$, so that

$$
\begin{aligned}
k_{\perp}^{2} & >\frac{\omega}{E} m_{q} \Gamma_{Q} \quad \text { for } \quad \omega<\frac{E}{m_{Q}} \Gamma_{Q}, \\
\text { or } \quad \Theta^{2} & >\frac{m_{Q} \Gamma_{Q}}{E \omega} .
\end{aligned}
$$

The last condition could be easily understood in terms of the gluon formation time which has to be less than the $Q$-lifetime in the laboratory frame.

Looking at (4.5), (4.6) one might naively expect that the measured bremsstrahlung particle distributions are sensitive to the heavy quark parameters, in particular, to $\Gamma_{Q}$. This possibility, however, proves to be illusive just because 
of QCD coherence. When studying the inclusive radiation pattern one has to take into account gluon emissions off the final state, depicted in Fig. 4.6b,c. With the color of $Q$ being transferred to the light (lighter) quark $q$ after the weak $Q$-decay, the whole radiation pattern appears to be insensitive to quark parameters $m_{Q}$ and $\Gamma_{Q}$. Indeed, the weak decay producing a relativistic $q$ is accompanied by QCD bremsstrahlung that corresponds to a hard process with effective hardness $\sim m_{Q}^{2}$. In the laboratory frame these particles fill in precisely the "Dead Cone" $\Theta \lesssim \Theta_{0}$ (see (4.5b) thus "screening" the mass dependence. There are no particles emitted off the final quark at angles larger than $\Theta_{0}$ because of destructive interference between amplitudes of Fig. 4.6 b and 4.6k. This cancellation works exactly as in the case of quark scattering without color transfer discussed above (with colorless $W$ playing the role of "scattering potential" from Fig. 4.4a).

Additional suppression of soft quasi-collinear emissions given by (4.6) has two consequences. First of all, it makes the "Dead Cone" increasing with decrease of gluon energy according to (4.6b). But at the same time it destroys the complete cancellation between (b) and (c) amplitudes since the "real" $Q$ quark in Fig. 4.6b simply has no appropriate gluon field components to interfere with the radiation off the $q$-line at $\omega<\Gamma_{Q} E / m_{Q}, \Theta_{0}<\Theta<\sqrt{E m_{Q} / \omega \Gamma_{Q}}$. This enlarges the yield of bremsstrahlung particles produced along with the $Q$-decay process by an amount which is necessary to compensate exactly the lack of particles from the $Q$-production stage of Fig. 4.4 . Thus, no sign of either $\Gamma_{Q}$ or $m_{Q}$ effects is left in the structure of QCD bremsstrahlung itself. 


\subsection{The Origin of Hump-Backed QCD Plateau}

The depletion of soft emission leads to the "hump-backed plateau" in the inclusive energy spectrum of particles inside jet, which is one of the most striking predictions of perturbative QCD. This phenomenon can be understood on kinematical grounds as the result of two conflicting tendencies: on one hand, owing to the restriction $k_{\perp}>1 / R$ a soft particle is "forced out" at large emission angles $\Theta>1 / k R$, on the other hand, the allowed decaying angle is shrunk to small values after a few successive AO parton branching.

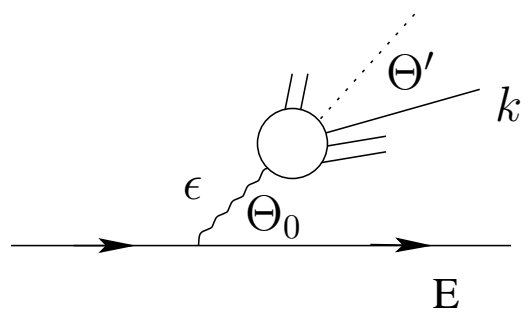

Figure 4.7: First order "toy"-model for illustrating the origin of hump-backed plateau.

Let us illustrate the influence of the color coherence on particle spectra with the help of the toy model 11 for parton branching, based on the first order QCD, as shown in Fig. 4.7. We start with an old-fashioned plateau $\rho(k) \equiv d n / d \ln k=$ const of hadrons with limited transverse momenta $k \Theta=k_{\perp} \sim R^{-1}$ for a quark jet with energy $E$ (see shaded area in Fig. 4.8). Accounting for a gluon with energy $\epsilon$ and emission angle $\Theta_{0}$, let us 


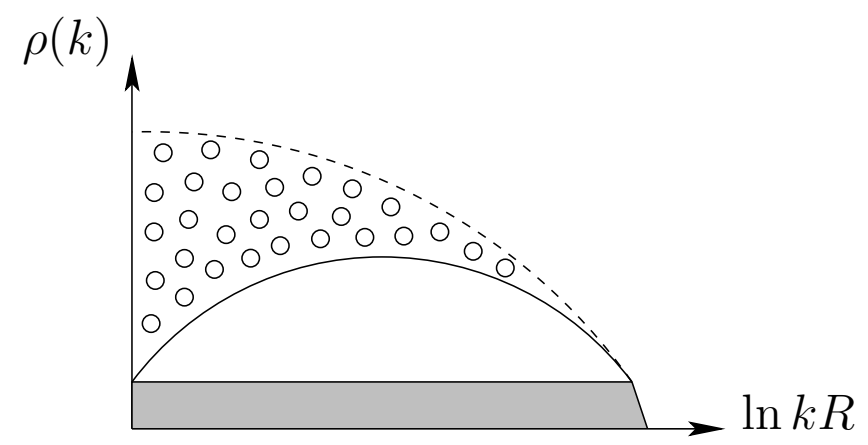

Figure 4.8: The effect of color coherence on particle energy spectrum $\rho(k)=d n / d \ln k$. Dotted area corresponds to the contribution which is removed when turning from the incoherent model (dashed) to the coherent one ( solid). Shaded area shows the old-fashioned plateau (without taking account of bremsstrahlung).

use the double-logarithmic expression for the radiation probability

$$
d w_{g} \propto \alpha_{s} \frac{d \epsilon}{\epsilon} \frac{d \Theta_{0}}{\Theta_{0}} \vartheta\left(\epsilon \Theta_{0}-R^{-1}\right) .
$$

The step function $\vartheta$ restricts here the transverse momentum $p_{\perp} \approx \epsilon \Theta_{0}>R^{-1}$ to ensure the gluon's being:

$$
t_{\text {form }} \approx \frac{\epsilon}{p_{\perp}^{2}}<t_{\text {hadr }} \approx \epsilon R^{2} .
$$

How does the gluon contribute to the particle yield? From the standpoint of the orthodox parton model one might expect the gluon to give rise to its own plateau of particles with energies

$$
R^{-1}<k<\epsilon
$$


and limited transverse momenta with respect to the gluon: $k \Theta^{\prime} \sim R^{-1}$ (see Fig. 4.7).

Now let us verify that the QCD coherence leads to the following reduction of this additional plateau:

$$
\left(R \Theta_{0}\right)^{-1}<k<\epsilon
$$

The plateau distribution of particles from the gluon jet can be represented symbolically as

$$
d N=\frac{d k}{k} \frac{d \Theta^{\prime}}{\Theta^{\prime}} \delta\left(k \Theta^{\prime}-R^{-1}\right) .
$$

This expression can be thought of as a double logarithmic spectrum of bremsstrahlung from a gluon $\left(\epsilon, \Theta_{0}\right)$ "projected" onto the domain of the most intensive radiation $\left(\alpha_{s}\left(k \Theta^{\prime}\right) / \pi \sim 1\right)$. As it follows from the $\mathrm{AO}$ in cascade, the offspring particles are independently emitted by the gluon only inside the cone with the opening angle $\Theta^{\prime}<\Theta_{0}$. Applying this inequality to (4.9), one obtains restriction (4.8) at once.

The condition (4.8) reflects the fact that the bremsstrahlung particle yield depends rather on $p_{\perp} \approx \epsilon \Theta_{0}$ of a parent gluon than on its energy $\epsilon$. Finally, our toy model gives us the following particle multiplicity:

$$
\begin{aligned}
N & =\int^{E} \frac{d k}{k} \int^{1} \frac{d \Theta}{\Theta} \delta\left(k \Theta-R^{-1}\right) \\
& +\alpha_{s} \int^{E} \frac{d \epsilon}{\epsilon} \int^{1} \frac{d \Theta_{0}}{\Theta_{0}} \vartheta\left(\epsilon \Theta_{0}-R^{-1}\right) \int^{\epsilon} \frac{d k}{k} \int^{\Theta_{\max }} \frac{d \Theta^{\prime}}{\Theta^{\prime}} \delta\left(k \Theta^{\prime}-R^{-1}\right) .
\end{aligned}
$$

The first term of this schematic expression stands for the background quark plateau, the second one is constructed from the gluon emission (4.7) and 
fragmentation (4.9). The parameter $\Theta_{\max }$ encodes the difference between the coherent and incoherent cases:

$$
\begin{aligned}
\Theta_{\max }^{\text {incoh. }} & =1, \\
\Theta_{\max }^{c o h} & =\Theta_{0} .
\end{aligned}
$$

Using (4.10) one obtains for the particle energy spectrum $\rho(k)$

$$
\begin{aligned}
\rho^{\text {inco }} & =1+\frac{\alpha_{s}}{2}\left(\ln ^{2} E R-\ln ^{2} k R\right), \\
\rho^{c o h} & =1+\alpha_{s} \ln \frac{E}{k} \ln k R .
\end{aligned}
$$

The additional gluon-initiated multiplicity $\int d \ln k(\rho(k)-1)$ appears to be twice as large for the incoherent case (this factor 2 exponentiates with account of multiple branching, see Chapter (5).

Expressions (4.11) illustrate qualitatively the bright fact that the coherence substantially depletes the soft part of the energy spectrum giving rise to a hump (see Fig. 4.8). The height of the hump is increasing with energy and peaks at $k \sim \sqrt{E}$. It is noteworthy that the incoherent monotonic spectrum (4.11a) would also have a peak due to kinematical mass effects $\left(\rho(k) \propto \sqrt{k^{2}-m^{2}}\right.$ at small energies $\left.k \approx m\right)$ but placed near the phase space limit $k \sim m \sim R^{-1}$.

\subsection{Radiation pattern for the $q \bar{q}$ Antenna}

Here we shall examine soft gluon emission associated with $q \bar{q}$ pair produced in a color-singlet state in some hard process (see Refs. [12 13]). This radiation pattern is interesting in its own right, e.g., in connection with two-jet physics in the process $e^{+} e^{-} \rightarrow q \bar{q}$. Furthermore, neglecting the terms of the order 
$1 / N_{c}^{2}$, one can represent the radiation pattern in the case of an arbitrary complex hard partonic system as a sum of terms in which each external quark line is uniquely connected to an external antiquark line of the same color (colorless " $q \bar{q}$-antennae").
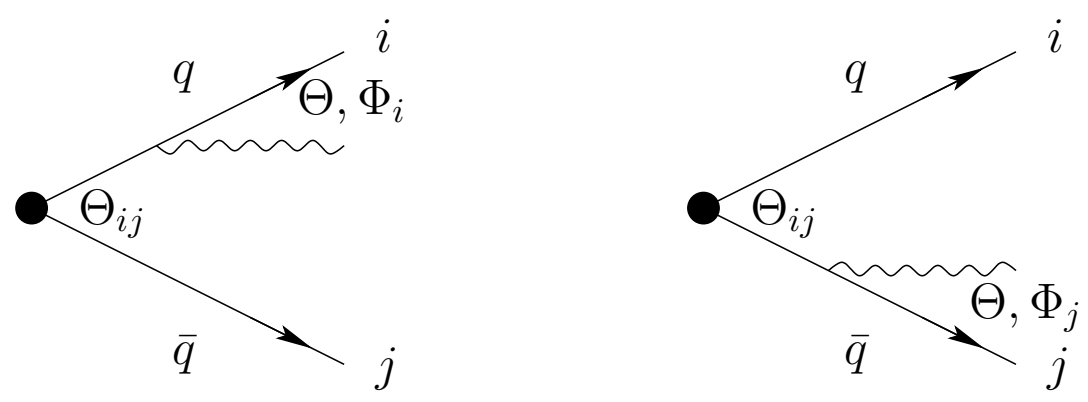

Figure 4.9: Soft gluon emission off a hard colorless $q \bar{q}$ pair.

In lowest order the soft gluon distribution takes the familiar form (for notations see Fig. 4.9)

$$
d w_{q \bar{q}}=-\frac{d^{3} k}{(2 \pi)^{2} k} \alpha_{s} C_{F}\left(\frac{p_{i}}{\left(p_{i} \cdot k\right)}-\frac{p_{j}}{\left(p_{j} \cdot k\right)}\right)^{2}=\frac{d k}{k} d \Omega_{\vec{n}} \frac{\alpha_{s}}{(2 \pi)^{2}} W^{q \bar{q}}(\vec{n}) .
$$

Here

$$
W^{q \bar{q}}(\vec{n})=2 C_{F} \widehat{(i j)}, \quad \widehat{(i j)}=\frac{a_{i j}}{a_{i} a_{j}} ; \quad a_{i j}=\left(1-\vec{n}_{i} \vec{n}_{j}\right), \quad a_{i}=\left(1-\vec{n} \vec{n}_{i}\right),
$$

$\vec{n}_{i}, \vec{n}_{j}$ denote the directions of $q, \bar{q}$ momenta respectively; $\vec{n}$ is the direction of the emitted gluon.

Let us call the distribution $\widehat{(i j)}$, describing the radiation pattern of the colorless $q \bar{q}$ pair, the "q $q$-antenna". The antenna $\widehat{(i j)}$ may be represented in 
the form

$$
\begin{aligned}
\widehat{(i j)} & =P_{i j}+P_{j i}, \\
P_{i j} & =\frac{1}{2}\left[\frac{1}{a_{i}}+\frac{a_{i j}-a_{i}}{a_{i} a_{j}}\right] .
\end{aligned}
$$

One can refer to the two terms in the square bracket in 4.14b) as the incoherent and the interference terms. At fixed $\Theta_{i}$, the incoherent term is independent of the azimuthal angle $\phi_{i}$. The interference term depends on $\phi_{i}$ through the angle $\Theta_{j}$. If the soft gluon lies within the cone, determined by $i$ and $j$, the interference is positive. If the gluon lies outside this cone the interference is negative. This term is largest when the soft gluon lies in the plane defined by $i$ and $j, \phi_{i}=\phi_{j}$. The point about splitting the radiation pattern into two terms $P_{i j}$ and $P_{j i}$ is that only the former (latter) has the pole at $\Theta_{i}=0\left(\Theta_{j}=0\right)$, so this term can be treated as "belonging to" quark $i(j)$.

After integration over $\phi_{i}$ the contributions of the incoherent and interference terms turn out to be equal in magnitude having opposite signs outside the parent cone, so that

$$
\left\langle P_{i j}\right\rangle_{\phi_{i}} \equiv \int \frac{d \phi_{i}}{2 \pi} P_{i j}(\vec{n})=\frac{1}{a_{i}} \vartheta\left(\cos \Theta_{i}-\cos \Theta_{i j}\right) .
$$

In other words, $\left\langle P_{i j}\right\rangle_{\phi_{i}}$ is just the incoherent radiation $P_{i}^{\text {incoh }}$ off a quark $i$, effectively confined to the cone

$$
\cos \Theta_{i} \geq \cos \Theta_{i j}, \quad \Theta_{i} \leq \Theta_{i j} .
$$

This result allows one to incorporate soft gluon interference effects in the Monte Carlo simulations of partonic cascades in a probabilistic way. For the 
case of multiparton system with $n$ hard emitters $(i=1 \ldots n)$ the full soft radiation pattern $P(\Omega)$ may be replaced by

$$
P(\Omega) \Longrightarrow P_{A O} \equiv \sum_{i}^{n} P_{i}^{i n c o h .} \vartheta\left(-\Theta_{i}+\Theta_{i j}\right),
$$

which looks as a sum of independent emission probabilities.

\subsection{Wave Nature of Drag Effects}

The drag (or string) effect in the $q \bar{q} g$ events of $e^{+} e^{-}$annihilation is one of the best examples of QCD coherence of the second kind. In Chapter 9 we shall have much more to say about this phenomenon, however, our purpose here is simply to explain the basic idea. So far, the most striking experimental test of drag effects is the comparison of associated hadron production in $q \bar{q} g$ three jet events with that of $q \bar{q} \gamma$ events with the $g$ and $\gamma$ having similar kinematics. In the plane of the three jets, counting the photon as a jet, one finds a suppression of associated hadrons in the region between the $q$ and $\bar{q}$ in $q \bar{q} g$ events as compared to $q \bar{q} \gamma$ events.

It might seem strange at the first sight that inclusion of an additional "emitter" (colored gluon replacing colorless photon) results in less QCD radiation in some direction. This is typical quantum-mechanical interference effect and one can illustrate its physical origin with a help of a "QED" model 1$]$ where quarks are replaced by electrons and the gluon - by a collinear $e^{+} e^{+}$ pair as shown in Fig. 4.10k. The $q \bar{q} \gamma$ event is illustrated in this model by 
Fig. 4.10 p. The corresponding radiation patterns are:

$$
\begin{aligned}
d w_{q \bar{q} \gamma} & =\frac{d k}{k} d \Omega_{\vec{n}} \frac{\alpha}{2 \pi^{2}} \widehat{(12)} \\
d w_{q \bar{q}} " \mathrm{~g} " & =-\frac{d k}{k} d \Omega_{\vec{n}} \frac{\alpha}{(2 \pi)^{2}}\left(\frac{p_{1}}{\left(p_{1} \cdot k\right)}+\frac{p_{2}}{\left(p_{2} \cdot k\right)}-\frac{2 p_{3}}{\left(p_{3} \cdot k\right)}\right)^{2} \\
& =\frac{d k}{k} d \Omega_{\vec{n}} \frac{\alpha}{\pi^{2}}\left(\widehat{(13)}+\widehat{(23)}-\frac{1}{2} \widehat{(12)}\right)
\end{aligned}
$$
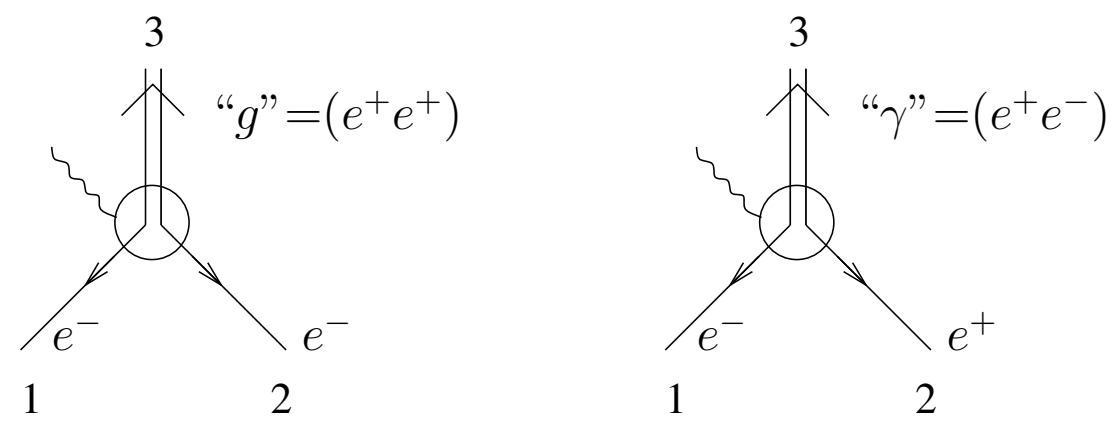

Figure 4.10: A "QED" model for illustrating drag effect. The gluon is represented as having double electric charge. The photon is replaced by collinear $e^{+} e^{-}$pair.

For notation see (4.13). Note that this pattern mimics the QCD $q \bar{q} g$ sample at $N_{c}=\sqrt{2}$, cf. (9.34).

For qualitative analysis it is convenient to rewrite the radiation pattern, given by (4.19), using the expression for the classical intensity of emission in 
terms of three-dimensional quantities (see, e.g., Ref. 14):

$$
\begin{aligned}
W^{q \bar{q} " g "}(\vec{n}) & =-\left(\frac{p_{1}}{\left(p_{1} \cdot k\right)}+\frac{p_{2}}{\left(p_{2} \cdot k\right)}-\frac{2 p_{3}}{\left(p_{3} \cdot k\right)}\right)^{2}=(\vec{A} \times \vec{n})^{2} ; \\
\vec{A} & =\left(\frac{\vec{n}_{1}}{a_{1}}+\frac{\vec{n}_{2}}{a_{2}}-\frac{2 \vec{n}_{3}}{a_{3}}\right) .
\end{aligned}
$$

One can easily see that for a symmetric configuration $\left(a_{13}=a_{23}\right)$ there is no radiation emitted directly opposite to the double charge: $\vec{A} \| \vec{n}$. This can be understood as the vanishing of the electric field midway between the equal charges (see Fig. 4.10 a).

For the QCD case, soft-gluon radiation in this direction is non-zero but appears to be suppressed owing to the same physical reason: replacing $\gamma$ by a qluon "recharges" $q$ and $\bar{q}$ to almost opposite color charges. Discussing "QCD Radiophysics" in Chapter 9 we shall derive the resulting ratio of multiplicity flows in symmetric $q \bar{q} \gamma$ and $q \bar{q} g$ events to be

$$
\frac{d N_{\gamma} / d \vec{n}}{d N_{g} / d \vec{n}}=\frac{2\left(N_{c}^{2}-1\right)}{N_{c}^{2}-2}=\frac{16}{7} .
$$

Note that the destructive interference is strong enough to make even the most kinematically unfavorable direction transverse to the event plane better populated as compared to the $q \bar{q}$ valley. In the case of the threefold symmetric (Mercedes-like) $q \bar{q} g$ events the ratio of particle flows reads

$$
\left(\frac{d N_{\perp} / d \vec{n}}{d N_{q \bar{q}} / d \vec{n}}\right)_{g}=\frac{N_{c}+2 C_{F}}{2\left(4 C_{F}-N_{c}\right)}=\frac{17}{14} .
$$

Let us notice that in the case of $q \bar{q} \gamma$ events this ratio is

$$
\left(\frac{d N_{\perp} / d \vec{n}}{d N_{q \bar{q}} / d \vec{n}}\right)_{\gamma}=\frac{1}{4} .
$$


Owing to the constructive interference there is a surplus of radiation in the $q g$ and $g \bar{q}$ valleys: for the Mercedes $q \bar{q} g$ configuration midway between the jet directions one gets

$$
\frac{d N_{q g}}{d N_{q \bar{q}}}=\frac{5 N_{c}^{2}-1}{2 N_{c}^{2}-4}=\frac{22}{7} .
$$

For hadron states associated with multijet events the ratios of particle flows given by (4.21)-(4.24) should remain correct, since non-PT hadronization effects should cancel, at least at high energies.

Studying the coherent phenomena one gets a plenty of infrared stable predictions deeply rooted in the basic structure of nonabelian gauge theory. The experimental observations of such phenomena can be said to test very detailed features of QCD color flows.

\subsection{Two-particle Correlations}

The energy-multiplicity correlations prove to be especially sensitive to colorflow structure in jet formation 1110. In the case of $e^{+} e^{-}$annihilation an interesting quantity is the gluon-gluon correlation function characterizing the emission of two soft gluons by a hard $q \bar{q}$ antenna 15 .

$$
C^{q \bar{q}}\left(\vec{n}_{3}, \vec{n}_{4}\right)=\frac{W^{q \bar{q}}\left(\vec{n}_{3}, \vec{n}_{4}\right)}{W^{q \bar{q}}\left(\vec{n}_{3}\right) W^{q \bar{q}}\left(\vec{n}_{4}\right)} .
$$

Here $W^{q \bar{q}}\left(\vec{n}_{i}\right)$ describes the radiation pattern for the $q \bar{q}$ antenna $(i=3,4)$ according to (4.13) and $W^{q \bar{q}}\left(\vec{n} 3, \vec{n}_{4}\right)$ represents the angular distribution of two soft gluons with $E_{4} \ll E_{3} \ll E_{2} \approx E_{1}$ with $E_{1}\left(E_{2}\right)$ the quark (antiquark) energy, and $\vec{n}_{1} \approx-\vec{n}_{2}$. 
To illustrate the specific features of the correlation function $C^{q \bar{q}}$ let us invoke once again the "QED" model used in the previous section, see Fig.4.11] Consider, for example, the configuration when the gluons are emitted at the same polar angles relative to the direction $\vec{n}_{1}\left(\Theta_{3}=\Theta_{4}\right)$ but in the back-to-back azimuthal directions $(\phi=\pi)$. Using the three-dimensional representation for the $q \bar{q}$ "g" radiation pattern given by (4.20) one can easily check that in this case $\vec{A}\left(\vec{n}_{3}\right) \| \vec{n} \equiv \vec{n}_{4}$ and the correlation function $C^{q \bar{q}}$ vanishes. This is a result of destructive interference, which is of the same magnitude as the drag effect in a symmetric $q \bar{q}$ "g" configuration.

In orthogonal azimuthal directions $(\phi=\pi / 2)$ the gluons become uncorrelated, $C^{q \bar{q}}=1$. Thus the gluon-gluon correlations in the orthogonal and backto-back azimuthal directions demonstrate the same interference phenomena as the drag effect.

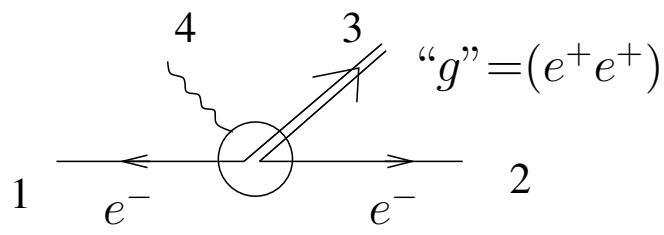

Figure 4.11: A "QED" model illustrating two-soft-gluon emission in $e^{+} e^{-} \rightarrow$ $q \bar{q}$.

The correlation function $C^{q \bar{q}}$ in the "QED" model can be presented in the form

$$
C^{q \bar{q}}\left(\eta_{34}, \phi\right)=1+2 \frac{\cos \phi}{\cosh \eta_{34}-\cos \phi}
$$

where $\eta_{34}=\eta_{3}-\eta_{4}$ with $\eta_{3}$ and $\eta_{4}$ gluon pseudorapidities, and $\phi=\phi_{3}-\phi_{4}$ 
relative azimuthal angle to the direction $\vec{n}_{1} \approx-\vec{n}_{2}$ :

$$
\cosh \eta_{34} \sin \Theta_{3} \sin \Theta_{4}=1-\cos \Theta_{3} \cos \Theta_{4}
$$

Equation (4.26) demonstrates explicitly the qualitative features of the correlation function discussed above. Corresponding correlation function for the real QCD case, very similar to (4.26), will be discussed in Section 4.6. In the solution to Problem 4.4 below one can find a description of simple and practically useful technique for deriving radiation patterns.

\subsection{Feynman-Gribov Puzzle and QCD Coherence}

Let us discuss some manifestations of coherent phenomena in DIS, see Ref. [8] for details. As it has been mentioned in Section 4.2. QCD coherence here, as in the case of a timelike cascade, leads to a picture with AO of soft gluon emissions.

When discussing the structure of the final state of a DIS process with momentum transfer $-Q^{2} \gg \Lambda^{2}$ and a fixed value of Bjorken variable $x$, one must take two phenomena into account: the dissociation of the initial parton fluctuation, the coherence of which was destroyed by the "removal" of a virtual quark (target fragmentation), and the evolution of the struck quark (current fragmentation).

As well known, these two fragmentation regions are best separated kinematically in the Breit frame $\left(Q_{0}=0,2 x \vec{p}=-\vec{Q}\right)$. Here the process looks like the abrupt spatial spreading of two color states: 3 (the struck quark $q$ ) and $\overline{3}$ (the disturbed proton), moving in the opposite directions. 

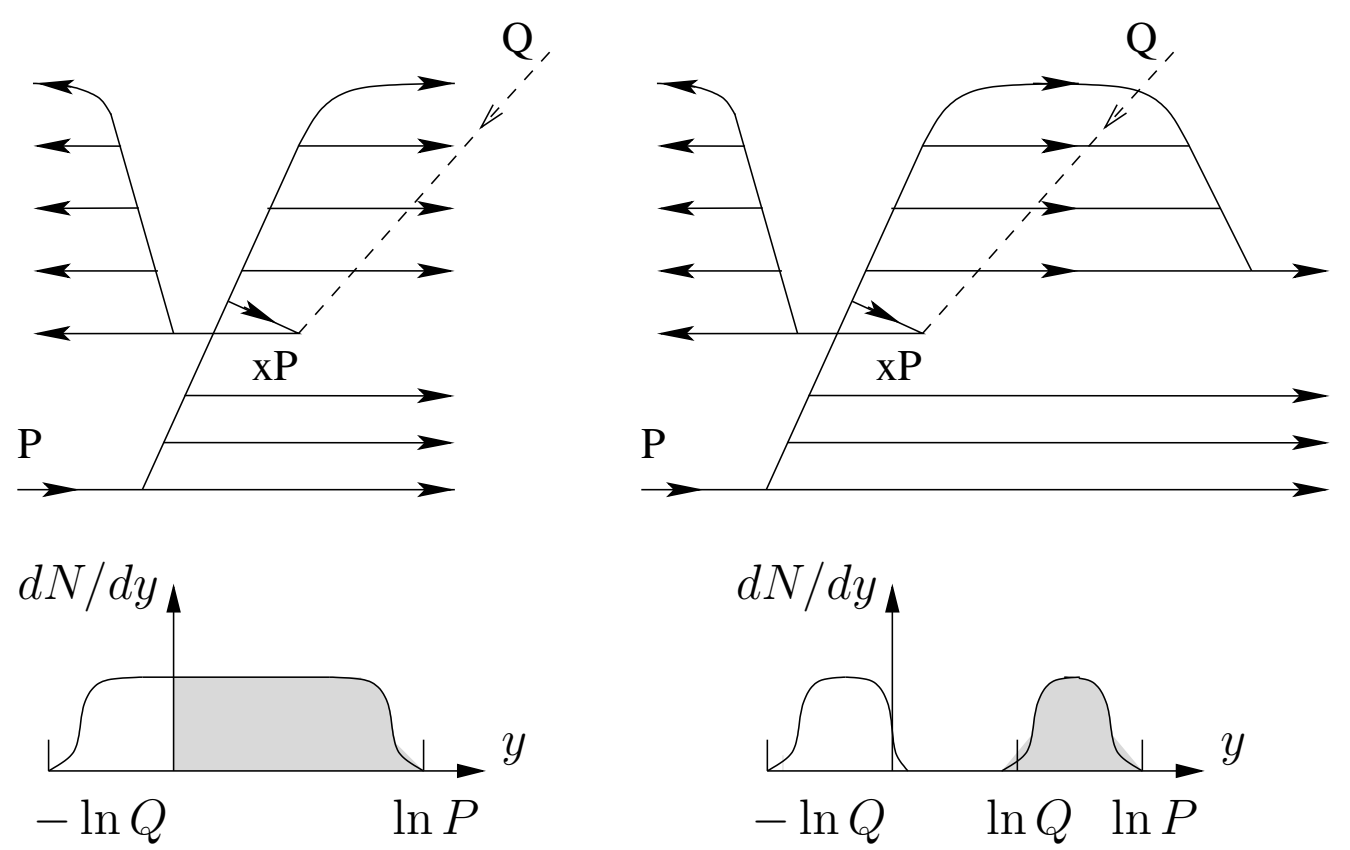

(a)

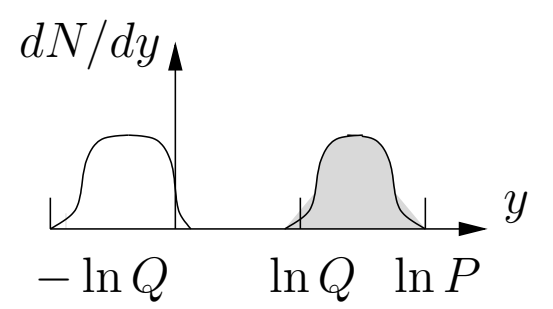

(b)

Figure 4.12: Structure of the hadron plateau in DIS process according to Feynman $(a)$ and Gribov $(b)$ (shaded area shows the target fragmentation region), $\quad y=\ln \omega$. 
Not the least role in the justification of the celebrated Feynman hypothesis about an universal hadronic plateau in DIS (see Fig. 4.12a) has been assigned to the argument that the continuously distributed hadrons connecting the two fragmentation regions are necessary to compensate the fractional charges of quarks-partons. At the same time, some serious doubts have been expressed about the possibility to organize dynamically such a state if one proceeds from the natural idea to consider successive decays of outgoing partons as an only source of multiple production of final particles. The problem has been formulated most clearly by Gribov in 1973 16. It was shown that in a DIS process the offsprings of the fragmentation of an initially prepared fluctuation had to leave the rapidity interval $0<\ln \omega<\ln Q$ depopulated as shown in Fig. 4.12b ( $\omega$ being the energy of registered hadron $h$ ).

At the basis of this conclusion an analysis lays of the space-time picture of the process development in the framework of a field-theoretical approach to the description of the wave function of the target hadron as a coherent system of partons. The absence of hadrons with momenta $\omega \ll Q$ in the target fragmentation in the Gribov picture can be simply explained by noticing that the coherence of the partonic wave function in this region remains, in fact, not destroyed by hard knocking out a parton with momentum $x P \approx Q / 2$, as a consequence of which the upper (low momenta) part of the partonic fluctuation in Fig. 4.12b "collapses" as if there were no scattering at all.

The experimental observation of an uniform flat plateau (Fig. 4.12 $\mathrm{a}$ ), which was interpreted as a proof of the correctness of the identification of partons with quarks, did not eliminate the Feynman-Gribov puzzle 3 but only sharpened it in fact, since it required one to point out a clear physical mechanism responsible for the filling of the "Gribov's gap", that would not come into conflict with quantum mechanics.

\footnotetext{
${ }^{3}$ we are not sure that Feynman himself ever heard of such a term
} 
From the present-day standpoints the foundations for the Gribov phenomenon have only been strengthened, since QCD indeed corroborated the treatment of the structure of a relativistic hadron in terms of field fluctuations composed of quasi-real quarks and gluons - the partons of QCD.

The way in which this puzzle is resolved in the QCD context 8 appears to be eclectic in a certain sense. On the one hand, here the Gribov phenomenon does occur indeed: the energy spectrum of the offsprings of the parton dissociation - elements of the "ladder" fluctuations determining the DIS cross-section - proves to be concentrated mainly in the region of large energies $Q<\omega<P$. On the other hand, there is a specific mechanism responsible for bridging the regions of target and current fragmentation in the spirit of the Feynman picture. This role is taken on by coherent bremsstrahlung of soft gluons which is insensitive to details of the structure of partonic wave function of the target hadron, being governed exclusively by the value of the total color charge transferred in the scattering process (see Fig. 4.45).

The fact that at small $x$ 's where the "sea" contribution dominates, the scattering amplitude corresponding to the gluon color transfer in the $t$ channel makes the accompanying radiation in the target fragmentation region approximately twice as intensive as that in the fragmentation of current. 
Problem 4.1 Using (4.10) find the angular distribution of particles for coherent and incoherent cases.

\section{Answer:}

$$
\begin{aligned}
\rho^{c o h}(\Theta) & =1+\frac{\alpha_{s}}{2} \ln ^{2}(E \Theta R) \\
\rho^{\text {incoh }} & =1+\alpha_{s} \ln ^{2}(E \Theta R)
\end{aligned}
$$

Notice, that here there is no qualitative difference between two approaches contrary to the energy spectra (4.11).

Problem 4.2 Derive the classical representation of radiation pattern (4.20).

Problem 4.3 Using the expression (4.12) for $q \bar{q}$ antenna pattern derive the relation (4.23).

Problem 4.4 Derive the expression (4.26) for the correlation function $C^{q \bar{q}}$ in the "QED" model.

\section{Solution:}

Rewrite $W^{q \bar{q} "} g "\left(\vec{n}_{4}\right)$ from (4.20) in a standard form

$$
W^{q \bar{q} "} g^{\prime \prime}=\sum_{i=1,2}\left(\vec{A} \cdot \vec{e}_{i}\right)^{2}
$$

with $\vec{e}_{i}$ the physical polarizations of a gluon $g_{4}: \vec{e}_{i}^{2}=1,\left(\vec{e}_{i} \cdot \vec{n}_{4}\right)=0$.

We can choose vector $\vec{e}_{1}$ laying in the plane formed by vectors $\vec{n}_{1}, \vec{n}_{4}$ and $\vec{e}_{2}$ being orthogonal to this plane. According to 4.20b) $\vec{A} \cdot \vec{e}_{2}=0$ at $\phi \equiv \phi_{3}-\phi_{4}=0, \pi$. 
Using (4.29) one can present $C^{q \bar{q}}\left(\eta_{34}, \phi\right)$ as a sum of two contributions $C_{i}^{q \bar{q}}$ corresponding to the polarizations $\vec{e}_{i}$ :

$$
\begin{aligned}
C^{q \bar{q}}(\eta, \phi) & =C_{1}^{q \bar{q}}(\eta, \phi)+C_{2}^{q \bar{q}}(\eta, \phi) \\
C_{1}^{q \bar{q}} & =\frac{\cosh ^{2} \eta-1}{(\cosh \eta-\cos \phi)^{2}} \\
C_{2}^{q \bar{q}} & =\frac{\sin ^{2} \phi}{(\cosh \eta-\cos \phi)^{2}} .
\end{aligned}
$$

$C_{1}$ vanishes when $\Theta_{3}=\Theta_{4}\left(\eta_{34}=0\right)$, as $C_{2}$ does at $\phi=\pi$. At $\phi=\pi / 2$ one gets $C_{1}^{q \bar{q}}+C_{2}^{q \bar{q}}=1$ and there are no correlations. 


\section{Bibliography}

1. Yu.L. Dokshitzer, V.A. Khoze, A.H. Mueller and S.I. Troyan. Rev. Mod. Phys., 60:373, 1988.

2. Yu.L. Dokshitzer, V.A. Khoze and S.I. Troyan. Coherence and physics of QCD jets. In A.H. Mueller, editor, Perturbative QCD, page 241. World Scientific, Singapore, 1989.

3. A.E. Chudakov. Izv. Akad. Nauk SSSR, Ser. Fiz., 19:650, 1955.

4. Yu.L. Dokshitzer and S.I. Troyan. Proceedings of the XIX Winter School of the LNPI, vol. 1, page 144. Leningrad, 1984.

Yu.L. Dokshitzer and S.I. Troyan. Preprint LNPI-922, 1984.

5. G. Marchesini and B.R. Webber. Nucl. Phys., B238:1, 1984.

B.R. Webber. Nucl. Phys., B238:492, 1984.

6. B. Bambach et al. QCD Generators at LEP. In G. Altarelli, R. Kleiss, and C. Verzegnassi, editors, Proceedings of the Workshop on $Z$ physics at LEP, CERN Report 89-08, vol. 3, p. 43, 1989.

T. Sjöstrand. CERN TH. 5902 / 1990.

7. M. Ciafaloni. Nucl. Phys., B296:249, 1987.

8. L.V. Gribov, Yu.L. Dokshitzer, S.I. Troyan and V.A. Khoze. Sov. Phys. JETP, 68:1303, 1988.

9. S. Catani, F. Fiorani and G. Marchesini. Nucl. Phys., B336:18, 1990.

10. Yu.L. Dokshitzer, V.A. Khoze and S.I. Troyan. In M. Derrick, editor, Proceedings of the 6th Int. Conf. on Physics in Collisions, page 417. World Scientific, Singapore, 1987.

11. Ya.I. Azimov, Yu.L. Dokshitzer, V.A. Khoze and S.I. Troyan. Z. Phys., C31:213, 1986. 
12. Ya.I. Azimov, Yu.L. Dokshitzer, V.A. Khoze and S.I. Troyan. Phys. Lett., 165B:147, 1985. Yad. Phys., 43:149, 1986.

13. B.R. Webber. In Proceedings of the XVI Int. Symposium on Multiparticle Dynamics, Kiryat Anavim, page 41. 1985.

14. L.D. Landau and E.M. Lifshitz. The classical theory of fields. AddisonWesley Physics Series, 1951.

15. Yu.L. Dokshitzer, V.A. Khoze, G. Marchesini and B.R. Webber. Phys. Lett., 245:243, 1990.

16. V.N. Gribov. Proceedings of the VIII Winter School of the LNPI, volume 2, page 5. Leningrad, 1973. 


\section{Chapter 5}

\section{Double Logarithmic Approximation}

\section{Contents}

5.1 Tree Multigluon Amplitudes for $e^{+} e^{-} \rightarrow q \bar{q}+N d$. 145

5.1 .1 Two Gluon Emission off a Quark . . . . . . . . . . 147

5.1 .2 Angular Ordering for $N=2$. . . . . . . . . 150

5.2 Angular Ordering in All Orders . . . . . . . . 152

5.3 Virtual Corrections . . . . . . . . . . 157

5.4 Method of Generating Functionals . . . . . . . 158

5.4 .1 Exclusive and Inclusive Cross Sections . . . . . . . 159

5.4.2 Generating Functional (GF)s for $q$ and $q$ Jets . . . 160

5.5 Multiplicity Distributions in QCD Jets . . . . 165

5.6 KNO Phenomenon . . . . . . . . . . 170 


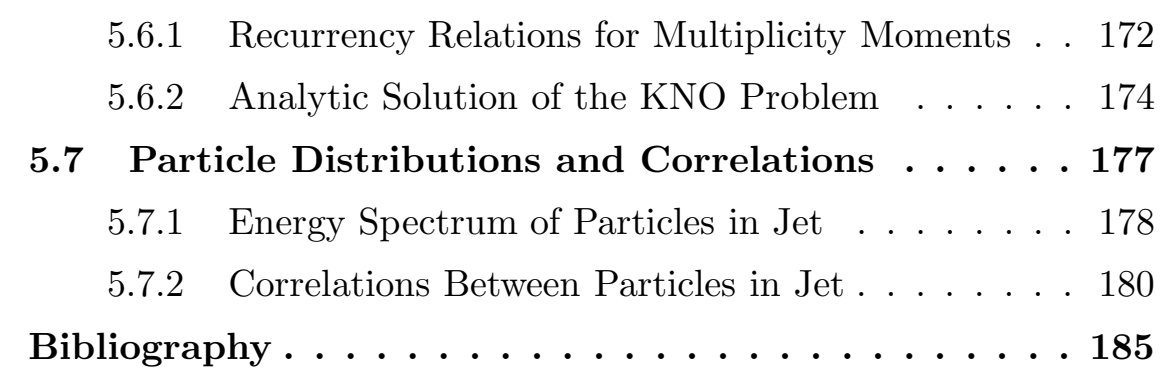

With this Chapter we begin to study properties of soft particle distributions in jets. Following the historical line of development of PT technique as well as for pedagogical reasons we start with an analysis of pure gluonic systems in a rough approximation which accounts for only leading double logarithmic contributions to multiparton cross sections (DLA)

$$
\frac{\alpha_{s}}{\pi} \ll 1 \quad, \quad \frac{\alpha_{s}}{\pi} \log Q^{2} \ll 1 \quad, \quad \frac{\alpha_{s}}{\pi} \log ^{2} Q^{2} \sim 1 .
$$

DLA analysis will exhibit the main physical ingredients of the problem, namely, the structure of intrajet parton cascades and the role of QCD coherence effects in soft gluon multiplication processes. After constructing the multigluon amplitudes $M_{N}$ corresponding to the most probable bremsstrahlung patterns, we shall see how the strong angular ordering (AO) helps to present the cross section $\left|M_{N}\right|^{2}$ in terms of a classical shower picture with the successive $g \rightarrow g g$ emissions forming the Markov chains of independent elementary radiation events, observe an interesting property of multiplicity fluctuations in QCD (KNO scaling) and make a list of DLA predictions for parton spectra and correlations which may serve as a useful qualitative guide to jet physics. The first formal part of the DLA analysis splits into three 
problems: construction of tree $\mathrm{N}$-gluon amplitudes, proof of $\mathrm{AO}$ and account of virtual (loop) corrections.

\subsection{Tree Multigluon Amplitudes for $e^{+} e^{-} \rightarrow$ $q \bar{q}+N g$}

As a field for studying jet evolution it is natural to choose the simplest QCD process, namely, $e^{+} e^{-} \rightarrow q \bar{q}$.

Notation has to be fixed first. Hereafter we shall denote by

\begin{tabular}{||ccc||}
\hline$p_{+}=\left(E_{+}, \vec{p}_{+}\right)$ & - & 4-momentum of the quark $q$, \\
$p_{-}=\left(E_{-}, \vec{p}_{-}\right)$ & - & 4-momentum of the antiquark $\bar{q}$, \\
$k_{i}=\left(\omega_{i}, \vec{k}_{i}\right)$ & - & 4-momentum of the $i$-th soft gluon $g_{i}$, \\
$e_{i \mu}^{(\lambda)}$ & - & its polarization vector $(\lambda=0, \ldots, 3)$, \\
$a_{i}$ & - & the color index of the gluon $i$.
\end{tabular}

Strong Energy Ordering. In the DLA we are looking for the $N$-gluon production cross section as large as

$$
\sigma_{N} \propto \int\left|M_{N}\right|^{2} \prod_{i=1}^{N} \frac{d^{3} k_{i}}{2 \omega_{i}} \propto\left(g_{s}^{2} \log ^{2}\right)^{N} .
$$

In order to gain such a contribution each gluon must contribute two big logarithms: energy and collinear ones. This means that we have to consider 
gluons strongly ordered in their energies

$$
E_{+(-)} \gg \omega_{1} \gg \omega_{2} \gg \ldots \gg \omega_{N}
$$

since the kinematical region where any two of them have energies of the same order of magnitude, $\omega_{i} \sim \omega_{k}$, would result in a loss of at least one energy log.

Gauge Choice. To simplify the analysis of the angular logs it is useful to choose a specific ( "physical") gauge, where the gluon emission vertex, either $q \rightarrow q g$ or $q \rightarrow g g$ vanishes at collinear momenta. Making use of the LLA experience (see Chapter 11) we choose the planar gauge where the gluon propagator reads

$$
\begin{aligned}
G_{\mu \nu}^{a b}(k) & =\delta^{a b} \frac{d_{\mu \nu}(k)}{k^{2}+i \epsilon} \\
d_{\mu \nu}(k) & =g_{\mu \nu}-\frac{k_{\mu} c_{\nu}+c_{\mu} k_{\nu}}{(k c)} .
\end{aligned}
$$

It is convenient to take the gauge vector $c_{\mu}$ proportional to the total $4-$ momentum of the $e^{+} e^{-}$pair:

$$
c_{\mu}=(1, \vec{c}) \quad ; \quad \vec{c}=0 \quad \text { in the } e^{+} e^{-} \mathrm{cms} .
$$

In this gauge $q$ and $\bar{q}$ emit soft gluons independently since the interference between two emission amplitudes vanishes due to the property

$$
p_{+}^{\mu} d_{\mu \nu}(k) p_{-}^{\nu}=0 .
$$

In calculating the cross section (5.2) one has to sum the amplitudes squared for production of two "physical" polarizations $e_{\mu}^{(1)}$ and $e_{\mu}^{(2)}$ of $N$ "real" gluons:

$$
\left(e_{i}^{(1,2)}\left(k_{i}\right) \cdot k_{i}\right)=0 \quad, \quad\left(e_{i}^{(1,2)} \cdot c\right)=0 \quad, \quad(e)^{2}=-1 .
$$


Analyzing Feynman diagrams we'll be faced also with a plenty of virtual lines propagating all the four possible polarizations of vector gluon fields. However, an advantage of the gauge (5.4) (and this explains the reason to call it physical) is that it diminishes the contributions coming from the two nonphysical polarizations $e_{\mu}^{(0)}, e_{\mu}^{(3)}$ in the virtual gluon propagators:

$$
\begin{aligned}
d_{\mu \nu}(k) & =-\sum_{\lambda=0}^{3} e_{\mu}^{(\lambda)}(k) e_{\nu}^{(\lambda)}(k), \\
e_{\mu}^{(0,3)}(k) & =\frac{k_{\mu} \pm \sqrt{k^{2}} c_{\mu}}{\left[2 \omega\left(\omega \pm \sqrt{k^{2}}\right)\right]^{1 / 2}}
\end{aligned}
$$

where $\omega=(k c)$ is the gluon energy.

\subsubsection{Two Gluon Emission off a Quark}

Feynman amplitudes corresponding to the graphs of Fig. 5.1 can be written as

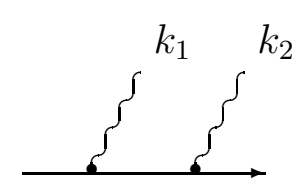

a

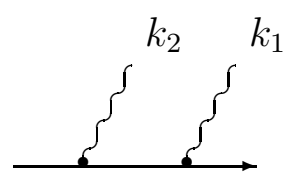

(b)
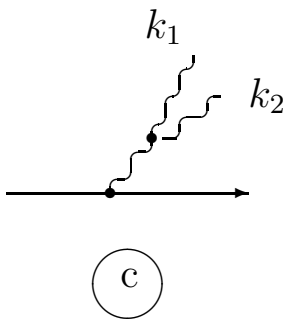

Figure 5.1: Feynman amplitudes for $e^{+} e^{-} \rightarrow q \bar{q} g_{1} g_{2}$. 


$$
\begin{aligned}
& M_{a}=g_{s}^{2} \frac{e_{2} p_{+}}{k_{2} p_{+}} \frac{e_{1} p_{+}}{\left(k_{1}+k_{2}\right) p_{+}} * t^{a_{2}} t^{a_{1}}, \\
& M_{b}=g_{s}^{2} \frac{e_{1} p_{+}}{k_{1} p_{+}} \frac{e_{2} p_{+}}{\left(k_{2}+k_{1}\right) p_{+}} * t^{a_{1}} t^{a_{2}}, \\
& M_{c}=g_{s}^{2} e_{1}^{\mu} e_{2}^{\nu} \gamma_{\mu \nu \rho}\left(k_{1}, k_{2},-k\right) \frac{d^{\rho \sigma}(k)}{k^{2}} \frac{p_{+\sigma}}{k p_{+}} * i f_{a_{1} a_{2} c} t^{c}
\end{aligned}
$$

where $k=k_{1}+k_{2}$ stands for the virtual gluon momentum and

$$
\gamma_{\mu \nu \rho}\left(k_{1}, k_{2}, k_{3}\right)=g_{\mu \nu}\left(k_{2}-k_{1}\right)_{\rho}+g_{\rho \mu}\left(k_{1}-k_{3}\right)_{\nu}+g_{\nu \rho}\left(k_{3}-k_{2}\right)_{\mu}
$$

is the three-gluon QCD vertex. Singular behavior with respect to the directions of gluon momenta $\vec{n}_{i}$ may come only from Feynman denominators (notice, that $d^{\rho \sigma}(k)$ contains no angular dependence in its denominator, see (5.4) ). Therefore the kinematical regions where both $\vec{n}_{1}$ and $\vec{n}_{2}$ integrations are logarithmic can be shown to be the following:

$$
\begin{aligned}
\Theta_{1}^{2} & \gg \frac{\omega_{2}}{\omega_{1}} \Theta_{2}^{2}, \\
\Theta_{2}^{2} & \gg \frac{\omega_{1}}{\omega_{2}} \Theta_{1}^{2}, \\
\frac{\omega_{1}}{\omega_{2}} \Theta_{1}^{2} & \gg \Theta_{2}^{2} \gtrsim \Theta_{1}^{2}
\end{aligned}
$$

for the amplitudes of Fig. 5.1 - - c respectively. Diagrams (a) and (b) are of QED type, so let us concentrate on the third amplitude Fig. 5.1p :

$$
2\left(k p_{+}\right) \approx E_{+}\left(\omega_{1} \Theta_{1}^{2}+\omega_{2} \Theta_{2}^{2}\right) \quad, \quad k^{2} \approx \omega_{1} \omega_{2} \Theta_{12}^{2} .
$$

In the above $\Theta_{1}\left(\Theta_{2}\right)$ is the angle between $\vec{p}_{+}$and $\vec{k}_{1}\left(\vec{k}_{2}\right) ; \quad \Theta_{12}$ is the angle between $\vec{k}_{1}$ and $\vec{k}_{2}$. Making use of (15.7c) one has as an estimate of the different 
polarization states of the virtual gluon $k$ :

$$
\begin{aligned}
& e_{1}^{\mu} e_{2}^{\nu} \gamma_{\mu \nu \rho}\left(k_{1}, k_{2},-k\right) e_{\rho}^{(1,2)}(k) \sim \omega_{1} \Theta_{12} \gg \sqrt{\omega_{1} \omega_{2} \Theta_{12}^{2}} \approx \sqrt{k^{2}} \sim e_{1}^{\mu} e_{2}^{\nu} \gamma_{\mu \nu \rho} e_{\rho}^{(0,3)}(k), \\
& e_{\sigma}^{(1,2)}(k) p_{+}^{\sigma} \sim \frac{E_{+}}{\omega_{1}}\left(\omega_{1} \Theta_{1}+\omega_{2} \Theta_{2}\right) \gg \\
& \quad \frac{E_{+}}{\omega_{1}}\left(\omega_{1} \Theta_{1}^{2}+\omega_{2} \Theta_{2}^{2} \pm \sqrt{\omega_{1} \omega_{2} \Theta_{12}^{2}}\right) \sim \frac{\left(p_{+} k\right) \pm \sqrt{k^{2}}\left(p_{+} c\right)}{\omega} \sim e_{\sigma}^{(0,3)} p_{+}^{\sigma} .
\end{aligned}
$$

These inequalities apparently show that nonphysical polarizations $e^{(0,3)}(k)$ prove to be negligible indeed. Therefore, the $d_{\rho \sigma}(k)$ factor in (5.5c) can be approximated by the transverse tensor

$$
\begin{aligned}
g_{\rho \sigma}^{\perp}(k) & \equiv-\sum_{\lambda=1,2} e_{\rho}^{(\lambda)}(k) e_{\sigma}^{(\lambda)}(k), \\
g_{\rho 0} & =g_{0 \sigma}=g_{00}=0, \\
g_{i j} & =\delta_{i j}-\frac{k_{i} k_{j}}{\vec{k}^{2}} \quad(i, j=1 \ldots 3),
\end{aligned}
$$

and the gluon vertex (5.6) by the dominant term

$$
\begin{aligned}
\gamma_{\mu \nu \sigma}\left(k_{1}, k_{2},-k\right) & \approx g_{\mu \rho}\left(2 k_{1}+k_{2}\right)_{\nu}, \\
e_{1}^{\mu} e_{2}^{\nu} \gamma_{\mu \nu \rho}\left(k_{1}, k_{2},-k\right) d_{\rho \sigma} p_{+}^{\sigma} & \approx 2\left(e_{2} k_{1}\right) e_{1}^{\mu} g_{\mu \sigma}^{\perp}(k) p_{+}^{\sigma} .
\end{aligned}
$$

Finally, using (5.7c) to estimate

$$
\vec{e}_{1} \vec{p}_{+} \sim E_{+} \Theta_{1} \gg \frac{\left(\vec{e}_{1} \vec{k}\right)\left(\vec{k} \vec{p}_{+}\right)}{\vec{k}^{2}} \sim E_{+} \frac{\omega_{2}}{\omega_{1}} \Theta_{12}
$$


one verifies the possibility to replace $g_{\mu \sigma}^{\perp}$ in (5.11) by the unit tensor $g_{\mu \sigma}$ :

$$
e_{1}^{\mu} g_{\mu \sigma}^{\perp}(k) p_{+}^{\sigma}=-\left(\vec{e}_{1} \vec{p}_{+}\right)+\frac{\left(\vec{e}_{1} \vec{k}\right)\left(\vec{k} \vec{p}_{+}\right)}{\vec{k}^{2}} \approx-\left(\vec{e}_{1} \vec{p}_{+}\right)=e_{1}^{\mu} g_{\mu \sigma} p_{+}^{\sigma} .
$$

The resulting DL expression for the gluonic amplitude (5.7C) of Fig. 5.1p looks very much alike (5.5a) and (5.5b):

$$
M_{c}=g_{s}^{2} \frac{e_{2} k_{1}}{k_{2} k_{1}} \frac{e_{1} p_{+}}{\left(k_{1}+k_{2}\right) p_{+}} * i f_{a_{1} a_{2} c} t^{c} .
$$

\subsubsection{Angular Ordering for $N=2$}

A further simplification of the DL amplitudes (5.5a), (5.5b) and (5.12) is connected with the formulation of a shower picture. From the kinematical restrictions (5.7) one concludes that the DL regions $(a)$ and (c) overlap, and, thus, the corresponding amplitudes interfere. It proves to be possible, however, to avoid an examination of the interference contribution. To see this let us consider three non-overlapping angular regions, namely

$$
\begin{aligned}
\text { I. } & \Theta_{1} \gg \Theta_{2} \\
\text { II. } & \Theta_{2} \gg \Theta_{1} \\
\text { III. } & \Theta_{12} \ll \Theta_{1} \approx \Theta_{2}
\end{aligned}
$$

and show that the matrix element in each of these regions takes the form of a product of trivial independent radiation factors.

In the region $I$ the only contribution comes from the amplitude of Fig. 5.1 a which takes the form $\left(k_{1} p_{+} \gg k_{2} p_{+}\right)$

$$
M_{I}=g_{s}^{2} \frac{e_{2} p_{+}}{k_{2} p_{+}} \frac{e_{1} p_{+}}{k_{1} p_{+}} * t^{a_{2}} t^{a_{1}}
$$


The kinematical inequality II splits into two subregions:

$$
\Theta_{2}^{2} \gg \frac{\omega_{1}}{\omega_{2}} \Theta_{1}^{2}
$$

and

$$
\frac{\omega_{1}}{\omega_{2}} \Theta_{1}^{2} \gg \Theta_{2}^{2} \gg \Theta_{1}^{2} .
$$

In the first case $(c f .(5.7 \mathrm{~b}))$ only the graph of Fig. $5.1 \mathrm{~b}$ contributes as $\left(k_{2} p_{+} \gg\right.$ $\left.k_{1} p_{+}\right)$

$$
M_{I I}=g_{s}^{2} \frac{e_{1} p_{+}}{k_{1} p_{+}} \frac{e_{2} p_{+}}{k_{2} p_{+}} * t^{a_{1}} t^{a_{2}} .
$$

In the second subregion one has to account for both Figs. 5.17 and 5.17. Here, however,

$$
\Theta_{12} \approx \Theta_{2} \quad, \quad \frac{e_{2} k_{1}}{k_{2} k_{1}} \approx \frac{e_{2} p_{+}}{k_{2} p_{+}}
$$

and summing (5.5a) and (5.12), with account of the commutation relation

$$
\left[t^{a_{2}} t^{a_{1}}\right]=i f_{a_{1} a_{2} c} t^{c}
$$

and the inequality (5.7a), one easily arrives at (5.15). Thus, the matrix element (5.15) proves to be correct all over the kinematical region $I I$ of (5.13).

Finally, the diagram of Fig. 5.1 dominates in the region III of a quasicollinear $g_{1} g_{2}$ pair. Here $k_{1} p_{+} \gg k_{2} p_{+}$and the matrix element reads

$$
M_{I I I}=g_{s}^{2} \frac{e_{2} k_{1}}{k_{2} k_{1}} \frac{e_{1} p_{+}}{k_{1} p_{+}} * i f_{a_{1} a_{2} c} t^{c} .
$$

To make the future generalization transparent let us formulate the answer in terms of a classical chain of sequential branching processes. 
- Fig. $5.1 \mathrm{a}(\mathrm{b})$ : the quark with momentum $p_{+}$emits first the gluon $k_{1}\left(k_{2}\right)$ and then $k_{2}\left(k_{1}\right)$; we attribute the angular region $I(I I)$ to this graph.

- Fig. 5.1k: the quark emits the gluon $k_{1}$ which, in turn, emits the softer gluon $k_{2}$; the region $I I I$.

- Elementary radiation contributes to the matrix element by the classical bremsstrahlung factor.

- Emission angles strongly decrease along each chain.

- The color factor corresponds directly to the classical graph describing the genealogy of the given process.

\subsection{Angular Ordering in All Orders}

Construction Rules for getting the whole set of $N$-gluon amplitudes in DLA. An interested reader, who has walked through the previous subsection with a pencil and paper, is ready now to formulate these with us. Here they are.

Draw a tree Feynman diagram $\mathcal{D}$ without 4 -gluon vertices.

Group gluon propagators into $N$ "gluon lines" — sets of virtual $g$ states with approximately the same energies (this is possible due to strong energy ordering (5.3) which are depicted by straight lines in Fig. 5.2

Call $i$ the parent and $j$ the offspring for each vertex $i \rightarrow i+j \quad(i>j)$.

Define the region $\Gamma_{\mathcal{D}}$ in the space of emission angles, corresponding to $\mathcal{D}$ : angles decrease along each path in the tree, starting from the $\gamma^{*} \rightarrow q \bar{q}$ vertex. 
Build the color factor $G$ for $\mathcal{D}$ according to the usual Feynman rules:

$\rightarrow t^{a} \equiv \lambda^{a} / 2$ for any $q(\bar{q}) \rightarrow q(\bar{q})+g$ vertex,

$\rightarrow i f_{a b c}$ for a $3-g$ vertex, where $a(b)$ mark the gluon with the lowest (highest) energy.

Attribute to the diagram $\mathcal{D}$ in the angular region $\Gamma_{\mathcal{D}}$ the matrix element

$$
M_{N}=g_{s}^{N}(-1)^{m} \prod_{i=1}^{N} \frac{\left(e_{i} \mathcal{P}_{i}\right)}{\left(k_{i} \mathcal{P}_{i}\right)} * G,
$$

where $m$ is the number of gluons emitted by $\bar{q}, \quad \mathcal{P}_{i}$ the 4 -momentum of the parent of the gluon $i$.

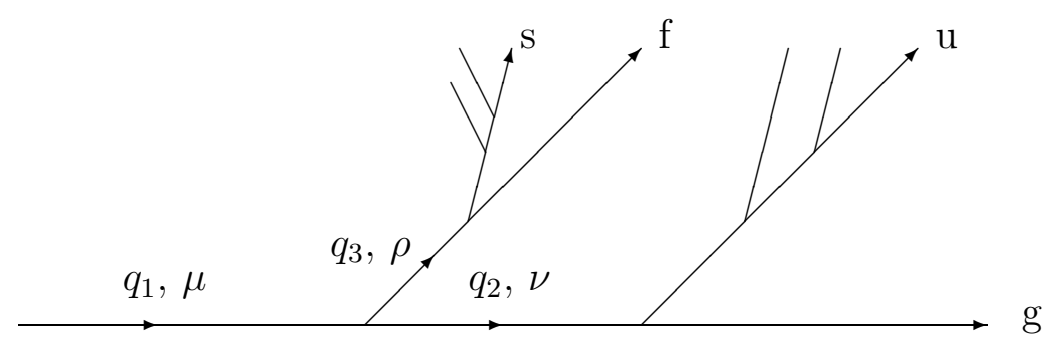

Figure 5.2: Scheme of a gluon cascade.

Noteworthy to mention, $\mathcal{P}_{i}$ is the momentum of one of the final ("real") partons and not that of any virtual state. Equation (5.17) represents the so-called "soft insertion" rules (for review see, e.g., Ref. (1). 
Formal Proof of AO in DLA follows, in fact, the logic elaborated for the $N=2$ case. The detailed proof has been given by Fadin in Ref. 20 . Let us enumerate and discuss briefly the main steps of the proof and leave the details to the reader.

1. Simplify the denominators of virtual propagators:

$$
q_{i}^{2}=\left(\sum_{t} k_{t}\right)^{2} \approx 2 k_{i} \sum_{t \neq i} k_{t}
$$

where the sum runs over all the final products $k_{t}$ of a given virtual particle $i, k_{i}$ being the most energetic among them. Equation (5.18) follows from the chain of estimates:

$$
k_{i}\left(k_{j}+k_{l}\right) \sim \omega_{i}\left(\omega_{j} \Theta_{i j}^{2}+\omega_{l} \Theta_{i l}^{2}\right) \gg \omega_{j} \omega_{l}\left(\Theta_{i j}^{2}+\Theta_{i l}^{2}\right) \gtrsim \omega_{j} \omega_{l} \Theta_{j l}^{2} \sim k_{j} k_{l}
$$

( remind that $\omega_{i} \gg \omega_{j}, \omega_{l}$ ).

2. For each vertex $V$ in the tree (see Fig. 5.2) impose the angular restrictions, necessary to pick up all the angular logs:

$$
\begin{array}{ccc}
\omega_{f} \Theta_{f g}^{2} & \gg \omega_{s} \Theta_{s f}^{2}, \\
\omega_{f} \Theta_{f g}^{2} & \gg \omega_{u} \Theta_{u g}^{2},
\end{array}
$$

where "s" marks the eldest son of " $f$ ", " $u$ " — its uncle, the eldest among younger brothers of " $f$ " (see Fig. 5.2).

3. Show that due to (5.19) virtual propagators of "elder generations", e.g., all those between $V$ and the $q \bar{q}$ creation point prove to be independent of $k_{s}$ and $k_{u}$. 
4. Prove that the DLA contribution may come only from the angular region where the inequalities (5.19) are fulfilled for each vertex, showing that the violation of this restriction somewhere in the tree would lead to a loss of at least one $\Theta$-logarithm.

5. Simplify the numerators using the planar gauge and physical polarizations for the final gluons. Here one has to prove the dominance of two physical polarizations $\lambda_{i}=1,2$ for virtual gluon propagators surrounding each vertex $V$ :

$$
\begin{aligned}
V & =e_{\mu}^{\lambda_{1}}\left(q_{1}\right) e_{\nu}^{\lambda_{2}}\left(q_{2}\right) e_{\rho}^{\lambda_{3}}\left(q_{3}\right) \gamma^{\mu \nu \rho}\left(-q_{1}, q_{2}, q_{3}\right) \\
& \approx 2\left(e_{\mu}^{\lambda_{3}}\left(q_{3}\right) k_{g}^{\mu}\right)\left(e^{\lambda_{1}}\left(q_{1}\right) e^{\lambda_{2}}\left(q_{2}\right)\right),
\end{aligned}
$$

where $q_{10} \approx q_{20} \approx \omega_{g} ; \quad q_{30} \approx \omega_{f}$.

6. Consider then the chain of vertices along the line $f$ and check, similarly to the $N=2$ case, the validity of the substitutions

$$
d_{\mu \nu}\left(q_{i}\right) \Longrightarrow-\sum_{\lambda=1,2} e_{\mu}^{\lambda}\left(q_{i}\right) e_{\nu}^{\lambda}\left(q_{i}\right)=g_{\mu \nu}^{\perp} \Longrightarrow g_{\mu \nu}
$$

and you will arrive finally at the desired (5.17).

7. The last step is to check the possibility to replace the DL angular regions (5.19), which may coincide partially for different diagrams, by the nonoverlapping $\mathrm{AO}$ regions $\Gamma_{\mathcal{D}}$. This can be proved by induction.

Cross Section. To obtain $N$-gluon production cross sections we havo to sum up the amplitudes squared of (5.17) for all the graphs $\mathcal{D}$. Performing a 
sum over the physical polarizations $\lambda=1,2$ of each soft gluon produced, one gets with the help of (5.8)

$$
d \sigma_{N}=d \sigma_{0} \prod_{i=1}^{N} \frac{g_{s}^{2}}{(2 \pi)^{3}} C \frac{d^{3} k_{i}}{2 \omega_{i}} \frac{\overrightarrow{\mathcal{P}}_{i}^{2} \sin ^{2} \Theta_{k_{i}}}{\left(k_{i} \mathcal{P}_{i}\right)^{2}},
$$

where $d \sigma_{0}$ denotes the Born annihilation cross section and $C$ is the color charge of the parent of a given gluon $i: C_{F} \equiv\left(N_{c}^{2}-1\right) / 2 N_{c}=4 / 3$ for emission off a quark or $C_{V} \equiv N_{c}=3$ for $g \rightarrow g g$ cascades.

Since we are interested in the region of parametrically small angles, the radiation factors can be simplified as

$$
\frac{\overrightarrow{\mathcal{P}}_{i}^{2} \sin ^{2} \Theta_{k_{i}}}{\left(k_{i} \mathcal{P}_{i}\right)^{2}}=\frac{1}{\omega^{2}} \frac{\sin ^{2} \Theta_{k_{i}}}{\left(1-\cos \Theta_{k_{i}}\right)^{2}} \approx \frac{4}{\omega^{2} \Theta_{k_{i}}^{2}} \approx \frac{4}{k_{i \perp}^{2}}
$$

so that after implementing $\alpha_{s} \equiv g_{s}^{2} / 4 \pi$ we rewrite the differential cross sections of single gluon emission from (5.20) in the form which shows most transparently the DL-character of gluon radiation:

$$
d \mathcal{K}(\vec{k}) \equiv \frac{d \omega}{\omega} \frac{d^{2} k_{\perp}}{2 \pi k_{\perp}^{2}} \frac{2 C \alpha_{s}}{\pi} .
$$

With account of the DLA virtual corrections to the tree amplitude $\mathcal{D}$ (see below), the cross section takes finally the form

$$
d \sigma=d \sigma_{0} \sum_{\mathcal{D}} \mathcal{F}^{2} \prod_{i} d \mathcal{K}\left(\vec{k}_{i}\right) .
$$

It is important to remember that for each tree graph $\mathcal{D}$ in (5.22) the produced gluons $k_{1} \ldots k_{N}$ are ordered with respect to their emission angles according to $\Gamma_{\mathcal{D}}$ condition:

$$
\Gamma(\mathcal{P}, \Theta):\left\{k^{0} \equiv \omega<\mathcal{P}^{0} ; \quad \Theta_{\widehat{\vec{k}} \overrightarrow{\mathcal{P}}} \equiv \Theta_{k}<\Theta ; \quad k_{\perp} \approx \omega \Theta_{k}>Q_{0}\right\},
$$


where $\mathcal{P}$ is the momentum of the parent of a given parton $k, \Theta$ the angle of the previous parton splitting on the $\mathcal{P}$-line.

\subsection{Virtual Corrections}

Taking into account virtual corrections results in the multiplication of the matrix element (5.17) by the factor

$$
\mathcal{F}=\exp -\frac{1}{2}\left[w_{F}\left(p_{+}, 1\right)+w_{F}\left(p_{-}, 1\right)+\sum_{i=1}^{N} w_{G}\left(k_{i}, \Theta_{i}\right)\right],
$$

where $\Theta_{i}$ is the angle between gluon $i$ and its parent:

$$
\vec{k}_{i} \overrightarrow{\mathcal{P}}_{i}=\left|\vec{k}_{i}\right|\left|\overrightarrow{\mathcal{P}}_{i}\right| \cos \Theta_{i} .
$$

This factor, depending on the topology of $\mathcal{D}$, is closely connected with the radiation cross section (5.21). Namely, function $w_{G}(F)$ describes the total probability of soft gluon emission off a gluon (quark) inside a cone of halfangle $\Theta$ :

$$
w_{G}(p, \Theta)=\int_{\Gamma(p, \Theta)} \frac{g_{s}^{2}}{(2 \pi)^{3}} N_{c} \frac{d^{3} k}{2 \omega} \frac{\vec{p}^{2} \sin ^{2} \Theta_{k}}{(k p)^{2}} \approx \int_{\Gamma(p, \Theta)} d \mathcal{K}(\vec{k}) .
$$

The total probability of a quark $(F)$ decay inside the restricted angular cone $w_{F}$ in (5.24) is proportional in DLA to $w_{G}$ :

$$
w_{F}(p, \Theta) \approx \frac{C_{F}}{N_{c}} w_{G}(p, \Theta) .
$$

The multiplicand $\mathcal{F}^{2}$ appearing in the cross section is nothing but the product of two quark and $N$ gluonic QCD Form Factors (see Section 1.2.4). The 
variables $\Theta_{i}$ in (5.24) can be said to represent the "jet opening angles", the angular phase space available for subsequent development of independent subjets generated by partons $i=1 \ldots N$. For two primary quarks this evolution parameter is the largest and was taken to be unity in (5.24) since a more accurate fixing of its value (e.g., $\pi$ or so) would be outside DLA accuracy.

The ansatz (5.24) for the account of virtual contributions to the tree multigluon amplitudes (5.17) made in early papers on the subject 2213 was based on the low order PT calculations and ones physical intuition. The formal proof was given later in Ref. [4 where the Gribov Bremsstrahlung theorem 5 based on the dispersion considerations and the Kirshner-Lipatov method of constructing the Bethe-Salpeter-type equations for PT amplitudes [6 had been exploited. Similar structure appears in the framework of general approach of asymptotic dynamics to coherent QCD states (see [7] and references therein).

\subsection{Method of Generating Functionals}

Generating Functionals (GF), first applied to a description of QCD partonic systems in Jet Calculus by Konishi, Ukawa and Veneziano in 1979 [ direct generalization of the well known mathematical object, namely Generating Functions. The notion of Generating Function have being exploited since Euler times to replace the study of the important numerical series by an analysis of functions "generating" them.

For example, the Taylor expansion for $G(u)=\exp (u)$ can be said to "generate" $a_{n}=1$ series according to the expansion

$$
\left.a_{n} \equiv\left(\frac{d}{d u}\right)^{n} G(u)\right|_{\{u=0\}}
$$




$$
\begin{aligned}
& G(u)=u \exp (u) \quad \Longrightarrow \quad 0,1,2 \ldots n \ldots \text { natural numbers, } \\
& u /\left(e^{u}-1\right) \quad \Longrightarrow B_{n} \quad \text { Bernulli series, } \\
& G(u ; x)=\exp \left(2 x u-u^{2}\right) \Longrightarrow H_{n}(x) \quad \text { Hermite polinomials, } \\
& \text { etc., etc. }
\end{aligned}
$$

Following the same logic one can consider our $N$-gluon production cross section as the $N$-th term in a series expansion of some "generating" object which accumulates the overall QCD cascade picture. This object now must be not a function but a functional since the series, it has to generate, consists of functions (i.e. depending on $N$ 3-dimensional gluon momenta) and not of numbers any more.

\subsubsection{Exclusive and Inclusive Cross Sections in GF Technique}

We are now going to replace the exclusive $N$-gluon cross section of (5.20) (with (5.22) standing for its symbolic expression) by the functional $d \sigma\{u\}$ which will generate $d \sigma_{N}$ as the "coefficients" in the Taylor expansion of $\sigma\{u\}$ with respect to the "probing functions" $u(k)$ :

$$
d \sigma_{N}^{e x c l}=\left.\left(\prod_{i=1}^{N} d^{3} k_{i} \frac{\delta}{\delta u\left(k_{i}\right)}\right) d \sigma\{u\}\right|_{\{u=0\}} .
$$

To obtain this functional we must simply sum up all tree contributions weighted with the arbitrary functions $u\left(k_{i}\right)$ and integrated over final gluon momenta $k_{i}$ :

$$
d \sigma\{u\}=d \sigma_{0} \sum_{N=0,1 \ldots \infty} \mathcal{F}^{2} \prod \int_{\Gamma(\mathcal{P}(k), \Theta(\mathcal{P}))} d \mathcal{K}(\vec{k}) * u(k) .
$$


Equation (5.27) comes immediately from the construction of (5.28) after making use of the definition of the variational derivative:

$$
\left(\frac{\delta}{\delta u\left(k_{i}\right)}\right) u(k) \equiv \delta^{3}\left(\vec{k}_{i}-\vec{k}\right) .
$$

The notion of GF appears to be very convenient for studying inclusive particle distributions. To get the $N$-parton inclusive cross section one has to apply to $d \sigma\{u\}$ the operator

$$
\begin{aligned}
d \sigma_{N}^{i n c l}= & \left.\left(\prod_{i=1}^{N} d^{3} k_{i} \frac{\delta}{\delta u\left(k_{i}\right)}\right) \sum_{m=0}^{\infty} \frac{1}{m !}\left(\prod_{j=1}^{m} d^{3} k_{j} \frac{\delta}{\delta u\left(k_{j}\right)}\right) d \sigma\{u\}\right|_{\{u=0\}} \\
& =\left.\left(\prod_{i=1}^{N} d^{3} k_{i} \frac{\delta}{\delta u\left(k_{i}\right)}\right) \exp \left\{\int d^{3} k \frac{\delta}{\delta u(k)}\right\} d \sigma\{u\}\right|_{\{u=0\}} \\
& =\left.\left(\prod_{i=1}^{N} d^{3} k_{i} \frac{\delta}{\delta u\left(k_{i}\right)}\right) d \sigma\{u\}\right|_{\{u=1\}}
\end{aligned}
$$

which proves to be equivalent to the functional expansion near the "point" $u(k) \equiv 1$. The last thing to be mentioned in this paragraph is that due to independent evolution of the primary $q$ and $\bar{q}$ one can represent (5.28) as a product of two GFs responsible for the development of a quark jet:

$$
d \sigma^{e^{+} e^{-}}\{u\}=d \sigma_{0} Z_{F}\left(p_{+}, 1 ;\{u\}\right) Z_{F}\left(p_{-}, 1 ;\{u\}\right) .
$$

\subsubsection{Generating Functional (GF)s for $g$ and $q$ Jets}

Now it is time to invoke our knowledge of the structure of AO tree graphs to derive the expression for jet GFs. A good way is to guess the answer first and then give the formal proof. 
Constructing GF. Take a lone gluon $l$ which itself produces no softer radiation and is always there in any nonempty tree $(N>0)$. Its personal GF is trivial and describes a subjet consisting of a single primary particle :

$$
Z\left(l, \Theta_{l} ;\{u\}\right)=u(l) e^{-w_{G}\left(l, \Theta_{l}\right)}=u(l) \exp \left\{\int_{\Gamma\left(l, \Theta_{l}\right)} d \mathcal{K} \cdot[-1]\right\} .
$$

$\Theta_{l}$ here is the emission angle of $l$ which plays the role of the opening angle of the $l$-jet. The Form Factor $w_{G}$ which our gluon brings to the cross section according to (5.22) (5.25) is represented in (5.31) in a somewhat peculiar form for future use.

Now we proceed with a gluon which radiates a few "lonely" gluons. Let its momentum be $k$, production angle $\Theta$ and those of its sequential offsprings $-l_{1}, \ldots l_{m}$ and $\Theta_{1}>\Theta_{2}>\ldots>\Theta_{m}$.

$$
\begin{aligned}
& Z(k, \Theta ;\{u\})=u(k) e^{-w_{G}(k, \Theta)} \int_{\Gamma(k, \Theta)} d \mathcal{K}\left(\vec{l}_{1}\right) u\left(l_{1}\right) e^{-w_{G}\left(l_{1}, \Theta_{1}\right)} \times \\
& \quad \times \int_{\Gamma\left(k, \Theta_{1}\right)} d \mathcal{K}\left(\vec{l}_{2}\right) u\left(l_{2}\right) e^{-w_{G}\left(l_{2}, \Theta_{2}\right)} \ldots \int_{\Gamma\left(k, \Theta_{m-1}\right)} d \mathcal{K}\left(\vec{l}_{m}\right) u\left(l_{m}\right) e^{-w_{G}\left(l_{m}, \Theta_{m}\right)} .
\end{aligned}
$$

All the integrands here are identical (no energy ordering among $l_{i}$ !) so one can take off the angular restrictions replacing (5.32) by

$$
Z(k, \Theta ;\{u\})=u(k) e^{-w_{G}(k, \Theta)} \frac{1}{m !}\left[\int_{\Theta>\Theta_{l}} d \mathcal{K}(\vec{l}) Z\left(l, \Theta_{l} ;\{u\}\right)\right]^{m}
$$

where we substituted the product $u(l) \exp \left(-w_{G}(l)\right)$ by the GF (5.31) for the "lonely" gluon. Now, after summation over $m=0 \ldots \infty$ and making use of the tricky representation of the $\exp \left(-w_{G}\right)$ factor we have demonstrated above 
in (5.31), one finally arrives at the following construction:

$$
\begin{aligned}
& Z(k, \Theta ;\{u\})=u(k) \exp \left(\int_{\Gamma(k, \Theta)} d \mathcal{K}(\vec{\ell})\left[Z\left(\ell, \Theta_{\ell} ;\{u\}\right)-1\right]\right) \\
& \quad \equiv u(k) \exp \left(\int_{\Gamma(k, \Theta)} \frac{d \ell}{\ell} \frac{d^{2} \vec{\ell}_{\perp}}{2 \pi \ell_{\perp}^{2}} \frac{2 N_{c} \alpha_{s}}{\pi}\left[Z\left(\ell, \Theta_{\ell} ;\{u\}\right)-1\right]\right)
\end{aligned}
$$

The $Z$-functional in the exponent represented till now the primitive "empty" jets, however (5.32) looks too nice not to hold in general. But instead of following the line of consecutive complications (i.e. considering jets built up of jets consisting of "lonely" gluons etc.) we should feel ourselves educated enough at the moment to be able to prove that (5.32) is indeed the Master Equation for the GF of gluon jet in DLA.

Bethe-Salpeter Equation for $Z_{G}$ and $Z_{F}$. The main property of a tree which may serve as the definition of this object (and it does in mathematics) is that it consists of a node and two more trees 1. In our problem such a recursive definition means that to describe the jet it is enough, in fact, to describe its first decay:

$$
\begin{aligned}
& Z_{A}(k, \Theta ;\{u\})=u(k) e^{-w_{A}(k, \Theta)}+ \\
& \int_{\Gamma(k, \Theta)} \frac{d \omega^{\prime}}{\omega^{\prime}} \frac{d^{2} k_{\perp}^{\prime}}{k_{\perp}^{\prime 2}} \frac{2 C_{A} \alpha_{s}}{\pi} e^{-w_{A}(k, \Theta)+w_{A}\left(k, \Theta^{\prime}\right)} Z_{A}\left(k, \Theta^{\prime} ;\{u\}\right) Z_{G}\left(k^{\prime}, \Theta^{\prime} ;\{u\}\right) .
\end{aligned}
$$

A few comments are to be made.

\footnotetext{
${ }^{1}$ To be precise, this saying defines a binary tree
} 
- We have introduced a new subscript $A$ to match both $g$ and $q$ jet GFs $(A=G, F)$.

- We are already familiar with the first term on the r.h.s. describing the "bare" jet ( $c f$. (5.31) $)$.

- The integral term displays emission of a soft gluon $k^{\prime}=\left(\omega^{\prime}, \vec{k}^{\prime}\right)$ off $A$ (proportional to corresponding color factor $C_{A}$ ), which is followed by subsequent evolution of two jets $G$ and $A$ with momenta $k^{\prime}$ and $k$ (recoil effect neglected in DLA!) and, by virtue of the AO rules, opening angles $\Theta^{\prime}$.

- DLA integration condition $\Gamma$ of (5.23) states

$$
\omega^{\prime}<k^{0}=|\vec{k}| \quad, \quad \Theta^{\prime}<\Theta
$$

where $\Theta$ is an external parameter of the primary jet (emission angle of $A=G$ or $\Theta \sim 1$ for initial quark $A=F$ ).

- The exponential factor under the integral ensures that the decay we are considering is the first one: it is the probability for parton $A$ to stay as a bare particle in the interval of radiation angles between $\Theta^{\prime}$ and $\Theta$.

Making iterations of the integral equation (5.32), i.e. substituting consecutively the Born term under the integral, one can check that it reproduces indeed all AO trees. The evolution parameter $\Theta$ is present only in the external jet Form Factor and as an upper limit of the $\Theta^{\prime}$ integration.

$$
\int_{\Gamma(k, \Theta)} \frac{d \omega^{\prime}}{\omega^{\prime}} \frac{d^{2} k_{\perp}^{\prime}}{k_{\perp}^{\prime 2}}=\int_{Q_{0} / k}^{\Theta} \frac{d \Theta^{\prime}}{\Theta^{\prime}} \int_{Q_{0} / \Theta^{\prime}}^{k} \frac{d \omega^{\prime}}{\omega^{\prime}} \int_{0}^{2 \pi} \frac{d \phi^{\prime}}{2 \pi}
$$


This observation allows one to solve (5.32) by taking $\Theta$-derivative of the product $Z \exp (-w)$ :

$$
\frac{\partial}{\partial \ln \Theta}\left[e^{-w_{A}(k, \Theta)} Z_{A}(k, \Theta)\right]=e^{-w_{A}(k, \Theta)} \int_{Q_{0} / \Theta}^{k} \frac{d \omega^{\prime}}{\omega^{\prime}} \int_{0}^{2 \pi} \frac{d \phi^{\prime}}{2 \pi} \frac{2 C_{A} \alpha_{s}}{\pi} Z_{A}(k, \Theta) Z_{G}\left(k^{\prime}, \Theta\right) .
$$

Now, making use of (5.25), (5.26) for gluon emission probabilities to get the $\Theta$-derivative of the parton Form Factors on the l.h.s., one obtains

$$
\frac{\partial}{\partial \ln \Theta} Z_{A}(k, \Theta)=Z_{A}(k, \Theta) \int_{Q_{0} / \Theta}^{k} \frac{d \omega^{\prime}}{\omega^{\prime}} \int_{0}^{2 \pi} \frac{d \phi^{\prime}}{2 \pi} \frac{2 C_{A} \alpha_{s}}{\pi}\left[Z_{G}\left(k^{\prime}, \Theta\right)-1\right] .
$$

Integrating the expression for $\frac{\partial}{\partial \ln \Theta} \ln Z_{A}$ with an obvious initial condition for the very start of PT jet evolution

$$
\left.Z_{A}(k, \Theta ;\{u\})\right|_{k \Theta=Q_{0}}=u(k),
$$

one immediately arrives at the generalized Master Equation (5.32) which we guessed in the previous subsection for the gluon jet:

$$
Z_{A}(p, \Theta ;\{u\})=u(p) \exp \left(\int_{\Gamma(p, \Theta)} \frac{d \omega}{\omega} \frac{d^{2} k_{\perp}}{2 \pi k_{\perp}^{2}} \frac{C_{A}}{N_{c}} \gamma_{0}^{2}\left[Z_{G}\left(k, \Theta_{k} ;\{u\}\right)-1\right]\right)
$$

Here we have introduced a shorthand notation for the color charge factor depending on $k_{\perp}$ of the parton:

$$
\gamma_{0}^{2}=4 N_{c} \frac{\alpha_{s}\left(k_{\perp}^{2}\right)}{2 \pi}=\frac{4 N_{c}}{b \ln \left(k_{\perp} / \Lambda\right)} \quad, \quad b=\frac{11}{3} N_{c}-\frac{2}{3} n_{f} .
$$


Notice, that at $u(k) \equiv 1$, which is an important point in the functional space for deriving inclusive jet characteristics, (5.35) gives

$$
\left.Z_{A}(p, \Theta ;\{u\})\right|_{\{u=1\}} \equiv 1 .
$$

This property makes the total cross section free of logarithmic PT divergencies and thus of $Q_{0}$-dependence. Indeed, according to (5.29), (5.30) the total inclusive $e^{+} e^{-}$annihilation cross section $(N=0$ registered particles) reads

$$
d \sigma_{\text {tot }}^{e^{+} e^{-}}=\left.d \sigma^{e^{+} e^{-}}\{u\}\right|_{\{u=1\}}=\left.d \sigma_{0} Z_{F}\left(p_{+}, 1 ;\{u\}\right) Z_{F}\left(p_{-}, 1\{u\}\right)\right|_{\{u=1\}}=d \sigma_{0} .
$$

Note, that the GFs similar to (5.30) can be easily constructed (by means of the basic GF for a gluon jet) for any other initial partonic state $(\rho)$ consisting of $n_{q}$ quarks and $n_{g}$ gluons with energies of the same order $p_{i} \sim p$ and large relative angles $\Theta_{i j} \sim 1$

$$
\begin{array}{cl}
d \sigma^{(\rho)}\{u\}=d \sigma_{0}\left[Z_{G}(p, 1 ;\{u\})\right]^{\rho} \quad, \quad \rho=\frac{C_{F}}{N_{c}} n_{q}+n_{g}: \\
e^{+} e^{-} \rightarrow q \bar{q} & \rho=2 C_{F} / N_{c}=8 / 9 \\
e^{+} e^{-} \rightarrow q \bar{q} g & \rho=2 C_{F} / N_{c}+1=17 / 9 \\
\Upsilon \rightarrow g g g & \rho=3, \quad \text { etc. }
\end{array}
$$

\subsection{Multiplicity Distributions in QCD Jets}

In this section we apply the GF technique to consider the mean parton multiplicity and character of multiplicity fluctuations in QCD jets following Refs. 910. There are two complementary ways of dealing with multiplicity fluctuations: studying the distribution $P_{n}=\sigma^{(n)} / \sigma$ of events over the 
number of produced particles and/or measuring inclusive multiplicity correlators $n_{k} \equiv\langle n(n-1) \ldots(n-k+1)\rangle$. According to (5.27) which defines the exclusive characteristics of parton distributions in terms of GF, the total probability of $n$-particle production reads

$$
P_{n}=\left.\frac{1}{n !} \prod_{i=1}^{n}\left(\int d^{3} k_{i} \frac{\delta}{\delta u\left(k_{i}\right)}\right) Z(\{u\})\right|_{\{u=0\}} .
$$

A variational derivative over the probing functions $u(k)$ followed by an integration over the whole phase space of particle momenta proves to be equivalent to simple differentiation of the function $Z(u), u(k) \equiv u$ :

$$
\begin{gathered}
P_{n}(y)=\left.\frac{1}{n !}\left(\frac{d}{d u}\right)^{n} Z(y ; u)\right|_{\{u=0\}} . \\
Z(y ; u)=\sum_{n=0}^{\infty} P_{n}(y) u^{n}
\end{gathered}
$$

Replacing $u(k)$ by a constant in the Master Equation (5.35), one can simply check that its solution appears to depend not on the jet energy and its opening angle separately, but on their product, i.e. on the characteristic transverse momentum of the jet

$$
Z(p, \Theta ; u)=Z(y ; u), \quad y=\ln \frac{p \Theta}{Q_{0}} \equiv \ln \frac{Q}{Q_{0}} .
$$

Performing a partial integration in (5.35) with use of the following chain of transformations

$$
\begin{aligned}
\int_{\Gamma(p, \Theta)} \frac{d \omega}{\omega} \frac{d^{2} k_{\perp}}{2 \pi k_{\perp}^{2}} & =\int^{p} \frac{d \omega}{\omega} \int^{\Theta} \frac{d \Theta_{k}}{\Theta_{k}} \vartheta\left(\omega \Theta_{k}-Q_{0}\right) \\
=\int_{Q_{0} / \Theta}^{p} \frac{d \omega}{\omega} \int_{Q_{0}}^{\omega \Theta} \frac{d\left(\omega \Theta_{k}\right)}{\omega \Theta_{k}} & =\int_{\ln Q_{0}}^{\ln (p \Theta)} d \ln (\omega \Theta) \int_{\ln Q_{0}}^{\ln (\omega \Theta)} d \ln k_{\perp}
\end{aligned}
$$


one arrives at the simplified Master Equation for GF of gluon jet:

$$
Z(y ; u)=u \exp \left(\int_{0}^{y} d y^{\prime}\left(y-y^{\prime}\right) \gamma_{0}^{2}\left(y^{\prime}\right)\left[Z\left(y^{\prime} ; u\right)-1\right]\right), \quad y^{\prime} \equiv \ln \frac{k_{\perp}}{Q_{0}} .
$$

The initial and normalization conditions, (5.34) and (5.37), now read

$$
\begin{aligned}
& Z(0 ; u)=u \\
& Z(y ; 1)=1 .
\end{aligned}
$$

Notice, that due to (5.42) the multiplicity distribution is normalized to unity:

$$
\sum_{n=0}^{\infty} P_{n}=\left.\sum_{n=0}^{\infty} \frac{1}{n !}\left(\frac{d}{d u}\right)^{n} Z(u)\right|_{\{u=0\}}=\left.e^{d / d u} Z(u)\right|_{\{u=0\}}=\left.Z(u)\right|_{\{u=1\}}=1 .
$$

Multiplicity Correlators can be calculated by means of the $P_{n}$ distribution as follows:

$$
n_{k}(y) \equiv \sum_{n=k}^{\infty} n(n-1) \ldots(n-k+1) P_{n}(y) .
$$

This procedure is equivalent to calculating the $k^{\text {th }}$ term of the Taylor expansion for $Z$ at $u=1$

$$
n_{k}(y)=\left.\left[\left(\frac{d}{d u}\right)^{k} \sum_{n=0}^{\infty} u^{n} P_{n}(y)\right]\right|_{\{u=1\}}=\left.\left(\frac{d}{d u}\right)^{k} Z(y ; u)\right|_{\{u=1\}}
$$


Therefore an alternative expansion series for GF near $u=1$ could be written in terms of multiplicity correlators:

$$
Z(y ; u)=\sum_{k=0}^{\infty} \frac{(u-1)^{k}}{k !} n_{k}(y), \quad\left(n_{0} \equiv 0 ; \quad c f .(\underline{5.43})\right) .
$$

Mean Multiplicity of partons corresponds to $k=1$ in (5.44): $\langle n\rangle=$ $n_{1} \equiv \bar{n}$. Differentiating (5.40) over $u$ and putting $u=1, Z=1$, one gets the DLA equation for $\bar{n}(y)$

$$
\bar{n}(y)=1+\int_{0}^{y} d y^{\prime}\left(y-y^{\prime}\right) \gamma_{0}^{2}\left(y^{\prime}\right) \bar{n}\left(y^{\prime}\right) .
$$

Differentiating over $y$ one has

$$
\begin{aligned}
\bar{n}^{\prime}(y) & =\int_{0}^{y} d y^{\prime} \gamma_{0}^{2}\left(y^{\prime}\right) \bar{n}\left(y^{\prime}\right) \\
\bar{n}^{\prime \prime}(y) & =\gamma_{0}^{2}(y) \bar{n}(y)
\end{aligned}
$$

with initial conditions

$$
\bar{n}(0)=1, \quad \bar{n}^{\prime}(0)=0 .
$$

The solution for constant color coupling would be simply

$$
\bar{n}(y)=\cosh \left(\gamma_{0} y\right) \quad \approx \frac{1}{2} e^{\gamma_{0} y}=\left(\frac{Q}{Q_{0}}\right)^{\sqrt{2 N_{c} \alpha_{s} / \pi}} .
$$

With an account of running $\alpha_{s}$, (5.48) can be reduced to the differential equation for modified Bessel functions, the solution of which satisfying initial conditions (5.49) reads

$$
\bar{n}(y)=A \sqrt{Y}\left\{I_{1}(A \sqrt{Y}) K_{0}(A \sqrt{\lambda})+K_{1}(A \sqrt{Y}) I_{0}(A \sqrt{\lambda})\right\} ;
$$




$$
Y \equiv y+\lambda=\ln (p \Theta / \Lambda), \quad A \equiv \sqrt{\frac{16 N_{c}}{b}} .
$$

The PT approach implies $\alpha_{s}\left(Q_{0}\right) / \pi \ll 1$, i.e. $\lambda \gg 1$, therefore one can use asymptotic expressions for modified Bessel functions 11$]$

$$
I_{\nu}(z) \approx \frac{1}{\sqrt{2 \pi z}} e^{z}, \quad K_{\nu}(z) \approx \sqrt{\frac{\pi}{2 z}} e^{-z}
$$

to obtain

$$
\bar{n}(y) \approx\left(\frac{Y}{\lambda}\right)^{1 / 4} \cosh (A(\sqrt{Y}-\sqrt{\lambda})) \sim \exp \sqrt{\frac{16 N_{c}}{b} \ln \frac{Q}{\Lambda}} .
$$

The preexponential factor here should not be taken seriously, since, as we shall see in the next Chapter, a number of subleading effects, such as an account of sea-quark contribution to gluonic cascades, recoil effect etc., will modify the power of $\alpha_{s}\left(Q_{0}\right) / \alpha_{s}(Q)$ ratio.

Notice the peculiar energy behavior of the mean multiplicity of partons in QCD jet given by (5.53): it grows slower than a power of energy $Q$, but faster than any power of $\ln Q$.

Both constant (5.50) and running coupling (15.53) cases are matched within the DLA accuracy by the common expression

$$
\bar{n}(y)=\exp \left\{\int^{y} d y^{\prime} \gamma_{0}\left(y^{\prime}\right)\right\}
$$

which is nothing but the WKB approximation to (5.48). The quantity $\gamma_{0}=\gamma_{0}\left(\alpha_{s}\right)$ defined by (5.36) thus can be said to represent the anomalous dimension determining the rate of multiplicity growth with energy. 


\subsection{KNO Phenomenon}

As it is well known, the KNO phenomenon ${ }^{[12]}$ means the dependence of the multiplicity distribution on the total energy $y=\ln Q$ only via the ratio $x=n / \bar{n}(y)$. This kind of scaling behavior in hard processes was discovered in field theory by Polyakov in $1971^{[13]}$. To be more precise, the KNO distribution is defined as

$$
\bar{n}(y) P_{n}(y)=f(x) \quad, \quad x \equiv n / \bar{n}(y) .
$$

QCD jets exhibit exact KNO scaling in the asymptotics $y \rightarrow \infty$. To prove this statement we must express it in terms of GF. Since typical values of $n$ grow with $y$, one can approximate the sum in (5.39) by an integral:

$$
Z(y ; u)=\sum_{n=0}^{\infty} P_{n} u^{n} \approx \int_{0}^{\infty} \frac{d n}{\bar{n}}\left[\bar{n} P_{n}\right] u^{n}=\int_{0}^{\infty} d x f(x)\left(u^{\bar{n}}\right)^{x} .
$$

To make the r.h.s. depend on $x$ alone, one must choose $u^{\bar{n}}$ to be constant. For that let us take

$$
\begin{gathered}
u=\exp \left(-\frac{\beta}{\bar{n}}\right) \quad, \quad \beta=\text { const } . \\
\Phi(\beta) \equiv \int_{0}^{\infty} d x f(x) e^{-\beta x}
\end{gathered}
$$

Thus, the asymptotic KNO scaling in QCD exists with the limit

$$
\lim _{y \rightarrow \infty} Z\left(y ; e^{-\beta / \bar{n}(y)}\right)=\Phi(\beta) .
$$

Constructing $\Phi(\beta)$ with a help of alternative series of (5.45), one obtains

$$
\Phi(\beta)=\lim _{y \rightarrow \infty} \sum_{k=0}^{\infty} \frac{1}{k !}\left(e^{-\beta / \bar{n}}-1\right)^{k} n_{k}=\sum_{k=0}^{\infty} \frac{(-\beta)^{k}}{k !} \lim _{y \rightarrow \infty}\left[\frac{n_{k}(y)}{(\bar{n}(y))^{k}}\right] .
$$


Expanding the exponent in (5.56) we have

$$
\Phi(\beta)=\int_{0}^{\infty} d x f(x) \sum_{k=0}^{\infty} \frac{(-\beta x)^{k}}{k !}=\sum_{k=0}^{\infty} \frac{(-\beta)^{k}}{k !} f_{k}
$$

Comparing (5.58) with (5.59) one concludes that the normalized multiplicity correlators are nothing but the moments of the KNO function of (5.56):

$$
f_{k} \equiv \int_{0}^{\infty} d x x^{k} f(x)=\lim _{y \rightarrow \infty}\left[\frac{n_{k}(y)}{(\bar{n}(y))^{k}}\right]
$$

The first two coefficients in the power series (15.59) for $\Phi$ are fixed:

$$
f_{0}=\int_{0}^{\infty} d x f(x)=1 \quad, \quad f_{1}=\int_{0}^{\infty} d x x f(x)=1 .
$$

Having in hand the $\Phi(\beta)$ function one can reconstruct the KNO function with use of an inverse Mellin transformation:

$$
f(x)=\int \frac{d \beta}{2 \pi i} \Phi(\beta) e^{\beta x}
$$

where the integral runs parallel to the imaginary axis to the right of the singularities of $\Phi$ in the complex $\beta$-plane.

To find the function $\Phi(\beta)$ one has either to calculate $f_{k}, k=2, \ldots \infty$ or invoke the Evolution Equation (5.40) for the direct search for the $y \rightarrow \infty$ limit in (5.57). We shall trace both ways to obtain recurrency relations for multiplicity moments and to derive analytical properties of $\Phi(\beta)$ for getting knowledge about asymptotic behavior of KNO distribution $(x \rightarrow 0$ and $x \gg 1)$. 


\subsubsection{Recurrency Relations for Multiplicity Moments}

Let us differentiate (5.40) over $y$ to obtain

$$
Z^{\prime}(y ; u)=Z(y ; u) \int_{0}^{y} d y^{\prime} \gamma_{0}^{2}\left(y^{\prime}\right)\left[Z\left(y^{\prime} ; u\right)-1\right]
$$

Then substituting expansion (5.45) for $Z$ and making the ansatz $n_{k}(y)=$ $(\bar{n}(y))^{k} \cdot f_{k}$, collect terms proportional to $(u-1)^{k}$. The result will be

$$
\begin{array}{r}
\frac{d \bar{n}(y)}{d y}=\int_{0}^{\infty} d y^{\prime} \gamma_{0}^{2}\left(y^{\prime}\right) \bar{n}\left(y^{\prime}\right) \quad \text { for } k=1, \\
\frac{k f_{k}}{k !} \bar{n}(y)^{k-1} \frac{d \bar{n}(y)}{d y}=\sum_{m=1}^{k} \frac{f_{k-m} f_{m}}{(k-m) ! m !} \bar{n}(y)^{k-m} \int_{0}^{y} d y^{\prime} \gamma_{0}^{2}\left(y^{\prime}\right) \bar{n}\left(y^{\prime}\right)^{m} .
\end{array}
$$

Equation (5.63a) is equivalent to (5.47) and reproduces once again the energy behavior of the total multiplicity (5.54). It certainly depends on the coupling $\gamma_{0}^{2}$. This, however, is not the case for the KNO distribution. Indeed, estimating the integral term in (5.63b) as

$$
\int_{0}^{y} d y^{\prime} \gamma_{0}^{2} \cdot\left(\bar{n}\left(y^{\prime}\right)\right)^{m}=\frac{1}{m}(\bar{n}(y))^{m-1} \int_{0}^{y} d y^{\prime} \gamma_{0}^{2} \cdot \bar{n}\left(y^{\prime}\right)\left[1+O\left(\frac{1}{\ln \bar{n}}\right)\right]
$$

and making use of (5.63a) we come to the recurrency relations

$$
\frac{k}{k !} f_{k}=\sum_{m=1}^{k} \frac{f_{k-m} f_{m}}{(k-m) ! m !} \frac{1}{m}=\frac{1}{k k !} f_{k}+\sum_{m=1}^{k-1} \frac{f_{k-m} f_{m}}{(k-m) ! m !} \frac{1}{m},
$$




$$
f_{k}=\frac{k}{k^{2}-1} \sum_{m=1}^{k-1} C_{k}^{m} \frac{f_{m} f_{k-m}}{m}=\frac{k^{2}}{2\left(k^{2}-1\right)} \sum_{m=1}^{k-1} C_{k}^{m} \frac{f_{m} f_{k-m}}{m(k-m)},
$$

where

$$
C_{k}^{m} \equiv \frac{k !}{m !(k-m) !} \quad, \quad f_{0}=f_{1}=1 .
$$

Following the same lines for a general case $\rho \neq 1$ one obtains

$$
\begin{gathered}
f_{k}^{(\rho)}=\frac{\rho^{1-k}}{k^{2}} f_{k}+\frac{1}{k} \sum_{m=1}^{k-1} C_{k}^{m} \frac{\rho^{1-m}}{m} f_{m} f_{k-m}^{(\rho)}, \\
f_{0}^{(\rho)}=f_{1}^{(\rho)}=1 .
\end{gathered}
$$

These relations contain no memory about the coupling at all. This means that the QCD KNO function $f(x)$ remains the same for $\alpha_{s}$ being running or fixed, $\gamma_{0}^{2}$ being smaller or larger.

This amusing phenomenon which had been noticed by Bassetto, Ciafaloni and Marchesini in Ref. [14] means that the distribution of multiplicity fluctuations (measured in units of the mean multiplicity $\bar{n}(y)$ ) are determined totally by the very character of $1 \rightarrow 2$ parton cascading, being insensitive to "details" of QCD branching, such as the value and the energy behavior of $\alpha_{s}$ , the number of colors $N_{c}$ etc.

The recurrency relations (5.64), (5.65) could be converted to compact nonlinear integral equations for KNO functions ${ }^{[10]}$ :

$$
\begin{aligned}
x f(x) & =\int_{0}^{x} d y f(x-y) \int_{y}^{\infty} d t f(t) \ln \frac{t}{y}, \\
x f^{(\rho)}(x) & =\int_{0}^{\rho x} d y f^{(\rho)}\left(x-\frac{y}{\rho}\right) \int_{y}^{\infty} d t f(t) \ln \frac{t}{y} .
\end{aligned}
$$


Let us remind the reader that $f(x)$ describes the asymptotic KNO distribution in a single gluon jet and $f^{(\rho)}(x)$ corresponds to an arbitrary jet ensemble according to (15.38).

\subsubsection{Analytic Solution of the KNO Problem}

Turning back to the definition of the Mellin transformed KNO function (5.56), (5.57) we can now simplify the problem keeping $\gamma_{0}$ fixed $\left(\alpha_{s}\left(y^{\prime}\right)=\right.$ const). Differentiating (5.62) over $y$ once more one has

$$
(\ln Z(y))^{\prime \prime}=\gamma_{0}^{2}(Z(y)-1) \quad ; \quad Z(0)=u \quad, \quad Z^{\prime}(0)=0 .
$$

The first integral of this differential equation reads

$$
\frac{1}{2}(\ln Z)^{\prime 2}=\gamma_{0}^{2} \int^{Z} \frac{d Z^{\prime \prime}}{Z}\left(Z^{\prime}-1\right)=\gamma_{0}^{2}(Z-\ln Z+\text { const }) .
$$

The initial conditions of (5.67) determine the constant of integration to be equal to $-u+\ln u$ :

$$
\pm(\ln Z)^{\prime}=\gamma_{0} \sqrt{2\left(Z-u-\ln \frac{Z}{u}\right)} .
$$

Introducing $x=Z / u$ one gets the solution of (5.68)

$$
\begin{aligned}
\gamma_{0} \cdot y & =\int_{Z / u}^{1} \frac{d x}{x \sqrt{2[u(x-1)-\ln x]}}, \quad u \leq 1, \\
\gamma_{0} \cdot y & =\int_{1}^{Z / u} \frac{d x}{x \sqrt{2[u(x-1)-\ln x]}}, \quad u \geq 1 .
\end{aligned}
$$


The upper (lower) limit $x=1$ here comes out from the initial condition $Z=u$ at $y=0$. Now we have to substitute $u=\exp (-\beta / \bar{n}(y))$ and consider the limit $y \rightarrow \infty$. The integral diverges logarithmically when $u \rightarrow 1$. For $u \leq 1$, e.g., one has

$$
\int_{1 \in \epsilon}^{1} \frac{d x}{x \sqrt{2[u(x-1)-\ln x]}} \approx \int \frac{d x}{(1-x) \sqrt{1+2 \frac{(1-u)}{(1 x)}}} \approx \ln \frac{\epsilon}{2(1-u)}, 1-u \ll \epsilon \ll 1,
$$

so before taking the limit we must pick up the singular contribution rewriting (5.69a) in the form

$$
\gamma_{0} y=\int_{Z}^{1} \frac{d x}{x}\left(\frac{1}{\sqrt{2(x-1-\ln x)}}-\frac{1}{1-x}\right)+\ln \left[\frac{(1-Z)}{Z} \frac{1}{2(1-u)}\right]
$$

where $u=1$ is taken everywhere except the singular term. Making use of the asymptotic expression (5.50) $\bar{n}(y) \approx \frac{1}{2} e^{\gamma_{0} y}$ one finally obtains the following indirect representations for $\Phi(\beta)$

$$
\begin{aligned}
& \int_{\Phi}^{\frac{1}{2}} \frac{d x}{x \sqrt{2(x-1-\ln x)}}=\ln \frac{\beta}{\beta_{1}}, \quad \beta>0 \\
& \int_{\Phi}^{\infty} \frac{d x}{x \sqrt{2(x-1-\ln x)}}=\ln \frac{\beta_{0}}{-\beta}, \quad \beta<0 .
\end{aligned}
$$

Here we denoted by $\beta_{1}$ and $\beta_{0}$ the positive numbers

$$
\begin{aligned}
& \ln \beta_{1} \equiv \int_{\frac{1}{2}}^{1} \frac{d x}{x}\left(\frac{1}{\sqrt{2(x-1-\ln x)}}-\frac{1}{1-x}\right) \approx-0.251 \\
& \ln \beta_{0} \equiv \int_{1}^{\infty} \frac{d x}{x}\left(\frac{1}{\sqrt{2(x-1-\ln x)}}-\frac{1}{x-1}\right) \approx 0.937
\end{aligned}
$$


Expressions (5.70) solve the differential equation ${ }^{[14]}$, which looks very much alike the original equation (5.67):

$$
\left(\beta \frac{d}{d \beta}\right)^{2} \ln \Phi(\beta)=\Phi(\beta)-1 ;\left.\quad \Phi\right|_{\beta=0}=1,\left.\quad \beta \frac{d}{d \beta} \Phi\right|_{\beta=0}=0
$$

\section{High multiplicity fluctuations}

Behavior of the asymptotic KNO distribution $f(x)$ at large values of $x=n / \bar{n}$ is determined, according to (5.61), by the rightmost singularity of $\Phi(\beta)$ in the $\beta$-plane. As it is clearly seen from (5.70b), $\Phi \rightarrow \infty$ when $\beta \rightarrow-\beta_{0} \approx-2.552$. Expanding $\Phi(\beta)$ near the singularity one obtains

$$
\Phi(\beta)=\frac{2}{z^{2}}+\frac{1}{3} \ln \frac{2}{z^{2}}+\frac{5}{9}+O\left(z^{2}(\ln z)^{2}\right) \approx \frac{2 \beta_{0}^{2}}{\left(\beta+\beta_{0}\right)^{2}}-\frac{2 \beta_{0}}{\beta+\beta_{0}}-\frac{2}{3} \ln \frac{\beta+\beta_{0}}{\beta_{0}}+O(1)
$$

where $z \equiv \ln -\beta_{0} / \beta$. Substituting this expansion into the Mellin integral (5.61), we derive the asymptotic formula for the "tail" of the KNO distribution $f(x)$ at $x \gg 1$ :

$$
f(x)=2 \beta_{0}\left(\beta_{0} x-1+\frac{1}{3 \beta_{0} x}+\ldots\right) e^{-\beta_{0} x} \quad, \quad \beta_{0} x \gg 1 .
$$

The probability of high multiplicity fluctuations decreases with $x$ exponentially. The rate of this fall off does not depend on the type of a hard event under consideration. Indeed, for an arbitrary jet ensemble characterized by the parameter $\rho$ (see (5.38) ) the corresponding KNO function, according to (5.57), would be

$$
f^{(\rho)}(x)=\int \frac{d \beta}{2 \pi i}[\Phi(\beta)]^{\rho} e^{\beta x} .
$$


This relation preserves the position $\beta=-\beta_{0}$ of the singularity. The only change is in its character, which will lead to modification of the preexponential factor for $f^{(\rho)}$ in (5.73).

\section{Low $x$ limit of KNO distribution}

In the opposite limit, when the number of particles produced happens to be much less than the mean multiplicity value $(x \ll 1)$, large $\beta$ dominate where due to (5.70a)

$$
\Phi(\beta) \approx \exp \left(-\frac{1}{2} \ln ^{2} \frac{\beta}{c}\right) \quad, \quad|\beta| \gg 1 .
$$

Evaluating Mellin integral (5.61) one has roughly

$$
f(x) \sim \frac{1}{x} \exp \left(-\frac{1}{2} \ln ^{2} x\right) .
$$

This behavior reminds the form of the DL Form Factor. Though there is no direct correspondence (e.g., (5.75) contains no $\alpha_{s}$ dependence), such a similarity looks physically natural since low multiplicity events originate from undeveloped bremsstrahlung cascades the probability of which is damped by QCD jet Form Factors.

\subsection{Particle Distributions and Correlations}

In the previous Section we made use of the Generating Functional technique to describe asymptotic behavior of multiplicity fluctuations. This device proves to be very useful for the derivation of inclusive energy and angular spectra and correlations of particles as well. 
Later we shall study in details the particle spectra in the framework of a more realistic approach which accounts for essential single-log contributions to parton evolution and makes it possible to predict jet characteristics with controllable accuracy.

DLA provides us with a polygon for learning GF technique. Being too crude for quantitative predictions, DLA, in the same time, can be used for getting rather simple and physically transparent results to demonstrate the main qualitative features of the physics of parton cascades.

In what follows we shall restrict ourselves, for the sake of simplicity, to the case of fixed coupling $\alpha_{s}=$ const.

\subsubsection{Energy Spectrum of Particles in Jet}

Applying the variational derivative to the Master Equation (5.35) one gets the integral equation for the inclusive distribution of partons

$$
\left.\bar{D}_{A}^{p}(x) \equiv E_{p} \frac{\delta}{\delta u\left(k_{p}\right)} Z_{A}\left(E_{A}, \Theta ;\{u\}\right)\right|_{\{u=1\}},
$$

which reads

$$
\bar{D}_{A}^{p}\left(\ell, y_{\Theta}\right)=\delta_{A}^{p} \delta(\ell)+\int_{0}^{\ell} d \ell^{\prime} \int_{0}^{y_{\Theta}} d y^{\prime} \frac{C_{A}}{N_{c}} \gamma_{0}^{2} \cdot \bar{D}_{A}^{p}\left(\ell^{\prime}, y^{\prime}\right) .
$$

Here we introduced the convenient notation:

$$
\begin{aligned}
y_{\Theta} & =\ln \left(k_{p} \Theta / Q_{0}\right), \quad \ell=\ln \left(E_{A} / k_{p}\right) \equiv \ln 1 / x \\
y^{\prime} & =\ln \left(\omega^{\prime} \Theta^{\prime} / Q_{0}\right), \quad \ell^{\prime}=\ln \left(E_{A} / \omega^{\prime}\right)
\end{aligned}
$$


with $\omega^{\prime}, \Theta^{\prime}$ the energy of the intermediate gluon $G$ and its angle with respect to the parent parton. $\Theta$ in (5.76) plays the role of evolution parameter. To obtain the energy distribution of particles in a jet with the total energy $E_{A}$ and the opening angle $\Theta$ one needs to fix the sum

$$
Y_{\Theta} \equiv \ell+y_{\Theta}=\ln \frac{E_{A} \Theta}{Q_{0}} .
$$

Equation (5.76) can be easily solved iteratively:

$$
\bar{D}_{A}^{p}\left(\ell, y_{\Theta}\right)=\delta_{A}^{p} \delta(\ell)+\frac{C_{A}}{N_{c}} \cdot \gamma_{0} \sqrt{\frac{y_{\Theta}}{\ell}} \cdot I_{1}\left(2 \gamma_{0} \sqrt{y_{\Theta} \ell}\right),
$$

where $I_{\nu}$ denotes the standard modified Bessel function.

In particular, for the inclusive energy distribution of particles produced in $e^{+} e^{-}$annihilation (two $q$-jets with $\Theta \sim 1$ ) we have

$$
\frac{d n}{d \ln (1 / x)}=\frac{2 C_{F}}{N_{c}} \cdot \gamma_{0}\left(\frac{\ln \left(E x / Q_{0}\right)}{\ln (1 / x)}\right)^{\frac{1}{2}} \cdot I_{1}\left(2 \gamma_{0} \sqrt{\ln \frac{E x}{Q_{0}} \cdot \ln \frac{1}{x}}\right) .
$$

Spectrum (5.79) exhibits an interesting behavior: it has a maximum at $\ln (1 / x)=\ell=\frac{1}{2} Y$ (so called "hump-backed plateau"). From the point of view of a naive probabilistic picture of parton multiplication one might expect the particle distribution to increase monotonically with $x$ decreasing, since there is more possibilities to get a softer particle from the branching tree.

However, this expectation proves to be wrong: as we discussed in previous Chapter (see Section 4.3) it is the color coherence which prevents multiplication of the softest gluons. 
At large values of $Y=\ln \left(E / Q_{0}\right)$ the Bessel finction in (5.79) can be replaced by its asymptotic expression (5.52) to give

$$
\frac{d n}{d \ell} \propto \exp \left\{2 \gamma_{0} \sqrt{y \ell}\right\} \text {. }
$$

The double-differential distribution of particles is obtained by taking the angle of the registered particle as the jet opening angle $\Theta$ and differentiating (5.78) with respect to it:

$$
\frac{d^{2} n}{d \ln (1 / x) d \ln \Theta}=\frac{C_{A}}{N_{c}} \cdot \gamma_{0}^{2} \cdot I_{0}\left(2 \gamma_{0} \sqrt{\left(Y_{\Theta}-\ln \frac{1}{x}\right) \ln \frac{1}{x}}\right)
$$

\subsubsection{Correlations Between Particles in Jet}

Applying a variational derivative twice with respect to the probing functions $u\left(k_{1}\right)$ and $u\left(k_{2}\right)$ one obtains the double differential inclusive distribution of particles in a jet $A$ :

$$
\frac{1}{\sigma} \frac{\omega_{1} \omega_{2} d^{2} \sigma}{d \omega_{1} d \omega_{2}} \equiv D_{A}^{(2)}\left(E, \Theta ; \omega_{1}, \omega_{2}\right)
$$

All the angular integrations are taken here, and only the energies of two particles $\omega_{i}$ are fixed. The equation for this quantity following directly from (5.35) reads

$$
\begin{array}{r}
D_{A}^{(2)}\left(E, \Theta ; \omega_{1}, \omega_{2}\right)=\frac{C_{A}}{N_{c}} \int \frac{d z}{z} \int \frac{d \Theta^{\prime}}{\Theta^{\prime}} \gamma_{0}^{2}\left\{D_{G}^{(2)}\left(z E, \Theta^{\prime} ; \omega_{1}, \omega_{2}\right)\right. \\
\left.+D_{G}\left(z E, \Theta^{\prime} ; \omega_{1}\right) D_{A}\left(E, \Theta^{\prime} ; \omega_{2}\right)+D_{A}\left(E, \Theta^{\prime} ; \omega_{1}\right) D_{G}\left(z E, \Theta^{\prime} ; \omega_{2}\right)\right\} .
\end{array}
$$


Invoking the DLA equation (5.76) for the one-particle inclusive spectrum one obtains

$$
D_{A}^{(2)}(E, \Theta)-D_{A}\left(E, \Theta ; \omega_{1}\right) D_{A}\left(E, \Theta ; \omega_{2}\right)=\frac{C_{A}}{N_{c}} \int \frac{d z}{z} \int \frac{d \Theta^{\prime}}{\Theta^{\prime}} \gamma_{0}^{2} D_{G}^{(2)}\left(z E, \Theta^{\prime}\right) .
$$

The combination we have on the left hand side of (5.84) is nothing but the correlation function $\widetilde{D}$ which we are aiming at. Equation (5.84) now takes the form

$$
\begin{aligned}
& \widetilde{D}_{A}\left(E, \Theta ; \omega_{1}, \omega_{2}\right)=\frac{C_{A}}{N_{c}} \int \frac{d z}{z} \int \frac{d \Theta^{\prime}}{\Theta^{\prime}} \gamma_{0}^{2} \\
& \quad\left[\widetilde{D}_{G}\left(z E, \Theta^{\prime} ; \omega_{1}, \omega_{2}\right)+D_{G}\left(z E, \Theta^{\prime} ; \omega_{1}\right) D_{G}\left(z E, \Theta^{\prime} ; \omega_{2}\right)\right] .
\end{aligned}
$$

To be definite, let us take $\omega_{1}>\omega_{2}$ and denote by $\eta$ positive quantity

$$
\eta \equiv \ln \frac{\omega_{1}}{\omega_{2}}>0 \text {. }
$$

It is implied that the integrations in (5.83)-(5.85) are limited by the natural restrictions

$$
\omega_{2} \Theta^{\prime}>Q_{0} \quad, \quad z E>\omega_{1} .
$$

Now, introducing

$$
\ell_{1}=\ln \frac{E}{\omega_{1}} \quad \text { and } \quad y_{2}=\ln \frac{\omega_{2} \Theta}{Q_{0}}
$$

as independent variables, we arrive at the following convenient form of (5.85):

$$
\widetilde{D}_{A}\left(\ell_{1}, y_{2}, \eta\right)=\frac{C_{A}}{N_{c}} \int_{0}^{\ell_{1}} d \ell_{1}^{\prime} \int_{0}^{y_{2}} d y_{2}^{\prime} \gamma_{0}^{2}\left[\widetilde{D}_{G}\left(\ell_{1}^{\prime}, y_{2}^{\prime}, \eta\right)+D_{G}\left(\ell_{1}^{\prime}, y_{2}^{\prime}+\eta\right) D_{G}\left(\ell_{1}^{\prime}+\eta, y_{2}^{\prime}\right)\right] .
$$


It is worthwhile to notice that $\ell_{1}$ and $y_{2}$ are less than $\ell_{2}=\ell_{1}+\eta$ and $y_{1}=y_{2}+\eta$ respectively due to the definition of $\eta$.

Let us derive approximately the correlation function $\widetilde{D}$. Noticing that $\bar{D}(\ell, y)(5.78)$ is a monotonically rising function of both its arguments, and using its asymptotic expression (see (5.80) )

$$
\bar{D}(\ell, y) \propto \exp \left(2 \gamma_{0} \sqrt{\ell y}\right)
$$

one can iterate (5.86), evaluating the integrals in (5.86) near the upper limits. Making use of asymptotics (5.87) for the spectra $D_{G}\left(\ell_{1}^{\prime}, y_{2}^{\prime}+\eta\right)$ and $D_{G}\left(\ell_{1}^{\prime}+\right.$ $\left.\eta, y_{2}^{\prime}\right)$, we then expand them near the maxima to get

$$
\begin{gathered}
g^{-1}\left(\ell_{1}, y_{2}, \eta\right) \equiv \frac{1}{D_{G}\left(\ell_{1}, y_{1}\right) D_{G}\left(\ell_{2}, y_{2}\right)} \int_{0}^{\ell_{1}} d \ell_{1}^{\prime} \int_{0}^{y_{2}} d y_{2}^{\prime} \gamma_{0}^{2} D_{G}\left(\ell_{1}^{\prime}, y_{2}^{\prime}+\eta\right) D_{G}\left(\ell_{1}^{\prime}+\eta, y_{2}^{\prime}\right) \\
\approx\left(\sqrt{\frac{y_{1}}{\ell_{1}}}+\sqrt{\frac{y_{2}}{\ell_{2}}}\right)^{-1}\left(\sqrt{\frac{\ell_{1}}{y_{1}}}+\sqrt{\frac{\ell_{2}}{y_{2}}}\right)^{-1}=\frac{1}{2\left[1+\cosh \left(\mu_{1}-\mu_{2}\right)\right]},
\end{gathered}
$$

where we have introduced variables

$$
\mu_{i}=\frac{1}{2} \ln \frac{\ell_{i}}{y_{i}} \quad i=1,2,
$$

"measuring" particle energies with respect to the hump $(\mu=0$ corresponds to the maximum of the inclusive spectrum). The function $g\left(\ell_{1}, y_{2}, \eta\right)$ describes the first iteration of (5.86):

$$
\widetilde{D}_{A}\left(\ell_{1}, y_{2}, \eta\right) \approx \frac{C_{A}}{N_{c}} D_{G}\left(\ell_{1}, y_{1}\right) D_{G}\left(\ell_{2}, y_{2}\right) \cdot g^{-1}\left(\ell_{1}, y_{2}, \eta\right)
$$


Substituting this estimate in the right hand side of (15.86) and neglecting slow (logarithmic) variations of the $g^{-1}$-factor, we can write down the answer, summing up all successive iterations:

$$
\widetilde{D}_{A}\left(\ell_{1}, y_{2}, \eta\right) \approx \frac{C_{A}}{N_{c}} D_{G}\left(\ell_{1}, y_{1}\right) D_{G}\left(\ell_{2}, y_{2}\right) \sum_{k=1}^{\infty} g^{-k}=\frac{N_{c}}{C_{A}} \frac{D_{A}\left(\ell_{1}, y_{1}\right) D_{A}\left(\ell_{2}, y_{2}\right)}{g-1}
$$

Finally, for the double inclusive cross section (5.82) we obtain

$$
\frac{\omega_{1} \omega_{2}}{\sigma} \frac{d^{2} \sigma}{d \omega_{1} d \omega_{2}} \approx \frac{\omega_{1} d \sigma}{\sigma d \omega_{1}} \frac{\omega_{2} d \sigma}{\sigma d \omega_{2}}\left[1+\frac{N_{c}}{3 C_{A}} \frac{1}{1+\frac{4}{3} \sinh ^{2} \frac{\mu_{1}-\mu_{2}}{2}}\right] .
$$

This result can be applyed to the general case of an arbitrary jet ensemble with $C_{A}=\rho$ standing for its total "color charge", e.g., $C_{A}=2 C_{F}$ for $e^{+} e^{-} \rightarrow q \bar{q}$, $c f$. (5.38). According to (5.89) the correlation is maximal for particles with energies of the same order ("equal" energies in the logarithmic scale). 
Problem 5.1 Using the double-differential parton spectrum (5.81) derive inclusive angular distribution of particles.

Answer:

Inclusive angular distribution of particles one gets integrating (5.81) over energies. Invoking power series for the modified Bessel function $I_{0}$

$$
I_{0}(z)=\sum_{n=0}^{\infty} \frac{1}{(n !)^{2}}\left(\frac{z}{2}\right)^{2 n}
$$

one obtains after integrating over $\ell=\ln (1 / x)$

$$
\begin{gathered}
\int_{0}^{Y_{\Theta}} d \ell \frac{\left(\gamma_{0} \sqrt{\left(Y_{\Theta}-\ell\right) \ell}\right)^{2 n}}{(n !)^{2}}=Y\left(\gamma_{0} Y_{\Theta}\right)^{2 n} \frac{1}{(2 n+1) !} \\
\frac{d n}{d \ln \Theta}=\frac{C_{A}}{N_{c}} \cdot \gamma_{0} \cdot \sinh \left(\gamma_{0} Y_{\Theta}\right) .
\end{gathered}
$$

Integral over angles of (5.90) reproduces, in turn, the total particle multiplicity $(c f .(15.50)$ for the gluon jet):

$$
\bar{n}=\frac{C_{A}}{N_{c}} \cdot\left\{\cosh \left(\gamma_{0} Y\right)-1\right\}+1
$$

(The last unity we added here to account for the leading particle originating the jet).

Problem 5.2 Using recurrency relations (5.64), (5.65) derive the second multiplicity moment $f_{2}^{(\rho)}$ and the normalized dispersion of the multiplicity distribution $D^{2} /\langle n\rangle^{2} \equiv f_{2}^{(\rho)}-1$

\section{Answer:}

Keep trying until you reach the value which coincides with the maximum of the two-particle correlation function $\widetilde{D}_{\rho} / D_{\rho}^{2}=N_{c} / 3 C_{A}$, see (15.89). 


\section{Bibliography}

1. A. Bassetto, M. Ciafaloni and G. Marchesini. Phys. Rep., C100:201, 1983.

2. A. Bassetto, M. Ciafaloni, G. Marchesini and A.H. Mueller. Nucl. Phys., B207:189, 1982.

V.S. Fadin. Sov. J. Nucl. Phys., 37:245, 1983.

3. B.I. Ermolayev and V.S. Fadin. JETP. Lett., 33:285, 1981.

A.H. Mueller. Phys. Lett., 104B:161, 1981.

4. B.I. Ermolayev, V.S. Fadin and L.N. Lipatov. Sov. J. Nucl. Phys., 45:508, 1987.

5. V.N. Gribov. Sov. J. Nucl. Phys., 5:280, 1967.

6. R. Kirschner and L.N. Lipatov. Sov. Phys. JETP, 54:266, 1982.

7. S. Catani, M. Ciafaloni and G. Marchesini. Nucl. Phys., B264:558, 1986.

8. K. Konishi, A. Ukawa and G. Veneziano. Nucl. Phys., B157:45, 1979.

9. Yu.L. Dokshitzer, V.S. Fadin and V.A. Khoze. Z. Phys., C15:325, 1982.

10. Yu.L. Dokshitzer, V.S. Fadin and V.A. Khoze. Z. Phys., C18:37, 1983.

11. A. Erdélyi and others. Higher Transcendental Functions, volume 2. McGRAW-HILL, 1953.

12. Z. Koba, H.B. Nielsen, and P. Olesen. Nucl. Phys., B40:317, 1972.

13. A.M. Polyakov. Sov. Phys. JETP, 32:296, 1971; ibid. 33:850, 1971.

14. A. Bassetto, M. Ciafaloni and G. Marchesini. Nucl. Phys., B163: 477, 1980. 


\section{Chapter 6}

\section{Modified Leading Logarithmic Approximation}

\section{Contents}

6.1 Single Logarithmic Corrections to DLA Cascades 188

6.2 MLLA Evolution Equations for Intrajet Cascades 195

6.3 Pattern of Multiple Gluon Bremsstrahlung . . . . 199

6.3.1 The BCM Ansatz and the Dipole Scheme . . . . . 199

6.3 .2 Construction of the Probabilistic Scheme . . . . . 201

$6.3 .3 \quad$ Jet Polarizability and Color Monsters . . . . . . . 213

6.3 .4 Magnitude of Dipole Corrections . . . . . . . . . 218

Bibliograph . . . . . . . . . . . . . 220 
This Chapter is devoted to the description of the PT technique which has been designed in the middle of 1980's for quantitative description of the properties of particles with relatively small momenta $\left(x=k / E_{j e t} \ll 1\right)$, produced in hard interactions. The practical importance of the subject stems simply from the fact that such soft particles, which take away a negligible portion of the jet energy, form, at the same time, the bulk of multiplicity.

We shall follow the logic of the Leading Log Approximation approach to DIS and $e^{+} e^{-}$structure functions in hard momenta region $(x \sim 1)$ described in Chapter 5, which maintains a clear probabilistic picture of the jet development.

\subsection{Single Logarithmic Corrections to DLA Cascades}

As we know from the previous Chapter, strong AO was proved to provide the basis for the probabilistic interpretation of soft gluonic cascades in the Double Logarithmic Approximation :

$$
k_{s} \ll k_{f} \ll k_{g} \quad, \quad \Theta_{s f} \ll \Theta_{f g}
$$

where subscripts denote the cascade genealogy: "grandpa", "father" and "son".

The DLA happens to be too crude however for making reasonable predictions even for asymptotically high energies. On the qualitative level, DLA can be thought to overestimate cascading processes, ignoring completely the energy-momentum balance since the energy of the radiating particle remains 
unchanged (in the soft limit) after a gluon emission. Therefore it seemingly overestimates gluon multiplicities, the characteristic energy of partons that multiplicate most actively (i.e. position of the "hump") etc. Quantitatively, DLA keeps trace of $\sim \sqrt{\alpha}_{s}$ term in the anomalous dimension $\gamma$ disregarding contributions of the order of $\Delta \gamma \sim \alpha_{s}$ which give rise to significant preexponential energy-dependent factors. Thus to make a first step towards the quantitative control over parton generation processes one is forced to take into full account nonleading Single Logarithmic (SL) effects.

Constructing a Probabilistic Scheme with account of both DL and essential SL effects one has to pay for better accuracy of the approximation by a tremendous growth of the number of interference contributions which must be analyzed and interpreted. The interference graphs contain soft gluon lines connecting harder partons of quite different generations. Meanwhile, the very idea of the classical shower picture implies that the structure of elementary parton decays, i.e. the "blocks" for building up the partonic cascade, should depend on just the nearest "forefathers" of a considered parton. Thus the possibility to absorb all essential interference terms into the local probabilistic scheme looks far from being obvious.

The more striking, therefore, is the fact that such a scheme not only exists but appears to be a posteriori a simple almost trivial generalization of the standard LLA scheme described in Chapter 1. That is the reason to refer to this approximation to soft particle physics 12 as the Modified LLA (MLLA).

To Understand and Evaluate subleading corrections to DLA asymptotics it is helpful to invoke the notion of Generating Functional (GF) which has been exploited in Chapter 5 . GF technique is perfectly suited for describ- 
ing intrajet cascades. Its structure can be expressed symbolically as

$$
Z=C\left(\alpha_{s}(t)\right) * \exp \left\{\int^{t} \gamma\left(\alpha_{s}\left(t^{\prime}\right)\right) d t^{\prime}\right\}
$$

This representation incorporates the fact that independent sequential (with respect to the "evolution-time" parameter, $t$ ) elementary processes exponentiate. It exhibits the property of locality inherent to the probabilistic shower picture. Namely, the "time" derivative of (6.2) produces the factor $\gamma\left(\alpha_{s}(t)\right)$ showing that the rate of the "time" change of $Z$ (and, thus, of the particle content of the shower) is determined by the quantity $\gamma(t)$ depending exclusively on the value of the coupling at this "time" scald without any remembrance of prehistory.

Comparing this observation with the notation introduced in Chapter 1 , where we have studied the LLA evolution equations, it is natural to call our symbolic quantities $\gamma$ and $C$, respectively, the Anomalous Dimension and Coefficient Function. Due to AO, the "evolution time" $t$ of (6.2) has to be related to the jet opening angle $d t=d \Theta / \Theta$. This means that all the contributions which are singular in the relative angle between partons should be attributed to the intrajet evolution and must be absorbed into the exponential factor of (6.2). Thus, the exponent of the integrated anomalous dimension $\gamma$ incorporates the Markov chains of sequential angular ordered partonic decays. Meantime, the regular coefficient factor $C$, being free of collinear (or mass) singularities, could be said to describe wide-angle partonic configurations, i.e., multijet contributions to the evolution of the system.

Successive terms of symbolic PT series for $\gamma\left(\alpha_{s}\right)$

$$
\gamma=\sqrt{\alpha}_{s}+\alpha_{s}+\alpha_{s}^{3 / 2}+\alpha_{s}^{2}+\ldots
$$

\footnotetext{
${ }^{1}$ The derivative of $C\left(\alpha_{s}\right)$ produces subleading terms preserving the locality in $t$ also.
} 
correspond to the increasing accuracy in description of elementary partonic decays at parametrically small angles $\Theta_{i j} \ll 1$ and thus of the jet evolution. Keeping trace of subleading corrections to the coefficient function

$$
C=1+\sqrt{\alpha}_{s}+\alpha_{s}+\ldots
$$

one will account for the ensembles of increasing number of such jets with large relative angles $\Theta_{i j} \sim 1$.

An Estimate of $\gamma\left(\alpha_{s}\right) \quad$ comes from a simple DLA Evolution Equation for parton multiplicity depending on the product of the energy and the opening angle of a jet

$$
N(p \Theta) \approx \int^{\Theta} \frac{d \Theta^{\prime}}{\Theta^{\prime}}\left[\int_{0}^{1} \frac{d z}{z} 4 N_{c} \frac{\alpha_{s}}{2 \pi}\right] \cdot N\left(z p \Theta^{\prime}\right)
$$

Comparing with (6.2) one can easily see that the expression in square brackets in (6.5) represents the anomalous dimension. Since both l.h.s. and r.h.s. of (6.5) contain multiplicity factors of the same order of magnitude, the two logarithmic integrations have to compensate $\alpha_{s}$ :

$$
\int d t^{\prime} \int \frac{d z}{z} \alpha_{s} \sim 1
$$

Therefore $\gamma$ can be estimated as $(c f .(5.36))$

$$
\gamma^{D L A}\left(\alpha_{s}\right)=\int \frac{d z}{z} \alpha_{s}=\alpha_{s} \ell \sim \sqrt{\alpha_{s}}
$$


The logarithmic integral over longitudinal gluon momentum ( we denoted by $\ell$ ) contributes effectively as

$$
\int \frac{d z}{z}=\int \frac{d k}{k} \equiv \ell \sim \alpha_{s}^{-1 / 2} .
$$

Integrating $\gamma\left(\alpha_{s}\right)$ estimated by (6.6) in (6.2) one arrives at the characteristic exponent $\exp (c \sqrt{\ln E})$ which describes the rate of the multiplicity growth in DLA, $c f$. (5.53).

The next subleading term from the PT series (6.3) for $\gamma\left(\alpha_{s}\right)$, i.e. $\quad \Delta \gamma \sim$ $\alpha_{s}$, causes significant energy dependence $\exp \left(c_{1} \ln \ln (E / \Lambda)\right) \propto \alpha_{s}(E)^{-c_{1}}$ as well.

To describe it correctly one has to analyze the following subleading effects:

1. Exact $\alpha_{s}($ ?) Prescription i.e. possible influence of the $\ln (1 / z)$ dependence of the running coupling argument in a soft $g$ emission

$$
\Delta \gamma=\int\left(\Delta \alpha_{s}\right) \frac{d z}{z}=\int\left(\alpha_{s}^{2} \ell\right) \frac{d z}{z}=\alpha_{s}^{2} \ell^{2} \sim \alpha_{s},
$$

2. Hard Parton Decays i.e. $g \rightarrow q \bar{q}$ and $q \rightarrow q g, g \rightarrow g g$ splitting with hard momenta $z \sim 1$

$$
\Delta \gamma=\int \alpha_{s} d z \sim \alpha_{s},
$$

3. Exact Angular Integration i.e. the kinematical region of the angles of the same order of magnitude $\Theta_{s f} \sim \Theta_{f g} \sim \Theta_{s g}$ in the $g \rightarrow g g g$ (or $q \rightarrow q g g)$ "double-soft" emission of a gluon pair

$$
\Delta \gamma=\int \alpha_{s}^{2} \frac{d z_{1}}{z_{1}} \frac{d z_{2}}{z_{2}}=\alpha_{s}^{2} \ell^{2} \sim \alpha_{s} .
$$


With account of these effects in the MLLA one gets symbolically

$$
\gamma^{M L L A}\left(\alpha_{s}\right)=\sqrt{\alpha}_{s}+\alpha_{s} .
$$

Two Alternative Approaches had been used to calculate SL effects. The standard renormalization group approach 1 and the probabilistic approach 2 based on the parton shower picture. The main idea of the shower picture is to reorganize the perturbative expansion in such a way that its zero-order approximation is systematic and involves an arbitrary number of produced particles. This zero-order approximation can be achieved through an iteration of basic $A \rightarrow B+C$ parton branchings. In principle, it should be possible to include higher corrections to the basic branching along with higher point branching vertices $A \rightarrow B+C+D \ldots$ in order to improve the accuracy of a calculation. It is important to mention that the choice of an appropriate evolution parameter (i.e. the jet opening angle) makes it possible to incorporate all substantial subleading SL terms without such a complication. As compared to the shower approach, the renormalization group technique, being much better formalized for systematic study of higher order corrections, happens to be less transparent since the branchings are not so visible here.

MLLA Parton Decay Probabilities look as follows:

$$
\begin{aligned}
d w_{A}^{B C} & =\frac{\alpha_{s}\left(k_{\perp}^{2}\right)}{2 \pi} \Phi_{A}^{B C}(z) d z V(\vec{n}) \frac{d \Omega}{8 \pi}, \\
V_{f(g)}^{s}(\vec{n}) & =\frac{a_{s g}+a_{f g}-a_{s f}}{a_{s f} a_{s g}},
\end{aligned}
$$

where subscripts refer to the "soft gluon family" as before. 
Hereafter we denote by $a_{i k}$ the angular factor

$$
a_{i k}=q^{2} \frac{\left(p_{i} \cdot p_{k}\right)}{\left(p_{i} q\right)\left(p_{k} q\right)}=1-\vec{n}_{i} \vec{n}_{k}=1-\cos \Theta_{i k} .
$$

Equation (6.11) takes into full account the SL effects (6.7)-(6.9):

- $k_{\perp}$-prescription solves the problem of running coupling,

- DGLAP nonregularized (sic!) splitting functions $\Phi_{A}^{B C}$ include both soft gluon emission and terms corresponding to a loss of energy logs in $A \rightarrow B+C$ decays,

- the exact angular kernel $V(\vec{n})$ depending on the directions of momenta of partons of three sequential generations replaces the rough strong AO (6.1).

To see that the angular factor (6.12) leads to nothing but the strict $\mathbf{A O}$, the reader is advised to check the nice property of the $V$-kernel:

$$
\left\langle V_{f(g)}^{s}(\vec{n})\right\rangle_{\text {azimuth average }}=\int_{0}^{2 \pi} \frac{d \phi}{2 \pi} V_{f(g)}^{s}(\vec{n})=\frac{2}{a_{s f}} \vartheta\left(a_{f g}-a_{s f}\right),
$$

with $\vartheta$ the step function. This means that the decay probability integrated over the azimuth of "son" around "father" results in the logarithmic $\Theta$ distribution inside the parent cone $\Theta_{s f} \leq \Theta_{f g}$ and vanishes outside.

It is important to emphasize in advance that the " $V$-scheme" (6.11) proves to eliminate from the Evolution Equations both the second and the third loop corrections, that might originate from the local in $t 2$ - and 3 -gluon radiation processes 


$$
\begin{array}{ll}
A \rightarrow A+g^{\prime}+g^{\prime \prime}, & \Delta \gamma=\alpha_{s} ; \\
A \rightarrow A+g^{\prime}+g^{\prime \prime}+g^{\prime \prime \prime}, & \Delta \gamma=\alpha_{s}^{3 / 2}
\end{array}
$$

with $k^{\prime \prime \prime} \ll k^{\prime \prime} \ll k^{\prime} \ll k_{A}$. These processes do not enter the Evolution Equations as new elementary splittings, since the $V$-scheme factorizes them completely into chains of two-parton decays.

The first specific soft contribution arises only in the $4^{\text {th }}$ loop because of subtle interferences between a parent parton and its four offspring gluons with strongly ordered energies and emission angles of the same order. It contributes to the anomalous dimension as $\Delta \gamma \lesssim \alpha_{s}^{4} \ell^{4} \sim \alpha_{s}^{2}$, happens to have $1 / N_{c}^{2}$-suppressed color factor ("color monsters") and could be interpreted physically in terms of the "color polarizability" of a jet (see below). Detailed discussion of these topics can be found in the review 3 .

\subsection{MLLA Evolution Equations for Intrajet Cascades}

When studying angular insensitive characteristics of partonic systems, such as mean multiplicities and multiplicity fluctuations, energy particle spectra and correlations etc., one is allowed to replace the full angular kernel $V(\vec{n})$ in (6.11) by its azimuth averaged analog (6.14). In this case the strict AO makes it possible to construct simple Evolution Equations for GFs.

The system of two coupled equations for functionals $Z_{F}$ and $Z_{G}$ describing parton content of quark and gluon jets with initial momentum $p$ and the 
opening angle $\Theta$ reads $(A, B, C=F, G)$ :

$$
\begin{aligned}
& Z_{A}(p, \Theta ; u(k))=e^{-w_{A}(p \Theta)} u_{A}(k=p)+\frac{1}{2} \sum_{B, C} \int^{\Theta} \frac{d \Theta^{\prime}}{\Theta^{\prime}} \int_{0}^{1} d z e^{-w_{A}(p \Theta)+w_{A}\left(p \Theta^{\prime}\right)} \\
& \times \frac{\alpha_{s}\left(k_{\perp}^{2}\right)}{2 \pi} \Phi_{A}^{B C}(z) Z_{B}\left(z p, \Theta^{\prime} ; u\right) Z_{C}\left((1-z) p, \Theta^{\prime} ; u\right) .
\end{aligned}
$$

The first term in the r.h.s. corresponds to the form factor damped situation when the $A$-jet consists of the parent parton only. The integral term describes the first splitting $A \rightarrow B+C$ with angle $\Theta^{\prime}$ between the products. The exponential factor provides this decay being the first one indeed: it is the probability to emit nothing in the angular interval between $\Theta^{\prime}$ and $\Theta$. The two last factors account for the further evolution of the produced subjets $B$ and $C$ having smaller energies and smaller $\Theta^{\prime}$ as the opening angle.

MLLA Form Factors look as follows $(c f .(1.96))$ :

$$
\begin{aligned}
& w_{F}=\int^{\Theta} \frac{d \Theta^{\prime}}{\Theta^{\prime}} \int_{0}^{1} d z \frac{\alpha_{s}\left(k_{\perp}^{2}\right)}{2 \pi} \Phi_{F}^{F}(z), \quad(F=q, \bar{q}) \\
& w_{G}=\int^{\Theta} \frac{d \Theta^{\prime}}{\Theta^{\prime}} \int_{0}^{1} d z \frac{\alpha_{s}\left(k_{\perp}^{2}\right)}{2 \pi}\left[\frac{1}{2} \Phi_{G}^{G}(z)+n_{f} \Phi_{G}^{F}(z)\right] .
\end{aligned}
$$

Collinear and soft singularities which are present in 6.15] - 6.16b may be regularized by imposing usual for PT restriction on relative transverse momentum of produced partons, which we shall write here in the following form:

$$
k_{\perp} \approx p z(1-z) \Theta^{\prime}>Q_{0} .
$$


The Master Equation which we shall exploit actively for studying properties of QCD jets in the next Chapter can be derived from (6.15) by differentiating the product

$$
Z_{A}(\Theta) e^{w_{A}(p \Theta)}
$$

over $\Theta$. Then, making use of (6.16) one arrives at the system of two coupled differential equations for GFs, which are free from the DL form factors:

$$
\begin{aligned}
\frac{d}{d \ln \Theta} Z_{A}(p, \Theta) & =\frac{1}{2} \sum_{B, C} \int_{0}^{1} d z \Phi_{A}^{B C}(z) \cdot \frac{\alpha_{s}\left(k_{\perp}^{2}\right)}{2 \pi} \\
& \times\left[Z_{B}(z p, \Theta) Z_{C}((1-z) p, \Theta)-Z_{A}(p, \Theta)\right] .
\end{aligned}
$$

Equations (6.18) accumulate information about azimuth averaged jet characteristics in the MLLA and look similar to the GLAP regularized equations for single-inclusive parton distributions (see Chapter 1). Taking the $n^{\text {th }}$ variational derivative of $Z_{A}$ with respect to the probing functions $u\left(k_{i}\right)$ near the "point" $u=0$ one gets the exclusive $n$-parton cross sections. An expansion of $Z_{A}$ at the "point" $u=1$ generates inclusive parton distributions and correlations. In a close analogy with Section 5.4 .2 where GFs for gluon and quark jets have been constructed in the DLA, the MLLA jet functionals have two important properties:

The Initial condition for solving the system (6.18) reads (cf. (5.34)

$$
\left.Z_{A}(p, \Theta ;\{u\})\right|_{p \Theta=Q_{0}}=u_{A}(k=p) .
$$

The $A$-jet with the hardness parameter $p \Theta$ which is set to the boundary value $Q_{0}$ where the PT-evolution starts, consists of the only parent parton $A$. This natural condition is clearly seen from the integral Evolution Equation (6.15) 
where the second term disappears at $p \Theta=Q_{0}$ together with the form factor in the Born term:

$$
w_{A}\left(Q_{0}\right)=0
$$

The Normalization property of GF, known from our DLA experience (cf. (15.37) ), is the second condition

$$
\left.Z_{A}(p, \Theta ;\{u\})\right|_{u(k) \equiv 1}=1
$$

which can be also easily checked. Indeed, putting $Z \equiv 1$ and $u \equiv 1$ in (6.15), one obtains integral equations for quark and gluon form factors

$$
e^{w_{A}(p \Theta)}=1+\frac{1}{2} \sum_{B, C} \int^{\Theta} \frac{d \Theta^{\prime}}{\Theta^{\prime}} \int_{0}^{1} d z \frac{\alpha_{s}\left(k_{\perp}^{2}\right)}{2 \pi} \Phi_{A}^{B C}(z) e^{w_{A}\left(p \Theta^{\prime}\right)}
$$

which lead directly to the MLLA expressions (6.16) for the total parton decay probabilities.

All the properties 2 of a system of jets produced in some hard interaction can be derived (within the MLLA accuracy) by applying variational derivatives to the proper product of GFs. For example, for $e^{+} e^{-}$annihilation to hadrons at energy $W=2 E$, which is dominated (provided no special event selection is imposed) by two-jet configuration, one has

$$
Z_{e^{+} e^{-}}(W ;\{u\})=\left[Z_{F}(E, \Theta=\pi ;\{u\})\right]^{2} .
$$

\footnotetext{
${ }^{2}$ angular correlations excluded !
} 


\subsection{Angular Pattern of Multiple Gluon Bremsstrahlung}

In this Section we intend to provide a reader with a brief description of the analysis of Feynman diagrams for multiple soft gluon production,

$$
e^{+} e^{-} \rightarrow q \bar{q}+g_{1}+g_{2}+\ldots+g_{N},
$$

which has lead to the probabilistic Evolution Equations (6.18) for the intrajet parton cascades, based on the notion of strict AO.

In the LLA for DIS structure functions and $e^{+} e^{-}$inclusive particle production (Chapter (1) we dealt with collinear logs, and substantial partonic fluctuations consisted of partons with arbitrary energies of the same order of magnitude $(x \sim 1)$. Turning to the problem of soft particle production, we were faced in Chapter 5 with the Double Logarithmic regime, when both collinear and infrared logs acted on the same ground. Now, in attempts to improve the DLA description of soft particle multiplication processes, we have to study the "anti-LLA" kinematics, where $N$ gluons are strongly ordered in energies but, in the same time, have arbitrary emission angles. This is the study of the leading infrared singularities.

\subsubsection{The BCM Ansatz and the Dipole Scheme}

An important step was made by Bassetto, Ciafaloni and Marchesini who proposed the general answer for exclusive cross sections of soft multi-gluon production by the color-singlet $g g$ current

$$
" e^{+} e^{-"} \rightarrow g_{+} g_{-}+g_{1}+g_{2}+\ldots+g_{N} .
$$


The corresponding nice expression was suggested in Ref. [4:

$$
\begin{array}{ll}
\frac{1}{\sigma} d \sigma^{(N)}= & \left(\frac{\alpha_{s} N_{c}}{\pi}\right)^{N} \prod_{i=1}^{N} \frac{d \Omega_{i}}{4 \pi} \frac{d \omega_{i}}{\omega_{i}} \cdot W\left(\left\{\vec{n}_{i}\right\}\right), \\
\text { with } & W\left(\left\{\vec{n}_{i}\right\}\right)=a_{+-}^{2} \sum_{\text {perm }}\left(a_{i_{1} i_{2}} a_{i_{2} i_{3}} \ldots a_{i_{N+2} i_{1}}\right)^{-1}
\end{array}
$$

where summations runs over all possible permutations of $N+2$ indices $i_{k}=$ $+,-, 1,2 \ldots N$ yielding inequivalent factors. The "relative angles" functions $a_{i k}$ were defined in (6.13). As an example, the angular pattern for the two soft gluon production reads

$$
W\left(\vec{n}_{1}, \vec{n}_{2}\right)=a_{+-}^{2}\left[\frac{1}{a_{+1} a_{1-} a_{-2} a_{2+}}+\frac{1}{a_{+-} a_{-1} a_{12} a_{2+}}+\frac{1}{a_{+-} a_{-2} a_{21} a_{1+}}\right] .
$$

Equation (6.24) had been checked for $N \leq 3$. In higher orders, however, this ansatz breaks down 2, representing the true result only in the large- $N_{c}$ approximation 5 .

Meanwhile, within this accuracy, the cross sections (6.24) can be used to formulate the picture of gluonic cascades, accounting for major coherence effects. To illustrate the idea of corresponding Monte Carlo algorithm (the so called "Lund-dipole-scheme", see Ref. [6]) let us present the cross section for $N=2$ in explicitly Lorentz-invariant form as

$$
\begin{aligned}
\frac{1}{\sigma} d \sigma^{(2)} & =\left(\frac{\alpha_{s} N_{c}}{\pi}\right)^{2} \prod_{i=1}^{2} \frac{d^{3} k_{i}}{\pi \omega_{i}} \cdot\left[\frac{\left(p_{+} p_{-}\right)}{2\left(p_{+} k_{1}\right)\left(k_{1} p_{-}\right)} \frac{\left(p_{+} p_{-}\right)}{2\left(p_{+} k_{2}\right)\left(k_{2} p_{-}\right)}\right. \\
& \left.+\frac{\left(p_{+} p_{-}\right)}{4\left(p_{-} k_{1}\right)\left(k_{1} k_{2}\right)\left(k_{2} p_{+}\right)}+\frac{\left(p_{+} p_{-}\right)}{4\left(p_{-} k_{2}\right)\left(k_{1} k_{2}\right)\left(k_{1} p_{+}\right)}\right] .
\end{aligned}
$$


The first term in square brackets is the easiest one to interpret. It is the product of the two bremsstrahlung factors for independent gluon radiation by the $(+-)$ current:

$$
\frac{1}{k_{\perp 1}^{2}} \frac{1}{k_{\perp 2}^{2}} ; \quad \frac{2\left(k p_{+}\right)\left(k p_{-}\right)}{\left(p_{+} p_{-}\right)}=\omega^{2}(1-\cos \Theta)(1+\cos \Theta)=k_{\perp}^{2},
$$

where we have taken into account that $\vec{n}_{+}=-\vec{n}_{-}$in the c.m.s. of the hard emitters, $k_{\perp}$ being the transverse component of the gluon momentum $\vec{k}$ with respect to this axis. Two other terms of (6.26) we can rearrange as follows:

$$
\begin{array}{r}
\frac{\left(p_{+} p_{-}\right)}{2\left(k_{1} p_{+}\right)\left(k_{1} p_{-}\right)}\left[\frac{\left(k_{1} p_{+}\right)}{2\left(k_{2} k_{1}\right)\left(k_{2} p_{+}\right)}+\frac{\left(k_{1} p_{-}\right)}{2\left(k_{2} k_{1}\right)\left(k_{2} p_{-}\right)}\right]= \\
\left(\frac{1}{k_{\perp 1}^{2}}\right)_{(+\rightarrow}\left[\left(\frac{1}{k_{\perp 2}^{2}}\right)_{(1+)}+\left(\frac{1}{k_{\perp 2}^{2}}\right)_{(1 \rightarrow)}\right] .
\end{array}
$$

Now we can treat this result as an independent emission of $g_{1}$, followed by the radiation of softer $g_{2}$ by two newly formed color dipoles (1+) and (1-). Bremsstrahlung factors are once again the inverse squared transverse momentum of $g_{2}$ but evaluated in the c.m.s. frames of corresponding emitters. The $N=2$ system will act as an ensemble of $(N+2)(N+1) / 2=6$ dipoles with respect to subsequent radiation of $g_{3}$ etc. We will come back to the dipole picture later in Chapter 9

\subsubsection{Construction of the Probabilistic Scheme}

Placing no restrictions upon the angular pattern of multi-gluon ensembles, one gets in the $N^{t h}$-order the whole series of subleading corrections to the DLA, which we can display symbolically as:

$$
\sigma^{(N)}=\alpha_{s}^{N} \ell^{N}\left(t^{N}+t^{N-1}+\ldots+t+\text { const }\right) .
$$


where $\ell$ and $t$ denote the energy and angle logs as before.

How to deal with this structure? Increasing $N$ order by order, we shall analyze the leading infrared contributions to the Anomalous Dimension (6.3), describing elementary parton decays responsible for the internal structure of a jet according to (6.2). As we shall show, by an appropriate choice of the evolution parameter, the PT-series (6.29) corresponding to a single jet development can be written as

$$
\begin{aligned}
\sum_{N} \sigma^{(N)} & =\exp \left\{\int^{t} \gamma\left(\alpha_{s}\left(t^{\prime}\right)\right) d t^{\prime}\right\} \\
\text { with } \quad \gamma\left(\alpha_{s}\right) & =\alpha_{s} \ell+\left(\alpha_{s} \ell\right)^{4}+\ldots \approx \sqrt{\alpha_{s}}+\alpha_{s}^{2}+\ldots
\end{aligned}
$$

To be more precise, this means that essentially all subleading angular logs are embodied in simple iterations of the elementary one-gluon emission process together with the leading DLogs $\left(\alpha_{s} \ell\right.$ term of (6.30)). (As we know from our DLA experience, such iterations correspond to the exponential form of the GF of a jet.)

Extra term $\left(\alpha_{s} \ell\right)^{4}$ in the Anomalous Dimension (6.30) appears in the $4^{\text {th }}$ loop. In the language of the MLLA Evolution Equation, it induces a new contribution to the r.h.s. of (6.15), in addition to the product of two Generating Functionals $Z_{B} \cdot Z_{C}$, which the product of five $Z$-functionals.

\section{Soft amplitudes and the gauge choice}

The starting point is the construction of multi-gluon amplitudes. In the region of strongly ordered gluon energies with which we are concerned, this can be done by successive application of the factorization property (5.17) which we 
have seen in operation in the previous Chapter:

$$
M^{(N)}=\sum_{i} \frac{\left(e_{N} \cdot k_{i}\right)}{\left(k_{N} \cdot k_{i}\right)} g_{s} T_{i} \cdot M^{(N-1)}, \quad i=+,-, 1,2 \ldots(N-1) .
$$

Here $T_{i}$ is an appropriate color generator for radiation of the softest gluon $g_{N}$ off the $i^{\text {th }}$ particle of the parton system generated in the previous order of PT. The square of this amplitude determines the $N$-particle exclusive cross section (6.23).

In the DLA the angular pattern function $W^{(N)}\left(\left\{\vec{n}_{i}\right\}\right)$ could be interpreted by means of probabilistic cascade with the strong AO $\left(\Theta_{i+1} \ll \Theta_{i}\right)$. Now we are going to show, how by replacing the strong ordering by the strict $\mathrm{AO}$ $\left(\Theta_{i+1} \leq \Theta_{i}\right)$ one gets the subleading $\Theta$-logs of the expansion (6.29) accounted for.

The amplitude generated by the recurrence relation (6.31) is, clearly, gauge invariant. It proves to be convenient to choose the physical planar gauge (5.4) with the gauge vector $c_{\mu}$ connected to the c.m.s. of the $q \bar{q}$ pair, which we exploited in the previous Chapter studying the DLA parton cascades. Let us recall, that such a choice suppresses the interference graphs connecting $(+)$ and $(-)$ lines and thus reduces the number of Feynman diagrams contributing to $W^{(N)}\left(\left\{\vec{n}_{i}\right\}\right)$.

The number of topologically nonequivalent Feynman diagrams for $W^{(N)}\left(\left\{\vec{n}_{i}\right\}\right)$ can be estimated as

$$
\ell_{N} \geq \frac{1}{2} \prod_{m=1}^{N} \frac{m(m+3)}{2}=1,5,45, \sim 700, \ldots, \quad \text { for } \quad N=1,2,3,4, \ldots .
$$

In the planar gauge the gluon " $i$ " connecting two harder partons " $\ell$ " and " $m$ " 
in the Feynman diagrams for $M M^{*}$ introduces the factor

$$
I_{\ell m}^{i}=\left(k_{i} n\right)^{2} \frac{k_{\ell \mu} d_{\mu \nu}\left(k_{i}\right) k_{m \nu}}{\left(k_{\ell} k_{i}\right)\left(k_{m} k_{i}\right)}=\frac{a_{i \ell}+a_{i m}-a_{\ell m}}{a_{i \ell} a_{i m}}
$$

for $\ell \neq m$ ("interference" terms), and

$$
H_{\ell}^{i} \equiv I_{\ell \ell}^{i}=\frac{\left(k_{i} n\right)^{2}}{\left(k_{\ell} k_{i}\right)^{2}}\left(k_{\ell \mu} d_{\mu \nu}\left(k_{i}\right) k_{\ell \nu}\right)=\frac{2}{a_{i \ell}}
$$

for $\ell=m$ ("self-energy" terms). With these elements we start to construct a desired probabilistic scheme.

\section{Conditional probability and the "dipole" remainder}

$\underline{N=1}$ case

$$
W^{(1)}=C_{F} \cdot\left(H_{+}^{1}+H_{-}^{1}\right)
$$

can be naturally interpreted as the sum of probabilities of independent emission of $g_{1}$ by the $q$ and $\bar{q}$. 
$\underline{N=2}$ Let us list the contributions related to $g_{1}$-radiation off the $q$ :
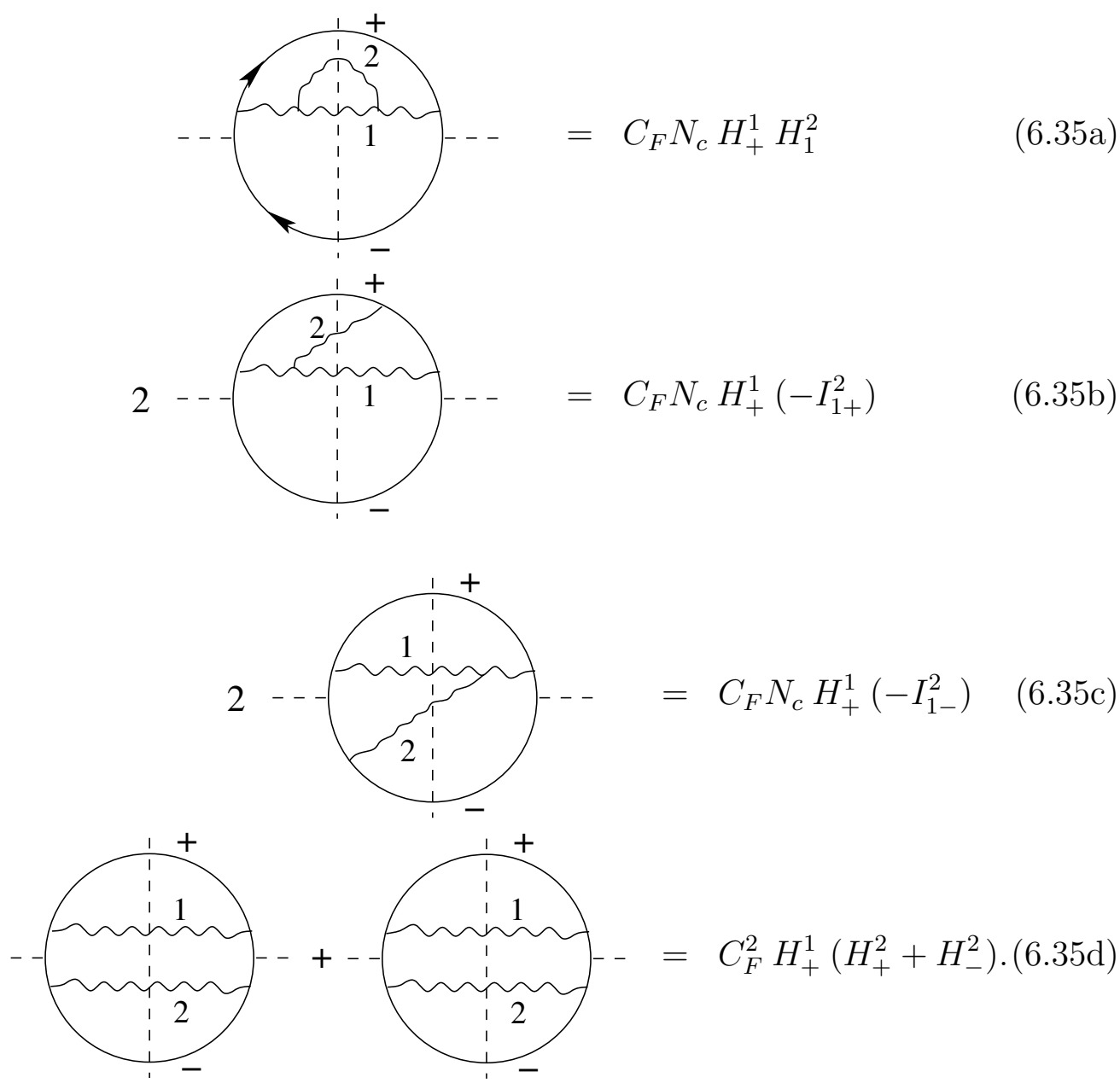

The other part (emission of $g_{1}$ off the $\bar{q}$ ) can be obtained via symmetrization $(+) \leftrightarrow(-)$. The graphs (6.35) have essentially different angular behavior. 
The item $(a)$ is singular both at $a_{21} \rightarrow 0$ and $a_{1+} \rightarrow 0$.

When $g_{2}$ is emitted at large angles, $a_{2+} \approx a_{21} \gg a_{1+}$, then $I_{1+}^{2} \approx H_{1}^{2}$ (see (6.32) $)$ and items $(a)$ and $(b)$ cancel each other, reproducing thus the strong $\mathrm{AO}$ of the DLA.

Contrary to $(a)$ and $(b)$, the item $(c)$ has no singularity at all. Strictly speaking, the pole in $I_{1-}^{2}$ exists when, e.g., $a_{21} \rightarrow 0$ :

$$
I_{1-}^{2} \propto \sin \Theta_{21} / a_{21} \sim 1 / \Theta_{21} .
$$

But it is reasonable to think of this behavior as non-singular, because such a pole gives no angular log in the integral cross section. Cancellation of the singularity of the $H_{+}^{1}$-factor in (6.35c) at $a_{1+} \rightarrow 0$ can be made transparent by rewriting $-I_{1-}^{2}=D_{-[+1]}^{2}$, where we introduced the difference of two interference contributions

$$
D_{l[m n]}^{i} \equiv I_{l m}^{i}-I_{l n}^{i}
$$

and used the fact that $I_{+-}^{2}=0$ due to the property of the gauge we have chosen.

The structures of this type, (6.36), we shall rather often meet in what follows. It seems natural to call them the "angular dipoles".

Such non-singular dependence on the angles between any pair of particles $(i, j)$ we call hereafter the "friability" of a given contribution.

Less evident is another property of the item $(c)$ - its global integrability over directions of all the gluons involved. We shall call this property the "ideality" of a contribution. It means that the term under consideration contains not a single angular log and describes, therefore, configurations with all the relative angles being large: $\Theta_{i j} \sim 1$.

With help of the formula

$$
\int \frac{d \Omega_{i}}{4 \pi} I_{l m}^{i}=\ln \frac{2}{a_{l m}}
$$


one obtains for the item $(c)$

$$
\int \frac{d \Omega_{2}}{4 \pi} \int \frac{d \Omega_{1}}{4 \pi} H_{+}^{1} D_{-[+1]}^{2}=\int_{0}^{1} \frac{d x}{1-x} \ln x \equiv-\zeta(2)=-\frac{\pi^{2}}{6} .
$$

The ideality of the item (6.35c) is a reason to consider this term as the "remainder" $R$, excluding it from the definition of conditional probability $V$ which we then define as

$$
V_{1(+)}^{2}=H_{1}^{2}-I_{1+}^{2}=\frac{a_{2+}+a_{1+}-a_{21}}{a_{21} a_{2+}} .
$$

Finally, $W^{(2)}$ gets the following representation:

$$
W^{(2)}=P^{(2)}+R^{(2)},
$$

where $P^{(2)}$ corresponds to the probabilistic scheme

$$
P^{(2)}=C_{F}\left(H_{+}^{1}+H_{-}^{1}\right) \cdot C_{F}\left(H_{+}^{2}+H_{-}^{2}\right)+C_{F} H_{+}^{1} \cdot N_{c} V_{1(+)}^{2}+C_{F} H_{-}^{1} \cdot N_{c} V_{1(-)}^{2}
$$

and the remainder $R^{(2)}$ reads

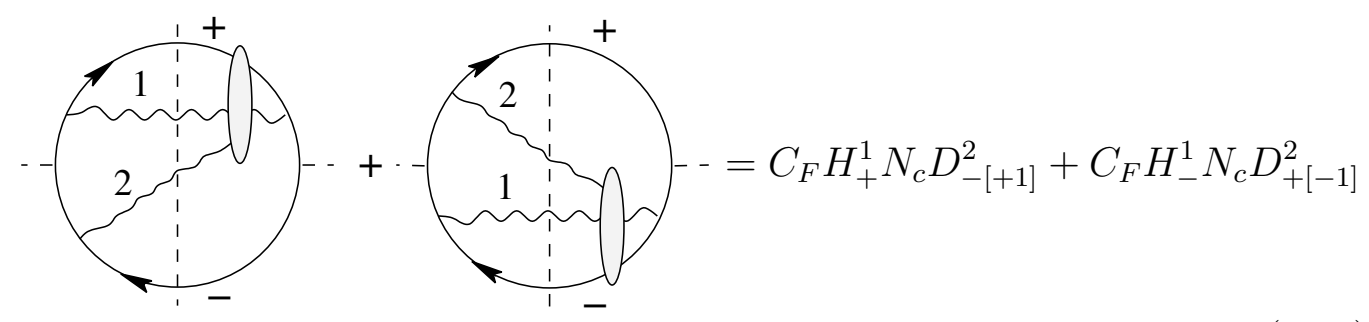


Decomposition (6.40) with the "ideal" remainder $R$ means that in the second order both leading and subleading angular logs in

$$
\sigma^{(2)}=\alpha_{s}^{2} \ell^{2} \cdot\left(t^{2}+t+\text { const }\right)
$$

have been embodied in the iterative scheme with the $V$-functions as the angular kernels. Let us remark for completeness, that the $H$-factors, we put for the emission off the quark lines, could be easily rewritten in terms of $V$-kernels as well:

$$
H_{+}^{1}+H_{-}^{1}=V_{+(-)}^{1}+V_{-(+)}^{1}
$$

Thus, no next-to-leading specific "soft" correction to the Anomalous Dimension $\gamma\left(\alpha_{s}\right)$ (6.3), which might be as large as

$$
\Delta \gamma \sim \alpha_{s}^{2} \ell^{2} \sim \alpha_{s},
$$

arisen from the second loop.

The role of the remainder $R^{(2)}$ consists of providing the first "soft" correction to the Coefficient Function $C\left(\alpha_{s}\right)$ (6.4). As we shall see a little later on, this term contributes to inclusive characteristics of a hard process as $\Delta C=O\left(\alpha_{s}\right)$.

Hereafter we take the conditional probability $V_{f(g)}^{s}$ defined by (6.39) as the basic element for constructing parton cascades in higher orders. As it was mentioned above, with this choice we'll be able to substitute effectively the strong AO by the strict one (see Problem 6.1).

$\underline{N=3} \quad$ Now, with the help of the $V$-kernels let us write down the main probabilistic part $P^{(3)}$ accounting for all possible independent and cascade radiation sequences:

$$
P^{(3)}=P_{0}^{(3)}+P_{1}^{(3)}+P_{2}^{(3)},
$$




$$
\begin{array}{rrr}
P_{0}^{(3)}= & C_{F} H_{+}^{1}\left[C_{F}\left(H_{+}^{2}+H_{-}^{2}\right) \cdot C_{F}\left(H_{+}^{3}+H_{-}^{3}\right)\right], \\
P_{1}^{(3)}= & C_{F} H_{+}^{1}\left[C_{F}\left(H_{+}^{2}+H_{-}^{2}\right) \cdot N_{c} V_{1(+)}^{3}+C_{F} H_{+}^{2} \cdot N_{c} V_{2(+)}^{3}\right. \\
& \left.\quad+C_{F} H_{-}^{2} \cdot N_{c} V_{2(-)}^{3}+N_{c} V_{1(+)}^{2} \cdot C_{F}\left(H_{+}^{3}+H_{-}^{3}\right)\right], \\
P_{2}^{(3)}= & C_{F} H_{+}^{1}\left[N_{c} V_{1(+)}^{2} \cdot N_{c} V_{1(+)}^{3}+N_{c} V_{1(+)}^{2} \cdot N_{c} V_{2(1)}^{3}\right] .
\end{array}
$$

Symmetrization $(+) \leftrightarrow(-)$ is implied.

Each of $P_{i}^{(3)}$ corresponds to a certain sequence of gluon emissions:

$P_{0}^{(3)}$ is independent radiation of $g_{1}, g_{2}, g_{3}$ by $q_{+}$and $\bar{q}_{-}$;

$P_{1}^{(3)}$ describes three similar processes with two independently emited gluons, one of which produces one softer parton starting thus to form its own subjet $\left(H_{+,-}^{i} \equiv\right.$ $\left.H_{+}^{i}+H_{-}^{i}\right)$,
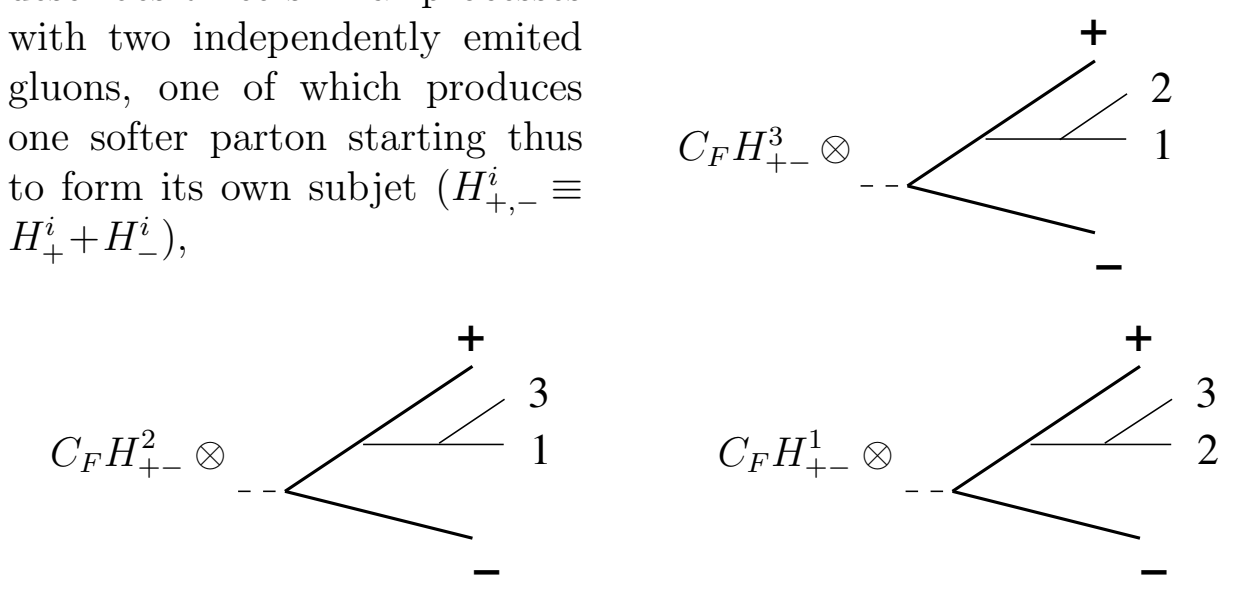
$P_{2}^{(3)}$ consists of one subjet $g_{1}$ formed by

1. emissions of $g_{2}$ and $g_{3}$, restricted by the direction of their common grandpa $q_{+}$

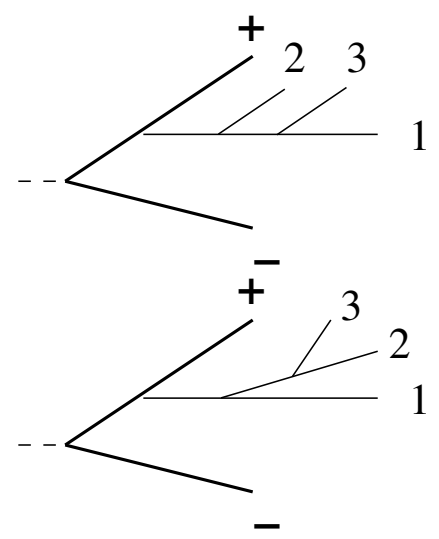

2. the 3-step cascade with successively decreasing opening angles $1 \rightarrow 2 \rightarrow 3$.

The remainder $R^{(3)}$ contains contributions of the two different types:

$$
R^{(3)}=R_{\alpha}^{(3)}+R_{\beta}^{(3)} .
$$

Here $R_{\alpha}^{(3)}=\left(R^{(2)}\right)^{\prime}$ represents the cascade development of the partons involved in $R^{(2)}$; the new irreducible interference structures $R_{\beta}^{(3)}$ are listed 
below:
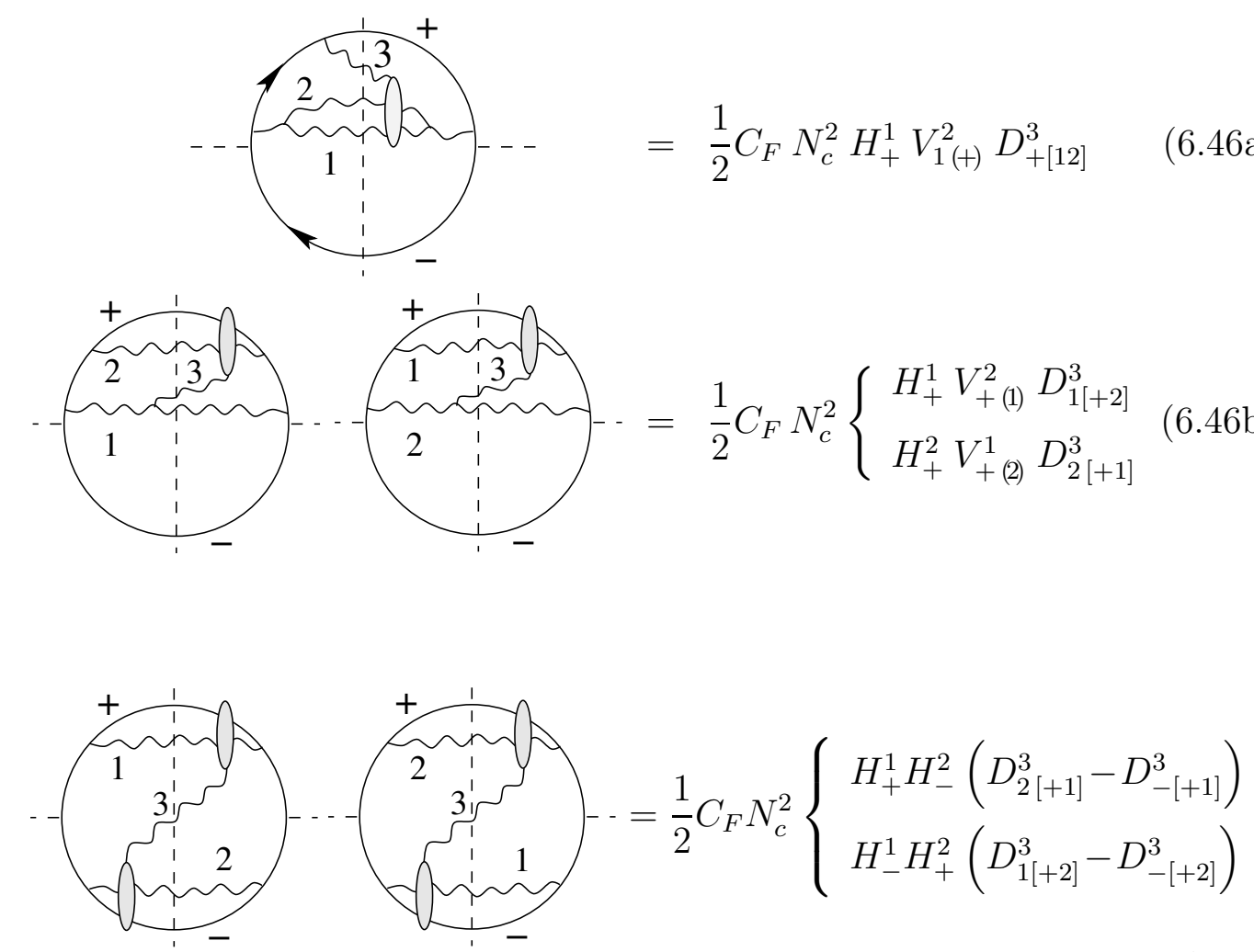


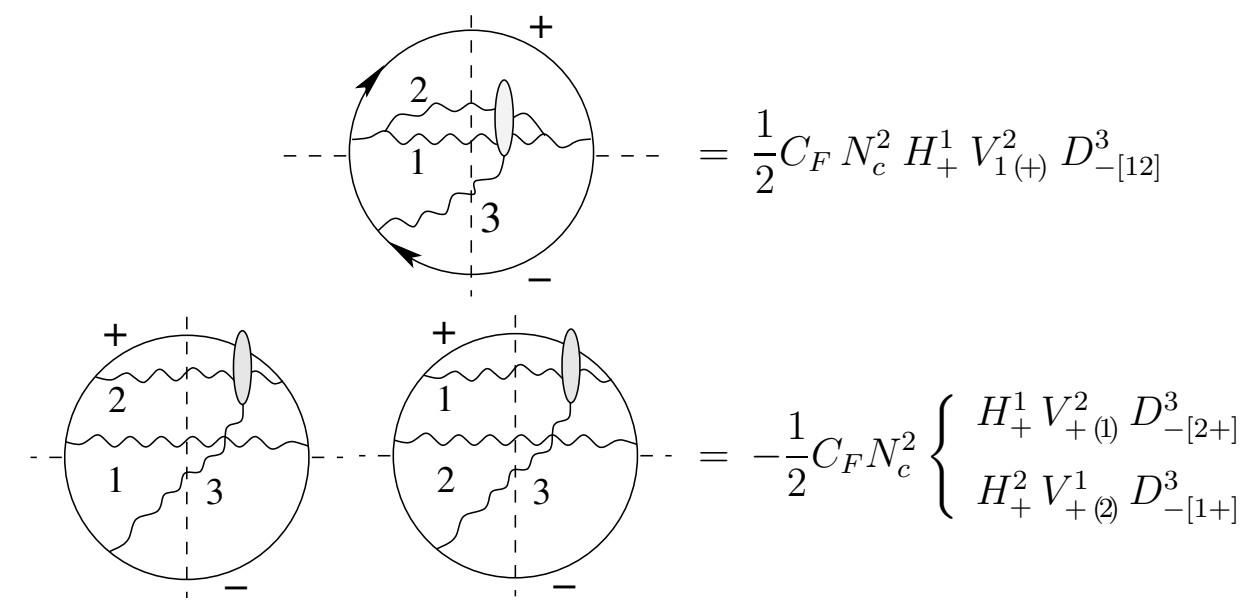

This remainder, similar to the $N=2$ case, proves to have both the properties of friability and ideality.

Let us summarize the result for

$$
W^{(3)}\left(\left\{\vec{n}_{i}\right\}\right)=P^{(3)}+\left(R^{(2)}\right)^{\prime}+R_{\beta}^{(3)} .
$$

The set of Feynman graphs have been split into

$P^{(3)}$ - the probabilistic part, describing independent and cascade emissions of $g_{1}, g_{2}$ and $g_{3}$ (the $V$-scheme);

$\left(R^{(2)}\right)^{\prime}$ - the first expansion term of the product of evolution exponents $Z_{F}^{2} Z_{G^{\prime}} Z_{G^{\prime \prime}}$ describing "4-jet events" with two soft gluon "jets" produced at large angles with respect to the $q \bar{q}$-pair ("renormalization" of the remainder of the previous order); 
$R_{\beta}^{(3)}$ - the new irreducible dipole structure playing the role of the Born term for "5-jet events" $Z_{F}^{2} Z_{G^{\prime}} Z_{G^{\prime \prime}} Z_{G^{\prime \prime \prime}}$ (3 additional soft gluons at large angles).

Now we can repeat the resumé made at the end of the previous paragraph. Namely, the decomposition (6.49) with the "ideal" remainder $R$ shows that in the third order all angular logs in

$$
\sigma^{(3)}=\alpha_{s}^{3} \ell^{3} \cdot\left(t^{3}+t^{2}+t+\text { const }\right)
$$

have been fully accounted by our iterative scheme. Consequently, no next-tonext-to-leading specific "soft" correction to the Anomalous Dimension $\gamma\left(\alpha_{s}\right)$ (6.3) arose, which might be of the order of

$$
\Delta \gamma \sim \alpha_{s}^{3} \ell^{3} \sim\left(\alpha_{s}\right)^{3 / 2}
$$

The remainder $R^{(3)}$ provides the next "soft" correction to the Coefficient Function $\Delta C \sim\left(\alpha_{s}\right)^{3 / 2}$.

\subsubsection{Jet Polarizability and Color Monsters}

The $V$-scheme has been checked for $N=4$ as well 2 2 . It is straightforward to write down all the contributions to $P^{(4)}$. To do that, one has to list all possible two-parton decay sequences and ascribe to them corresponding probabilities according to the $V$-scheme (cf. (6.44) for $N=3$ ).

After $P^{(4)}$ has been singled out, one is left with a cumbersome series of functions depending on the momentum directions of four gluons. The next step consists of separating out the singular pieces which are divergent when one of the relative angles vanishes. It can be easily seen, that such terms 
appear only from collinear emissions from the partons that were involved in building up the remainders of previous PT-orders:

$$
R_{\alpha}^{(4)}=\left(R^{(2)}\right)^{\prime \prime}+\left(R_{\beta}^{(3)}\right)^{\prime} .
$$

Then comes the time to check the character of the remaining irreducible $R_{\beta}^{(4)}$ contribution. It clearly satisfies the property of friability (all one-particle singularities were removed), and the question is about its ideality, i.e. whether it is completely integrable over angles. This was the case, as we have seen above, for $N=3$.

Now, however, the situation changes: $R_{\beta}^{(4)}$ turns out to be non-ideal owing to specific interference between the quark and four gluons with small relative angles of the same order

$$
\Theta_{+i} \sim \Theta_{i k} \sim \Theta \ll 1 \quad i, k=1,2,3,4(i \neq k) .
$$

It is important to notice, that these peculiar contributions, which spoiled the ideality for $N=4$, happen to have nontrivial color factor $C_{F} N_{c}$. Such factor originates from non-planar graphs having color topology of a crossed gluonic square. Examples of the monster graphs are shown in Fig. 6.1.

To make it clear, that $C_{F} N_{c}$ did not come from an interplay of four color charges, e.g., as $C_{F} N_{c}^{2}\left(N_{c}-2 C_{F}\right)$, it is helpful to replace the outer quark pair by hard gluons, to arrive at the same conclusion:

$$
\left\{R_{\beta}^{(4)}\right\}^{\text {non-ideal }} \propto C_{A} \cdot N_{c}
$$

for partons of an arbitrary type $A$ (arbitrary representation of the $S U\left(N_{c}\right)$ ) forming the original (+-) loop. Thus, having radiated four extra gluons, we are left with only the second power of color charges, which clearly breaks 

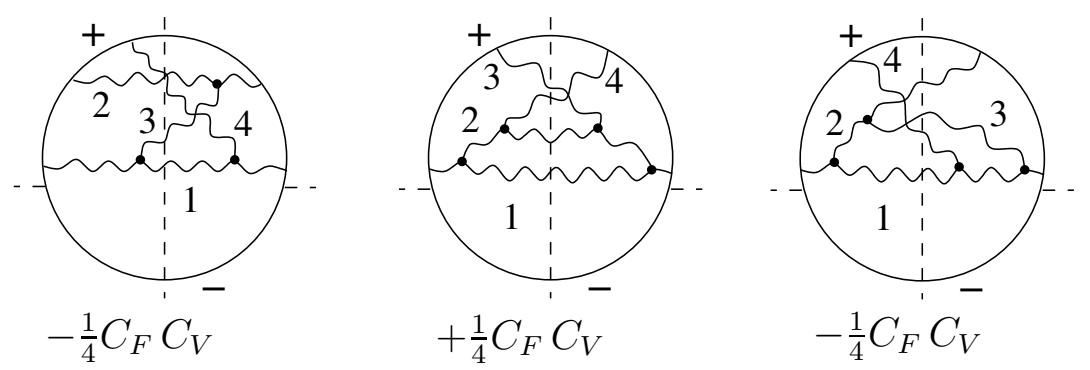

$-\frac{1}{4} C_{F} C_{V}$

Figure 6.1: Examples of the monster graphs with corresponding color factors.

down the naive expectations based on the picture of independent successive gluon emissions.

That is the reason, we call these contributions the "color monsters". The monsters have supplied us with an extra elementary parton branching process, contributing to the Anomalous Dimension (6.30), which corresponds to radiation by the quark (or by the parton $A$, in general) of an "unresolvable", compact in the angular space (according to (6.50) ), group of soft gluons.

Obviously, the total monster contribution to the cross section is gauge invariant since it has a unique color structure. Therefore one can select, once again, the most convenient gauge to analyze it.

The axial gauge $p_{-\mu} A_{\mu}^{a}=0$ where there are only 15 monster diagrams (much less than in the planar gauge) proves to be suitable for that.

The full monster contribution can be represented in a compact way with help of the two following graphs: 


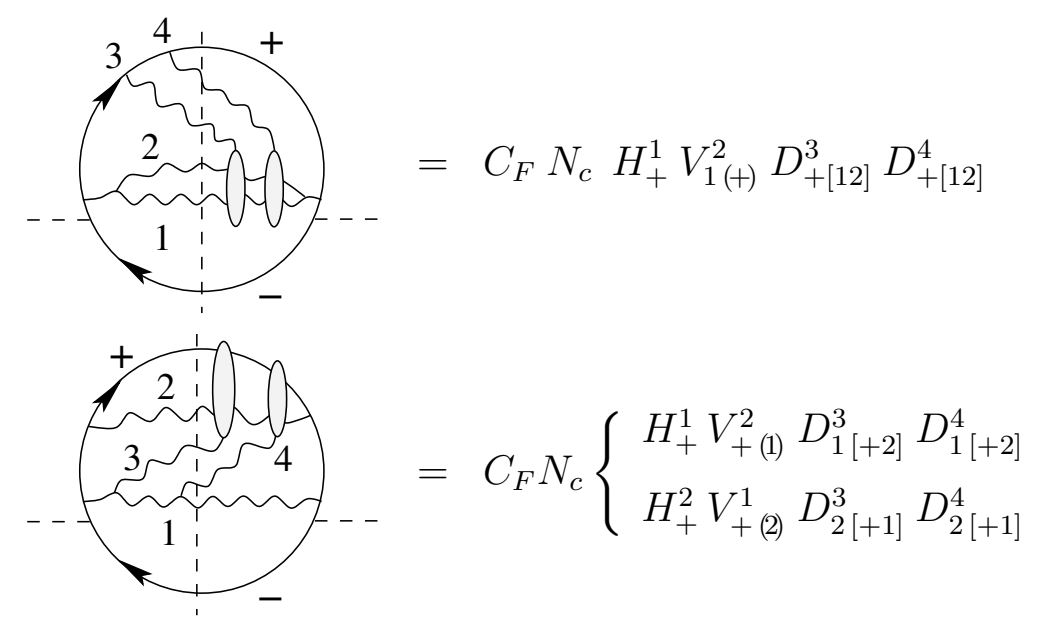

It is implied, that the expressions for conditional probabilities $V$ and dipoles $D$ in (6.51) are written in the axial gauge (see Problem 6.2). To single out the monster contribution to $\gamma$ we restrict ourselves to small gluon angles (6.50) with respect to the $(+)$ direction. Then expressions for $V$ and $D$ coincide (up to negligible powers of $\Theta$ ) with those in the planar gauge, we are familiar with. Then taking the integrals over directions $\vec{n}_{3}$ and $\vec{n}_{4}$ in (6.51a)

$$
\int \frac{d \Omega_{3}}{4 \pi} D_{+[12]}^{3}=\ln \frac{a_{1+}}{a_{2+}},
$$

for the divergent piece of the monster contribution we obtain:

$$
\frac{d \Omega_{1}}{4 \pi} H_{+}^{1} \int \frac{d \Omega_{2}}{4 \pi} V_{1(+)}^{2} \ln ^{2} \frac{a_{1+}}{a_{2+}}=\frac{d a_{1+}}{a_{1+}}\left[\int_{0}^{1} \frac{d x}{1-x} \ln ^{2} x+\int_{a_{1+} / 2}^{1} \frac{d x}{1-x} \ln ^{2} \frac{1}{x}\right]
$$




$$
\approx 4 \zeta(3) \cdot \frac{d a_{1+}}{a_{1+}} .
$$

Two monsters of (6.51b), differing by the energy order of horizontal gluon lines, give the same contribution each.

Now it becomes transparent, what has happened when we added the fourth soft gluon. Graphs of (6.51), but with one dipole instead of two, we have had already at $N=3$, see (6.46). The reason, why those remainder contributions were "ideal", is clear from (6.53): without having squared the dipole logarithms, the two terms in the integrand canceled at $a_{1+}=0$ thus preventing the divergency of the last angular integration.

It seems helpful to invoke the following physical analogy. A "dipole" correction looks like an interaction between the color charge of one jet with the color dipole moment of the other one. When momenta of the partons $m$ and $n$ become parallel, the "dipole" structure $D_{l[m n]}^{i}$ defined by (6.36) vanishes, as an ordinary dipole moment does when opposite charges come close to each other.

Following this physical analogy all the terms of the remainders $R_{\beta}^{(2)}$ and $R_{\beta}^{(3)}$ could be looked upon as various interactions between the color charge of one jet with the color dipole moment of the other one (only except the term (6.47) which looks like a dipole-dipole interaction). Integrating over angles we get vanishing average color dipole momenta $\langle\vec{d}\rangle=0$ which ensures the ideality of remainders.

In terms of our analogy the monsters (6.51) differ substantially: each of them contains a double interaction of one parton with color dipole of a twoparton subjet. But now the square of the dipole moment has a finite average value $\langle\vec{d} \cdot \vec{d}\rangle \neq 0$ because the second interaction, mediated by $g_{4}$, feels a color moment induced by the first one $\left(g_{3}\right)$. Thus it is "color polarizability" of a 
jet which gives rise to monster contributions to the Anomalous Dimension.

\subsubsection{Magnitude of Dipole Corrections}

Let us take the mean multiplicity of $e^{+} e^{-}$annihilation as a testing ground for getting estimate of magnitude of the first dipole correction (6.42). The contribution arises when the registered particle comes from the softest of the dipole jets $\left(g_{2}\right)$; other cuts are to be canceled by corresponding virtual corrections.

After integrating over the directions of $g_{1}$ and $g_{2}$ (see (6.38)) and taking into account that the jet 2 has large opening angle $\Theta_{2} \sim 1$ one gets

$$
\Delta N_{e^{+} e^{-}}(E=W / 2)=2 C_{F} N_{c}\left(-\frac{\pi^{2}}{6}\right) \int^{E} \frac{d k_{1}}{k_{1}} \frac{\alpha_{s}}{2 \pi} \int^{k_{1}} \frac{d k_{2}}{k_{2}} \frac{\alpha_{s}}{2 \pi} N_{G}\left(k_{2}\right),
$$

where $N_{G}\left(k_{2}\right)$ denotes the multiplicity originated from $g_{2}$. Estimating the energy integrals as

$$
\int^{k} \frac{d k^{\prime}}{k^{\prime}} N_{G}\left(k^{\prime}\right) \approx \frac{1}{\gamma_{0}} \cdot N_{G}(k)
$$

we obtain

$$
\frac{\Delta N_{e^{+} e^{-}}}{N_{e^{+} e^{-}}}=-N_{c}^{2} \frac{\pi^{2}}{6} \frac{\left(\alpha_{s} / 2 \pi\right)^{2}}{\gamma_{0}^{2}}=-\frac{N_{c} \alpha_{s}(E)}{2 \pi} \frac{\pi^{2}}{24} .
$$

Equation (6.55) displays the relative smallness of the dipole contribution. Since it proves to be the next-to-next-to-leading correction of the expansion (6.4), the first subleading term in the Coefficient Function, i.e. that of the order of $\sqrt{\alpha_{s}}$ is under the full control of the MLLA Equations (6.18). 
Problem 6.1 Check the main property 6.14) of the conditional probability function $V_{f(g)}^{s}$.

\section{Solution:}

After averaging over the azimuthal angle of $\vec{n}_{s}$ with respect to $\vec{n}_{f}$

$$
\begin{aligned}
\int \frac{d \phi_{s(f)}}{2 \pi} \frac{1}{a_{s g}} & =\frac{1}{\left|a_{f g}-a_{s g}\right|} \\
\int \frac{d \phi_{s(f)}}{2 \pi} V_{f(g)}^{s} & =\frac{1}{a_{s f}}\left(1+\frac{a_{f g}-a_{s f}}{\left|a_{f g}-a_{s f}\right|}\right)
\end{aligned}
$$

one gets immediately:

$$
\left\langle V_{f(g)}^{s}\right\rangle=\frac{2}{a_{s f}} \cdot \vartheta\left(\Theta_{f g}-\Theta_{s f}\right),
$$

which means that the MLLA gluon emission probability coincides with the DLA one inside the bremsstrahlung cone and vanishes outside it.

Problem 6.2 Derive angular functions corresponding to conditional probabilities $V$ and dipoles $D$ in the axial gauge $p_{-\mu} A_{\mu}^{a}=0$.

\section{Answer:}

$$
\begin{aligned}
I_{1+}^{2} & =\frac{a_{1-}}{a_{21} a_{2-}}+\frac{a_{+-}}{a_{2+} a_{2-}}-\frac{a_{1+}}{a_{21} a_{2+}}, \\
V_{1(+)}^{2} & =\frac{a_{1+}}{a_{21} a_{2+}}+\frac{a_{1-}}{a_{21} a_{2-}}-\frac{a_{+-}}{a_{2+} a_{2-}} .
\end{aligned}
$$

To study the anomalous dimension of the $(+)$ jet we should take all the gluon angles with respect to $\vec{n}_{+}$to be small. Then, $a_{1_{-}} \approx a_{2-} \approx a_{+-}(=2)$ and we come back to the planar gauge expressions. 


\section{Bibliography}

1. A.H. Mueller. Nucl. Phys., B213:85, 1983. Erratum quoted ibid., B241:141, 1984.

2. Yu.L. Dokshitzer and S.I. Troyan. Proceedings of the XIX Winter School of the LNPI, volume 1, page 144. Leningrad, 1984.

3. Yu.L. Dokshitzer, V.A. Khoze and S.I. Troyan. Coherence and physics of QCD jets. In A.H. Mueller, editor, Perturbative QCD, page 241. World Scientific, Singapore, 1989.

4. A. Bassetto, M. Ciafaloni and G. Marchesini. Phys. Rep., C100:201, 1983.

5. F. Fiorani, G. Marchesini and L. Reina. Nucl. Phys., B309:439, 1988.

6. G. Gustafson. Phys. Lett., 175B:453, 1986.

G. Gustafson and U. Pettersson. Nucl. Phys., B306:746, 1988. 


\section{Chapter 7}

\section{MLLA Hump-Backed Plateau}

\section{Contents}

7.1 Evolution Equation for Particle Spectra . . . . . . 222

7.2 Analytic Solution .............. 225

7.3 Developed Cascade and LPHD Hvpothesis . . . . 229

7.4 Mean Parton Multiplicitv . . . . . . . . 233

7.5 Shape of the Limiting Spectrum . . . . . . . 235

7.5.1 Gaussian Approximation . . . . . . . . . . . . . . 235

7.5.2 Mean Value of $\ln x \ldots \ldots$. . . . . . . . . 238

7.5.3 Width of the Hump . . . . . . . . . . . . . 239

7.5.4 Skewness and the Peak Position . . . . . . . . . . 241

7.6 Evaluation of the Limiting Spectrum . . . . . . . . 242

7.7 Preexponential Corrections to the Spectrum . . . 243 
7.8 Spectra in Jets with Restricted Opening Angles . 246

7.9 Phenomenology of Hump-Backed Plateau . . . 249

7.9 .1 Mean Particle Multiplicities . . . . . . . . . . . . 249

7.9 .2 Inclusive Particle Spectra . . . . . . . . . . . . 251

Bibliography . . . . . . . . . . . . . 257

Here we illustrate the Generating Functional technique with practically important and pedagogically instructive example, the inclusive energy spectrum of partons $B$ in jet $A$.

\subsection{Evolution Equation for Particle Spectra}

The MLLA Evolution Equation for particle spectra following directly from (6.18) reads:

$$
\frac{d}{d \ln \Theta} x \bar{D}_{A}^{B}(x, \ln E \Theta)=\sum_{C=q, \bar{q}, g} \int_{0}^{1} d z \frac{\alpha_{s}\left(k_{\perp}^{2}\right)}{2 \pi} \Phi_{A}^{C}(z)\left[\frac{x}{z} \bar{D}_{C}^{B}\left(\frac{x}{z}, \ln z E \Theta\right)\right],
$$

where $E, \Theta$ are energy and opening angle of a jet $A, \Phi_{A}^{C}$ stand for the regularized DGLAP kernels, $k_{\perp} \approx z(1-z) E \Theta \geq Q_{0}$. Introducing the notation

$$
\ell=\ln \frac{E}{k}=\ln \frac{1}{x}, \quad y=\ln \frac{k \Theta}{Q_{0}}, \quad Y=\ln \frac{E \Theta}{Q_{0}}=y+\ell,
$$


one arrives at a compact integro-differential equation

$$
\frac{d}{d Y} D_{\omega}(Y)=\int_{0}^{\infty} d \bar{\ell} e^{-\omega \bar{\ell}} \Phi(\bar{\ell}) \frac{\alpha_{s}(Y-\bar{\ell})}{2 \pi} D_{\omega}(Y-\bar{\ell})
$$

for the Mellin-transformed distributions

$$
D_{\omega}(Y)=\int_{0}^{1} \frac{d x}{x} x^{\omega}[x \bar{D}(x, Y)] .
$$

Equation (7.2) generalizes the LLA equation for moderate $x$ 's $(\omega \sim 1)$ over the region of parametrically small momenta $x \ll 1 \quad(\omega \sim 1 / \sqrt{Y} \ll 1)$.

Indeed, neglecting $\bar{\ell} \ll Y$ in the arguments of $\alpha_{s}$ and $D$ in (17.2) one would get the standard Evolution Equation

$$
\frac{d}{d Y} D_{\omega}(Y)=\frac{\alpha_{s}(Y)}{2 \pi} \widehat{\Phi}(\omega) D_{\omega}(Y) .
$$

As we have discussed in Chapter 团 diagonalization of the kernel matrix $\widehat{\Phi}$ results in the two "trajectories"

$$
\nu_{ \pm}(\omega)=\frac{1}{2}\left(\Phi_{G}^{G}+\Phi_{F}^{F} \pm \sqrt{\left(\Phi_{G}^{G}-\Phi_{F}^{F}\right)^{2}+8 n_{f} \Phi_{F}^{G} \Phi_{G}^{F}}\right)
$$

that determine the anomalous dimensions of two operators arising from mixing of $g$ and $q$ states:

$$
\gamma_{L L A}^{ \pm}\left(\omega, \alpha_{s}\right)=\frac{d}{d Y} \ln D^{ \pm}(\omega, Y)=\frac{\alpha_{s}(Y)}{2 \pi} \nu_{ \pm}(\omega) .
$$


At $x \ll 1$ the trajectory $\nu_{+}(\omega)$, singular at $\omega \equiv j-1=0$,

$$
\nu_{+}(\omega)=\frac{4 N_{c}}{\omega}-a+O(\omega), \quad a=\frac{11}{3} N_{c}+\frac{2 n_{f}}{3 N_{c}^{2}},
$$

gives the main contribution to $\bar{D}(x, Y)$.

The following chain of transformations makes it possible to express the nonlocal, in $Y$, MLLA equation (17.2) in terms of the known LLA trajectories with the differential operator $\widehat{\Omega}=\omega+d / d Y$ as an argument of $\Phi(j)$ :

$$
\frac{d}{d Y} D_{\omega}(Y)=\left(\int_{0}^{\infty} d \bar{\ell} \Phi(\bar{\ell}) \mathrm{e}^{-\bar{\ell}(\omega+d / d Y)}\right) \frac{\alpha_{s}(Y)}{2 \pi} D_{\omega}(Y)=\Phi(\widehat{\Omega}) \frac{\alpha_{s}(Y)}{2 \pi} D_{\omega}(Y)
$$

or after the diagonalization

$$
\frac{d}{d Y} D_{\omega}^{ \pm}(Y)=\nu_{ \pm}(\widehat{\Omega}) \frac{\alpha_{s}(Y)}{2 \pi} D_{\omega}^{ \pm}(Y)
$$

Using expansion (17.5) one obtains for the leading contribution

$$
\left(\omega+\frac{d}{d Y}\right) \frac{d}{d Y} D^{+}=4 N_{c} \frac{\alpha_{s}}{2 \pi} D^{+}-a\left(\omega+\frac{d}{d Y}\right) \frac{\alpha_{s}}{2 \pi} D^{+}
$$

Introducing anomalous dimension as follows

$$
D_{\omega}^{+}(Y)=D_{\omega}^{+}\left(Y_{0}\right) \cdot \exp \left\{\int_{Y_{0}}^{Y} d y \gamma_{\omega}\left(\alpha_{s}(y)\right)\right\}
$$

one finally arrives at the MLLA equation for $\gamma$ which clearly possesses the necessary RG property of locality:

$$
\left(\omega+\gamma_{\omega}\right) \gamma_{\omega}-\frac{4 N_{c} \alpha_{s}}{2 \pi}=-\beta\left(\alpha_{s}\right) \frac{d}{d \alpha_{s}} \gamma_{\omega}-a\left(\omega+\gamma_{\omega}\right) \frac{\alpha_{s}}{2 \pi}
$$


where $\beta\left(\alpha_{s}\right)=\frac{d}{d Y} \alpha_{s}(Y) \approx-b \alpha_{s}^{2} / 2 \pi, \quad b=\frac{11}{3} N_{c}-\frac{2}{3} n_{f}$.

The first term in the right hand side of (7.9) proportional to the $\beta$-function keeps trace of the running coupling effects while the second accounts for the "hard SL correction" to the DLA soft emission, see (7.5). Both prove to be $\sqrt{\alpha_{s}}$ corrections to the left hand side which is of the order $\alpha_{s}$. Within the DLA accuracy the known anomalous dimension comes immediately:

$$
\begin{aligned}
& \qquad \begin{array}{l}
\left(\omega+\gamma_{\omega}\right) \gamma_{\omega}-\gamma_{0}^{2}=0 \quad, \quad \gamma_{\omega}^{D L A}\left(\alpha_{s}\right)=\frac{1}{2}\left(-\omega+\sqrt{\omega^{2}+4 \gamma_{0}^{2}}\right) ; \\
\text { where } \quad \gamma_{0}^{2}=\gamma_{0}^{2}\left(\alpha_{s}\right) \equiv 4 N_{c} \frac{\alpha_{s}}{2 \pi} .
\end{array}
\end{aligned}
$$

This simple algebraic exercise replaces rather serious calculations one is forced to perform in the framework of conventional RG approach, summing up the series $\sum_{k=0}^{\infty} c_{k}\left(\alpha_{s} / \omega^{2}\right)^{k}$, which represented in fact expansion for the square root (7.10).

The Evolution Equation approach we are discussing proves to be even more efficient for the derivation of the MLLA result which follows from (7.9)

$$
\gamma=\gamma^{D L A}+\frac{\alpha_{s}}{2 \pi}\left[-\frac{a}{2}\left(1+\frac{\omega}{\sqrt{\omega^{2}+4 \gamma_{0}^{2}}}\right)+b \frac{\gamma_{0}^{2}}{\omega^{2}+4 \gamma_{0}^{2}}\right]+O\left(\alpha_{s}^{3 / 2}\right) .
$$

\subsection{Analytic Solution}

One can solve the differential equation (7.7) explicitly, reducing it to the confluent hypergeometric equation 1 for the product $\alpha_{s}(Y) D(Y)$. With account of initial conditions the solution reads:

$$
D^{+}(\omega, Y, \lambda)=\frac{\Gamma(A+1)}{\Gamma(B+2)} z_{1} z_{2}^{B}\left\{\Phi\left(-A+B+1, B+2,-z_{1}\right) \Psi\left(A, B+1, z_{2}\right)\right.
$$




$$
\left.+e^{z_{2}-z_{1}}(B+1) \Psi\left(A+1, B+2, z_{1}\right) \Phi\left(-A+B+1, B+1,-z_{2}\right)\right\} \cdot C_{i}^{f}
$$

where we have used the notation

$$
\begin{aligned}
& A=\frac{4 N_{c}}{b \omega}, \quad B=\frac{a}{b}, \quad \lambda=\ln \frac{Q_{0}}{\Lambda} \\
& z_{1}=\omega(Y+\lambda), \quad z_{2}=\omega \lambda .
\end{aligned}
$$

Indices $i$ and $f$ in (7.12) stand for the initial parton generating the jet $(i=q, g)$ and the final one spectrum which is studied $(f=q, g)$. In the leading approximation the coefficient functions $C_{i}^{g}$ are simply

$$
C_{g}^{g}=1, \quad C_{q}^{g}=\frac{C_{f}}{N_{c}}=\frac{4}{9} .
$$

Next-to-MLLA corrections to coefficient functions will be discussed below. Let us now consider (7.12) in more details. To reconstruct the $x$-distribution one has to perform the inverse Mellin transformation

$$
[x \bar{D}(x, Y)] \equiv \bar{D}(\ell, Y, \lambda)=\int_{\epsilon-i \infty}^{\epsilon+i \infty} \frac{d \omega}{2 \pi i} x^{-\omega} D^{+}(\omega, Y, \lambda)
$$

where the integral runs parallel to the imaginary axis to the right from all singularities of the integrand in the complex $\omega$-plane (if any). The confluent hypergeometric functions $\Phi(z)$ and $\Psi(z)$ have the following properties 1 .

- The differential equation for the confluent hypergeometric functions reads

$$
z \frac{d^{2} y}{d z^{2}}-(z-c) \frac{d y}{d z}-a y=0
$$


- The solution $y_{1}=\Phi$, regular in the complex $z$-plane, is given by power series

$$
\Phi(a, c ; z)=\sum_{n=0}^{\infty} \frac{(a)_{n}}{(c)_{n}} \frac{z^{n}}{n !}
$$

where $(x)_{n} \equiv x(x+1) \cdots(x+n-1)=\Gamma(x+n) / \Gamma(x)$.

- Its asymptotic behavior is

$$
\begin{aligned}
& \Phi(a, c ; z) \approx \frac{\Gamma(c)}{\Gamma(a)} e^{z} z^{a-c}, \quad \operatorname{Re} z \rightarrow+\infty \\
& \Phi(a, c ; z) \approx \frac{\Gamma(c)}{\Gamma(c-a)}(-z)^{-a}, \quad \operatorname{Re} z \rightarrow-\infty
\end{aligned}
$$

- The following Kummer relation holds for $\Phi(z)$ :

$$
\Phi(a, c ; z)=e^{z} \Phi(c-a, c ;-z) .
$$

- A second solution of (7.15)

$$
y_{2}=z^{1-c} \Phi(a-c+1,2-c ; z)
$$

can be combined with $y_{1}$ to get the solution regular at infinity:

$$
\begin{gathered}
\Psi(a, c ; z)=z^{1-c} \frac{\Gamma(c-1)}{\Gamma(a)} \Phi(a-c+1,2-c ; z)+\frac{\Gamma(1-c)}{\Gamma(a-c+1)} \Phi(a, c ; z) \\
\Psi(a, c ; z) \propto z^{-a} ; \quad|z| \rightarrow+\infty, \quad \arg (z)<\pi .
\end{gathered}
$$

- Being singular in the origin, $\Psi$ has a cut running along the real axis to the left from the branching point $z=0$. 
The expression (17.12) proves to be regular in the finite $\omega$-plant1 in spite of cuts in each of $\Psi\left(z_{1}\right)$ and $\Psi\left(z_{2}\right)$ terms. There are no poles due to the $\Gamma(A+1)=\Gamma\left(4 N_{c} / b \omega\right)$ factor despite the fact that poles might seem to occur at first glance. To see this one has to invoke (7.19) to get two equivalent expressions for $D^{+}$:

$$
\begin{aligned}
D^{+}(\omega, Y, \lambda) & =e^{-z_{1}}\left\{z_{1} \frac{A}{B(B+1)} \Phi\left(A+1, B+2 ; z_{1}\right) \Phi\left(A-B, 1-B ; z_{2}\right)\right. \\
& \left.+\left(\frac{z_{2}}{z_{1}}\right)^{B} \Phi\left(A-B,-B ; z_{1}\right) \Phi\left(A, B+1 ; z_{2}\right)\right\} \\
D^{+}(\omega, Y, \lambda) & =\frac{\Gamma(1-A)}{\Gamma(-B)}\left(-z_{1}\right)^{B}\left\{-\frac{1}{B} \Psi\left(-A,-B,-z_{1}\right) \Phi\left(A-B, 1-B, z_{2}\right)\right. \\
& \left.+e^{z_{2}-z_{1}} \Phi\left(A-B,-B, z_{1}\right) \Psi\left(1-A, 1-B,-z_{2}\right)\right\}
\end{aligned}
$$

As a consequence of analyticity of $D^{+}$in the $\omega$-plane which becomes explicit now with help of (7.21a), the result of $\omega$-integration would vanish when the integrand of (7.14)

$$
x^{-\omega} D^{+}=e^{\omega \ell} D^{+}
$$

decreases either at $\operatorname{Re} z \rightarrow+\infty$ or $\operatorname{Re} z \rightarrow-\infty$ where the $\omega$-contour can be moved. This happens indeed in two cases when

$\ell<0$, where for Re $\omega \rightarrow+\infty$ according to (7.17), (7.20) we have (pw denotes the power dependence on $\omega$ and the subscripts give references to the relevant representations for the integrand)

$$
e^{\omega \ell}\left\{\mathrm{pw}_{7.17 \mathrm{~b}} \cdot{ }^{\mathrm{pw}}[7.20]+e^{-\omega Y} \mathrm{pw}[7.20] \cdot{ }^{7.17 \mathrm{~b}}\right\}_{[7.12]} \sim e^{-\omega|\ell|} \rightarrow 0
$$

\footnotetext{
${ }^{1}$ This is a natural consequence of the analytical way, $\omega$ enters the differential equation (7.9) for the anomalous dimension
} 
$\ell>Y$, for $\operatorname{Re} \omega \rightarrow-\infty$

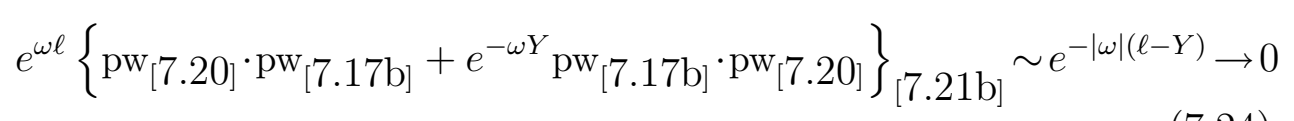

As one can easily see from (17.23), the second term in $D^{+}$in the form of (7.12) does not contribute to the answer in the whole physical region $0 \leq \ell \leq Y$, so we are left with

$$
\begin{gathered}
x \bar{D}_{i}^{f}(x, Y, \lambda)=\frac{4 N_{c}(Y+\lambda)}{b B(B+1)} \int_{\epsilon-i \infty}^{\epsilon+i \infty} \frac{d \omega}{2 \pi i} x^{-\omega} \Phi(-A+B+1, B+2,-\omega(Y+\lambda)) \cdot \mathcal{K}, \\
\text { where } \quad \mathcal{K} \equiv \mathcal{K}(\omega, \lambda)=\frac{\Gamma(A)}{\Gamma(B)}(\omega \lambda)^{B} \Psi(A, B+1, \omega \lambda) \cdot C_{i}^{f} .
\end{gathered}
$$

As we shall see below, the restored $x$-spectrum of partons exhibits the abovementioned "hump-backed" structure with a maximum at particle energies approaching asymptotically $\sqrt{E_{j e t}}$ :

$$
\ln \left(\frac{1}{x_{\max }}\right)=Y\left(\frac{1}{2}+a \sqrt{\frac{\alpha_{s}(Y)}{32 N_{c} \pi}}-a^{2} \frac{\alpha_{s}(Y)}{32 N_{c} \pi}+\ldots\right) .
$$

\subsection{Developed Cascade and LPHD Hypothesis}

Besides the jet energy $E$ and the QCD parameter $\Lambda$ the parton spectrum (7.25) contains one more dimensional quantity $Q_{0}$ that regularizes collinear 
divergences. This quantity represents the minimal value of the relative $k_{\perp}$ of decay products in jet evolution. $Q_{0}$ also bounds parton energies $E_{p}=x E \geq$ $k_{\perp} / \geq Q_{0}$, thus playing the role of an "effective mass" of a parton.

The choice of $Q_{0}$ value sets a formal boundary between two stages of jet evolution: the one of the parton branching processes, which is controlled by PT, and then the stage of non-PT transition into hadrons. In essence, "partons" and "hadrons" are complementary languages. So, if the theory of hadronization would exist, the result would be independent of the formal quantity $Q_{0}$ separating the two stages.

As a matter of fact, for large enough $Q_{0}$ the number of partons produced at recent energies is certainly small. So, one is forced to apply for some ad hoc hadronization model describing the multihadron production as the evolution "below $Q_{0}$ " of a partonic system with large invariant masses of all parton pairs. Unfortunately an experimental verification of such results look rather like a tuning of parameters of a phenomenological model than a test of QCD predictions.

At the same time, after an intense look at (7.25) an opportunity to make a model independent prediction may be found. The inclusive distribution of hadrons can be represented by a similar formula with the $\mathcal{K}$-factor replaced by the product

$$
\mathcal{K}^{h}(\omega)=\mathcal{K}(\omega, \lambda) \cdot C^{h}\left(\omega, \lambda ; M_{h}, J^{P C}\right),
$$

where $C^{h}(\omega)$ is a Mellin-transformed parton fragmentation function $C^{h}(z)$. In the kinematic region of relatively soft particles which we are interested in the essential values of $\omega$ under the integral in (7.25) are small: $\omega \ll 1$ (near the maximum - parametrically small: $\omega \sim \sqrt{\alpha_{s}(Y)}$ ). Therefore, to understand how hadronization affects the spectrum shape one needs to know the behavior of $\mathcal{K}^{h}$ at $\omega \rightarrow 0$.

(i) The singular behavior of the "matrix element" $\mathcal{K}^{h}$, say, $\mathcal{K}^{h}(\omega) \propto 1 / \omega+\ldots$ 
corresponds to the physical picture where each colored parton produces hadrons with a plateau-like energy distribution: $C^{h}(z) \propto 1 / z$. In such a case the dip at small $x$ which is characteristic for partonic spectra would never manifest itself in hadronic distributions.

(ii) The regular behavior $\mathcal{K} \rightarrow$ const corresponds to a local, in phase space, blanching and hadronization of partons which we have argued for in Chapter [1. In this case hadron and parton spectra prove to be similar.

It is the choice of the soft confinement scenario (ii) that forms the core idea of the Local Parton-Hadron Duality hypothesis 213 which stemmed from the preconfinement properties of QCD cascades 4 .

It is perhaps surprising to see the $x$-dependence of $x \bar{D}^{h}(x)$ being given completely by means of the PT evolution. Non-PT effects could smear parton distributions over a finite interval $\Delta \ln (1 / x) \sim 1$. This is however the higher order effect from the MLLA point of view. It is important to mention that one should not expect a one-to-one match between patrons and hadrons event by event, but only in their average behavior as well as in fluctuations around the average. Thus, the overall normalization factor remains the only arbitrary parameter. It may be fixed, e.g., by fitting the average multiplicity at some energy $E_{0}$. Whether or not present jet energies are sufficiently large for LPHD to be applied, is of course a question to experiment. So far, the experimental evidence suggests that LPHD works rather well.

The parton spectrum (7.25) has an interesting property supporting the LPHD hypothesis. Namely, at very high energies when the typical value of the product $\omega \lambda \sim \lambda / \sqrt{Y}$ becomes small, the shape of the spectrum becomes insensitive to the value of $Q_{0}$ :

$$
\mathcal{K}(\omega, \lambda) \approx \frac{2}{\Gamma(B)}\left(\frac{z_{0}}{2}\right)^{B} K_{B}\left(z_{0}\right)=\mathrm{const} \quad \text { at } \quad \omega \lambda \ll 1 ; \quad z_{0} \equiv \sqrt{\frac{16 N_{c} \lambda}{b}}
$$


with $K_{B}(z)$ the Mackdonald function (Modified Bessel function of the third kind 5 ). Therefore one can study the shape of energy spectra of well developed partonic cascades by use of a simplified version of the main formula (7.25) where one takes $\lambda=0$. In this limit any influence of the factor (7.27) disappears,

$$
\mathcal{K}(\omega, 0)=1,
$$

and, omitting the $C_{i}^{f}$ factor, we arrive at the expression which we shall refer to hereafter as the limiting spectrum $\bar{D}^{l i m}(\ell, Y) \equiv x \bar{D}^{l i m}(x, Y)$ :

$$
\begin{aligned}
\bar{D}^{l i m}(\ell, Y) & =\frac{4 N_{c} Y}{b B(B+1)} \int_{\epsilon-i \infty}^{\epsilon+i \infty} \frac{d \omega}{2 \pi i} x^{-\omega} \Phi(-A+B+1, B+2,-\omega Y) \\
& =\frac{4 N_{c} Y}{b B(B+1)} \int_{\epsilon-i \infty}^{\epsilon+i \infty} \frac{d \omega}{2 \pi i} x^{-\omega} e^{-\omega Y} \Phi(A+1, B+2, \omega Y) .
\end{aligned}
$$

These two representations are equivalent because of the Kummer relation (7.18). As we see, when the bremsstrahlung cascade is developed enough, the shape of resulting energy distribution of particles becomes insensitive to the processes occurring at the last steps of PT evolution (at $k_{\perp} \sim Q_{0}$ ). This observation may justify an attempt to provide the developed cascade not with an expensive increase of total energy $E$ but with decrease of $Q_{0}$, thus enhancing the responsibility of $\mathrm{PT} \mathrm{QCD}$ for jet evolution at recent energies 316 . In some sense the limiting choice $Q_{0} \approx \Lambda$ can be looked upon as a specific attempt to model confinement for production of light hadrons.

We are discussing here the simplest model-independent approach to make analytical predictions for hadron spectra. At the same time the LPHD concept can be quantitatively realized also in framework of various algorithmic 
models, see, e.g., Refs. [7 [10. Moreover, there exists a consistent field theoretic approach to the physics of color blanching that explicitly exhibits LPHD properties (the Gribov's confinement scenario [1]).

\subsection{Mean Parton Multiplicity}

Taking the $\omega=0$ moment in (17.25) one obtains an analytic expression for the parton multiplicity in terms of modified Bessel functions. The point $\omega=0$ is a somewhat peculiar one for the integrand of (7.25), where the argument $z$ of $\Phi(a, c ; z)$ vanishes while the first parameter $a$ according to (7.13) goes to infinity. To derive the true limit one has to recall the definition of the confluent hypergeometric function given by (7.16). Substituting values of parameters from (17.25) we get

$$
\Phi=1+\sum_{n=1}^{\infty} \frac{\left(4 N_{c} Y / b\right)^{n}}{(B+2)_{n} n !}\left(1-(B+1) \frac{\omega b}{4 N_{c}}\right) \cdots\left(1-(B+n) \frac{\omega b}{4 N_{c}}\right)
$$

Then at $\omega=0$ we arrive at

$$
\Phi=\sum_{n=0}^{\infty} \frac{\left(\frac{4 N_{c}}{b} Y\right)^{n}}{(B+2)_{n} n !}=\Gamma(B+2)\left(\frac{4 N_{c}}{b} Y\right)^{-(B+1) / 2} I_{B+1}\left(\sqrt{\frac{16 N_{c}}{b} Y}\right),
$$

where we have made use of the series expansion for the Modified Bessel function 5 :

$$
I_{\nu}(z)=\sum_{n=0}^{\infty} \frac{\left(\frac{z}{2}\right)^{2 n+\nu}}{\Gamma(\nu+n+1) n !} .
$$


Finally, the particle multiplicity corresponding to the limiting spectrum (7.28) reads

$$
\mathcal{N}=\Gamma(B)\left(\frac{z}{2}\right)^{-B+1} I_{B+1}(z)
$$

where

$$
z \equiv \sqrt{\frac{16 N_{c}}{b} Y}=2 Y \sqrt{4 N_{c} \frac{\alpha_{s}(Y)}{2 \pi}}=2 Y \cdot \gamma_{0}\left(\alpha_{s}\right) .
$$

Following the same lines the general formula for the parton multiplicity at fixed value of $\lambda$ can be derived:

$$
\mathcal{N}(Y, \lambda)=x_{1}\left(\frac{x_{2}}{x_{1}}\right)^{B}\left[I_{B+1}\left(x_{1}\right) K_{B}\left(x_{2}\right)+K_{B+1}\left(x_{1}\right) I_{B}\left(x_{2}\right)\right],
$$

with $x_{1}=\sqrt{\frac{16 N_{c}}{b}(Y+\lambda)}, \quad x_{2}=\sqrt{\frac{16 N_{c}}{b} \lambda}$. The first term in square brackets increases exponentially with $\sqrt{Y}$ while the second term decreases. Its role is to preserve the initial condition for the jet evolution, namely, $N(\lambda, \lambda)=1$. So, at $Y>\lambda$ it can in fact be neglected. At large values of $Y$ the limiting multiplicity has asymptotic behavior

$$
\mathcal{N} \propto Y^{-B / 2+1 / 4} \exp \sqrt{\frac{16 N_{c}}{b} Y}
$$

or, in terms of the running coupling,

$$
\ln \mathcal{N}=\sqrt{\frac{32 \pi N_{c}}{\alpha_{s}(Y)}} \frac{1}{b}+\left(\frac{B}{2}-\frac{1}{4}\right) \ln \alpha_{s}(Y)+O(1) .
$$

Let us remind the reader that because of the destructive soft gluon interference the first term in (7.35) is reduced by a factor $1 / \sqrt{2}$ relative to the case of incoherent QCD cascades. 


\subsection{Shape of the Limiting Spectrum}

Now we are going to study properties of the limiting spectrum (17.28). Since, as we have mentioned above, the developed PT cascade itself makes the shape of particle energy spectra infrared safe, it seems reasonable to start with comparing observed hadron spectra with the limiting parton distribution.

Equation (7.28) has been derived as asymptotically accurate. However, even for moderate energies it can be believed to give reasonable quantitative predictions because it represents the exact solution of the Evolution Equation (7.1) which accounts for the main physical ingredients of parton multiplication, namely, color coherence and energy balance in 2-particle QCD branching.

\subsubsection{Gaussian Approximation}

As we know from Chapter 5, at asymptotically high energies the inclusive distribution of partons is predicted to be Gaussian in $\ln x$, with the peak positioned at $\approx \frac{1}{2} \ln E$ and with the width proportional to $(\ln E)^{3 / 4}$. This hump-backed plateau is among the fundamental predictions of perturbative QCD. One may find the asymptotic shape of limiting distribution by the saddle-point evaluation of the spectrum moment representation treating $Y$ as a large parameter:

$$
\bar{D}(\ell, Y) \approx \mathcal{N}(Y)\left(\frac{36 N_{c}}{\pi^{2} b Y^{3}}\right)^{1 / 4} \exp \left[-\sqrt{\frac{36 N_{c}}{b}} \frac{\left(\ell-\ln 1 / x_{\max }\right)^{2}}{Y^{3 / 2}}\right] .
$$

This Gaussian distribution (7.36) can be written in a compact form

$$
\bar{D}(\ell, Y) \approx \frac{\mathcal{N}(Y)}{\sigma \sqrt{2 \pi}} \exp \left[-\frac{1}{2} \delta^{2}\right]
$$


in terms of the dispersion $\sigma$ and the mean value of $\ln (1 / x), \bar{\ell}$ (which coinsides with the peak position in this approximation)

$$
\begin{aligned}
\bar{\ell} & \equiv\langle\ell\rangle, \quad \delta \equiv(\ell-\bar{\ell}) / \sigma \\
\sigma^{2} & \equiv\left\langle(\ell-\bar{\ell})^{2}\right\rangle=\left\langle\ell^{2}\right\rangle-\bar{\ell}^{2} .
\end{aligned}
$$

As we know, the MLLA effects soften the spectrum by shifting the asymptotic DLA peak prediction $\bar{\ell}=\frac{1}{2} Y$ to larger values $\bar{\ell}=\left(\frac{1}{2}+c \sqrt{\alpha_{s}}+\cdots\right) Y$. The very shape of Gaussian is also affected by MLLA corrections. A transparent way to encode these effects not too far from the peak $(\delta \lesssim 1)$ has been proposed in Ref. 12 by means of a distorted Gaussian

$$
\bar{D}(\ell, Y) \approx \frac{\mathcal{N}(Y)}{\sigma \sqrt{2 \pi}} \exp \left[\frac{1}{8} k-\frac{1}{2} s \delta-\frac{1}{4}(2+k) \delta^{2}+\frac{1}{6} s \delta^{3}+\frac{1}{24} k \delta^{4}\right]
$$

with $s$ and $k$ the skewness and the kurtosis of the distribution 13 defined as:

$$
\begin{aligned}
s & \equiv \frac{\left\langle(\ell-\langle\ell\rangle)^{3}\right\rangle}{\sigma^{3}}=\kappa_{3}, \\
k & \equiv \frac{\left\langle(\ell-\langle\ell\rangle)^{4}\right\rangle}{\sigma^{4}}-3=\kappa_{4} .
\end{aligned}
$$

For the Gaussian distribution $s$ and $k$ vanish together with all other higher reduced cumulants $\kappa_{p}$ which are closely connected with structure of the Mellintransformed spectrum:

$$
\ln \bar{D}_{\omega}(Y)=\ln \mathcal{N}-\bar{\ell} \omega+\frac{1}{2} \sigma^{2} \omega^{2}+\sum_{p=3}^{\infty} \kappa_{p}(Y) \frac{(-\sigma \omega)^{p}}{p !} .
$$


The $\ell$-spectrum in terms of $\delta$ variable now takes the form

$$
\bar{D}(\ell, Y)=\frac{\mathcal{N}}{\sigma} \int \frac{d \nu}{2 \pi i} \exp \left\{\delta \cdot \nu+\frac{1}{2} \nu^{2}+\sum_{p=3}^{\infty} \kappa_{p}(Y) \frac{(-\nu)^{p}}{p !}\right\}
$$

The first two terms of the exponential generate (7.37), while an inclusion of $p=3,4$ contributions leads directly to the approximate expression (7.39). One can estimate $\kappa_{p}$ comparing expansion (7.41) with the general expression (7.8) for $\bar{D}_{\omega}$. The cumulants have the following connection to the anomalous dimension $\gamma_{\omega}$ :

$$
\begin{aligned}
\sigma^{2} & =\left.\int^{Y} d y\left(\frac{\partial}{\partial \omega}\right)^{2} \gamma_{\omega}\left(\alpha_{s}(y)\right)\right|_{\omega=0} \\
\kappa_{p} & =\left.\frac{1}{\sigma^{p}} \int^{Y} d y\left(-\frac{\partial}{\partial \omega}\right)^{p} \gamma_{\omega}\left(\alpha_{s}(y)\right)\right|_{\omega=0} .
\end{aligned}
$$

To estimate the MLLA $\gamma$ given by (7.11) we express it as

$$
\gamma_{\omega}\left(\alpha_{s}\right) \sim Y^{-1 / 2} \cdot\left[f_{1}\left(\omega^{2} Y\right)+\omega f_{2}\left(\omega^{2} Y\right)\right] .
$$

Taken together with an extra $Y$, coming from integration in (7.42), this leads to an estimate $(n \geq 1)$

$$
\begin{aligned}
\sigma^{2} & \sim Y \cdot Y^{-1 / 2} \cdot Y=Y^{3 / 2}, \\
\kappa_{2 n+2} & \sim\left(Y^{3 / 4}\right)^{-2 n-2} \cdot Y \cdot Y^{-1 / 2} \cdot Y^{n+1}=(\sqrt{Y})^{-n}, \\
\kappa_{2 n+1} & \sim\left(Y^{3 / 4}\right)^{-2 n-1} \cdot Y \cdot Y^{-1 / 2} \cdot Y^{n}=(\sqrt{Y})^{-n-1 / 2} .
\end{aligned}
$$

From (7.43) we conclude that the higher cumulants appear to be less significant for the shape of the spectrum in the hump region $\delta \lesssim 1$. It is straightforward to derive the moments of the limiting spectrum to show that, relative 
to the lowest order (DL) Gaussian spectrum, the peak in the $\ell$-distribution is shifted up (i.e. to the lower $x$ ), narrowed, skewed towards higher $x$ 's, and flattened, with tails that fall off more rapidly than a true Gaussian. The qualitative features of experimental data at present energies appear consistent with the expected properties.

\subsubsection{Mean Value of $\ln x$}

To evaluate the mean value of $\ln 1 / x$ one has to integrate by paths the expression

$$
\langle\ell\rangle \equiv\left\langle\ln \frac{1}{x}\right\rangle=\frac{1}{\Phi} \int_{0}^{\infty} d \ell \int_{\epsilon-i \infty}^{\epsilon+i \infty} \frac{d \omega}{2 \pi i}\left\{\frac{\partial}{\partial \omega} e^{\omega \ell}\right\} \Phi(-A+B+1, B+2,-\omega Y),
$$

where $\Phi$ stands for the multiplicity factor (17.30). Making use of (7.29) we get

$$
-\frac{\partial}{\partial \omega}\left[\left(1-(B+1) \frac{\omega b}{4 N_{c}}\right) \cdots\left(1-(B+n) \frac{\omega b}{4 N_{c}}\right)\right]_{\omega=0}=\frac{b}{4 N_{c}} \sum_{i=1}^{n}(B+i)
$$

as an extra factor in the series (7.30). Let us represent this sum in the convenient form

$$
\sum_{i=1}^{n}(B+i)=\frac{n(n+1)}{2}+n B=\frac{1}{2} n(n+B+1)+\frac{1}{2} B n .
$$

It is easy to see from (17.31) that introducing the factor $n(n+B+1)$ into (17.30) results in the same series for $I_{B+1}$ while the factor $n$ shifts the index 
of the Bessel function:

$$
\begin{gathered}
\sum_{n=1}^{\infty} \frac{(z / 2)^{2 n+\nu} n(n+\nu)}{\Gamma(\nu+n+1) n !}=\sum_{m=0}^{\infty} \frac{(z / 2)^{2 m+\nu+2}}{\Gamma(\nu+m+1) m !}=\left(\frac{z}{2}\right)^{2} I_{\nu}(z) \\
\sum_{n=1}^{\infty} \frac{(z / 2)^{2 n+\nu} n}{\Gamma(\nu+n+1) n !}=\sum_{m=0}^{\infty} \frac{(z / 2)^{2 m+(\nu+1)+1}}{\Gamma((\nu+1)+m+1) m !}=\frac{z}{2} I_{\nu+1}(z) .
\end{gathered}
$$

Finally for the mean $\ell$ we obtain the analytical expression

$$
\langle\ell\rangle=\frac{1}{I_{B+1}} \frac{b}{4 N_{c}}\left(\frac{z}{2}\right)^{2}\left[\frac{1}{2} I_{B+1}+\frac{2}{z} \frac{B}{2} I_{B+2}\right]=Y\left(\frac{1}{2}+\frac{B}{z} \frac{I_{B+2}}{I_{B+1}}\right) .
$$

In terms of the typical PT parameter $\gamma_{0}$ defined in (7.10b) (see also (7.33) $)$ our result reads

$$
\frac{\langle\ell\rangle}{Y}=\frac{1}{2}+\gamma_{0} \frac{a}{8 N_{c}} \cdot \frac{I_{B+2}(z)}{I_{B+1}(z)} .
$$

Neglecting $O\left(\gamma_{0}\right)$ terms in the large $Y$ limit one would get from (17.47) $\langle\ell\rangle=$ $\frac{1}{2} Y$ which is the known DLA result. The first $O\left(\gamma_{0} \sim \sqrt{\alpha_{s}}\right)$ correction one obtains approximating the ratio of Bessel functions as 1 at $z \propto \sqrt{Y} \gg 1$. This next-to-DLA Single Logarithmic correction softens the spectrum significantly. It takes into full account energy conservation, which affects the rate of particle multiplication overestimated by the DLA.

\subsubsection{Width of the Hump}

Following the same lines one can derive analytic expressions for arbitrary moments $\left\langle\ell^{m}\right\rangle$ determining the shape of the limiting spectrum. Let us illustrate the general technique with an example of $m=2$ which will tell us about 
the dispersion of the distribution (7.38b). Now we have to take the second derivative over $\omega$ near $\omega=0$ of the product (17.44) to get $\left\langle\ell^{2}\right\rangle$

$$
\left(\frac{\partial}{\partial \omega}\right)^{2} \prod_{k=1}^{n}\left(1-(B+k) \frac{\omega b}{4 N_{c}}\right)_{\omega=0}=\left(\frac{b}{4 N_{c}}\right)^{2} \cdot 2 \sum_{k=2}^{n} \sum_{i=1}^{k-1}(B+i)(B+k) .
$$

It is straightforward to calculate this double sum:

$$
2 \sum_{k=2}^{n} \sum_{i=1}^{k-1}(B+i)(B+k)=\frac{1}{4} \frac{n !}{(n-4) !}+\left(B+\frac{5}{3}\right) \frac{n !}{(n-3) !}+(B+1)(B+2) \frac{n !}{(n-2) !} .
$$

Analogously to the case of (17.45) it is convenient to rearrange this quartic polynomial in $n$ in the following form:

$$
\begin{gathered}
=\frac{1}{4}[n(n+B+1) \cdot(n-1)(n+B)]+\frac{B+\frac{1}{3}}{2}[n(n+B+1) \cdot(n-1)] \\
+\frac{B\left(B+\frac{1}{3}\right)}{4}[n(n+B+1)]-\frac{B\left(B+\frac{1}{3}\right)(B+2)}{4}[n] .
\end{gathered}
$$

According to the "shifting rules" (17.46) we then arrive at

$$
\left\langle\ell^{2}\right\rangle=Y^{2}\left[\frac{1}{4}+\frac{B\left(B+\frac{1}{3}\right)}{z^{2}}+\frac{\left(B+\frac{1}{3}\right)}{z}\left(1-\frac{2 B(B+2)}{z^{2}}\right) \frac{I_{B+2}(z)}{I_{B+1}(z)}\right] .
$$

Now we can calculate the dispersion subtracting from (17.49) the square of $\langle\ell\rangle$ given by (7.47). The leading contribution cancels and one is left with

$$
\frac{\sigma^{2}}{Y^{2}}=\frac{1}{3 z} \frac{I_{B+2}(z)}{I_{B+1}(z)}+\frac{B}{3 z^{2}}+O\left(\frac{1}{z^{3}}\right)=\frac{1}{3 z}-\frac{1}{2 z^{2}}+O\left(\frac{1}{z^{3}}\right) .
$$


Here, to obtain the $1 / z^{2}$ correction, we made use of the asymptotic expansion

$$
I_{\nu}(z)=\frac{e^{z}}{\sqrt{2 \pi z}} \sum_{m=0}^{\infty}\left(\frac{1}{2 z}\right)^{m} \frac{\Gamma\left(\frac{1}{2}+m+\nu\right)}{m ! \Gamma\left(\frac{1}{2}-m+\nu\right)} \approx \frac{e^{z}}{\sqrt{2 \pi z}}\left[1-\frac{\nu^{2}-\frac{1}{4}}{2 z}\right],
$$

which gives the difference from unity of the ratio of Bessel functions:

$$
\frac{I_{B+2}(z)}{I_{B+1}(z)}=1-\frac{(2 B+3)}{2 z}+\ldots
$$

\subsubsection{Skewness and the Peak Position}

From the expression (7.39) for distorted Gaussian one can see that the nonzero value of skewness leads to a noticeable and calculable splitting between $\ell_{\max }$ and $\bar{\ell}$ :

$$
\delta_{\max } \approx-\frac{s}{2} ; \quad \ln \left(\frac{1}{x_{\max }}\right)=\bar{\ell}-\frac{1}{2} s \cdot \sigma=\bar{\ell}+O(1) .
$$

Skewness vanishes in DLA, as all higher odd cumulants do, so that only one SL correction term from the MLLA expansion (17.11) for the anomalous dimension contributes to (7.42b):

$$
\frac{d}{d Y}\left(\sigma^{3} s\right)=-\left.\frac{a}{2}\left(-\frac{\partial}{\partial \omega}\right)^{3} \frac{\omega}{\sqrt{\omega^{2}+4 \gamma_{0}^{2}}}\right|_{\omega=0}=-\frac{3 a}{16 N_{c}} \cdot \frac{1}{4 \gamma_{0}} .
$$

Integrating over $y$ and making use of (17.50) we arrive at

$$
\begin{aligned}
\frac{1}{4} \int_{0}^{Y} d y \frac{1}{\gamma_{0}} & =\frac{1}{4} \sqrt{\frac{b}{4 N_{c}}} \int_{0}^{Y} d y \sqrt{y}=Y^{2} \frac{1}{3 z} \approx \sigma^{2} \\
s & =\frac{1}{\sigma^{3}}\left(-\frac{3 a}{16 N_{c}} \cdot \sigma^{2}\right)=-\frac{3 a}{16 N_{c}} \frac{1}{\sigma}
\end{aligned}
$$


which results in an interesting simply testable QCD prediction revealing the net subleading MLLA effects in parton cascades:

$$
\ell_{\max }-\bar{\ell} \approx \frac{1}{2} \frac{3 a}{16 N_{c}} \equiv \frac{11}{32}+\frac{n_{f}}{16 N_{c}^{3}}=0.351(0.355) \text { for } n_{f}=3(5) .
$$

To find the absolute value of the peak position we first invoke expression (17.47) for the mean $\ell$

$$
\bar{\ell}=Y\left\{\frac{1}{2}+\frac{B}{z} \cdot \frac{I_{B+2}(z)}{I_{B+1}(z)}\right\} \approx Y\left\{\frac{1}{2}+\frac{B}{z}-\frac{B(2 B+3)}{2 z^{2}}+\cdots\right\},
$$

which, taken together with skewness-splitting (7.52), gives the asymptotic expansion (7.26) for the MLLA-improved $\ln \left(1 / x_{\max }\right)$ :

$$
\ln \frac{1}{x_{\max }}=Y\left[\frac{1}{2}+\sqrt{\frac{c}{Y}}-\frac{c}{Y}+O\left(Y^{-3 / 2}\right)\right]
$$

with $\quad c=\frac{a^{2}}{16 N_{c} b}=\frac{11}{48} \frac{\left(1+2 n_{f} / 11 N_{c}^{3}\right)^{2}}{1-2 n_{f} / 11 N_{c}}=0.29(0.35) \quad$ for $n_{f}=3(5)$.

\subsection{Evaluation of the Limiting Spectrum}

Equation (17.25) is badly suited for numerical evaluation. To get practically useful formulae we exploit the following integral representation for the confluent hypergeometric function $\Phi$ :

$$
\frac{1}{\Gamma(c)} \Phi(a, c, x)=x^{1-c} \int_{\gamma-i \infty}^{\gamma+i \infty} \frac{d t}{2 \pi i} e^{x t} t^{-c}\left(\frac{t}{t-1}\right)^{a}, \quad \gamma_{0}>1
$$

The path parallel to the imaginary axis can be replaced by an integration around the cut $0 \leq t \leq 1$. Substituting (7.54) for the integrand in the second 
form of the representation (7.28) for the limiting spectrum, we change the order of $\omega$ and $t$ integrations. Integral over $\omega$ results then in the Modified Bessel function $I_{B}$ for $t>\zeta \equiv 1-\ell / Y$. For smaller values of $t$, as can be easily seen, the $\omega$-integral vanishes. Finally, introducing the complex variable $\alpha$ according to

$$
\alpha=\frac{1}{2} \ln \frac{t}{t-1} ; \quad t=\frac{e^{\alpha}}{2 \sinh \alpha}, \quad d t=\frac{d \alpha}{2 \sinh ^{2} \alpha},
$$

we arrive at the following convenient expression 2

$$
\begin{aligned}
\bar{D}^{l i m}= & \frac{4 N_{c}}{b} \Gamma(B) \int_{-\frac{\pi}{2}}^{\frac{\pi}{2}} \frac{d \tau}{\pi} e^{-B \alpha}\left[\frac{\cosh \alpha+(1-2 \zeta) \sinh \alpha}{\frac{4 N_{c}}{b} Y \frac{\alpha}{\sinh \alpha}}\right]^{B / 2} \\
& \cdot I_{B}\left(\sqrt{\frac{16 N_{c}}{b} Y \frac{\alpha}{\sinh \alpha}[\cosh \alpha+(1-2 \zeta) \sinh \alpha]}\right)
\end{aligned}
$$

where $\alpha=\alpha_{0}+i \tau$ and $\alpha_{0} \quad$ is determined by

$$
\tanh \alpha_{0}=2 \zeta-1 \text {. }
$$

\subsection{Preexponential MLLA Corrections to the Limiting Spectrum}

Account of the subleading $\mathcal{O}\left(\sqrt{\alpha_{s}}\right)$ MLLA terms in the "preexponential" coefficient function factors $C_{i}^{f}$ in (7.25), provides a numerically small correction to the multiplicity ratio and leads also to some controllable change in the 
shape of particle spectra in $q$ and $g$ jets. Indeed, the residues of the leading "+" trajectory determining the yield of gluons-partons in the quark and gluon jets read

$$
\begin{aligned}
& C_{q}^{g}=\frac{\Phi_{F}^{G}}{\nu_{+}-\nu_{-}} \approx \frac{\frac{4 C_{F}}{j}-3 C_{F}}{\left(\frac{4 N_{c}}{j}-a\right)-\left(-\frac{2}{3} n_{f} \frac{2 C_{F}}{N_{c}}\right)} \approx\left[1+\Delta_{q}^{g} j\right] \frac{C_{F}}{N_{c}}, \\
& C_{g}^{g}=\frac{\nu_{+}-\Phi_{F}^{F}}{\nu_{+}-\nu_{-}} \approx \frac{\frac{4 N_{c}}{j}-a}{\left(\frac{4 N_{c}}{j}-a\right)-\left(-\frac{2}{3} n_{f} \frac{2 C_{F}}{N_{c}}\right)} \approx\left[1+\Delta_{g}^{g} j\right],
\end{aligned}
$$

where $\left(n_{f}=3\right)$

$$
\begin{array}{ll}
\Delta_{g}^{g}=-\frac{1}{3} n_{f} \frac{C_{F}}{N_{c}^{2}} & =-\frac{4}{27}, \\
\Delta_{q}^{g}=\Delta_{0}+\Delta_{g}^{g} & =+\frac{1}{27} ; \\
\Delta_{0} \equiv \frac{a-3 N_{c}}{4 N_{c}} & =+\frac{5}{27} .
\end{array}
$$

Additional factor $\Delta \cdot j$ in the integrand of (77.25) generates the $\sqrt{\alpha_{s}}$ correction which can be taken into account by the following trick using $\widehat{\Omega}=\omega+\frac{d}{d Y}$ (cf. (7.6)):

$$
\begin{aligned}
& \bar{D}_{i}^{g}(\ell, Y)=\int_{\epsilon-i \infty}^{\epsilon+i \infty} \frac{d \omega}{2 \pi i} e^{\ell \omega}\left[1+\Delta_{i}^{g} \cdot \widehat{\Omega}\right] \bar{D}^{l i m}(\omega, Y)= \\
& {\left[1+\Delta_{i}^{g}\left(\frac{\partial}{\partial \ell}+\frac{\partial}{\partial Y}\right)\right] \bar{D}^{l i m}(\ell, Y) \approx \bar{D}^{l i m}\left(\ell+\Delta_{i}^{g}, Y+\Delta_{i}^{g}\right) .}
\end{aligned}
$$


When calculating the gluon multiplicity, the $\frac{\partial}{\partial \ell}$ term in (7.58) vanishes and one is left with

$$
\begin{aligned}
& N_{g}=\left[1+\Delta_{g}^{g} \frac{\partial}{\partial Y}\right] \mathcal{N}(Y), \\
& N_{q}=\left[1+\Delta_{q}^{g} \frac{\partial}{\partial Y}\right] \mathcal{N}(Y) \cdot \frac{C_{F}}{N_{c}} .
\end{aligned}
$$

The derivative of $\ln \mathcal{N}(Y)$ is nothing but the value of the anomalous dimension $\gamma_{\omega}\left(\alpha_{s}\right)$ at $\omega=0$. Since we are considering now the correction term, $\gamma_{0}$ should be taken in the DLA according to (7.10). Thus, the ratio of the parton multiplicities in $q$ and $g$ jets takes the form

$$
\frac{N_{c}}{C_{F}} \cdot \frac{N_{q}}{N_{g}}=\frac{\left[1+\Delta_{q}^{g} \gamma_{0}\right]}{\left[1+\Delta_{g}^{g} \gamma_{0}\right]} \approx 1+\Delta_{0} \gamma_{0}=1+\frac{a-3 N_{c}}{4 N_{c}} \sqrt{4 N_{c} \frac{\alpha_{s}(Y)}{2 \pi}} .
$$

This next-to-leading correction had been obtained first in Refs. 1415. Notice, that because of factorization of the LLA residues the ratio (7.59) remains unchanged when one takes quarks as final partons instead of gluons.

Corrections to the parton spectra can be described according to (77.58) as a shift of the limiting distribution to a slightly different jet energy 12 . Namely, one gets the spectrum of gluons with fixed energy $E_{h}$ for a given jet energy $E$ with account of $\sqrt{\alpha_{s}}$ corrections just by taking the distribution $\bar{D}^{l i m}\left(\ln \left(E_{\text {lim }} / E_{h}\right), \ln \left(E_{\text {lim }} / \Lambda\right)\right)$ given by (77.28), (77.55) at

$$
\begin{aligned}
E_{\text {lim }} & =E \exp \left(\Delta_{q}\right) \approx 1.04 E & & \text { for } q \text {-jet } \\
\text { and } & E_{\text {lim }}=E \exp \left(\Delta_{g}\right) \approx 0.86 E & & \text { for } g \text {-jet . }
\end{aligned}
$$

The relative "shift" of $q$ and $g$ jets is determined by the same value $\Delta_{0}=5 / 27$ as the multiplicity ratio. Strictly speaking, only such relative features of $q$ and 
$g$ jets are under the quantitative perturbative control at present. To derive absolute predictions with the accuracy including $\sqrt{\alpha_{s}}$ effects, one needs to go beyond the MLLA scope.

The same remark holds true for the next-to-next-to-leading correction to the hump position. Scaling the value of $\Lambda$ by a factor $C$ would shift the constant term in (7.26), (7.53) by $\ln C$. Therefore, to guarantee the next-tonext-to-leading terms one has to define precisely the energy scale by fixing the normalization scheme.

\subsection{Spectra of Particles within Restricted Jet Opening Angles}

It is noteworthy that according to (7.26) the energy $E_{\max }$ at the peak grows rather slowly with the jet energy $E$; even at $E \approx 1.5 \mathrm{TeV}$, its value reaches only $E_{\max } \approx 3 \mathrm{GeV}$. To explore the coherent origin of the hump-backed particle spectrum and in an attempt to study the depletion in its soft part for jets produced in hadronic collisions, it proves to be important to look at particles restricted to lie within a particular opening angle with respect to the jet 16. For example, one might consider the energy distribution of particles accompanying the production of an energetic particle and lying within a cone of half-angle $\Theta$ about the direction of the trigger particle momentum.

Parton cascades in these situations will populate mainly the region

$$
\frac{m_{h}}{\sin \Theta}<E_{h}<E
$$

with $E_{h}$ and $m_{h}$ being the energy and the mass of the observed particle $\left(m_{h} \gtrsim \Lambda\right)$. The maximum of the distribution, in $E_{h}$, is now forced to larger 
energies

$$
\ln \frac{E_{\max }}{m_{h}} \approx \frac{1}{2} \ln \frac{E}{m_{h} \sin \Theta}-B\left(\sqrt{\frac{b}{16 N_{c}} Y_{\Theta}}-\sqrt{\frac{b}{16 N_{c}} \ln \frac{m_{h}}{\Lambda}}\right) .
$$

If one chooses $\Theta$ moderately small and varies $E$, coherence will give a moving peak in accordance with the relation

$$
\frac{E}{E_{\max }} \frac{d E_{\max }}{d E}=\frac{1}{2}-B \sqrt{\frac{b}{64 N_{c} Y_{\Theta}}} ; \quad Y_{\Theta}=\ln \frac{E \sin \Theta}{\Lambda} .
$$

The angular cut $\Theta$ is especially useful for jets produced in hadronic collisions, since one is able to eliminate much of the soft background.

Fig. [7.1 illustrates the energy distribution of charged hadrons in different cones $\Theta$ around the jet axis at $E / \Lambda=10^{4}$. The "restricted cone" inclusive particle distribution $D^{\Theta}(x, E)$ could be also defined in terms of the energymultiplicity correlation to get rid of the bias effect caused by determination of the jet axis, see (9.3) below. 


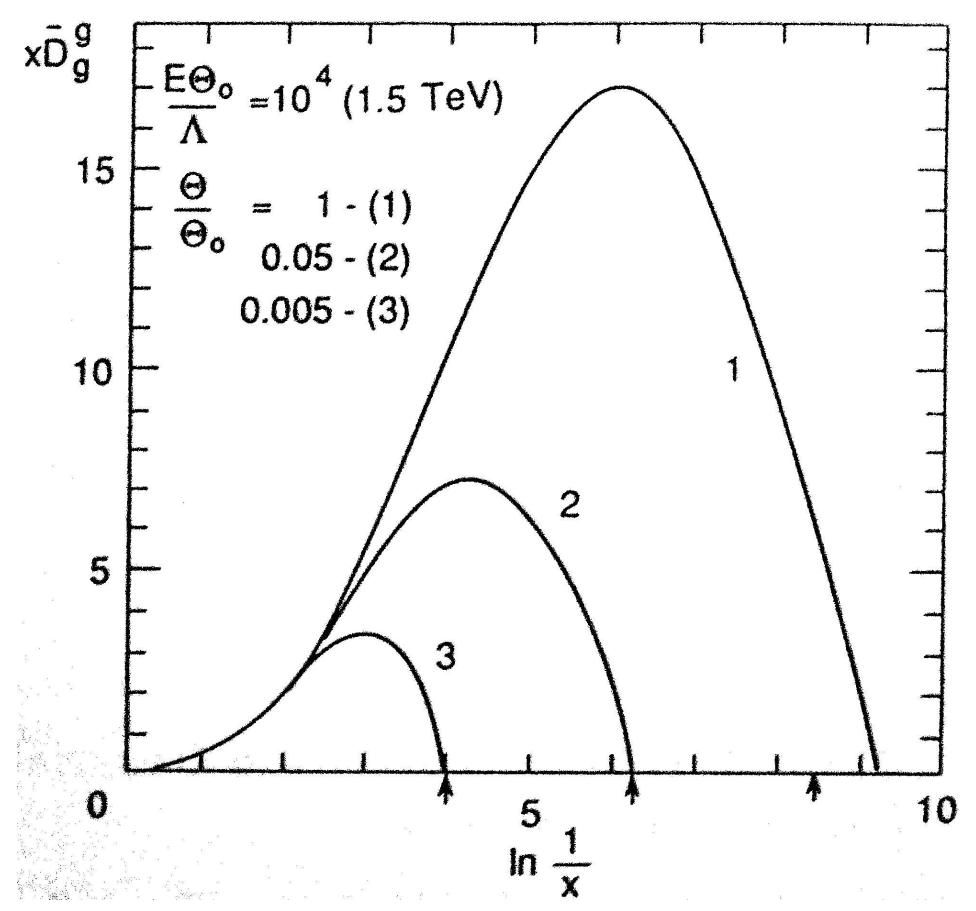

Figure 7.1: Dependence of the energy spectrum of partons in a gluon jet with $E / \Lambda=10^{4}$ on the aperture of the registered particle flow 17 . The narrower is the registration cone, the harder are the particles within this cone. The value of energy, given in brackets, corresponds to $\Lambda=155 \mathrm{MeV}$. 


\subsection{Phenomenology of Hump-Backed Plateau}

In this Section we discuss average multiplicities and spectra of hadrons as they are seen in present day experiments.

\subsubsection{Mean Particle Multiplicities}

The average multiplicity and multiplicity fluctuations (described by multiplicity moments) are among the most characteristic QCD predictions that depend on the main features of partonic cascades. The average multiplicity of gluons in a gluon jet is given by the MLLA limiting formulae (7.32) with asymptotic behavior of (7.35).

In the framework of the LPHD concept the MLLA analytical expressions can be directly applied for a description of the hadron inclusive distributions with the values of effective parameter $\Lambda$ and the normalization factors $K^{h}$ determined from comparison with the data at present energies. A rather reasonable phenomenology can be done for hadroproduction in QCD jets taking

$$
N_{g}^{h}=\mathcal{K}^{h} \cdot \mathcal{N}_{g}^{g}
$$

with $\mathcal{N}_{g}^{g}$ given by the limiting expression (17.32). The average multiplicity of charged particles in $e^{+} e^{-}$annihilation is asymptotically given by

$$
N_{e^{+} e^{-}}^{c h}(W)=2 \cdot \frac{4}{9} \cdot \mathcal{K}^{c h} \mathcal{N}_{g}^{g}(Y)
$$

where $Y=\ln W / 2 \Lambda$. The first comparison 36 of the $\pi^{ \pm}$data at PETRA/PEP energies with the limiting MLLA formulae has demonstrated a good agreement with $\Lambda$ numerically close to $m_{\pi}$. It has also been noticed 
that the relative yields of hadrons of various species $(p, K, \ldots)$ can be reasonably simulated by cutting the development of partonic cascade, leading to the production of a hadron $h$, by the value $Q_{0}$ increasing with $M_{h}(\lambda>0)$ at the same value of $\Lambda$.

Let us emphasize that the distributions of different hadrons should become similar to one another and to the theoretical "limiting" patron spectrum asymptotically at $Y \rightarrow \infty$. At present energies a number of asymptotically vanishing effects might influence hadronic spectra, the effect of final hadron masses among them.

The experimental results on charged particle multiplicity for $e^{+} e^{-}$collisions (see Refs. [18, 21] and references therein) are well described by the limiting formulae (7.32), (7.35). Taking this agreement as a testing ground, one can predict the dynamics of the multiplicity growth.

Contrary to the case of light particles, heavy particle content of QCD jets can be calculated by means of PT QCD directly without invoking any (though plausible) conjectures. The heavy quark multiplicity in gluon jet is given by the following formulae accounting for both first order result relevant at moderate energies $\left(W^{2} \gtrsim m_{Q}^{2}\right)$ and high energy cascading factor (see Ref. [22]):

$$
N_{g}^{(Q, \bar{Q})}=\frac{1}{3 \pi} \int_{4 m_{Q}^{2}}^{W^{2}} \frac{d k^{2}}{k^{2}} \alpha_{s}\left(k^{2}\right)\left[1+\frac{2 m_{Q}^{2}}{k^{2}}\right] \sqrt{\frac{1}{4}-\frac{m_{Q}^{2}}{k^{2}}} \mathcal{N}_{g}^{g}\left(W^{2}, k^{2}\right),
$$

where $\mathcal{N}_{g}^{g}\left(W^{2}, k^{2}\right)$ is the multiplicity of virtual gluons as given by (77.34) with $Y=\ln W / \Lambda, \lambda=\ln \sqrt{k^{2}} / \Lambda$. 


\subsubsection{Inclusive Particle Spectra}

Characteristics of an individual jet are best measured in the process $e^{+} e^{-} \rightarrow$ hadrons. Thus, the inclusive particle spectrum for $e^{+} e^{-}$annihilation is the sum of two $q$-jet distributions

$$
\frac{1}{\sigma} \frac{d \sigma}{d\left(\ln \frac{1}{x}\right)}=2 \bar{D}_{q}^{h}(\ell, Y) \approx \frac{8}{9} \bar{D}_{g}^{h}(\ell, Y) .
$$

A strong support for the PT scenario of multihadron production in jets came recently from the LEP data 2123 on inclusive momentum distribution of charged particles at the $Z^{0}$. To observe the predicted energy evolution these data were combined with the results at lower energies. Fig. 7.2 shows the OPAL and TASSO 24 data on the $\ln 1 / x_{p}$ distribution $\left(x_{p}=2 p_{h} / W\right)$ together with the prediction of the formulae (7.55). Motivating by LPHD, the limiting gluon spectrum was multiplied by an overall normalization factor $\mathcal{K}^{c h}$. An agreement between the data and the MLLA prediction can be easily improved 25 by taking into account finite hadron mass effects at $p_{h} \sim m_{h}$.

Fit to the OPAL data using (17.55) gave the following values of the only free parameters

$$
\Lambda=0.253 \pm 0.030 \mathrm{GeV}, \quad \mathcal{K}^{c h}=1.28 \pm 0.01 .
$$

These results confirm the QCD cascading picture of the multiple hadroproduction.

Especially spectacular is the energy evolution of the peak position given by (7.26) and shown in Fig. 17.3, which depends on the value of $\Lambda$ only, being independent of the normalization factor. It is interesting to mention that when fitting the energy evolution of the position of the maximum to (17.26) even the magnitude of the third constant term $-Y a^{2} \frac{\alpha_{s}(Y)}{32 N_{c} \pi} \approx-0.3$, is rather well reproduced by the data. 


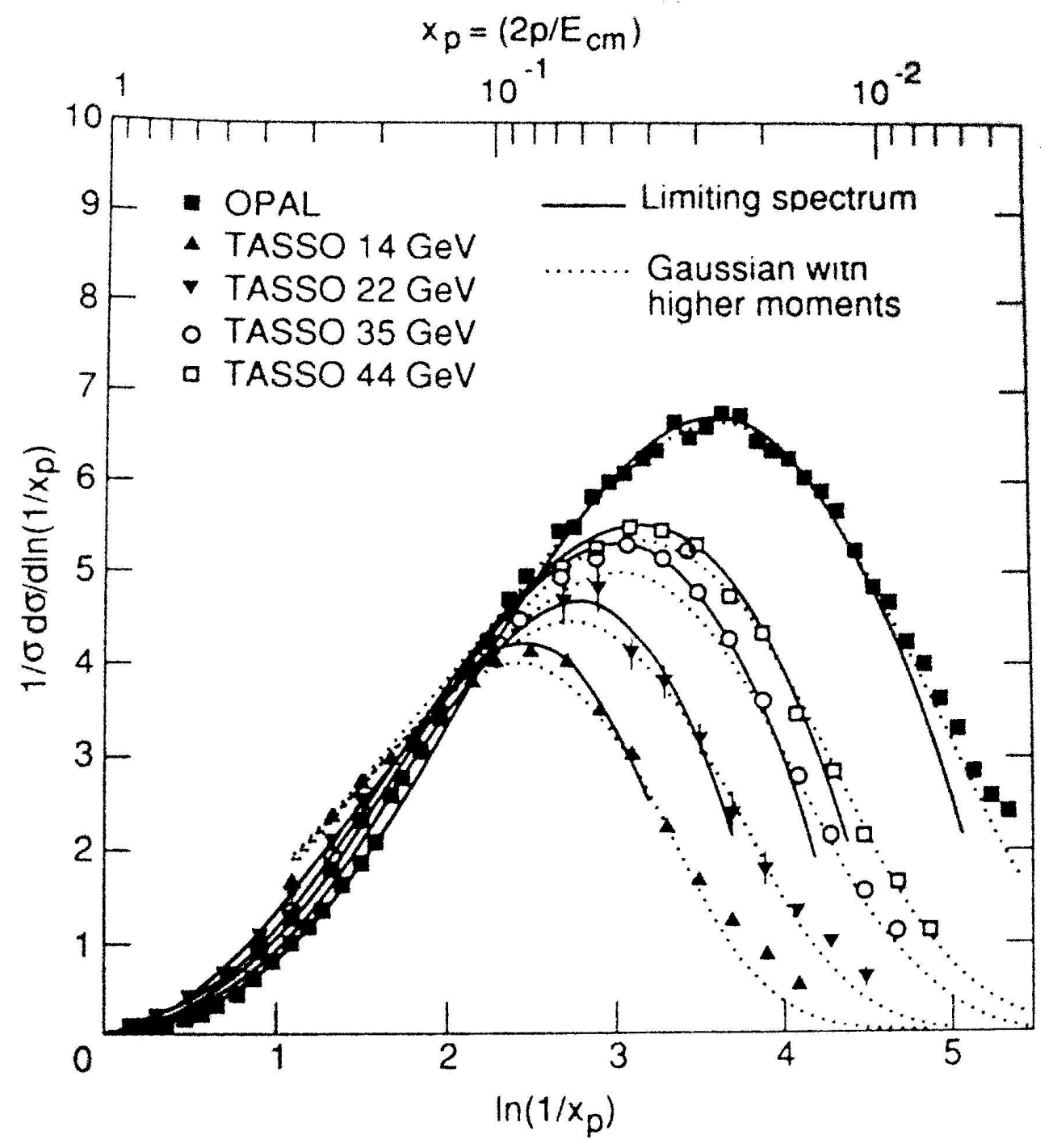

Figure 7.2: $\ln 1 / x_{p}$ distributions of charged hadrons at $W=14,22,35,44$ and $91 \mathrm{GeV}$ compared with analytical MLLA formula (7.55) and the distorted Gaussian (7.39). 


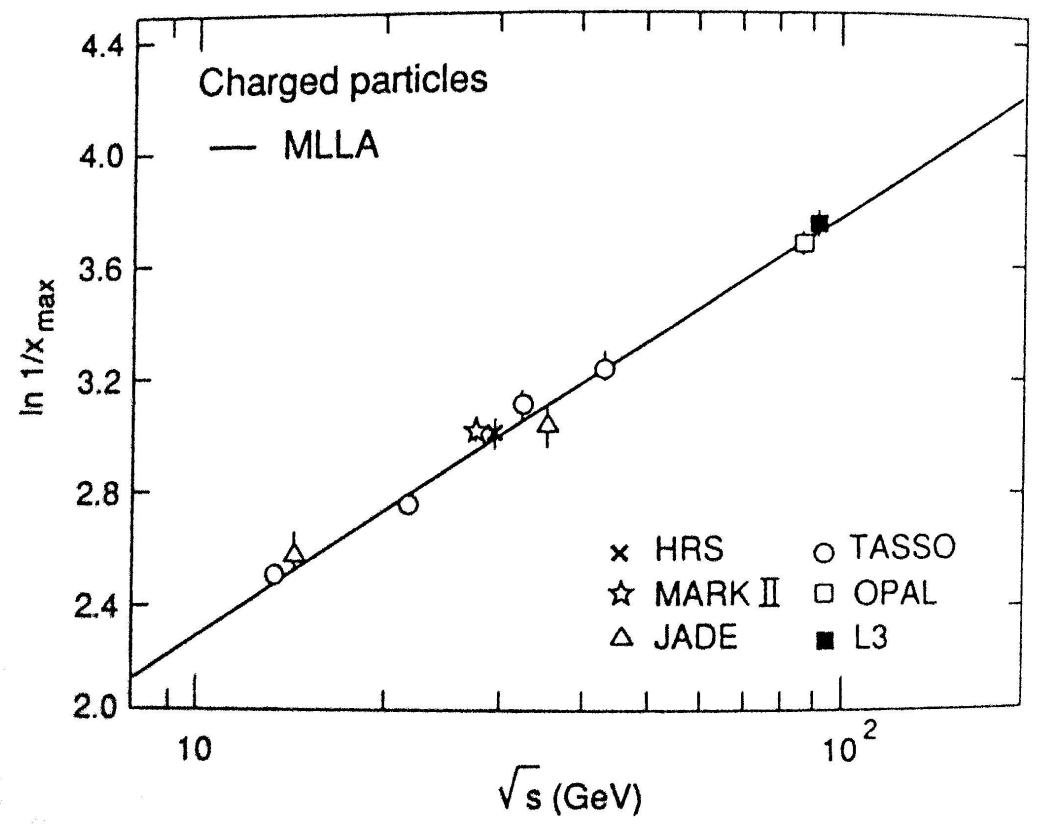

Figure 7.3: Energy evolution of the peak position compared to the formula (7.26).

Let us make a few comment concerning particle spectra. It looks rather challenging that even in the finite- $x_{p}$ region $\left(x_{p} \sim 1\right)$ the data agree with formula (17.28) derived as a true solution of the MLLA Evolution Equations for small particle momenta fractions $x_{p} \ll 1$. Such an agreement proves to be natural, though accidental. This coincidence stems from the fact that in terms of the Mellin-transformed distribution (7.3) the main contribution to $\bar{D}(\ell, Y)$ at $\ell \gg 1$ comes from parametrically small values of $\omega$ where the 
approximation (7.5) for the leading "trajectory", namely,

$$
\nu_{+}(\omega)=\frac{4 N_{c}}{\omega}-a+O(\omega) \quad\left(\approx \frac{12}{\omega}-\frac{101}{9} \quad \text { for } n_{f}=3\right)
$$

was made to derive the limiting spectrum (77.28). This approximation, however, proves to mimic reasonably well the behavior of $\gamma_{\omega}$ at $\omega \sim 1$ also, which region is responsible for $x \sim 1(\ell \sim 1)$. Indeed, at $\omega=1$ (77.66) gives $\nu=12-101 / 9=7 / 9$ which is not far from the exact value $\nu=0$ (following from the momentum conservation). At larger $\omega(7.66)$ becomes negative (as the true $\gamma$ does), thus imitating the scaling violation at $x \rightarrow 1$.

An even more intriguing problem comes from the observed 21 decrease by $14 \%(8 \%)$ of $\mathcal{K}(Y)$ as the energy is increasing from $14(22)$ to $91 \mathrm{GeV}$. Noticing that the systematic error to the absolute normalization for the OPAL (TASSO) data was $\pm 5 \%( \pm 7 \%)$, one could nevertheless try to take this observation seriously and discuss its consequences. In the LPHD framework parton $\rightarrow$ hadron conversion coefficients have to be $Y$ - and $x$-independent if hadronization occurs locally in the phase-space. However, the observed distribution of charged particles is in fact a mixture of different particles

$$
\bar{D}^{c h}=\bar{D}^{\pi^{ \pm}}+\bar{D}^{K^{ \pm}}+\bar{D}^{p \bar{p}}+\ldots,
$$

spectra of which prove to be not exactly similar: the position of the hump in $\bar{D}^{h}$ stiffens with $m_{h}$ increasing. This can cause in principal some $\mathcal{K}^{c h}(Y)$ dependence at fixed values of $\mathcal{K}^{ \pm}, \mathcal{K}^{K^{ \pm}}, \mathcal{K}^{p}$.

Stiffening of massive hadron spectra can be simulated by truncating parton cascades at the value $Q_{0}^{e f f}$ increasing with $m_{h}$. Corresponding procedure together with analytical results for the shape parameters determining the distorted Gaussian for truncated parton cascades $(\lambda>0)$ can be found in Ref. [26]. 
Secondly, a theoretical explanation of some preasymptotic $\mathcal{K}(Y)$ decrease could be connected with the necessity to supply (7.28) with an extra factor 25

$$
\left(1+\frac{2 n_{f}}{3 \sqrt{N_{c}}} \sqrt{\frac{\alpha_{s}(Y)}{2 \pi}}+O\left(\alpha_{s}(Y)\right)\right)
$$

accounting for the yield of the soft sea $q \bar{q}$ pairs which was neglected in the MLLA formula (7.28) written for the spectrum of gluons-partons exclusively. To clear up this situation as well as for a more profound study of LPHD picture conjectures, the measurements of identified hadron spectra are of substantial importance.

The first step in this direction has been made by L3-Collaboration which presented the spectra of reconstructed neutral pions 23 . The $\pi^{0}$ distribution proved to be softer when compared to all charged hadrons. In the same time the recent OPAL data on $K^{0}$ production 27 demonstrated stiffening of the massive particle distribution. Numerically

$$
\begin{gathered}
\left(\ln \frac{1}{x_{\max }}\right)_{\pi^{0}}=4.11 \pm 0.18 \quad(\mathrm{~L} 3) ; \\
\left(\ln \frac{1}{x_{\max }}\right)_{c h}=3.603 \pm 0.013 \quad(\mathrm{OPAL}), \\
\left(\ln \frac{1}{x_{\max }}\right)_{c h}=3.71 \pm 0.05 \quad(\mathrm{~L} 3) ; \\
\left(\ln \frac{1}{x_{\max }}\right)_{K^{0}}=2.91 \pm 0.04 \quad(\mathrm{OPAL}) .
\end{gathered}
$$

In accordance with theoretical expectations 316 the value of $\Lambda$ for a pure sample of light hadrons (which is much closer to the "true" scale parameter 
determining the value of running $\alpha_{s}$ ) appeared to be essentially smaller than the "mixed" effective quantity extracted from the charged particle data.

Future increase of statistics for identified particles will make it possible to check the basic LPHD conjecture on the energy independence of the conversion coefficients partons $\rightarrow$ hadrons in the soft confinement regime and for the first time will open up the problem of the comparative study of sea-quarks and gluons contributions to hadroproduction in QCD cascades. 


\section{Bibliography}

1. A. Erdélyi and others. Higher Transcendental Functions, volume 1. McGRAW-HILL, 1953.

2. Yu.L. Dokshitzer and S.I. Troyan. Proceedings of the XIX Winter School of the LNPI, volume 1, page 144. Leningrad, 1984.

3. Ya.I. Azimov, Yu.L. Dokshitzer, V.A. Khoze and S.I. Troyan. Z. Phys., C27:65, 1985.

4. D. Amati and G. Veneziano. Phys. Lett., 83B:87, 1979.

G. Marchesini, L. Trentadue and G. Veneziano. Nucl. Phys., B181:335, 1981.

5. A. Erdélyi and others. Higher Transcendental Functions, volume 2. McGRAW-HILL, 1953.

6. Ya.I. Azimov, Yu.L. Dokshitzer, V.A. Khoze and S.I. Troyan. Z. Phys., C31:213, 1986.

7. G. Cohen-Tannoudji and W. Ochs. Z. Phys., C39:513, 1988.

8. L. van Hove and A. Giovannini. Acta Phys. Pol., B19:917 and 931, 1988.

9. M. Garetto, A. Giovannini, T. Sjöstrand and L. van Hove. In R.C. Hwa, G. Pancheri and Y. Srivastava, editors, Proceedings of Perugia Workshop on Multiparticle Production, page 181. World Scientific, Singapore, 1989.

10. B. Andersson, P. Dahlquist and G. Gustafson. Z. Phys., C44:455 and 461, 1989.

11. V.N. Gribov. preprint LU-TP 91-7, 1991.

12. C.P. Fong and B.R. Webber. Phys. Lett., B229:289, 1989. 
13. A. Stuart and J.K. Ord. Kendall's advanced theory of statistics, volume 1. Griffin, 1987.

14. A.H. Mueller. Nucl. Phys., B213:85, 1983. Erratum quoted ibid., B241:141, 1984.

15. E.D. Malaza and B.R. Webber. Phys. Lett., 149B:501, 1984.

16. Yu.L. Dokshitzer, V.A. Khoze, A.H. Mueller and S.I. Troyan. Rev. Mod. Phys., 60:373, 1988.

17. Yu.L. Dokshitzer, V.A. Khoze and S.I. Troyan. Coherence and physics of QCD jets. In A.H. Mueller, editor, Perturbative QCD, page 241. World Scientific, Singapore, 1989.

18. DELPHI Collaboration, P. Aarnio et al. Phys. Lett., B240:271, 1990.

19. ALEPH Collaboration, D. Decamp et al.. Phys. Lett., B234:209, 1990.

20. MARK2 Collaboration, G.S. Abrams et al.. Phys. Rev. Lett., 64:1334, 1990.

21. OPAL Collaboration, M.Z. Akrawy et al.. Phys. Lett., B247:617, 1990.

22. A.H. Mueller and P. Nason. Phys. Lett., 157B:226, 1985.

23. L3 Collaboration, B. Adeva et al.. L3 Preprint \# 025, 1991.

24. TASSO Collaboration, W. Braunschweig et al.. Z. Phys., C47:198, 1990.

25. Yu.L. Dokshitzer, V.A. Khoze and S.I. Troyan. preprint LU-TP 90-12, 1990.

26. Yu.L. Dokshitzer, V.A. Khoze and S.I. Troyan. preprint LU-TP 91-12, 1991.

27. G. Alexander et al. CERN-PPE / 91-86, 1991. 


\section{Chapter 8}

\section{The Physical Picture of Shadowing in Hard Reactions in Nuclei}

\section{Contents}

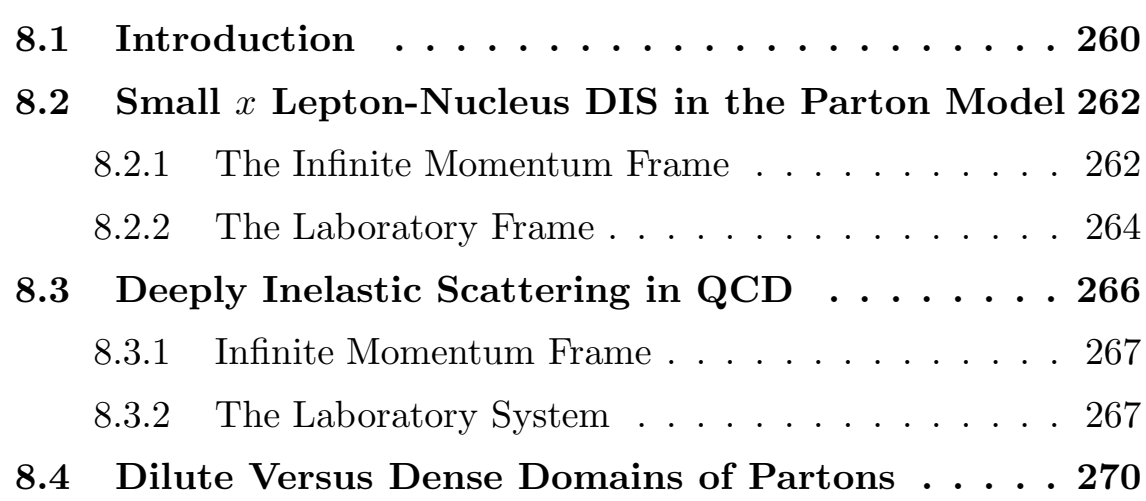


8.5 QCD Phenomenology of Shadowing . . . . . . 271

8.6 Massive $\mu$-Pairs from Hadron-Nucleus Collisions 273

Bibliography . . . . . . . . . . . . . 277

\subsection{Introduction}

Since the advent of the parton model there has been considerable theoretical interest in the question of shadowing in hard processes on nuclei 1. Most interest has centered on the question of shadowing at small values of $x$ in deeply inelastic lepton scattering on nuclei. The first experiment to show definite signs of shadowing, at $Q^{2}$ values in the $\mathrm{GeV}^{2}$ range, was that of Goodman et al. 22. Their results have recently been confirmed in a much more precise experiment by EMC 314. Since the main object here is to explain, qualitatively, how shadowing naturally emerges in the parton model and in QCD, let us simply indicate schematically what the experimental situation appears to be (see Fig. 8.11). In order to emphasize the shadowing regime the $x$-range has been shown on a logarithmic scale. The salient features are:

1 . For $x \lesssim 0.1$ shadowing begins to set in.

2. Shadowing sets in earlier ( $x_{c}$ is larger) for larger nuclei.

3 . The $Q^{2}$-dependence of shadowing is very weak.

4. At the smallest $x$-values shadowing of real and virtual photons is about the same. 
Though in what follows we shall claim that shadowing, with a weak $Q^{2}$ dependence, at small $x$ is natural in QCD there is, unfortunately, no fundamental quantitative model for calculating the magnitude of the shadowing correlations. Though the existance, and the slow $Q^{2}$-dependence 5 , if shadowing can be reliably argued in perturbative $\mathrm{QCD}$, the quantitative properties of shadowing require a good non-perturbative model for high energy soft hadronic reactions 6 . So far there is no such model that has made fundamental contact with QCD. Nevertheless, at present we think we have a good semi-quantitative understanding of shadowing in hard reactions on nuclei and it is that understanding which we wish to explain in the sections that follow.

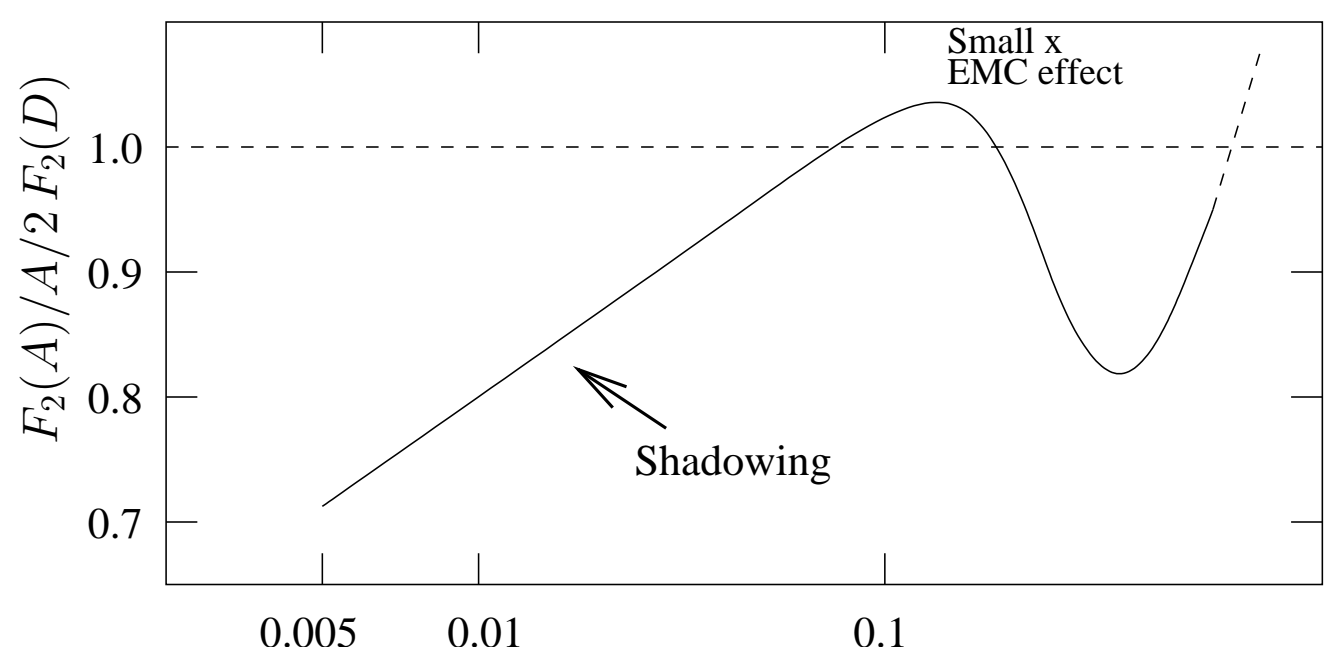

Figure 8.1: A schematic picture of the different regions in DIS. 


\subsection{Deeply Inelastic Lepton Nucleus Scatter- ing at Small $x$ in the Parton Model}

We believe a lot of understanding is obtained by considering inelastic leptonnucleus scattering both in the infinite momentum frame and in the laboratory frame. In the infinite momentum frame one is able to talk of quark and gluon distributions of the nucleus in a natural way, as part of the infinite momentum wavefunction of the nucleus. In the laboratory frame shadowing in inelastic lepton scattering rather closely resembles our picture of shadowing in soft hadronic interactions 7], although here the idea of quark and gluon distributions of the nucleus is not manifest.

\subsubsection{The Infinite Momentum Frame}

In the infinite momentum frame the virtual photon measures the quark distribution in the proton. The situation is illustrated in Fig. 8.2, In this frame the momenta $p$ and $q$ take the form, $p \rightarrow \infty$,

$$
\begin{aligned}
& p \approx\left(p+\frac{m^{2}}{2 p}, 0,0, p\right) \\
& q \approx\left(\frac{Q^{2}}{2 p x}, \overrightarrow{q_{\perp}}, 0\right)
\end{aligned}
$$

with $Q^{2}=q_{\perp}^{2}$ and $x=Q^{2} / 2 p \cdot q$. The virtual photon interacts with the quark of momentum $k \approx x p$ over a time $\tau \approx 2 p x / Q^{2}$ which time is short compared to the normal scale of interaction of the line $k$ which is $\tau_{k} \approx 2 p x / \mu^{2}$ with $\mu$ a soft hadron scale. Thus the photon makes an "instantaneous" measurement of the quark distribution in the infinite momentum wavefunction of the nucleus. 


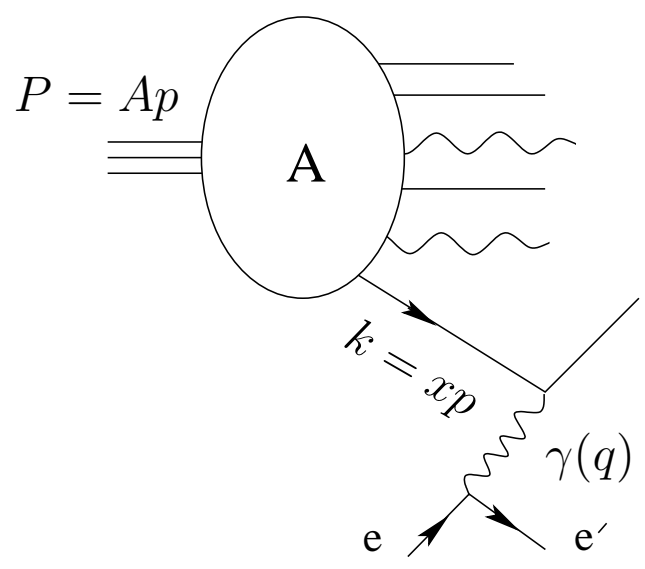

Figure 8.2: The parton model contribution to deep inelastic scattering.

Where are the quarks and gluons located in the wavefunction of a fast nucleus? The valence quarks must be found in a normally contracted nucleus. Thus

$$
(\Delta z)_{\text {valence }} \approx 2 R \frac{m}{p}
$$

is the longitudinal spread of the valence quarks while the sea quarks and gluons have a longitudinal spread determined by the uncertainty relation

$$
(\Delta z)_{\text {sea, glue }} \approx \frac{1}{k_{z}}=\frac{1}{x} p
$$

Let $R$ be the nuclear radius and $R_{i}$ the intranuclear spacing between nucleons in the nucleus. Suppose that $R_{i}(m / p) \lesssim 1 / x p$ or, equivalently, $x \lesssim 1 /\left(R_{i} m\right)$ for a gluon or sea quark of momentum $x p$. Then this gluon, or sea quark, overlaps at least two neighboring nucleons, in the longitudinal direction. At this value of $x$ the spatial gluon and sea quark densities are larger than in 
a proton because the gluons and sea quarks from nucleons which neighbor longitudinally occupy the same physical space. As $x$ further decreases this amount of partonic overlap increases until $x \approx 1 /(2 R m) \sim 0.01-0.02$ for a large nucleus. Here gluon and sea quark overlap is complete.

Thus at small $x$

$$
\frac{d n_{\text {glue, sea }}}{d \text { (area) }} \sim A^{1 / 3}
$$

in the approximation where the sea quarks and gluons from each nucleon in the nucleus are added independently. Equation (8.2) follows directly from the fact that at $x \lesssim 1 /(2 R m)$ there is only one layer, longitudinally, of quarks and gluons. In the naive parton model all partons have $\Delta x_{\perp} \sim 1 \mathrm{fm}$ since all transverse momenta are cut off. Thus the partons actually must reach a density proportional to $A^{1 / 3}$ at small $x$. When quarks and gluons overlap it is natural that they interact and in particular that quarks and antiquarks will annihilate while gluons will be absorbed by quarks and other gluons when the density becomes large. In fact as $A \rightarrow \infty$ we might expect $\mathrm{dn} / \mathrm{d}$ area $) \sim$ constant rather than the $A^{1 / 3}$ growth indicated in (8.2) from the additive approximation. The limiting of the maximum density of partons in a wavefunction is called parton saturation 8 . Any such limitation in parton density due to annihilation and absorption of quarks anf gluons will lead to a smaller DIS cross section; that is, parton saturation is how shadowing appears in the infinite momentum frame.

\subsubsection{The Laboratory Frame}

In the laboratory frame small- $x$ DIS appears as the scattering of a quarkantiquark pair, created by the virtual photon, on the nucleus 7 . The situation 
is here illustrated in Fig. 8.3 with the kinematics given by

$$
\begin{aligned}
p & =(m, 0,0,0) \\
q & =(q-m x, 0,0, q) .
\end{aligned}
$$

The maximum lifetime of the quark-antiquark pair is the same as the lifetime of the virtual photon and is given by

$$
\tau_{\gamma}=\frac{1}{q-(q-m x)}=\frac{1}{m x} .
$$

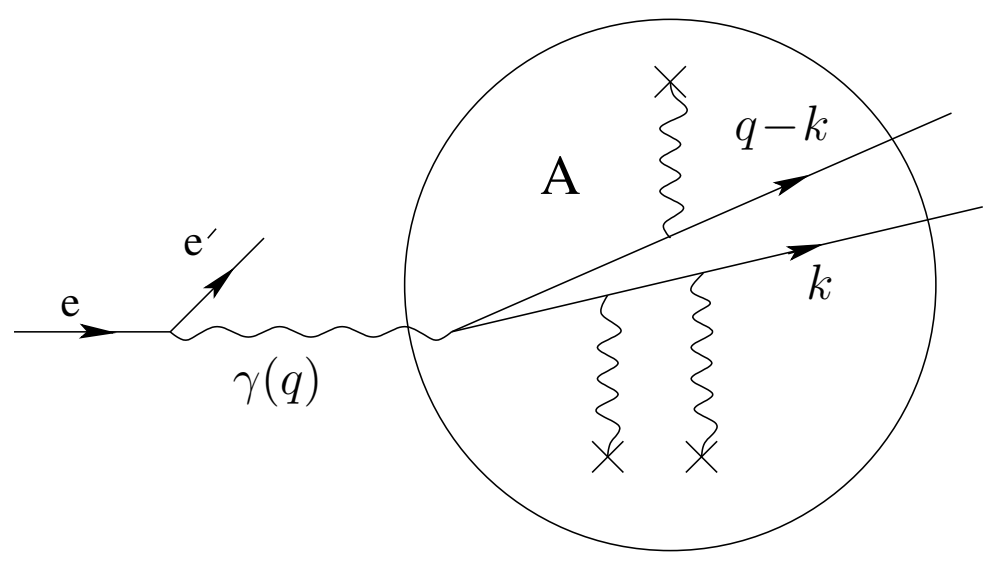

Figure 8.3: Shadowing corrections as viewed in the laboratory frame of the nucleous.

Thus the pair lives over an intranuclear spacing when $1 /(m x) \gtrsim R_{i}$ and the pair lives over the whole nucleus when $1 /(m x) \gtrsim 2 R$. These are the same values of $x$ found in the discussion below (8.1). 
Now in the parton model the pair must have a transverse separation $\Delta x_{\perp} \sim 1 \mathrm{fm}$ since transverse momenta are cut off. Thus the pair should have a cross section with the nucleus on the same order of $\pi \mathrm{fm}^{2}$. If $x$ is large the pair is created in the nucleus and lives only over a single nucleon. Here one expects additivity to be a good approximation, except of course for nuclear physics modifications leading to the ordinary large- $x$ EMC effect. However, when $x \lesssim 1 /\left(R_{i} m\right)$ the pair lives over several nucleons. Because the pair has a cross section about that of normal hadrons one expects to see the start of shadowing. As $x$ is further decreased the lifetime of the quark-antiquark pair increases, the pair lives over more and more nucleons, and so the amount of shadowing increases up to the point where $x \approx 1 /(2 R m)$ at which point shadowing reaches its maximum. Decreasing $x$ below $1 /(2 R m)$ leads to no further significant increase in shadowing in the parton model.

\subsection{Deeply Inelastic Scattering in QCD}

The essential difference between the parton model and QCD 119 is that in QCD transverse momenta are not limited. Thus the transverse size of a quark or gluon can vary from a maximum value of about $1 \mathrm{fm}$ to an arbitrarily small value. This potentially has a significant effect on the arguments given in subsections 8.2.1 and 8.2.2. In 8.2.1 we used the fact that quark and gluon transverse sizes were about $1 \mathrm{fm}$ to argue that significant spatial overlap of partons must occur when $x$ is small. This is not so obvious in QCD since the partons might now have a small enough transverse size to keep their overlap small. In 8.2.2 we argued that the pair cross section with the nucleus was large since the pair separation was on the order of $1 \mathrm{fm}$. Now this also is not so obvious since the pair separation ranges from $1 / Q$ to $1 \mathrm{fm}$ in $\mathrm{QCD}$. 


\subsubsection{Infinite Momentum Frame}

Refer again to Fig. 8.2 where the process is illustrated. Now the $d^{2} k_{\perp}$ integration is free (no constraint from the photonic probe) so long as $k_{\perp}^{2} \lesssim Q^{2}$. This means that all struck quark transverse sizes from $1 / Q$ to $1 \mathrm{fm}$ are important. In the leading logarithmic approximation we may say that

$$
1 / Q \ll \Delta x_{\perp} \ll 1 \mathrm{fm}
$$

with $\Delta x_{\perp}$ the transverse spread of the struck quark. Of course saturation occurs earlier, at larger $x$, for large transverse size quarks than for small transverse size quarks so when $x \lesssim 1 /(2 R m)$ saturation will no longer be complete. In fact the situation is even more complicated. Even if the transvers momentum of the struck quark is large, and so the struck quark has a small spread in $x_{\perp}$, it may be that a gluon, or quark, from which the struck quark evolved had a small transverse momentum and so suffered saturation. For example, in the graph in Fig. 8.4 if $k_{\perp}$ is large but $k_{\perp}^{\prime}$ is small it will be the gluon, $k^{\prime}$, which will overlap with many other quarks and gluons and so have a good chance to produce $k$. Thus in QCD one must follow the whole history of the evolution which leads to the struck quark. This will be discussed further in Sections 8.4 and 8.5 below.

\subsubsection{The Laboratory System}

Refer again to Fig. 8.3. Now, using the same kinematics as given in (8.3), the $\Delta x_{\perp}$ of the quark-antiquark pair depends on $k_{\perp}$. The energy denominator which governs the quark-antiquark fluctuations in the virtual photon is

$$
D=\frac{1}{E_{k}+E_{q-k}-q_{0}},
$$




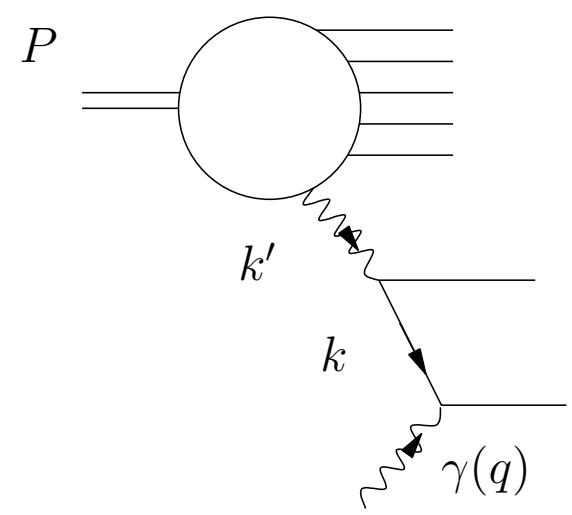

Figure 8.4: DIS in the infinite momentum frame of $P$.

with $E_{k}$ and $E_{q-k}$ being the energies of the quark and antiquark and where $q_{0}$ is given in (8.3b). One easily finds

$$
D \approx\left[m x+\frac{k_{\perp}^{2} q}{2 k(q-k)}\right]^{-1} .
$$

This means that the dominant contribution comes when the slowest of the pair, the fermion having momentum $k$, say, has longitudinal momentum

$$
k \approx \frac{k_{\perp}^{2}}{2 m x} .
$$

That is the longitudinal momentum of the slower member of the pair is coupled to the transverse momentum. The cross section for the pair to interact with the nucleus is proportional to the area occupied by the pair, and thus is proportional to $1 / k_{\perp}^{2}$. The phase space for the pair is proportional to $d k$. 
(Recal that for a given $k$ the value of $k_{\perp}$ is fixed by (8.6)). Thus the contribution of the elements of phase space $d k$ to the cross section is proportional to

$$
\alpha_{s}\left(k_{\perp}^{2}\right) \frac{d k}{k_{\perp}^{2}} \sim \alpha_{s}\left(k_{\perp}^{2}\right) \frac{d k_{\perp}^{2}}{k_{\perp}^{2}} .
$$

There are several points to be made here.

1. Equal regions of $d \ln k_{\perp}^{2}$ contribute equally, except for the slow variation given by $\alpha_{s}\left(k_{\perp}^{2}\right)$.

2. Since large- $k_{\perp}$ regions have a small cross section one might expect the large- $k_{\perp}$ component not to exhibit shadowing while the small- $k_{\perp}$ component should exhibit strong shadowing just as in the parton model.

3. However, the situation is a little more complicated than the sharp distinction drawn between high- $k_{\perp}$ and low- $k_{\perp}$ components given in 2. In fact the high- $k_{\perp}$ component may evolve by emitting an additional gluon, say, as illustrated in Fig. 8.5. If the gluon has a small transverse momentum than the quark-antiquark-gluon system will interact strongly with the nucleus even if the quark-antiquark pair occupy a small area.

Thus we have reached the same point about the necessity of considering evolution as we reached in Sec 8.3.1 a little earlier. We shall return in Sec 8.5 to describe a QCD calculation which, at least partially, takes this evolution into account, but first we would briefly like to describe the idea of parton saturation 8 a little more. 


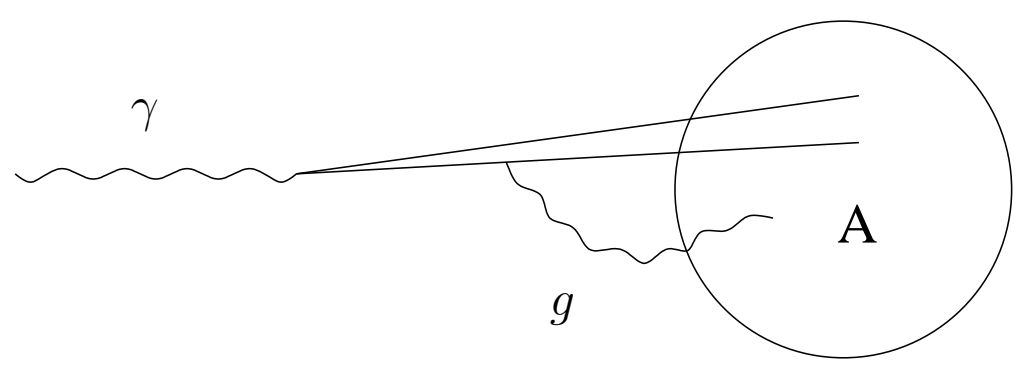

Figure 8.5: Shadowing due to presence of additional gluon emission.

\subsection{Dilute Versus Dense Domains of Partons}

The usual DGLAP equation of Chapter 1 at small- $x$ takes the form

$$
Q^{2} \frac{\partial}{\partial Q^{2}} x G_{A}\left(x, Q^{2}\right)=\frac{\alpha_{s}\left(Q^{2}\right)}{\pi} C_{A} \int_{x} \frac{d x^{\prime}}{x^{\prime}} x^{\prime} G_{A}\left(x^{\prime}, Q^{2}\right)
$$

written alone for the gluon distribution in the nucleus $A$. This equation gives a rapidly growing gluon distribution as $x$ becomes small. When $x G_{A}$ becomes large enough there are gluon recombination terms which become important. When corrections to (8.7) become important it is a signal that a dense system of partons rather than the dilute system described by (8.7) is being reached. The first corrections to (8.7), in the small- $x$ limit, are known and lead to a modified evolution which reads 10

$$
\begin{aligned}
Q^{2} \frac{\partial}{\partial Q^{2}} & x G_{A}\left(x, Q^{2}\right)=\frac{\alpha_{s} C_{A}}{\pi} \int_{x} \frac{d x^{\prime}}{x^{\prime}} x^{\prime} G_{A}\left(x^{\prime}, Q^{2}\right) \\
& -\left(\frac{\alpha_{s} C_{A}}{\pi}\right)^{2} \frac{\pi^{3}}{\frac{16}{9} \pi R^{2} Q^{2}} \int_{x} \frac{d x^{\prime}}{x^{\prime}}\left[x^{\prime} G_{A}\left(x^{\prime}, Q^{2}\right)\right]^{2}
\end{aligned}
$$


This equation is really valid only when the correction term (the second term on the right-hand side), is small compared to the usual evolution term. When the correction term is comparable to the usual evolution term, the first term on the right-hand side of (8.8), in fact there are other important corrections whose form is not known at present. Thus we really only have a theory for the non-linear terms in the evolution in the low density regime. Nevertheless (8.8) is an interesting equation in that the second term stabilizes the growth of normal evolution and leads to a limiting value for $x G\left(x, Q^{2}\right)$ as $x \rightarrow 0$. We can see roughly where this saturation sets in by finding where the right-hand side of (8.8) loses its $x$-dependence. This clearly occurs when

$$
x G_{A}\left(x, Q^{2}\right)=\frac{16 R^{2} Q^{2}}{9 \alpha_{s} C_{A}} .
$$

Thus if (8.8) is not too misleading we expect the number of gluons per unit area $d n_{g} / d($ area $)$ to saturate at a value roughly given by

$$
\frac{d n_{g}}{d(\text { area })}=\frac{x G_{A}\left(x ; Q^{2}\right)}{\pi R^{2}} \approx \frac{16}{9 \pi \alpha_{s} C_{A}} Q^{2},
$$

a value independent of $A$, in contrast to the additive result given by (8.2). It must be emphasized, however, that (8.8) is not really valid in the high density limit and so (8.9) and (8.10) should be taken with some caution. One of the very important open theoretical problems is to obtain a formalism which correctly handles, at least qualitatively, the dense parton regime 11 .

\subsection{QCD Phenomenology of Shadowing}

One cannot make absolute predictions for structure functions in QCD. The DGLAP equation can only be solved once an initial distribution is given. That 
is if, for example, $x G\left(x, Q_{0}^{2}\right)$ is given, for all values of $x$ at a given $Q_{0}^{2}$, then $x G\left(x, Q^{2}\right)$ may be calculated by (8.7) or (8.8). The situation is illustrated in Fig. 8.6.

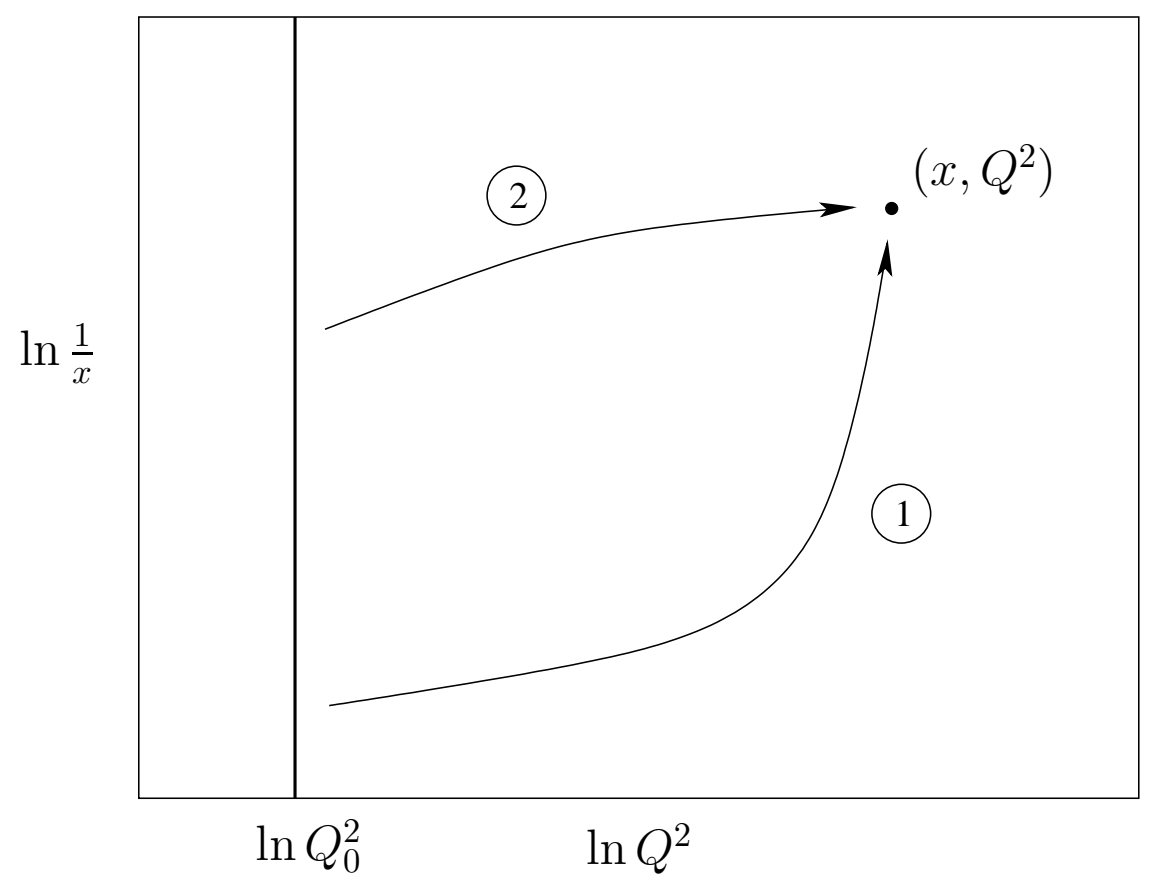

Figure 8.6: Different ways of reaching the point $\left(x, Q^{2}\right)$ from the initial line $\ln Q^{2}=\ln Q_{0}^{2}$.

To evaluate, say, $x G\left(x, Q^{2}\right)$ at a particular point $\left(x, Q^{2}\right)$ we must integrate (8.8) starting from an initial distribution at $Q_{0}^{2}$. Since (8.8) is only valid away from the dense regime $Q_{0}^{2}$ must be chosen to obey $Q_{0}^{2} \gtrsim 2 \mathrm{GeV}$. Trajectories 1 and 2 in Fig. 8.6 indicate schematically examples of different regions of 
integration necessary in order to go from the initial distribution to, say, $x G$ evaluated at $\left(x, Q^{2}\right)$. Trajectory "1" shows evolution proceeding to large values of $Q^{2}$ while $x$ is still large. From our previous discussions we expect ordinary evolution, (8.7), to be valid here and the contribution of " 1 " is a contribution which should exhibit no shadowing. Trajectory "2" starts from small values of $x$ when $Q^{2}$ is still small. Thus we expect the second term on the right-hand side of (8.8) to be important here and thus shadowing should occur. However, even more important for " 2 " is the value assigned to the parton distributions at $Q_{0}^{2}$. One must decide whether to put in shadowed or non-shadowed initial distributions. This must be done from the data 115 . There is no reliable way to get initial distributions from QCD theory alone.

What then does QCD have to say about shadowing in deeply inelastic lepton scattering? Qiu 5 has done detailed calculations taking initial distributions which exhibit shadowing and are partially constrained by the data. $\mathrm{He}$ finds that if parton distributions exhibit significant shadowing at $Q_{0}^{2}=4 \mathrm{GeV}$ then for fixed small- $x$ shadowing goes away very slowly as one increases $Q^{2}$ even up to values of $Q^{2} \gtrsim 100 \mathrm{GeV}^{2}$. This is, we think, a solid prediction of perturbative QCD and is a result not widely believed before the recent EMC data. Of course if one decreases $x$ as $Q^{2}$ increases QCD predicts that shadowing never goes away no matter how large $Q^{2}$ might be.

\subsection{Large Mass $\mu$-Pair Production in Hadron-Nucleus Collisions}

QCD factorization allows one to relate large mass $\mu$-pair production to deeply inelastic lepton-hadron scattering. Consider the process of proton $+A \rightarrow$ $\mu^{+} \mu^{-}(Q)+$ anything illustrated in Fig. 8.7 


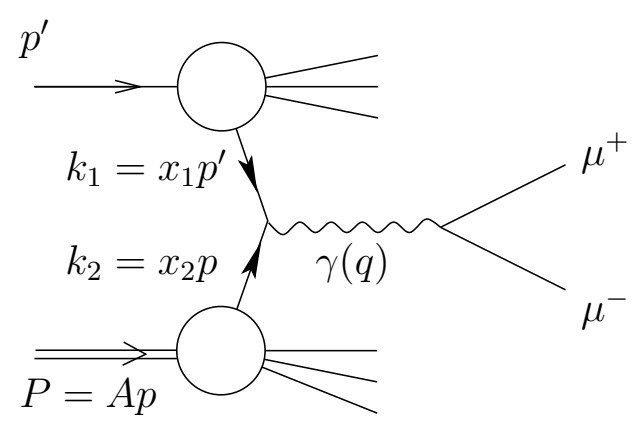

Figure 8.7: The kinematics of $\mu$-pair production.

The cross section is given, in leading logarithmic approximation, by

$$
\begin{gathered}
\frac{d \sigma}{d Q^{2} d y}=\frac{8 \pi \alpha_{s}^{2}}{9\left(Q^{2}\right)^{2}} \sum e_{a}^{2} \\
\cdot\left[x_{1} P^{a}\left(x_{1}, Q^{2}\right) x_{2} P_{A}^{\bar{a}}\left(x_{2}, Q^{2}\right)+x_{1} P^{\bar{a}}\left(x_{1}, Q^{2}\right) x_{2} P_{A}^{a}\left(x_{2}, Q^{2}\right)\right],
\end{gathered}
$$

where $a$ indicates the quark flavor. $P^{a}\left(x, Q^{2}\right)$ is the distribution of quarks of flavor $a$ in the proton while $P_{A}^{a}\left(x, Q^{2}\right)$ is the distribution of quarks of flavor $a$ in the nucleus. These distributions should be the same as those observed in deeply inelastic lepton-proton and lepton-nucleus reactions. In particular at small values of $x_{2}$ we should see the same amount of shadowing here as found by EMC at comparable $x$ and $Q^{2}$ values. An experiment measuring this small- $x_{2}$ region has recently been completed at Fermilab 12 and the results appear consistent with those obtained from DIS 13 .

Though (8.11) is a correct prediction of QCD factorization it does require high $Q^{2}$ in order to be valid. How large must $Q^{2}$ be in order that (8.11) be valid? Consider the schematic illustration of the process in Fig. 8.8. 


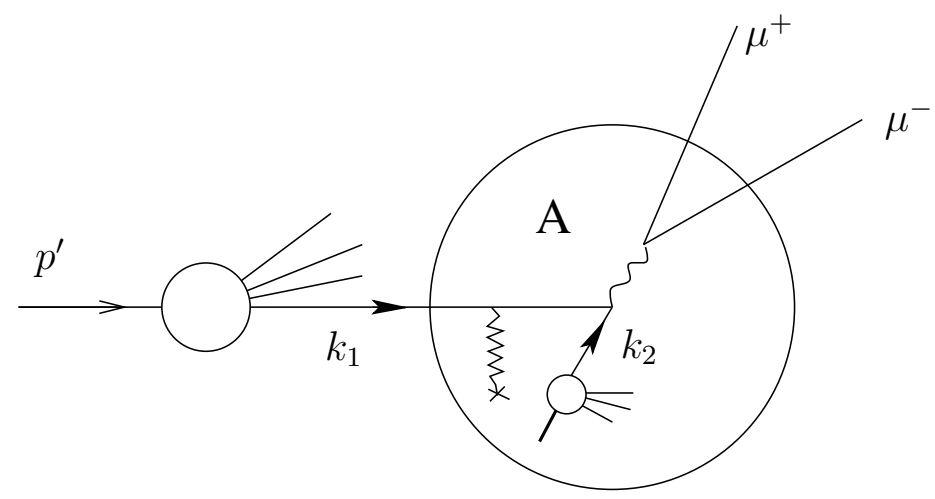

Figure 8.8: Initial state scattering in $\mu$-pair production on a nucleus $A$.

As the line $k_{1}$ passes through the nucleus it may interact with nucleons in the nucleus. After much study we know that such interactions will not spoil factorization at large $Q^{2}$, but it is also known that such interactions will change the $q_{\perp}^{2}$ distribution of the $\mu$-pair. In the present circumstance we are integrating over the $q_{\perp}$ distribution so this does not matter, however, $x_{1}$ is being held fixed so we must require that the longitudinal energy loss of the $k_{1}$-line be much less than its value. That is, we must require

$$
k_{1}=x_{1} p^{\prime} \gg \mu \cdot \# \text { of scatterings of } k_{1}
$$

since each soft scattering can stimulate an energy loss, $\mu$. Clearly the number of scatterings may grow like $R$, however, it is difficult to estimate the coefficient of that growth, just as it is difficult to estimate the value of $\mu$ in (8.12). Let us say

$$
x_{1} p^{\prime} \gg \mu \cdot(\mu R)
$$

with $\mu$ some soft hadronic scale, perhaps $300 \mathrm{MeV}$ or so. (This is just a guess 
for $\mu$ !) Now

$$
2 x_{1} p^{\prime} m x_{2}=Q^{2}
$$

so combining (8.13) and (8.14) one arrives at

$$
Q^{2} / \mu^{2} \gg 2 R m x_{2}
$$

as the condition for factorization to apply. If $R \approx 7 \mathrm{fm}$ and we want factorization to apply over a wide range of $x_{2}$ then $Q \gg 6 \mu$, which means that $Q$ must be greater than a few $\mathrm{GeV}$, at least for a large nucleus. 


\section{Bibliography}

1. For a review see L. Frankfurt and M. Strikman. Phys. Rep., 160:235, 1988.

2. M.S. Goodman et al.. Phys. Rev. Lett., 47:293, 1981.

3. J. Ashman et al.. Phys. Lett., 202B:603, 1988.

4. M. Arneodo et al.. Phys. Lett., 211B:493, 1988.

5. J. Qiu. Nucl. Phys., B291:746, 1987.

6. L. Frankfurt and M. Strikman. Nucl. Phys., B316:340, 1988.

7. J.D. Bjorken and J. Kogut. Phys. Rev., 28:1341, 1973.

8. L.V. Gribov, E.M. Levin and M.G. Ryskin. Phys. Rep., 100:1, 1983.

9. A.H. Mueller. In Proceedings of the $17^{\text {th }}$ Recontre de Moriond, ed. J.Tran Thanh Van, Editions Frontieres, Gif-sur-Yvette, 1982.

10. A.H. Mueller and J. Qiu. Nucl. Phys., B268:427, 1986.

11. for attempt in this direction see A.H. Mueller. Nucl. Phys., B307:34, 1988; B317:573, 1989; B335:115, 1990.

12. D.M. Alde et al.. Phys. Rev. Lett., 64:2479, 1990.

13. L. Frankfurt, M. Strikman and S. Liuti. Phys. Rev. Lett., to be published. 


\section{Chapter 9}

\section{Radiophysics of QCD Jets}

\section{Contents}

9.1 On Structure of Particle Flows in Multijet Events 281

9.2 QCD Portrait of an "Individual Jet" . . . . . . . 284

9.2.1 Collimation of Energy in Jet . . . . . . . . . 285

9.2.2 Energv Spectrum Within a Given Cone . . . . . . 289

9.2 .3 Collimation of Multiplicity Inside Jet . . . . . . . 293

9.3 Multiplicitv Flow Pattern for $q \bar{q} q$ Ensemble . . . 294

9.3.1 Radiation Pattern for $q \bar{q} \gamma$-events . . . . . . . . . 296

9.3.2 Radiation Pattern for $q \bar{q} g$-events . . . . . . . . . . 299

9.3.3 Average Multiplicity of 3-iet events . . . . . . . . . 301

9.4 QCD Drag Effect in Interiet Particle Flows . . . . 306

Bibliography . . . . . . . . . . . . . 318 
The source of multiple hadroproduction in hard processes is gluon bremsstrahlung, so one should expect that in the framework of LPHD the observed jets of hadrons are a consequence of the color dynamics at small distances. Therefore, the detailed features of the parton shower system, such as the flow of color quantum numbers, influence significantly the distribution of color singlet hadrons in the final state. See Refs. [1].

As we discuss below, the collimation of the QCD cascade around the parent parton becomes stronger as the parton energy increases. If one keeps the angle between the two jets fixed, then with increase of the total energy these jets become more and more distinguishable experimentally. Moreover, the collimation of an energy flux grows much more rapidly as compared to a multiplicity flow. Shrinkage of the characteristic opening angle permits one to introduce the notation of the "intRAjet" and the "intERjet" hadron flows. Therefore, at asymptotically high energies each event should possess the clear geometry, that reflects the topology of the final hadronic system in terms of partons which participated directly in the hard interaction. The inclusive space-energy portrait of events represents a natural partonometer for registration of the kinematics of the energetic parent partons. While the hard component of a hadron system (a few hadrons with energy fraction $z \sim 1$ ) determines the partonic skeleton of an event, the soft component (hadrons with $z \ll 1$ ) forms the bulk of multiplicity.

Closely following the radiation pattern, associated with the partonic skeleton, the soft component is concentrated inside the bremsstrahlung cones of QCD jets. Theoretically, the opening angle of each cone $\Theta_{0}$ is bounded by the nearest other jet, since at larger angles $\Theta>\Theta_{0}$ particles are emitted coherently by the overall color charge of both jets. As the result, the total 
multiplicity is given by the additive sum of the contributions of the bounded individual jets.

In this Chapter we discuss mainly the QCD portrait of jet ensembles in hard processes. The emphasis is made on the collective QCD phenomena in jet dynamics. Such a phenomenon has been first observed in experiments (see Refs. 447) studying the angular flows of hadrons in three-jet $(q \bar{q} g)$ events from $e^{+} e^{-}$annihilation, the so-called string ${ }^{[8]}$ or drag 9 effect. The $\mathrm{PEP} / \mathrm{PETRA}$ data have strongly supported the predicted drag of the interjet particles in the direction of the gluon jet (net destructive interference in the region between the $q$ and $\bar{q}$ ), for details see Section 9.4. Drag effect studies are also successfully performed at LEP 1011.

The data demonstrate that wide-angle particles really do not belong to any particular jet, but have emission properties dependent on the overall jet ensemble. Surely, it is entirely unremarkable that the quantum mechanical interference effects should be observed in QCD. Of real importance is that the experiment demonstrates that these effects survive the hadronization phase.

Detailed studies of string-like phenomena are of importance for the high energy reactions. These effects are interesting not only in their own right as test of QCD. They could be valuable in helping to dig out the possible new physics signals from the conventional QCD backgrounds.

\subsection{On Structure of Particle Flows in Multijet Events}

Traditionally, the final state structure in a hard collision is interpreted in terms of a certain number of hadron jets having specific angles, energies, masses etc. This has been a very fruitful procedure especially as regards 
three jet events in $e^{+} e^{-}$collisions, where the gluon was found, and high$p_{\perp}$ jet events in hadron collisions, where the point-like nature of quark and gluon interactions is measured. However, the separation of an event into a certain number of jets is inherently ambiguous, especially as one goes to higher energies. The ambiguity comes from several sources.

1. A QCD jet exhibits fractal structure, consisting of a number of sub-jets, which makes the jet definition highly artificial.

2. Interjet hadrons which form a sizable part of the total event multiplicity are distributed according to the color properties of the event as a whole and, as a matter of principle, cannot be associated with any particular jet.

Attempting to force particles to belong to some jet in an event may cause some difficulties. If the jet algorithms do not use infrared safe quantities, comparison with QCD cannot be carried to higher orders and the whole procedure, though adequate when only crude data and crude calculations are available, may have limited quantitative significance. If the jet finding algorithms are infrared stable, the procedure for assigning particles to jets is in principle all right, but, as higher energy events become more complicated this procedure may simply not be efficient.

As higher energies are attained the purely inclusive and calorimetric characteristics for quantitatively dealing with hard collisions become preferable to organizing the event according to a certain number of jets 12 18. There is in general a rather direct correspondence between the jet directions and energy flux directions, so that one may naturally study the jet shapes and any other characteristics of the hadronic system by introducing inclusive correlations among energy fluxes and multiplicity flows 216. In this case one does not need to apply to event selection procedures or jet finding algorithms. The 
calorimetric quantities are free from soft and collinear singularities, and are therefore well controlled perturbatively.

As the simplest example consider the angular distribution of the multiplicity flow in two-jet events of $e^{+} e^{-}$annihilation. Its study is accessible through an (energy $)^{2}-$ multiplicity correlation $\left(E^{2} M C\right)$

$$
\begin{array}{r}
\frac{d N}{d \Omega_{\vec{n}}}=\sum_{a, b} \int d E_{a} d E_{b} d E_{\vec{n}} \frac{E_{a} E_{b} d \sigma_{3}}{\sigma_{2} d E_{a} d E_{b} d E_{\vec{n}} d \Omega_{a} d \Omega_{b} d \Omega_{\vec{n}}}, \\
\sigma_{2}=\sum_{a, b} \int d E_{a} d E_{b} \frac{E_{a} E_{b} d \sigma_{2}}{d E_{a} d E_{b} d \Omega_{a} d \Omega_{b}}, \quad \vec{n}_{b} \approx-\vec{n}_{a},
\end{array}
$$

where the sum goes over all particle types. The energy weighted integrals over $E_{a}$ and $E_{b}$, at fixed angular directions $\vec{n}_{a}$ and $\vec{n}_{b} \approx-\vec{n}_{a}$, specify the "jet directions" about which one has an associated multiplicity distribution at variable angular direction $\vec{n}(\Omega)$. The cross section (9.1b), describing the correlation between two back-to-back energy fluxes (EEC), contains the known double logarithmic form factor (see Chapter 1 and Refs. [1819) that reflects the natural disbalance of the jet direction, caused by gluon bremsstrahlung.

The same angular distribution may be discussed in terms of a more simple double-inclusive correlation between the energy flux and the multiplicity flow (EMC)

$$
\begin{gathered}
\frac{d N_{2}}{d \Omega_{\vec{n}}}=\sum_{a} \int d E_{a} d E_{\vec{n}} \frac{E_{a} d \sigma_{2}}{\sigma_{1} d E_{a} d E_{\vec{n}} d \Omega_{a} d \Omega_{\vec{n}}}, \\
\sigma_{1}=\sum_{a} \int d E_{a} \frac{E_{a} d \sigma_{1}}{d E_{a} d \Omega_{a}} .
\end{gathered}
$$

The point is that here the main contribution also comes from the two-jet sample whose kinematics is practically fixed by the choice of the direction 
$\vec{n}_{a}$. The difference between the distributions (9.1) and (9.2) occurs only when the angular direction $\vec{n}$ is taken parametrically close to the backward "jet axis", $\vec{n} \approx-\vec{n}_{a}$. In this case the shape of the distribution (9.2) near $\vec{n}=-\vec{n}_{a}$ becomes somewhat wider due to the natural "shaking" of the nonregistered jet in QCD. A typical "shaking angle" can be estimated 20 as $\Theta_{s h} \sim(\Lambda / W)^{\chi}$, where $\chi=b /\left(b+4 C_{F}\right) \approx 0.64$ for $n_{f}=3$ active flavors.

The drag effect physics becomes accessible through a more complicated example, $E^{3} M C$, see Section 9.4 Studies of the two-particle angular distributions via the $E M^{2} C$ are discussed there.

Another application of this method is the "restricted cone" inclusive particle distribution without the need of any specific jet-finding algorithm (see Subsection (7.8). The distribution of particles which enter into a cone of aperture $\Theta$ around the "jet axis" can be defined in terms of $E M C$ as

$$
\bar{D}^{\Theta}(x, E)=\frac{1}{E} \int_{0}^{\Theta} d \Theta_{12} \int E_{1} d E_{1} \int d E_{2} \frac{d \sigma_{2}}{\sigma d E_{1} d E_{2} d \Theta_{12}} \delta\left(x-\frac{E_{2}}{E}\right) .
$$

In the general case multiple correlations of the " $E^{\alpha} M^{\beta} C$-kind" can be introduced. This means that one should fix $\# \alpha$ jet directions with help of energy fluxes, and then consider correlations between \# $\beta$ multiplicity flows. The procedure of normalizing to a given energy configuration will ensure that results are finite and well-behaved.

\subsection{QCD Portrait of an "Individual Jet"}

Let us consider the general inclusive characteristics which may be called, in some sense, the characteristics of an isolated jet (neglecting the mutual influence of jets in their ensemble). One can study the properties of an 
individual quark jet by measuring the different inclusive distributions in the process $e^{+} e^{-} \rightarrow$ hadrons. The decay into two gluons of the $C$-even heavy quarkonium states, $\chi_{Q}=Q \bar{Q}$, might define, by analogy, the individual gluon jets. The notion of the isolated jet makes sense, of course, if one does not deal with the azimuthal effects but considers only multiplicities, energy spectra and correlations, etc. In this case all the influence of the jet ensemble on a given jet may be encoded in a single parameter $\Theta_{0}$, the jet opening angle. This angle, in essence, is the angle between the considered jet and the nearest other one.

Multiplicity, energy spectra of particles and other characteristics of the QCD partonic cascade prove to depend not on the jet's energy $E$ but on the hardness of the process producing this jet, i.e. on the largest possible transverse momentum of particles inside the jet, $Q=E \Theta_{0}$ at $\Theta_{0} \ll 1$, which corresponds, of course, to the transverse momentum of the jet itself.

\subsubsection{Collimation of Energy in Jet}

Let us consider a single jet with energy $E$ and opening angle $\Theta_{0}$ and try to answer the question, what is the angular size $\Theta_{z}\left(Q_{0} / E \lesssim \Theta_{z} \lesssim \Theta_{0}\right)$ of the cone, where the definite fraction $z \sim 1$ of the jet energy is deposited (see Fig. 9.1). The smaller is the angle, where the bulk of the energy is concentrated (i.e. aperture of the energy flux), the higher is the jet collimation. Experimentally it corresponds to the calorimetric measurement of the energy flux deposited within the given cone.

As we saw above the sequential parton decays in a cascade are ordered in angles (the "hard" decays due to the LLA kinematics and "soft" ones due to the QCD coherence). Therefore a calorimeter measures an energy of a subjet which is initiated by a parton $B$ produced at that stage of the cascade

evolution where the characteristic transverse momenta in the decays, $k_{\perp}$, are 


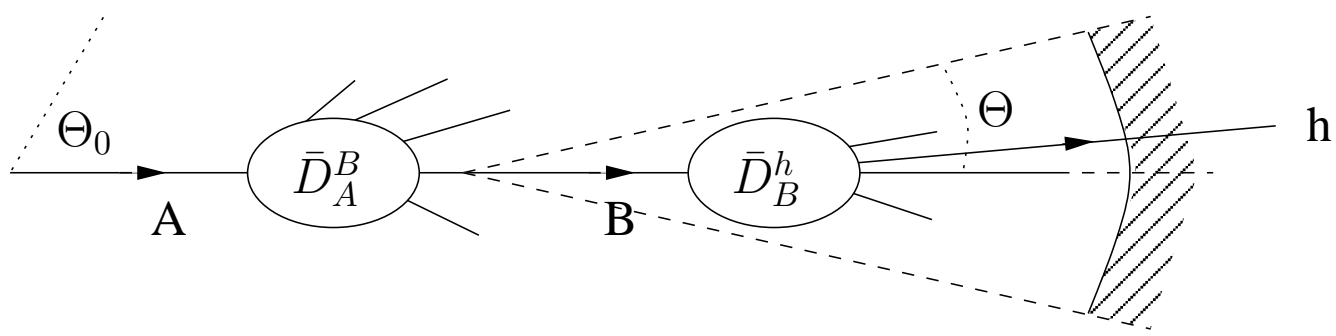

Figure 9.1: Production of a subjet $B$ registered by the calorimeter with the angular aperture $\Theta$.

of order $z E \Theta$. In other words, a calorimeter with an aperture $\Theta$ registers the energy spectrum of the intermediate partons at the certain phase of the development of the partonic system. Hence the probability that the energy fraction $z$ is deposited in a cone with the opening angle $\Theta$ should be related to the inclusive spectrum of partons

$$
\bar{D}_{A}\left(z ; E \Theta_{0}, E \Theta\right)=\sum_{B=q, g} \bar{D}_{A}^{B}\left(z ; E \Theta_{0}, E \Theta\right),
$$

where $A$ denotes the incoming parent parton $(A=q, g)$. We assume here that the type of registered parton $B$ is not identified.

To quantify the energy collimation in a jet let us suppose that the deposited share of energy is large, $z \rightarrow 1$. Then the valence contribution dominates (see Chapter 11)

$$
\bar{D}_{A}^{A}\left(z ; E \Theta_{0}, E \Theta\right) \propto(1-z)^{-1+4 C_{A} \Delta \xi},
$$

where $\Delta \xi=\xi\left(E \Theta_{0}\right)-\xi(E \Theta)$ corresponds to the evolution from the incoming 
parton $A$ to the parton $B=A$, decaying inside a given cone with opening angle $\Theta$.

At a fixed value of $z$ this formula describes the distribution in $\Theta$, that has a characteristic maximum at some angle $\Theta=\Theta_{z}$. Indeed, $\bar{D}_{A}^{A}(z, \Delta \xi) \rightarrow \delta(1-z)$ when $\Theta \rightarrow \Theta_{0}$ and the probability $\bar{D}$ for $z \neq 1$ should rapidly decrease. On the contrary, with $\Theta$ decreasing (down to $\Theta \gtrsim \Lambda / E$ ) the share of emission outside the cone grows. The quantity $\Delta \xi$ increases, and so the probability $\bar{D}$, that the energy fraction $z$ is deposited in the cone, decreases again (an effect similar to the Sudakov form factor suppression).

To illustrate the energy dependence of the quantity $\Theta_{z}$,

$$
\frac{\Theta_{z}}{\Theta_{0}}=\left(\frac{E \Theta_{0}}{\Lambda}\right)^{-\gamma(z)}
$$

we present the approximate values of $\gamma(z)$ at $z=0.9$ and $z=0.5$ (see Fig. (9.2):

$$
\begin{aligned}
& \gamma_{q}(0.9) \approx 0.55, \quad \gamma_{g}(0.9) \approx 0.30 \\
& \gamma_{q}(0.5) \approx 0.83, \quad \gamma_{g}(0.5) \approx 0.54 .
\end{aligned}
$$

As one can see, the energy collimation grows as energy increases, being stronger for a quark jet than for a gluon jet. 


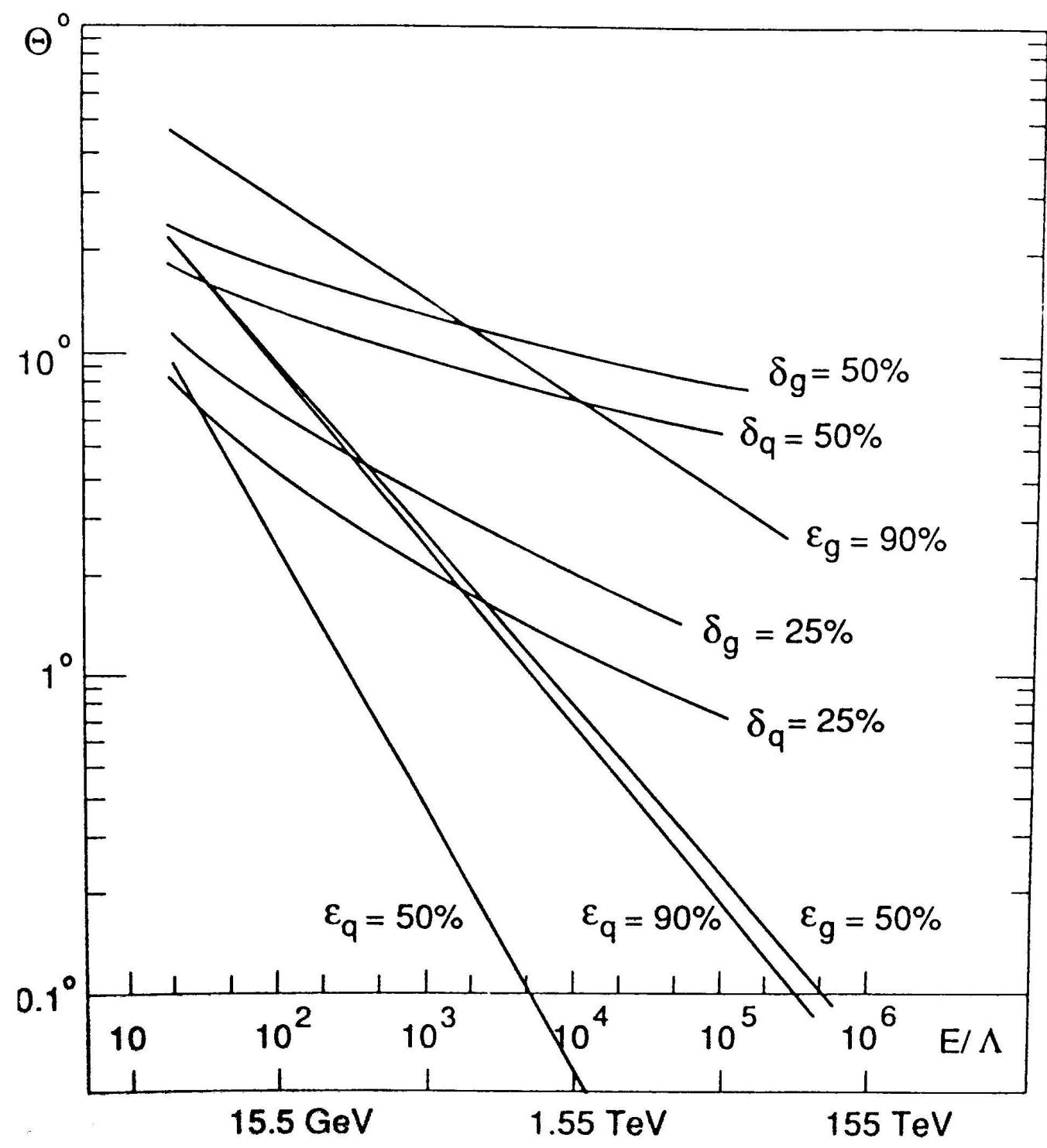

Figure 9.2: Shrinkage of the cones, in which the fixed shares of multiplicity $(\delta)$ and energy $(\epsilon)$ of a jet $A(A=q, g)$ are deposited. 


\subsubsection{Energy Spectrum Within a Given Cone}

More subtle is the spectral characteristic of the energy flux registered by a calorimeter with the angular aperture $\Theta$. Such a quantity represents, strictly speaking, a correlation between the energy flux and one of the particles within it. This is the double inclusive quantity: a parton $B$ is registered together with one of its offspring, a particle $h$, as shown in Fig. 9.1. The distribution over $x$, the energy fraction of the hadron $h$ within the registered energy flux, may be presented as the convolution

$$
F_{A}^{h}\left(x, \Theta ; E, \Theta_{0}\right)=\sum_{B=q, g} \int_{x}^{1} d z \bar{D}_{A}^{B}\left(z ; E \Theta_{0}, z E \Theta\right) \bar{D}_{B}^{h}\left(\frac{x}{z} ; z E \Theta, Q_{0}\right)
$$

Here $\bar{D}_{A}^{B}$ determines the probability to find the parton $B$, initiating the subjet $A$, and $\bar{D}_{B}^{h}$ describes the distribution over the energy fraction $x / z$ of hadron $h$ in a subjet $B$.

Note that one could obtain this result directly by integrating the appropriate standard expression for the double-inclusive correlation between particles (see, e.g., [19] and references therein).

An integration over $z$ in (9.6) corresponds to the simplest case when it is known that the energy flux is deposited within a given solid angle, but the corresponding energy share is not measured. If one fixes the value of $z$, the integration should be omitted. To estimate the integral one can neglect $z$ in the arguments of all $\operatorname{logs}$ since only the values of $z \sim 1$ are essential. This stems from the behavior of the function $\bar{D}_{B}^{h}$ at $x \ll 1$ :

$$
\bar{D}_{B}^{h}\left(x ; E \Theta_{0}, Q_{0}\right)=\frac{1}{x} \cdot \rho\left(\ln \frac{1}{x}, \ln \frac{E \Theta_{0}}{\Lambda}, \ln \frac{Q_{0}}{\Lambda}\right),
$$

where $\rho$ is a slowly changing function that describes the hump-backed plateau (see Chapter 7). 
For a better understanding of the correlative nature of (9.6) one may consider the two limits.

$$
\text { (i) } \quad \Theta \rightarrow \Theta_{0}, \quad \bar{D}_{A}^{B} \rightarrow \delta(1-z) \delta_{A}^{B}, \quad F_{A}^{h} \rightarrow \bar{D}_{A}^{h}\left(x ; E \Theta_{0}, Q_{0}\right) .
$$

In this case the whole energy flux of a jet $A$ is deposited in the calorimeter, and the registered particle spectrum coincides with that in the overall jet.

(ii) $\quad \Theta \rightarrow \frac{Q_{0}}{z E}, \quad \bar{D}_{B}^{h} \rightarrow \delta(1-x / z) \delta_{A}^{h}, \quad F_{A}^{h} \rightarrow x \bar{D}_{A}^{h}\left(x ; E \Theta_{0}, Q_{0}\right)$.

Here a subjet $B$ is reduced to one hadron $h$, the energy flux is predetermined by the value of $x$. The correlation disappears, and the expression (9.6) factorizes into $x$, the energy flux, and $\bar{D}_{A}^{h}$, the probability to find a hadron $h$ with an energy fraction $x$ inside a jet $A$.

The correlation, that in a general case $(x \sim 1)$ is described by (9.6), disappears, in fact, for soft hadrons as well. Emission of such particles proves to be less sensitive to the energy balance. Substituting (9.7) into (9.6) for $x \ll 1$ one obtains

$$
F_{A}^{h}\left(x, \Theta ; E, \Theta_{0}\right) \approx \frac{\langle C\rangle_{A}}{N_{c}} \cdot \bar{D}_{g}^{h}\left(x ; E \Theta, Q_{0}\right)
$$

with $\bar{D}_{g}^{h}$ the inclusive spectrum in a gluon jet with energy $E$ and opening angle $\Theta_{0}$. The quantity $\langle C\rangle_{A}$ here is the average color current of partons caught by the calorimeter:

$$
\langle C\rangle_{A}=\left\langle z^{g}\right\rangle_{A} \cdot N_{c}+\left\langle z^{q}\right\rangle_{A} \cdot C_{F}
$$

where

$$
\left\langle z^{B}\right\rangle_{A}=\int_{0}^{1} d z z \bar{D}_{A}^{B}\left(z ; E \Theta_{0}, E \Theta\right) \quad, \quad\left\langle z^{g}\right\rangle_{A}+\left\langle z^{q}\right\rangle_{A}=1 .
$$


The integral of (9.6) over $x$ gives the multiplicity of hadrons of type $h$ :

$$
N_{A}^{h}\left(\Theta ; E, \Theta_{0}\right)=\frac{\langle C\rangle_{A}}{N_{c}} \cdot N_{g}^{h}(E \Theta) .
$$

Thus, the yield of particles in the registered part of $A$-jet is proportional to that in an isolated gluon jet with hardness $Q=E \Theta$. The hardness of a primary jet, $E \Theta_{0}$, affects only the proportionality coefficient, that is the average color current of the parent parton that produces the registered part of particle flow.

The value of the mean color current of a parton subjet depends on the momentum balance between quarks and gluons in evolution of the jet $A$. The momenta carried away by quarks $\left\langle z^{q}\right\rangle_{A}$ and gluons $\left\langle z^{g}\right\rangle_{A}$ are calculable in the LLA (see Problem 9.11). Substituting them into (9.9) one obtains

$$
\begin{aligned}
\langle C\rangle_{q} & =\langle C\rangle_{\infty}-\alpha\left(N_{c}-C_{F}\right)\left(\frac{\ln (E \Theta / \Lambda)}{\ln \left(E \Theta_{0} / \Lambda\right)}\right)^{\gamma}, \\
\langle C\rangle_{g} & =\langle C\rangle_{\infty}+\beta\left(N_{c}-C_{F}\right)\left(\frac{\ln (E \Theta / \Lambda)}{\ln \left(E \Theta_{0} / \Lambda\right)}\right)^{\gamma}, \\
\langle C\rangle_{\infty} & =\alpha N_{c}+\beta C_{F},
\end{aligned}
$$

where $\quad \gamma=\frac{8}{3} C_{F}+\frac{2}{3} n_{f} ; \quad \alpha=\frac{8}{3} \frac{C_{F}}{\gamma}, \quad \beta=\frac{2}{3} \frac{n_{f}}{\gamma}, \quad \alpha+\beta=1$.

Equations (9.12) describe how a registered parton $B$ looses the memory about the color charge of a parent parton $A$, when the aperture $\Theta$ decreases. At $E \rightarrow \infty$ and $\Theta \rightarrow 0$ one has $\langle C\rangle_{q}=\langle C\rangle_{g}=\langle C\rangle_{\infty}=2.4$ for $n_{f}=3$ ( 2.12 for $n_{f}=6$ ). This limiting color current proves to be, naturally, somewhere in between 


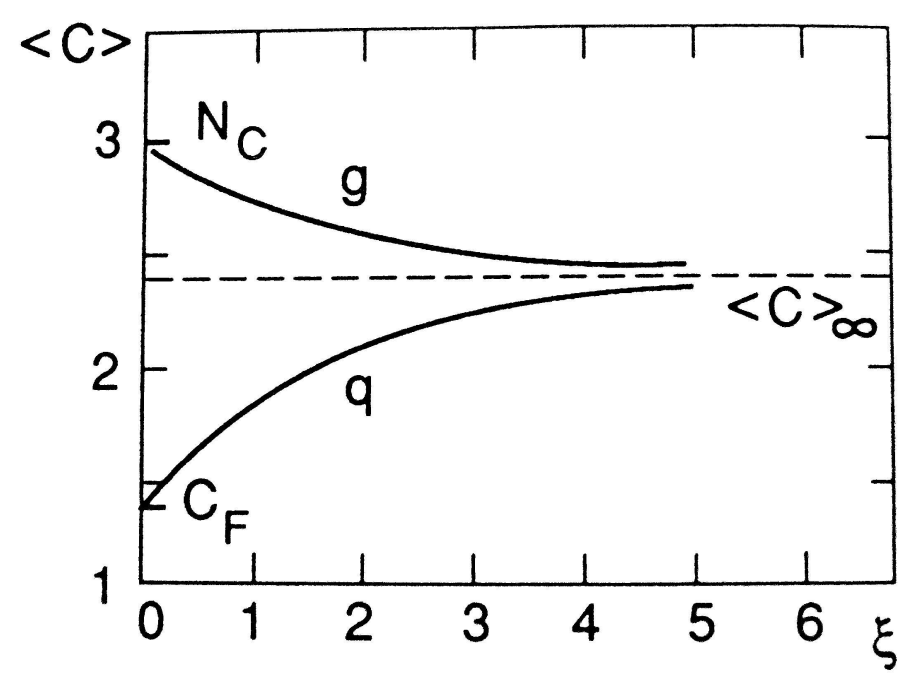

Figure 9.3: The mean parton color current in $q$ and $g$ jets with hardness $E \Theta_{0}$ as registered by the calorimeter with angular aperture $\Theta$; $\xi=\ln \ln E \Theta_{0} / \Lambda-\ln \ln E \Theta / \Lambda$.

the gluon and the quark charges. Therefore, for a gluon jet the "color-grasp" of an emitter decreases with $\Theta$ decreasing, and for a quark jet this quantity increases, as shown in Fig. 9.3.

It is of interest to note that, while the ratio of the total mean multiplicities in $g$ and $q$ jets asymptotically equals $C_{g} / C_{F}=9 / 4$, for the case of a narrow cone of observation this ratio tends to 1 .

The dependence of energy distribution on the aperture of the registered particle flow, as given by (9.6), was presented in Fig. 7.1. The narrower is the registration cone, the harder are particles within it, $x>x_{\min }=Q_{0} / E \Theta$. 
On the other hand, (9.6) describes the average energy flux deposited within a cone $\Theta$ around the registered hadron $h$ carrying an energy fraction $x$. As can be seen from the figure, a soft hadron with $x \ll 1$ is accompanied by the energy flux only starting from sufficiently large values of the calorimeter aperture,

$$
\Theta>\Theta_{\min }=Q_{0} / x E .
$$

\subsubsection{Collimation of Multiplicity Inside Jet}

By analogy, one can ask what is the angular size $\Theta$ of the cone, where the bulk of jet multiplicity is concentrated, and what is the energy behavior of this aperture. To answer this question quantitatively one should solve the equation

$$
N_{A}^{h}\left(\Theta_{\delta} ; E, \Theta_{0}\right)=\delta \cdot N_{A}^{h}\left(E \Theta_{0}\right)
$$

and find the value of the angle $\Theta_{\delta}$, where the share $\delta$ of the total multiplicity is concentrated. Then accounting for the DL relation $N_{A}\left(E \Theta_{0}\right)=C_{A} / N_{c}$. $N_{g}\left(E \Theta_{0}\right)$ one gets from (9.11), (9.14) the equation for $\delta$ :

$$
\langle C\rangle_{A} N_{g}^{h}\left(E \Theta_{\delta}\right)=\delta \cdot C_{A} N_{g}^{h}\left(E \Theta_{0}\right) .
$$

With the help of (9.12) it can be written down in the form

$$
\left[a_{A}+b_{A}\left(\frac{\ln \left(E \Theta_{\delta} / \Lambda\right)}{\ln \left(E \Theta_{0} / \Lambda\right)}\right)^{\frac{50}{81}}\right] N_{g}^{h}\left(E \Theta_{\delta}\right)=\delta \cdot N_{g}^{h}\left(E \Theta_{0}\right),
$$

where $a_{q} \approx 1.8, b_{q} \approx 0.8$ and $a_{g} \approx 0.8, b_{q} \approx 0.2$.

Fig. 9.2 shows how appropriate cones shrink with increasing energy for two particular values of $\delta$. It can be easily seen that the multiplicity flow in a $q$-jet is collimated around the direction of the energy flux much stronger than 
in a $g$-jet. With the increasing of jet energy the collimation of the multiplicity flows grows more slowly than that of the energy flux.

Let us roughly estimate the multiplicity collimation taking $\Theta_{0} \sim 1$ :

$$
N\left(E \Theta_{\delta}\right)=\delta \cdot N(E), \quad \exp \sqrt{\frac{16 N_{c}}{b} \ln \frac{E \Theta_{\delta}}{\Lambda}}=\delta \cdot \exp \sqrt{\frac{16 N_{c}}{b} \ln \frac{E}{\Lambda}}
$$

which results in

$$
\Theta_{\delta} \sim[N(E)]-\frac{b}{8 N_{c}} \ln \frac{1}{\delta}
$$

Thus, the solid angle having half the jet multiplicity decreases with the increase of the hardness $\left(E \Theta_{0} \sim E\right)$ approximately as $N^{-1 / 4}(E)$, i.e. parametrically much slower than in the case of the collimation of energy.

\subsection{Multiplicity Flow Pattern for $q \bar{q} g$ Ensemble}

Here we shall describe the QCD portrait of the simplest multijet system, corresponding to the $q \bar{q} g$ sample of $e^{+} e^{-}$annihilation. The formulae which we derive in this Section to describe the total multiplicity in $q \bar{q} g$ events and the spatial distribution of particle flow, will include the next-to-leading MLLAcorrections of the order of $\sqrt{\alpha_{s}}$.

In terms of the inclusive approach a proper characteristic of the spatial distribution of the multiplicity flow is $E^{3} M C$ :

$$
\frac{d N_{4}}{d \Omega_{\vec{n}}}=\sum_{a, b, c} \int d E_{a} d E_{b} d E_{c} d E_{\vec{n}} \frac{E_{a} E_{b} E_{c} d \sigma_{4}}{\sigma_{2} d E_{a} d E_{b} d E_{c} d E_{\vec{n}} d \Omega_{a} d \Omega_{b} d \Omega_{c} d \Omega_{\vec{n}}}
$$




$$
\sigma_{3}=\sum_{a, b, c} \int d E_{a} d E_{b} d E_{c} \frac{E_{a} E_{b} E_{c} d \sigma_{3}}{d E_{a} d E_{b} d E_{c} d \Omega_{a} d \Omega_{b} d \Omega_{c}},
$$

where the sum runs over all particles. This represents an angular correlation between the three registered hard particles (a,b,c), moving in the directions $\vec{n}_{a}, \vec{n}_{b}$ and $\vec{n}_{c}$ and the multiplicity flow around the direction $\vec{n}$ as shown in Fig. 9.4

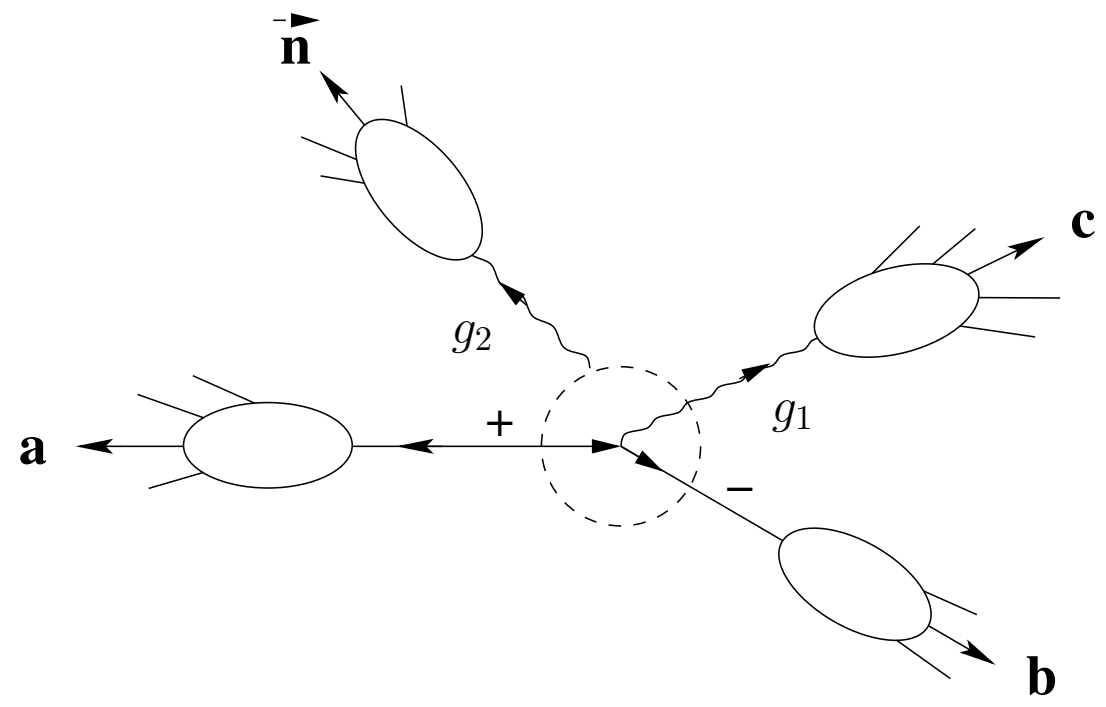

Figure 9.4: Angular inclusive correlation between three energetic $(a, b, c)$ and one soft particle $(\vec{n})$ in the $e^{+} e^{-}$annihilation process.

When all three vectors $\vec{n}_{a}, \vec{n}_{b}$ and $\vec{n}_{c}$ are in the same plane, the main contribution to $d N_{4}$ comes from the $q \bar{q} g$ configuration of the primary parton system. In the leading order in $\alpha_{s}$ the parton kinematics is unambiguously 
fixed as follows:

$$
\begin{gathered}
\vec{n}_{+} \approx \vec{n}_{a}, \quad \vec{n}_{-} \approx \vec{n}_{b}, \quad \vec{n}_{1} \approx \vec{n}_{c} \\
x_{+}=2 \frac{\sin \Theta_{1-}}{\sum \sin \Theta_{i j}}, \quad x_{-}=2 \frac{\sin \Theta_{1+}}{\sum \sin \Theta_{i j}}, \quad x_{1}=2 \frac{\sin \Theta_{+-}}{\sum \sin \Theta_{i j}}, \\
x_{+}+x_{-}+x_{1}=2,
\end{gathered}
$$

with $x_{i}=2 E_{i} / W$ the normalized parton energies and $\Theta_{i j}$ the angles between partons $i$ and $j\left(+,-\equiv q, \bar{q} ; \quad 1 \equiv g_{1}\right)$. We emphasize here that, owing to coherence, the radiation of a secondary gluon $g_{2}\left(k_{2} \ll E_{i}\right)$ at angles higher than the characteristic angular size of each parton jet proves to be insensitive to its internal structure: $g_{2}$ is emitted by the color current which is conserved when the jet splits. That is the reason why one may replace each jet by its parent parton with $p_{i}^{2} \approx 0$, as it was done when deriving (9.17).

\subsubsection{Radiation Pattern for $q \bar{q} \gamma$-events}

Let us turn firstly to the simpler case of two-jet events. Here the particle flow distribution corresponding to the correlation (9.2) can be written as

$$
\frac{8 \pi d N_{q \bar{q}}}{d \Omega_{\vec{n}}}=\frac{2}{a_{+}} N_{q}^{\prime}\left(Y_{+}, Y\right)+\frac{2}{a_{-}} N_{q}^{\prime}\left(Y_{-}, Y\right) .
$$

The factor $N_{A}^{\prime}\left(Y_{i}, Y\right) \equiv\left(d / d Y_{i}\right) N_{A}\left(Y_{i}, Y\right)$ takes into account that the final registered hadron is a part of cascade (cascading factor), where $N_{A}\left(Y_{i}, Y\right)$ stands for the multiplicity in a jet $A(A=q, g)$ of particles concentrated in the cone with an angular aperture $\Theta_{i}$ around the jet direction $\vec{n}_{i}$. In the 
above

$$
\begin{aligned}
a_{i} \equiv 1-\vec{n} \vec{n}_{i} \quad, \quad \vec{n}_{+} \approx \vec{n}_{a}, \quad \vec{n}_{-} \approx \vec{n}_{b} \approx-\vec{n}_{a}, \\
Y_{i} \equiv \ln \left(\frac{E \sqrt{a_{i} / 2}}{\Lambda}\right), \quad Y \equiv \ln \frac{E}{\Lambda},
\end{aligned}
$$

with $E=W / 2$ the jet energy. To understand the meaning of the quantity $N_{A}\left(Y_{i}, Y\right)$ it is helpful to represent it as

$$
\begin{gathered}
N_{A}\left(Y_{i}, Y\right)=\sum_{B=q, g} \int_{0}^{1} d z z \bar{D}_{A}^{B}(z, \Delta \xi) N_{B}\left(\overline{Y_{i}}\right), \\
\Delta \xi=\frac{1}{b} \ln \left(Y / \overline{Y_{i}}\right) \quad, \quad \overline{Y_{i}}=Y_{i}+\ln z=\ln \left(\frac{z E}{\Lambda} \sqrt{\frac{a_{i}}{2}}\right) .
\end{gathered}
$$

Here $N_{B}\left(\overline{Y_{i}}\right)$ is the multiplicity in a jet with the hardness scale $\overline{Y_{i}}$, initiated by a parton $B$ within the cone $\Theta_{i}$, and $\bar{D}_{A}^{B}$ denotes the structure function for parton fragmentation $A \rightarrow B$.

Equation (9.19) accounts for the fact that due to coherence the radiation at small angles $\Theta_{i} \ll 1$ is governed not by the overall color current of a jet $A$, but by that of a subjet $B$, developing inside a much narrower cone $\Theta_{i}$. This formula has a correct limit at $\Theta_{i} \rightarrow \pi$ :

$$
Y_{i} \rightarrow Y, \quad \Delta \xi \rightarrow 0, \quad \bar{D}_{A}^{B} \rightarrow \delta(1-z) \delta_{A}^{B}, \quad N_{A}\left(Y_{i}, Y\right) \rightarrow N_{A}(Y) .
$$

Equation (9.18) looks, formally, as the sum of two contributions, accounting for the independent evolution of the $q$ and $\bar{q}$ jets. However, one can see, that this reflects also the collective character of the soft radiation at large angles. Indeed, for large angles one obtains, neglecting relative corrections of order of $O\left(\alpha_{s}\right)$ :

$$
N_{q}^{\prime}\left(Y_{+}, Y\right) \approx N_{q}^{\prime}\left(Y_{-}, Y\right) \approx N_{q}^{\prime}(Y) \propto \sqrt{\alpha_{s}} N_{q}(Y) .
$$


Thus, (9.18) can be transformed to

$$
8 \pi \frac{d N_{q \bar{q}}}{d \Omega_{\vec{n}}}=2(\widehat{+-}) N_{q}^{\prime}(\ln E / \Lambda)
$$

where the notation of (4.13) is used $\left(a_{+}+a_{-}=a_{+-}=2\right)$.

Equation (9.20) represents the radiation pattern for the interjet gluon emission by the antenna $(\widehat{+-})$ described in Section 4.4. The cascading factor reads

$$
\frac{N_{c}}{C_{F}} \cdot N_{q}^{\prime}\left(\ln \frac{E}{\Lambda}\right) \approx N_{g}^{\prime}\left(\ln \frac{E}{\Lambda}\right)=\int^{E} \frac{d E_{g}}{E_{g}} 4 N_{c} \frac{\alpha_{s}\left(E_{g}\right)}{2 \pi} N_{g}\left(\ln \frac{E_{g}}{\Lambda}\right) .
$$

For the so called radiative two-jet events $\left(e^{+} e^{-} \rightarrow q \bar{q} \gamma\right)$ the emission pattern is given by the $q \bar{q}$ sample Lorentz boosted from the quark $\mathrm{cms}$ to the lab system (i.e. the $c m s$ of $q \bar{q} \gamma$ ), and the corresponding particle multiplicity should surely be equal to that in $e^{+} e^{-} \rightarrow q \bar{q}$ at $W_{q \bar{q}}^{2}=\left(p_{q}+p_{\bar{q}}\right)^{2}$.

By analogy with (9.18) the formula for the angular distribution of particle flow in $q \bar{q} \gamma$ events can be written as

$$
\frac{8 \pi d N_{q \bar{q} \gamma}}{d \Omega_{\vec{n}}}=\frac{2}{a_{+}} N_{q}^{\prime}\left(Y_{q+}, Y_{q}\right)+\frac{2}{a_{-}} N_{q}^{\prime}\left(Y_{\bar{q}-}, Y_{\bar{q}}\right)+2 I_{+-} N_{q}^{\prime}(Y)
$$

where

$$
Y_{q(\bar{q})}=\ln \frac{E_{q(\bar{q})}}{\Lambda}, \quad Y_{q+}=\ln \left(\frac{E_{q} \sqrt{a_{+} / 2}}{\Lambda}\right), \quad Y_{\bar{q}-}=\ln \left(\frac{E_{\bar{q}} \sqrt{a_{-} / 2}}{\Lambda}\right)
$$

and

$$
\left.I_{+-}=\widehat{(+-}\right)-\frac{1}{a_{-}}-\frac{1}{a_{+}}=\frac{a_{+-}-a_{+}-a_{-}}{a_{+} a_{-}} .
$$

For the emission at large angles $\left(a_{+} \sim a_{-} \sim 1\right)$ when all the factors $N^{\prime}$ are approximately the same, (9.21) coincides with (9.20). 


\subsubsection{Radiation Pattern for $q \bar{q} g$-events}

We are ready now to deal with the three-jet event sample when a hard photon is replaced by a gluon $g_{1}$. For a given $q \bar{q} g_{1}$ configuration the particle flow can be presented, analogously to (9.21)-(9.23), as

$$
\begin{array}{r}
\frac{8 \pi d N_{q \bar{q} g}}{d \Omega_{\vec{n}}}=\frac{2}{a_{+}} N_{q}^{\prime}\left(Y_{q+}, Y_{q}\right)+\frac{2}{a_{-}} N_{q}^{\prime}\left(Y_{\bar{q}-}, Y_{\bar{q}}\right)+\frac{2}{a_{1}} N_{g}^{\prime}\left(Y_{g 1}, Y_{g}\right) \\
+2\left[I_{1+}+I_{1-}-\left(1-\frac{2 C_{F}}{N_{c}}\right) I_{+-}\right] N_{g}^{\prime}(Y),
\end{array}
$$

where, in addition to the definitions in (9.22),

$$
Y_{g}=\ln \frac{E_{g}}{\Lambda}, \quad Y_{g 1}=\ln \left(\frac{E_{g} \sqrt{a_{1} / 2}}{\Lambda}\right) .
$$

This formula accounts for both types of coherence: the angular ordering inside each of the jets and the collective nature of the interjet flows. The first three terms in (9.24) are collinear singular as $\Theta_{i} \rightarrow 0$ and contain the factors $N^{\prime}$, describing the evolution of each jet initiated by the hard emitters $q, \bar{q}$ and $g_{1}$. The last term accounts for the interference between these jets. It has no collinear singularities and contains the common factor $N_{g}^{\prime}(Y, Y)$ independent of the direction $\vec{n}$.

Fig. 9.5illustrates the predicted distribution of particle flow projected onto the event plane for the Mercedes-like $q \bar{q} g$ events. It seems to be of importance to observe experimentally the energy dependence of the multiplicity flow, as demonstrated in Fig. 9.5.

As follows from (9.21), (9.24) when replacing a hard photon by a gluon $g_{1}$, with otherwise identical kinematics, an additional particle flow arises

$$
\left(\frac{8 \pi d N}{d \Omega_{\vec{n}}}\right)_{g}=\frac{8 \pi d N_{q \bar{q} g}}{d \Omega_{\vec{n}}}-\frac{8 \pi d N_{q \bar{q} \gamma}}{d \Omega_{\vec{n}}}=\frac{2}{a_{1}} N_{g}^{\prime}\left(Y_{g 1}, Y_{g}\right)+\left[I_{1+}+I_{1-}-I_{+-}\right] N_{g}^{\prime}(Y) \text {. }
$$




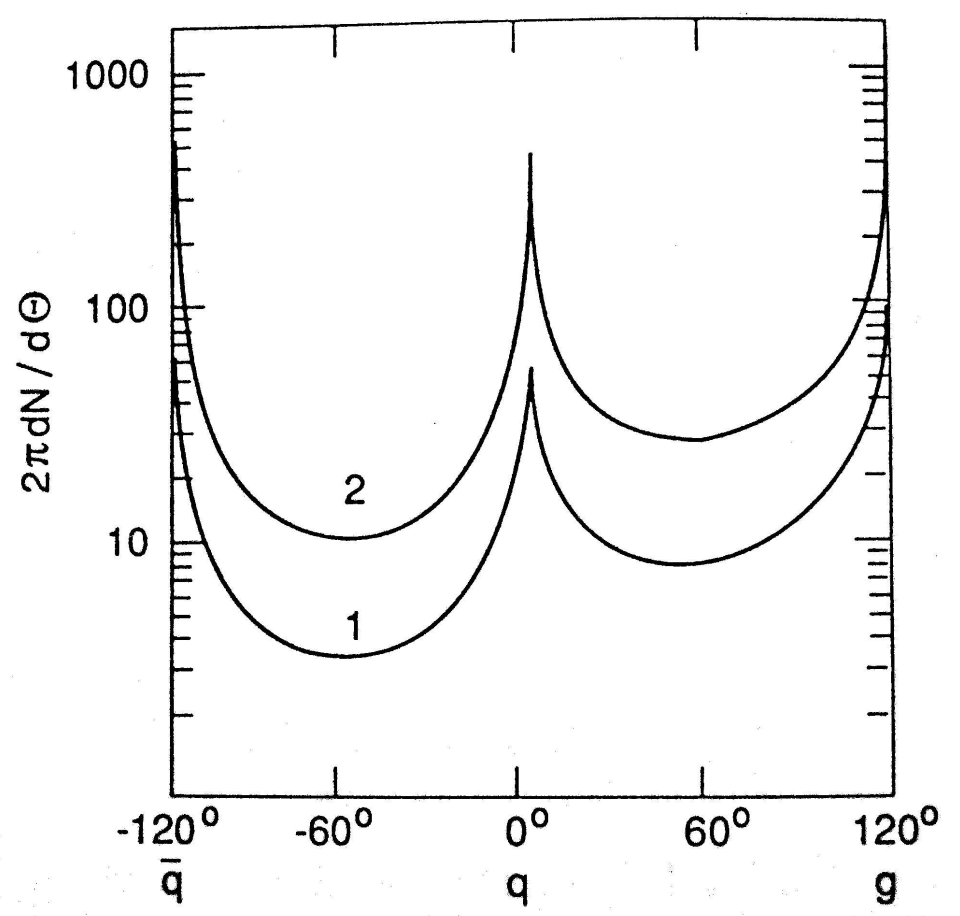

Figure 9.5: Particle angular flows in the three-fold symmetric $q \bar{q} g$ events on the event plane with respect to the $q$-jet axis at different values of the scale parameter $E / \Lambda=60(1), 1000(2)$. 
Note that for the case of large radiation angles both cascading factors $N^{\prime}$ become approximately equal and one has

$$
\left(\frac{8 \pi d N}{d \Omega_{\vec{n}}}\right)_{g}=[\widehat{(1+)}+\widehat{(1-)}-\widehat{(+-)}] \cdot N_{g}^{\prime}(Y) .
$$

An interesting point is that this expression which seems to look like a "true" gluon contribution is not positively definite. One can observe the net destructive interference in the region between the $q$ and $\bar{q}$ jets. The soft radiation in this direction proves to be less than that in the absence of the gluon jet $g_{1}$.

The drag phenomena are clearly seen in the experiments (see Refs. [4:710]21]23]) thus supporting both QCD prediction and the LPHD concept. The physics of drag effect will be discussed in more details in the next Section.

\subsubsection{Average Multiplicity of 3-jet events}

Let us consider now the connection of the average particle multiplicities in the two-jet and three-jet samples of $e^{+} e^{-}$annihilation. Recall that the particle multiplicity in an individual quark jet was formally defined through that in $e^{+} e^{-} \rightarrow q \bar{q} \rightarrow$ hadrons by (see Section 7.9)

$$
N_{e^{+} e^{-}}^{c h}(W)=2 \cdot N_{q}^{c h}(E) \cdot\left[1+O\left(\frac{\alpha_{s}(W)}{\pi}\right)\right], \quad W=2 E .
$$

When three or more partons are involved in a hard interaction, say, $e^{+} e^{-} \rightarrow$ $q \bar{q} g_{1}$, the multiplicity can not be represented simply as a sum of three independent parton contributions. The point is that multiplicity becomes dependent on the geometry of the whole jet ensemble. 
So, the problem arises of describing the multiplicity in three-jet events, $N_{q \bar{q} g}$, in terms of the characteristics of the individual $q$ and $g$ jets discussed above. The quantity $N_{q \bar{q} g}$ should depend on the $q \bar{q} g$ geometry in a Lorentzinvariant way and should have a correct limit when the event is transformed to the two-jet configuration by decreasing either the energy of the gluon $g_{1}$ or its emission angle.

Note that to derive the formula for $N_{q \bar{q} g}$ accounting for the interjet contribution, one needs to control systematically the relatively small $\sim \sqrt{\alpha_{s}}$ terms. Formally, the MLLA analysis does not provide such an accuracy, since the change in $\Lambda$, or in the scale of $W$, by a finite factor $O(1)$ would lead to a correction in multiplicity

$$
\frac{\Delta N}{N} \sim O\left(\sqrt{\alpha_{s}(W)}\right)
$$

Such corrections could be formally absorbed into the definition of the scale parameter $\Lambda$, the value of which should be fixed phenomenologically from comparison of the MLLA partonic spectra with measured ones. In what follows we shall use the MLLA formula for jet multiplicity given by (17.32), (7.35) with the just that very value of $\Lambda$. This permits one to predict the energy dependence of the hadron multiplicities including terms $\sim \sqrt{\alpha_{s}} N$.

Integration of (9.18) can be easily checked to reproduce the total multiplicity. The angular integral of (9.21) can be written as

$$
N_{q \bar{q} \gamma}=\int \frac{d N_{q \bar{q} \gamma}}{d \Omega_{\vec{n}}}=N_{q}\left(Y_{q}\right)+N_{\bar{q}}\left(Y_{\bar{q}}\right)+2 \ln \sqrt{\frac{a_{+-}}{2}} \cdot N_{q}^{\prime}(Y) .
$$

Now we can transform this formula to the Lorentz-invariant expression. To do that let us rewrite

$$
Y_{q(\bar{q})}=Y+\ln x_{+(-)} \quad, \quad x_{+(-)} \equiv E_{q(\bar{q})} / E
$$


and use the expansion

$$
N_{q}\left(Y_{q}\right)=N_{q}(Y)+\ln x_{+} \cdot N_{q}^{\prime}(Y)+O\left(\frac{\alpha_{s}}{\pi} N_{q}\right) .
$$

Then

$$
\begin{gathered}
N_{q \bar{q} \gamma}=2 N_{q}(Y)+\ln \frac{x_{+} x_{-} a_{+-}}{2} \cdot N_{q}^{\prime}(Y)+O\left(\alpha_{s} N\right)=2 N_{q}\left(Y_{+-}^{*}\right) \cdot\left[1+O\left(\alpha_{s}\right)\right] \\
\quad \text { with } \quad Y_{+-}^{*}=Y+\ln \sqrt{\frac{x_{+} x_{-} a_{+-}}{2}}=\ln \sqrt{\frac{\left(p_{+} p_{-}\right)}{2 \Lambda^{2}}}=\ln \frac{E^{*}}{\Lambda}
\end{gathered}
$$

Here $E^{*}$ is the quark energy in the $\mathrm{cms}$ of $q \bar{q}$, i.e. the Lorentz-invariant generalization of a true parameter of hardness of the process.

The multiplicity $N_{q \bar{q} g}$ is, by analogy,

$$
\begin{array}{r}
N_{q \bar{q} g}=\int \frac{d N_{q \bar{q} g}}{d \Omega_{\vec{n}}}=N_{q}\left(Y_{q}\right)+N_{\bar{q}}\left(Y_{\bar{q}}\right)+N_{g}\left(Y_{g}\right)+ \\
{\left[\ln \sqrt{\frac{a_{1+} a_{1-}}{2 a_{+-}}}+\frac{2 C_{F}}{N_{c}} \ln \sqrt{\frac{a_{+-}}{2}}\right] \cdot N_{g}^{\prime}(Y),}
\end{array}
$$

where $Y_{g}=Y+\ln x_{1}$. Proceeding as before one comes finally to the Lorentzinvariant result

$$
N_{q \bar{q} g}=\left[2 N_{q}\left(Y_{+-}^{*}\right)+N_{g}\left(Y_{g}^{*}\right)\right] \cdot\left(1+O\left(\frac{\alpha_{s}}{\pi}\right)\right),
$$

with

$$
Y_{g}^{*}=\ln \sqrt{\frac{\left(p_{+} p_{1}\right)\left(p_{-} p_{1}\right)}{2\left(p_{+} p_{-}\right) \Lambda^{2}}}=\ln \frac{p_{1 \perp}}{2 \Lambda},
$$

with $p_{1 \perp}$ the transverse momentum of $g_{1}$ in the $q \bar{q} \mathrm{cms}$. 
Comparing (9.28) with (9.29) we see, that replacement of a photon $\gamma$ by a gluon $g_{1}$ leads to the additional multiplicity

$$
N_{g}\left(Y_{g}^{*}\right)=N_{q \bar{q} g}(W)-N_{q \bar{q} \gamma}(W),
$$

which depends not on the gluon energy but on its transverse momentum, i.e. on the hardness of the primary process. Equation (9.29) reflects the coherent nature of soft emission and has a correct limit when the event is transformed to the two-jet configuration.

The same result can be written in another form, namely,

$$
N_{q \bar{q} g}=\left[2 N_{q}\left(Y_{+-}^{*}\right)+N_{g}\left(Y_{1+}^{*}\right)+N_{g}\left(Y_{1-}^{*}\right)-N_{g}\left(Y_{+-}^{*}\right)\right] \cdot\left[1+O\left(\alpha_{s}\right)\right],
$$

where $Y_{i j}^{*}=\ln \left(\sqrt{\left(p_{i} p_{j}\right) / 2 \Lambda^{2}}\right)=\ln \left(E_{i j}^{*} / \Lambda\right)$. This representation deals with multiplicities of two-jet events at appropriate invariant pair energies $E_{i j}^{*}$. Expression (9.32) has also a proper limit, $2 N_{g}$, when the $q \bar{q} g$ configuration is forced to a quasi-two-jet one, $g+(q \bar{q})$, with a the small angle between the quarks.

Let us illustrate the results for $N_{q \bar{q} g}$ with the case of the Mercedes-like $q \bar{q} g$ events $\left(\Theta_{q \bar{q}}=\Theta_{q g}=\Theta_{\bar{q} g} \equiv \Theta_{0}=120^{\circ}\right)$. According to (9.29) we get

$$
N_{q \bar{q} g}^{s y m}=\sum_{A=q, \bar{q}, g} N_{A}\left(Y^{*}\right), \quad Y^{*}=\ln \left(\frac{W}{3 \Lambda} \sin \frac{\Theta_{0}}{2}\right) .
$$

Here $N_{A}\left(Y^{*}\right)$ describes the particle multiplicity in a jet $A$ with the energy $E_{A}=W / 3$ and the opening angle $\Theta_{0}$. Now we define the angular regions in the event plane, as shown in Fig. 9.6, and with use of (9.33) calculate the number of partons found in these regions. The result is presented in Table 9.1.

Let us draw attention that the ratio $R$ of particle numbers in the regions, which could be associated with the gluon and quark jets, is lower than its 


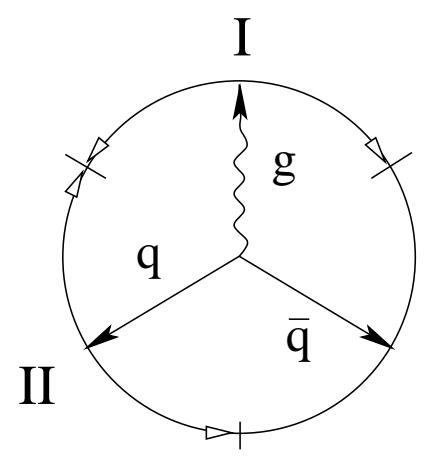

Figure 9.6: Angular regions on the $q \bar{q} g$ plane for the Mercedes-like events, which could be associated with a gluon jet $(I)$ and a quark jet $(I I)$.

asymptotic value $24 R_{\infty}=N_{c} / C_{F}=2.25$ and rises rather slowly with energy increasing. Thus, at present energies the collective QCD effects in the spatial distributions of multiplicity flows lead to a decrease of the observed ratios of hadron yields in $g$ and $q$ jets.

Finally let us mention that in the first experiments the observed effects were actually rather small since it was difficult to separate the quark and

\begin{tabular}{|r||c|r|r|c|}
\hline$W / \Lambda$ & $N_{q \bar{q} g}^{t o t}$ & $N_{I}$ & $N_{I I}$ & $R=N_{I} / N_{I I}$ \\
\hline \hline 180 & 14 & 6.6 & 3.6 & 1.8 \\
\hline 300 & 19 & 9.2 & 4.9 & 1.9 \\
\hline 3000 & 56 & 28.0 & 14.0 & 2.0 \\
\hline
\end{tabular}

Table 9.1: Multiplicity distribution in the threefold symmetric $q \bar{q} g$ event over regions shown in Fig. 9.6. 
gluon jets. So the true effect was reduced by the influence of events where gluon was misidentified. This hampered the detailed experimental study of QCD drag phenomena.

With the high statistics at the $Z^{0}$ resonance one can use some prospective methods for jet identification. For example, the large rates of charm and bottom production permit one to tag the quark or/and antiquark of the $Q \bar{Q} g$ $(Q=c, b)$ event by the characteristic features of $Q$ decays (prompt leptons, specific exclusive final states, vertex detection, etc.), see Refs. 925. Quark tagging through the use of the semi-leptonic decays of $c$ and $b$ quarks for a study of drag phenomena was successfully performed recently at LEP 11 . Another very promising way which seemingly guarantees at least $85 \%$ of $g$ tagging was suggested which uses the computer "neural networks" trained to recognize the jet type 26.

An important point is that the drag phenomena appear to be the same for light and heavy quarks. This stems from the fact that the radiation off a quark at large angles, determining intERjet particle flows, proves to be insensitive to the value of the quark mass. Therefore one can use the standard PT formulae for the drag effect (see below) to study interjet physics with help of heavy quarks.

\subsection{QCD Drag Effect in Interjet Particle Flows}

Now we turn to particle flows at large angles to the jets in $e^{+} e^{-} \rightarrow q \bar{q} g$. Let all the angles $\Theta_{i j}$ between jets and the jet energies $E_{i}$ be large: $\Theta_{+-} \sim \Theta_{+1} \sim$ $\Theta_{-1} \sim 1, E_{1} \sim E_{+} \sim E_{-} \sim E \sim W / 3$. As was discussed above, the angular distribution of soft interjet hadrons carries information about the coherent 
gluon radiation off the color antenna formed by three emitters $(q, \bar{q}$ and $g)$.

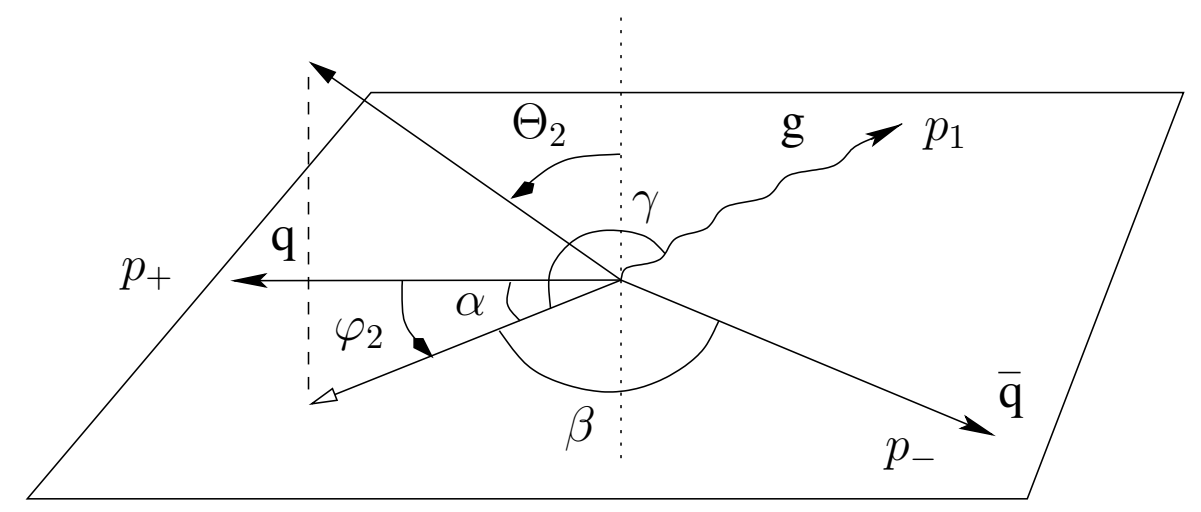

Figure 9.7: Kinematics of interjet radiation in three-jet events.

The wide-angle distribution of a secondary soft gluon $g_{2}$ displayed in Fig. 9.7 can be written according to (9.24) $-(9.25)$ as

$$
\frac{8 \pi d N_{q \bar{q} g}}{d \Omega_{\vec{n}_{2}}}=\frac{1}{N_{c}} W_{ \pm 1}\left(\vec{n}_{2}\right) N_{g}^{\prime}\left(Y_{m}\right)=\left[\widehat{(1+)}+\widehat{(1-)}-\frac{1}{N_{c}^{2}}(\widehat{+-})\right] N_{g}^{\prime}\left(Y_{m}\right) .
$$

In the above $Y_{m}=\ln E \Theta_{m} / \Lambda, \Theta_{m}=\min \left\{\Theta_{+}, \Theta_{-}, \Theta_{1}\right\}$ with $\cos \Theta_{i}=\vec{n}_{2} \vec{n}_{i}$. The radiation pattern corresponding to the case when a photon $\gamma$ is emitted instead of a gluon reads ( $c f .(9.20)$ )

$$
\frac{8 \pi d N_{q \bar{q} \gamma}}{d \Omega_{\vec{n}_{2}}}=\frac{1}{N_{c}} W_{+-}\left(\vec{n}_{2}\right) N_{g}^{\prime}\left(Y_{m}\right)=\frac{2 C_{F}}{N_{c}}(\widehat{+-}) \cdot N_{g}^{\prime}\left(Y_{m}\right) .
$$

The dashed line in Fig. 9.8 displays the corresponding "directivity diagram", 


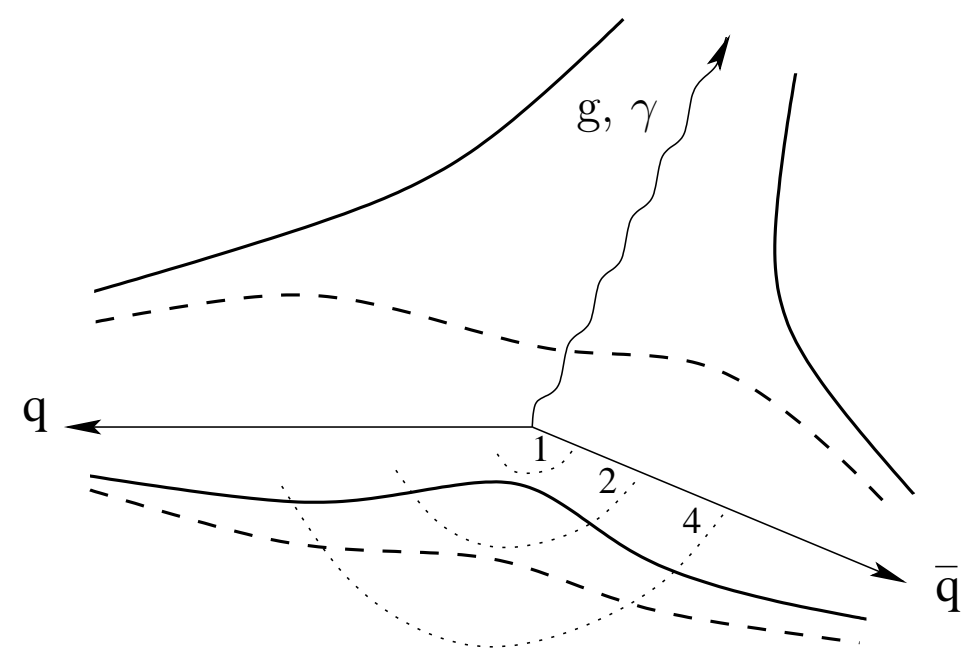

Figure 9.8: Directivity diagram of soft gluon radiation, projected onto the $q \bar{q} \gamma$ (dashed line) and $q \bar{q} g$ (solid) event planes. Particle flows (9.36) and (9.37) are drawn in polar coordinates: $\Theta=\phi_{2}, \quad r=\ln 2 W\left(\phi_{2}\right)$. Dotted circles show the constant levels of particle flow: $W\left(\phi_{2}\right)=1,2,4$.

projected onto the $q \bar{q} \gamma$ plane:

$$
\begin{array}{r}
W_{+-}\left(\phi_{2}\right)=2 C_{F} \int \frac{d \cos \Theta_{2}}{2}(\widehat{+-})=2 C_{F} a_{+-} V(\alpha, \beta), \\
V(\alpha, \beta)=\frac{2}{\cos \alpha-\cos \beta}\left(\frac{\pi-\alpha}{\sin \alpha}-\frac{\pi-\beta}{\sin \beta}\right) ; \alpha=\phi_{2}, \beta=\Theta_{+-}-\phi_{2} .
\end{array}
$$

Expression $W_{+-}\left(\vec{n}_{2}\right)$ is simply related to the particle distribution in the twojet events $e^{+} e^{-} \rightarrow q\left(p_{+}\right)+\bar{q}\left(p_{-}\right)$, Lorentz boosted from the quark $\mathrm{cms}$ to the lab system. 
Replacing $\gamma$ by $g_{1}$ changes the directivity diagram essentially because the antenna element $g_{1}$ now participates in the emission as well. However, contrary to naive intuition, this change does not lead only to appearance of an additional particle flow in the $g_{1}$ direction. Integrating (2.34) over $\Theta_{2}$ one obtains $\left(\gamma=\Theta_{1+}+\phi_{2}\right)$ :

$$
W_{ \pm 1}\left(\phi_{2}\right)=N_{c}\left[a_{+1} V(\alpha, \gamma)+a_{-1} V(\beta, \gamma)-\frac{1}{N_{c}^{2}} a_{+-} V(\alpha, \beta)\right] .
$$

Fig. 9.8 illustrates that the particle flow in the direction opposite to $\vec{n}_{1}$ appears to be considerably lower than in the photon case. So, the destructive interference cancels radiation in the region between the quark jets giving a surplus of radiation in the $q-g$ and $\bar{q}-g$ valleys.

To demonstrate this drag effect let us take $\vec{n}_{+} \vec{n}_{-}=\vec{n}_{+} \vec{n}_{1}=\vec{n}_{-} \vec{n}_{1}$ and $\vec{n}_{2}$ pointing at direction opposite to $\vec{n}_{1}$, that is, midway between quarks. Then, neglecting the weak dependence $N_{g}^{\prime}$ on $\Theta$, one arrives at the ratio

$$
\frac{d N_{q \bar{q} g} / d \vec{n}_{2}}{d N_{q \bar{q} \gamma} / d \vec{n}_{2}}=\frac{N_{c}^{2}-2}{2\left(N_{c}^{2}-1\right)} \approx 0.44 .
$$

We emphasize that (9.24) and (9.34) provide not only the planar picture, but the global three-dimensional pattern of particle flows.

It is worth noting that the destructive interference proves to be strong enough to dump the particle flow in the direction opposite to the gluon to even smaller values than that in the most kinematically "unfavorable" direction, which is transverse to the event plane. The asymptotic ratio of these flows in the case of threefold symmetric $q \bar{q} g$ events is

$$
\frac{N_{\langle\perp\rangle}}{N_{\langle q \bar{q}\rangle}}=\frac{N_{c}+2 C_{F}}{2\left(4 C_{F}-N_{c}\right)} \approx 1.2
$$


(see also Section 4.4).

Thus, the analysis of the bremsstrahlung pattern demonstrates particle "drag" by the gluon jet $g_{1}$. This phenomenon is easy to understand quantitatively. If one drops the color suppressed contribution, the two remaining terms in (9.34) may be interpreted as the sum of two independent $\widehat{(1+)}$ and $\widehat{(1-)}$ antenna patterns, boosted from their respective rest frames into the overall $q \bar{q} g \mathrm{cms}$.
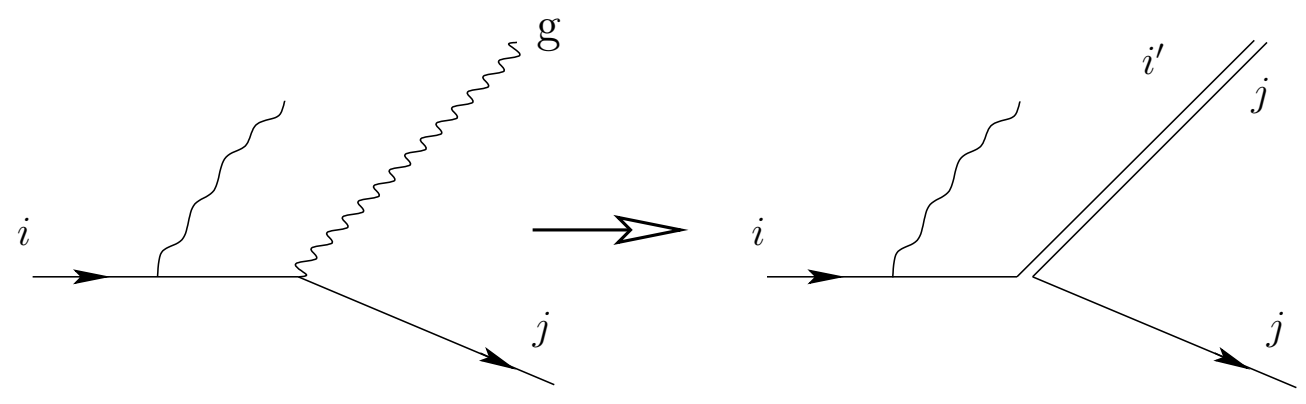

Figure 9.9: Emission of a soft gluon in the large $-N_{c}$ approximation.

The point is, that with neglecting the $1 / N_{c}^{2}$ terms, the hard gluon can be treated as a quark-antiquark pair as shown in Fig. 9.9, In this approximation each external quark line is uniquely connected to an external antiquark line of the same color, forming what we have called colorless $q \bar{q}$ antennae. In the general case, when calculating the resultant soft radiation pattern, one can only deal with a set of such color connected $q \bar{q}$ pairs because the interference between gluons emitted from non-color-connected lines proves to be suppressed by powers of $1 / N_{c}^{2}$.

Along this line of reasoning the depletion of the $q-\bar{q}$ valley is a direct 
consequence of Lorentz boosts. Such a scenario reproduces literally the explanation of drag/string effect given by the canonical Lund string model (see Refs. 827). In this model quark and antiquark are supposed to be tied together by a nonperturbative string qualitatively similar to a superconducting flux tube.

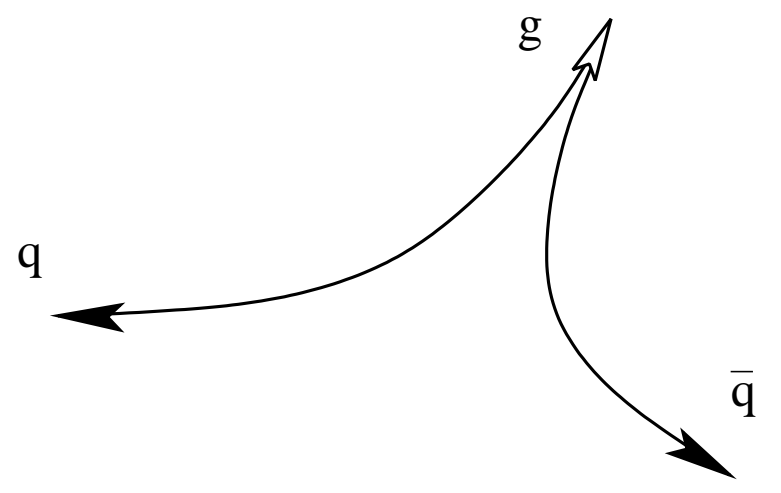

Figure 9.10: Canonical string model picture for the $e^{+} e^{-} \rightarrow q \bar{q} g$ event.

The gluon is pictured here as a kink on this string, see Fig. 9.10 Two string pieces in Fig. 9.10 are boosted from their respective rest frames and because of this hadrons resulting from fragmentation of strings tend to flow towards the gluon side. Notice that since there is no string piece spanned directly between the quark and antiquark, no particles are produced in between them, except by some minor "leakage" from the other two regions.

So we see that in what concerns $q \bar{q} g$ physics, as well as in many other cases, the Lund string scenario provides an excellent picture for mimicking the collective QCD phenomena that reflect dynamics of color flows. However, at some point significant differences should certainly come out. First of all, 
one might expect serious discrepancy between QCD and the string model when studying some specific phenomena where color non-leading contributions dominate, and such happenings do take place, as we shall see later.

Now turning back to the $q \bar{q} g$ events let us notice that the canonical string scheme predicts a larger drag for heavier hadrons and for particles carrying a larger momentum component perpendicular to the event plane, $p_{h}^{\text {out }}$. The reasons are the following. The Lorentz transformation of momentum $\overrightarrow{p_{i}}$ along the boost direction is given by

$$
\overrightarrow{p_{i}} \vec{n}_{B} \rightarrow \gamma_{B}\left(\vec{p}_{i} \vec{n}_{B}+\beta_{B} E_{i}\right),
$$

where $\vec{n}_{B}$ is a unit vector in the boost direction, $\gamma_{B}$ and $\beta_{B}$ denote the $\gamma-$ factor and the boost speed, and $E_{i}$ stands for the particle energy. Since $E_{i}=\sqrt{p_{i}^{2}+m_{i}^{2}}$ and $p_{i}^{2}=\left(p_{i}^{\text {in }}\right)^{2}+\left(p_{i}^{\text {out }}\right)^{2}$, the effect becomes larger as $m_{h}$ or $p_{h}^{\text {out }}$ gets larger.

By contrast, in the perturbative approach there are no reasons to expect any substantial enhancement for subsamples with large $p_{h}^{\text {out }}$ or $m_{h}$ since, provided the energy is high enough for QCD cascades to dominate, the yields of hadrons with different masses should be similar. In what concerns the $p_{h}^{\text {out }}$ dependence, even an opposite statement could be made: with increase of $p_{h}^{\text {out }}$ one leaves the event plane and the ratio of QCD motivated particle flows in $q-g$ and $q-\bar{q}$ valleys can be easily shown to approach unity instead of becoming larger 9 .

So, while the subasymptotic fragmentation effects may be important at low energies, with increasing energy the QCD picture has to win out. Monte Carlo studies of the energy evolution of the hadron flow ratios for subsamples with different $p_{h}^{\text {out }}$ and $m_{h}$ show that the perturbative regime becomes dominant already at LEP energies (see Refs. [28,29] for details). Experiments 4 [7]10 confirmed the drag effect in three-jet events. The depletion of particles was observed in the $q-\bar{q}$ valley relative to $q-g$ and $\bar{q}-g$ valleys. 


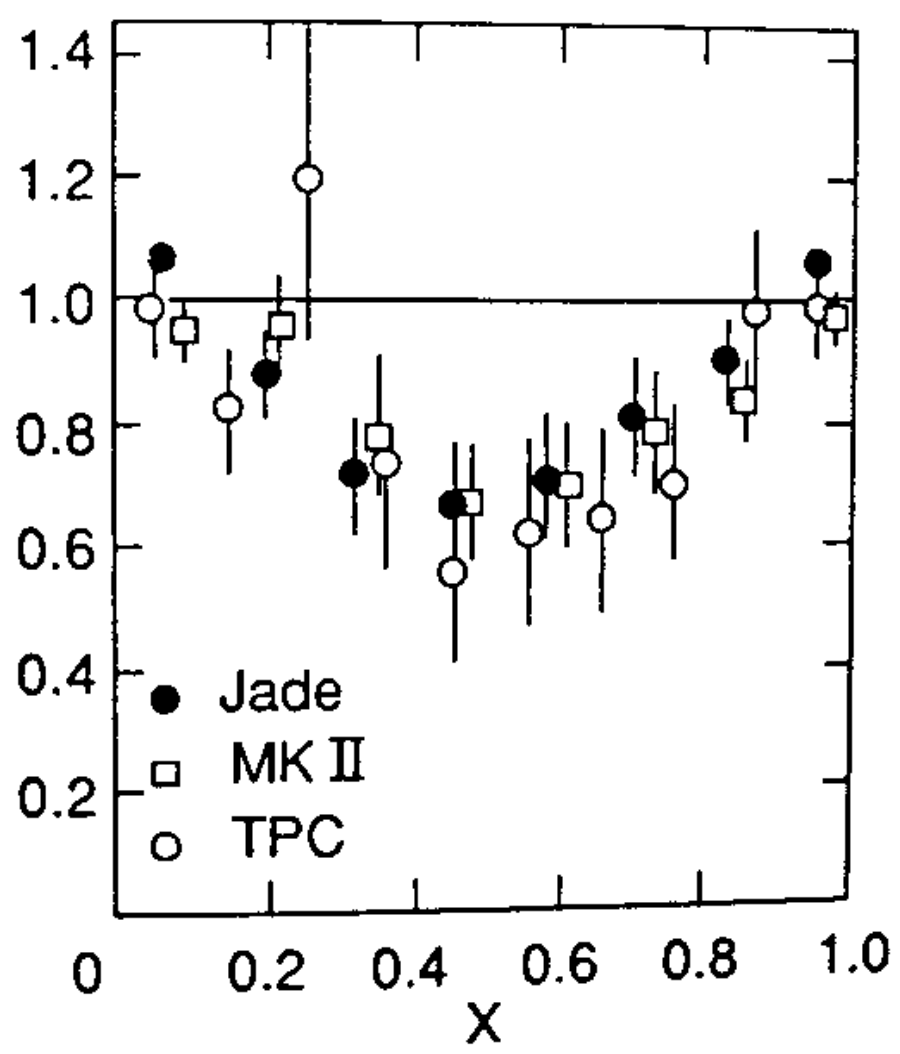

Figure 9.11: Ratio of particle densities in $q \bar{q} g$ and $q \bar{q} \gamma$ events as a function of the scaled angle $x=\Theta_{+} / \Theta_{+-}$. Shown are data from JADE, MARK II and TPC 21. 
The strong support of this effect came from the comparison of $q \bar{q} g$ and $q \bar{q} \gamma$ events 2223 , that provided a model independent test of color coherence. Fig. 9.11 shows the measured ratio of particle density in the $q-\bar{q}$ region for $q \bar{q} g$ and $q \bar{q} \gamma$ events. If no coherence effects are present this ratio should be 1 , since kinematical configuration of both event types are taken the same.

These observations have beautifully demonstrated the connection between color and hadronic flows. However one should take the standard analysis of 3jet events with a pinch of salt. The point is that it suffers from some inherent weaknesses that one would prefer to avoid.

First of all, the necessity of selecting a 3-jet event sample reduces the statistics and may introduce biases into the observed hadron flows. The need to define jet directions introduces a dependence on the jet-finding algorithm. Discrimination between quark and gluon jets (e.g., on the basis of their relative energies) reduces the effect and prevents the use of symmetrical jet configurations. Of course, the interjet collective phenomena are better analyzed if one identifies the quark jets. As it was mentioned in the previous Section, this can be done in the case of the heavy flavor production events by tagging heavy quark decays.

Another approach has been proposed in Ref. 15] for revealing the connection between the observed hadronic distributions and the color structure of an underlying hard process. The method is related to the drag effect analysis but has the advantage of not requiring any special event selection or jet finding. It involves measuring a ratio of energy-multiplicity correlations which is especially sensitive to color flow in jet formation. This quantity is infrared stable and can be calculated completely perturbatively.

To illustrate this approach, consider the emission of two soft gluons by a 
hard $q \bar{q}$ system

$$
W^{q \bar{q}}\left(\vec{n}_{3}, \vec{n}_{4}\right) \propto 2 C_{F} N_{c}\left(\frac{a_{12}}{a_{31} a_{43} a_{42}}+\frac{a_{12}}{a_{32} a_{43} a_{41}}-\frac{1}{N_{c}^{2}} \frac{a_{12}^{2}}{a_{31} a_{32} a_{41} a_{42}}\right) .
$$

Dividing by the product of the single-gluon distributions gives the gluon-gluon correlation function

$$
C^{q \bar{q}}\left(\eta_{34}, \phi\right)=\frac{W^{q \bar{q}}\left(\vec{n}_{3}, \vec{n}_{4}\right)}{W^{q \bar{q}}\left(\vec{n}_{3}\right) W^{q \bar{q}}\left(\vec{n}_{4}\right)}=1+\frac{N_{c}}{2 C_{F}}\left(\frac{\cos \phi}{\cosh \eta_{34}-\cos \phi}\right),
$$

$c f$. (4.26)) (for definitions of the angular variables $\eta_{34}, \phi$ see Section 4.6). Note that this asymptotic prediction can be numerically affected by finite energy subleading correction $\sim \sqrt{\alpha_{s}(W)}$.

Equation (9.41) provides an infrared-finite measure of the correlation between color flows in the directions $\eta_{3}, \phi_{3}$ and $\eta_{4}, \phi_{4}$. According to the LPHD hypothesis, it can be applied directly to hadronic flows. Considering, for example, the flows in the orthogonal $(\phi=\pi / 2)$ and back-to-back $(\phi=\pi)$ azimuthal directions, one gets

$$
C^{q \bar{q}}\left(\eta_{34}, \frac{\pi}{2}\right)=1 \quad, \quad C^{q \bar{q}}(0, \pi)=\frac{N_{c}^{2}-2}{2\left(N_{c}^{2}-1\right)}=\frac{7}{16} .
$$

This implies that for $\phi=\pi / 2$ the hadronic multiplicities are uncorrelated, while for $\phi=\pi$ there is destructive interference, of the same magnitude as the drag effect in symmetric jets given by (9.38). Thus, measurements of hadronic flow correlations in the orthogonal and back-to-back azimuthal directions should demonstrate the same type of color coherence as the drag effect without requiring the selection of a three-jet event sample. Notice, that contrary to the string/drag effect in three-jet events here there are no reasons for the conventional string model to mimic this correlation pattern 
without including multiple gluon bremsstrahlung. An analysis of color flow along the above lines may be performed for other correlations as well. For example, study of asymmetry of azimuthal jet profiles may help to visualize $1 / N_{c}^{2}$ effects that are typically neglected in algorithmic shower models.

An interesting manifestation of the QCD wave nature of hadronic flows arises from the double-inclusive correlations $d^{2} N / d \Omega_{\alpha} d \Omega_{\beta}(\alpha, \beta$ denote the interjet valleys) of the interjet flows in $q \bar{q} g$ events. Here one faces such tiny effects as the mutual influence of different $q \bar{q}$ antennae. As a consequence

$$
\frac{d^{2} N / d \Omega_{(g q)} d \Omega_{(g \bar{q})}}{d^{2} N / d \Omega_{(q \bar{q})} d \Omega_{(g \bar{q})}} \equiv r_{2} \quad<\frac{d N / d \Omega_{(g q)}}{d N / d \Omega_{(q \bar{q})}} \equiv r_{1}
$$

(detailed analytic formulae may be found in Ref. [2]). 
Problem 9.1 Using the LLA formulae of Subsection 1.3.4 derive the mean momenta $\left\langle z^{B}\right\rangle_{A}$ carried by gluons and quarks defined by (9.10).

\section{Solution:}

According to the definition (1.93) of the parton spectra in the moment representation

$$
\left\langle z^{B}\right\rangle_{A}=D(j=2, \xi) \quad \text { with } \quad \xi=\xi\left(E \Theta_{0}\right)-\xi(E \Theta) .
$$

Equation (1.104), (1.105) then give

$$
\begin{array}{clrl}
\nu_{F}(2)=-\frac{8}{3} C_{F}, & \nu_{G}(2)=-\frac{2}{3} n_{f}, & \Phi_{F}^{G}(2)=\frac{8}{3} C_{F}, & \Phi_{G}^{F}(2)=\frac{1}{3} ; \\
\nu_{-}(2)=-\left(\frac{8}{3} C_{F}+\frac{2}{3} n_{f}\right) \equiv-\gamma, & \nu_{+}(2)=0 .
\end{array}
$$

Constructing the residues (1.107)-(1.110) one finally arrives at

$$
\begin{array}{ll}
\left\langle z^{q}\right\rangle_{g}=\beta \cdot\left(1-e^{-\gamma \xi}\right), & \left\langle z^{g}\right\rangle_{g}=\alpha+\beta \cdot e^{-\gamma \xi} ; \\
\left\langle z^{g}\right\rangle_{q}=\alpha \cdot\left(1-e^{-\gamma \xi}\right), & \left\langle z^{q}\right\rangle_{q}=\beta+\alpha \cdot e^{-\gamma \xi} \equiv D^{v a l}+D^{\text {sea }}
\end{array}
$$

with $\alpha, \beta$ and $\gamma$ defined by (9.13).

Problem 9.2 Check that the integration of (9.18) over the solid angle reproduces the relation

$$
N_{e^{+} e^{-}}(W)=2 N_{q}(E) .
$$

Problem 9.3 Check the relation (9.36).

Problem 9.4 Derive asymptotic prediction (9.38) for the drag effect.

Problem 9.5 Taking the expression (9.34) for the $q \bar{q} g$ antenna pattern derive the ratio of the particle flow in the direction, transversal to the scattering plane, to the flow between quarks (9.39) for symmetric q $\bar{q} g$ events. 


\section{Bibliography}

1. A. Bassetto, M. Ciafaloni, and G. Marchesini. Phys. Rep., C100:201, 1983.

2. Yu.L. Dokshitzer, V.A. Khoze and S.I. Troyan. Coherence and physics of QCD jets. In A.H. Mueller, editor, Perturbative QCD, page 241. World Scientific, Singapore, 1989.

3. Yu.L. Dokshitzer, V.A. Khoze, A.H. Mueller and S.I. Troyan. Rev. Mod. Phys., 60:373, 1988.

4. JADE Collaboration, A. Petersen. In J. Tran Than Van, editor, Elementary Constituents and Hadronic Structure, page 505. Editions Frontières, Dreux, 1980.

5. JADE Collaboration, W. Bartel et al. Phys. Lett., B101:129, 1981. $Z$ Z Phys., C21:37, 1983. Phys. Lett., B134:275, 1984.

6. TPC $/ 2 \gamma$ Collaboration, H. Aihara et al. Z. Phys., C28:31, 1985.

7. TASSO Collaboration, M. Althoff et al. Z. Phys., C29:29, 1985.

8. B. Andersson, G. Gustafson and T. Söstrand. Phys. Lett., 94B:211, 1980.

9. Ya.I. Azimov, Yu.L. Dokshitzer, V.A. Khoze and S.I. Troyan. Phys. Lett., 165B:147, 1985. Sov. J. Nucl. Phys., 43:95, 1986.

10. W. de Boer. preprint CERN-PPE / 90-161, 1990.

11. OPAL Collaboration, M.Z. Akrawy et al. CERN-PPE / 91-31, 1991.

12. S.D. Ellis, Z. Künszt and D.E. Soper. Phys. Rev. Lett., 62:726, 1989.

13. S.D. Ellis. In R. Slansky and G. West, editors, Proceedings of the 1987 Theoretical Advanced Study Institute in Elementary Particle Physics, Santa Fe, New Mexico, page 274. World Scientific, Singapore, 1988. 
14. Z. Künst, P. Nason, G. Marchesini and B.R. Webber. QCD. In G. Altarelli, R. Kleiss, and C. Verzegnassi, editors, Proceedings of the Workshop on $Z$ physics at LEP, CERN Report 89-08, volume 1, page 373, 1989.

15. Yu.L. Dokshitzer, V.A. Khoze, G. Marchesini and B.R. Webber. Phys. Lett., 245:243, 1990.

16. Yu.L. Dokshitzer, V.A. Khoze and S.I. Troyan. In M. Derrick, editor, Proceedings of the 6th Int. Conf. on Physics in Collisions, page 417. World Scientific, Singapore, 1987.

17. C. Basham, L. Brown, S. Ellis and S. Love. Phys. Rev. Lett., 41:1585, 1978.

18. Yu.L. Dokshitzer, D.I. Dyakonov and S.I. Troyan. Phys. Lett., 78B:290, 1978.

19. Yu.L. Dokshitzer, D.I. Dyakonov and S.I. Troyan. Phys. Rep., 58:270, 1980.

20. B.R. Webber and P. Rakow. Nucl. Phys., B187:254, 1981.

21. W. Hofmann. In W. Bartel and R. Rückl, editors, Proc. of the 1987 Int. Symp. on Lepton and Photon Interactions at High Energies, page 671. North Holland, Amsterdam, 1987.

22. TPC $/ 2 \gamma$ Collaboration, H. Aihara et al. Phys. Rev. Lett., 57:945, 1986.

23. MARKII Collaboration, P.D. Sheldon et al. Phys. Rev. Lett., 57:1398, 1986.

24. S.J. Brodsky and J.F. Gunion. Phys. Rev. Lett., 37:402, 1976.

25. M. Bosman et al. Heavy Flavours. In G. Altarelli, R. Kleiss, and C. Verzegnassi, editors, Proceedings of the Workshop on $Z$ physics at LEP, CERN Report 89-08, volume 1, page 267, 1989. 
26. L. Lönnblad, C. Peterson and T. Rögnvaldsson. Phys. Rev. Lett., 10:1321, 1990.

27. B. Andersson, G. Gustafson, G. Ingelman and T. Söstrand. Phys. Rep., 97:31, 1983.

28. V.A. Khoze and L. Lönnblad. Phys. Lett., 241B:123, 1990.

29. T. Sjöstrand. CERN-TH 5902, 1990. 


\section{Chapter 10}

\section{Collective Color Phenomena in Hadronic Hard Scattering}

\section{Contents}

10.1 QCD Coherence in High $-p$, Collisions . . . . . . . 322

10.2 Color Transfer and Particle Flows . . . . . . . . 324

10.3 Two-Jet Production at Large $p$

10.4 Prompt $\gamma, W, Z$ Production at Large $p . \ldots 334$

10.5 Color Structure of Heavy Quark Production . . 339

10.6 Azimuthal Asvmmetrv of QCD Jets . . . . . . . . 343

10.6.1 Jet Asvmmetrv in $e^{+} e^{-}$annihilation . . . . . . . . 344

10.6.2 Jet Asvmmetrv in hadron scattering . . . . . . . 348

10.7 Lessons . . . . . . . . . . . . . . . . 353

Appendix: Radiation in Parton-Parton Scattering . . 358 


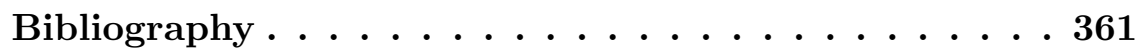

In other types of hard collisions color coherence also leads to a rich diversity of collective drag effects (for reviews see Refs. [1 [5]). In this Section we discuss soft particle production accompanying high- $p_{\perp}$ hadronic collisions. Our task here is to describe the characteristic properties of final states in different types of hard scattering processes and to study, in particular, the interference pattern which emerges when soft gluons are emitted by several sources in a coherent manner.

\subsection{Implications of QCD Coherence to High $-p_{\perp}$ Collisions}

\section{Virtues of High $-p_{\perp}$ Processes}

1. A diversity of hard interactions as small distances. Varying the experimental conditions (triggers) one may extract the dominant subprocess and turn from one subprocess to another.

2. Dependence of length and height of the "plateau" in hadron spectra on the different parameters: the length is determined by the total energy of the collision, and the height and the plateau structure depend on the hardness of the process governed, as a rule, by the transverse energy-momentum transfer. Thus, information becomes available that is inaccessible in $e^{+} e^{-}$annihilation where both energy and hardness were given by the value of $W$. 
3. These reactions are the best source of high energy gluon jets.

4. Recall that just in high- $p_{\perp}$ hadronic collisions the largest possible energies (hardness) will be reached.

5. Finally, detailed studies of such processes are necessary for designing future experiments and analysis of their data, attempting, in particular, to find new heavy objects.

There is a flip side of this coin. For hadronic collisions the underlying physics is more complicated, than, say, for $e^{+} e^{-}$annihilation (e.g., because of the presence of colored constituents in both the initial and final states), and the energy range of applicability of the PT approach is now less clear. However, the nature of jets, based on the dominant role of the QCD bremsstrahlung processes, is the same for both reactions. Therefore, at least at the energies of future hadronic colliders, the main physical phenomena in jet physics and characteristics of the final states should also be under the control of PT dynamics. Presumably at LHC-SSC energies color coherence effects should be well distinguishable from the minimum bias background. Coherent drag phenomena could provide a valuable tool for extracting and studying manifestations of New Physics.

At sufficiently high energies it is the color structure of the parton collision that determines the hadronic structure of high- $p_{\perp}$ events. In the framework of the LPHD concept the QCD radiation pattern is believed to survive the hadronization stage, so that observed hadrons should "remember" the color topology of the basic hard scattering of partons. 


\subsection{Color Transfer and Particle Flows}

In course of a hard interaction color is transferred abruptly from one parton to another. For example, the colliding quarks 1 and 2 in the color states $i$, $k$ are simply "recharged" in the large- $N_{c}$ approximation after the $t$-channel gluon exchange

$$
1_{i}+2_{k} \rightarrow 1_{j}^{\prime}+2_{m}^{\prime} \quad: \quad t_{j i}^{a} t_{m k}^{a}=\frac{1}{2} \delta_{i m} \delta_{j k}-\frac{1}{2 N_{c}} \delta_{i j} \delta_{m k}
$$

The parton-parton scattering acts here as a color antenna. Gluon bremsstrahlung associated with the incoming and the outgoing partons leads to the formation of jets of hadrons around the directions of these four colored emitters.

To demonstrate, how the interjet coherence connects the structure of hadronic accompaniment with the $t$-channel color transfer, let us consider high- $p_{\perp}$ scattering of energetic partons $A$ and $B\left(E_{A} \sim E_{B} \sim \sqrt{s}\right)$ at relatively small angles $\Theta_{s} \approx p_{\perp} / E \ll 1$, as shown in Fig. 10.1.

The hardness of the process is determined by the momentum transfer $p_{\perp} \approx$ $\sqrt{-\hat{t}}$, which naturally restricts the transverse momenta of the accompanying gluon bremsstrahlung $\ell_{\perp}<p_{\perp}$ and, so the development of partonic cascades.

In the structure of the final hadronic system three regions may be separated. Two of them adjoin the fragmentation regions of the colliding particles and occupy the intervals of the pseudorapidity $\eta$ :

$$
\Delta \eta=\ln \frac{p_{\perp}}{\Lambda}, \quad \eta=\frac{1}{2} \ln \frac{1+\cos \Theta}{1-\cos \Theta} .
$$

The hadronic spectrum in each of these intervals ("extended fragmentation regions") is saturated with the particles from the two bremsstrahlung cones of the incoming and the scattered partons, and so results from an incoherent 


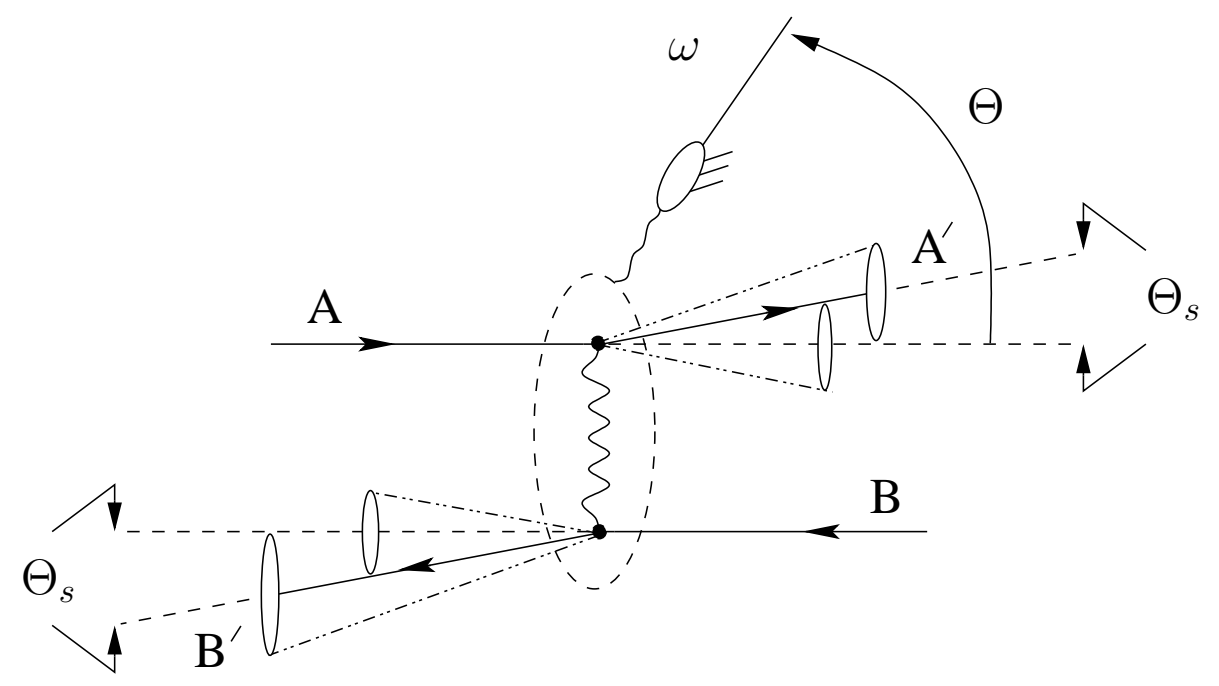

Figure 10.1: Soft gluon bremsstrahlung accompanying small angle scattering of partons $A+B \rightarrow A^{\prime}+B^{\prime}$. $\Theta_{s} \ll 1$ is the scattering angle.

sum of two jets with angular opening $\Theta=\Theta_{s}$. The height of the distribution is determined, roughly speaking, by the sum of parton "color charges" $C_{A}+C_{A^{\prime}}$ and $C_{B}+C_{C^{\prime}}$ respectively. In the central region

$$
|\eta|<\eta\left(\Theta_{s}\right) \approx \ln \frac{\sqrt{s}}{p_{\perp}} \approx \frac{E}{p_{\perp}}
$$

(final particle angles larger than the scattering angle $\Theta_{s}$ ), the incoming and the scattered partons radiate coherently, and, as a result, the hadron density is determined by the color charge $C_{t}$ of the $t$-channel exchange $\left(C_{t}=N_{c}\right.$ for gluon exchange, $C_{t}=C_{F}$ for quark exchange). Since in the given kinematics $(-\hat{t} \ll \hat{s})$ gluon exchange dominates, we conclude that in the region (10.1) 
hadronic spectrum is determined by the "color strength" of the gluon current $C_{t}=N_{c}$, and what is of importance, it becomes universal, independent of the nature of scattered partons ( $A, B$ being light or heavy quarks or gluons).

\section{Rapidity Plateau}

How should the rapidity distribution of hadrons accompanying the high- $p_{\perp}$ scattering process look like? We are interested now in the kinematical region of large angles (10.1) where the registered particles originate not from bremsstrahlung cones initiated by incoming and scattered partons, but are caused by their common coherent radiation due to $t$-channel color transfer. Let us take such a particle with transverse momentum $k_{\perp}$. It is relatively soft partons with

$$
R^{-1} \ll k_{\perp} \ll \sqrt{-t}
$$

which determine the multiplicity flow at fixed $\Theta$. Therefore a given particle will normally be an offspring of a subjet with momentum $\ell$

$$
k_{\perp} \ll \ell_{\perp} \ll \sqrt{-t} .
$$

This subjet as a whole can be said to have, within logarithmic accuracy, the same emission angle $\Theta$, with the ratio $k_{\perp} / \ell_{\perp}=x$ standing for the jet momentum fraction carried by $k$. Since the parent parton $\ell$ will be predominantly a soft gluon, the resulting spectrum can be represented by the formula

$$
\frac{d \sigma}{\sigma d \eta d k_{\perp}} \approx 4 C_{t} \int_{k_{\perp}}^{p_{\perp}} \frac{d \ell_{\perp}}{\ell_{\perp}^{2}} \frac{\alpha_{s}\left(\ell_{\perp}^{2}\right)}{2 \pi} \bar{D}_{g}\left(\frac{k_{\perp}}{\ell_{\perp}}, \ln \frac{\ell_{\perp}}{\Lambda}\right) .
$$

Here $x \bar{D}_{g}(x, \ln Q / \Lambda)$ is the standard distribution of particles with energy fraction $x$ in a gluon jet, for which the product of energy and opening angle equals $Q$. 
Integrating over $k_{\perp}$ to get the inclusive rapidity spectrum, we obtain

$$
f\left(\eta, \ln p_{\perp}\right) \equiv \frac{d \sigma}{\sigma d \eta}=\int^{p_{\perp}} \frac{d \ell_{\perp}}{\ell_{\perp}} 4 C_{t} \frac{\alpha_{s}\left(\ell_{\perp}^{2}\right)}{2 \pi} \int_{0}^{1} d x \bar{D}_{g}\left(x, \ln \frac{\ell_{\perp}}{\Lambda}\right) .
$$

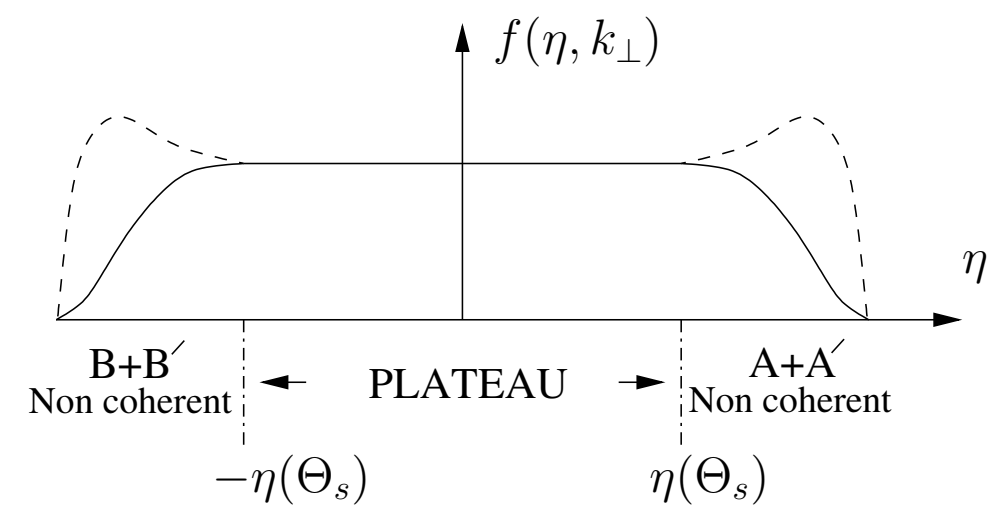

Figure 10.2: Universal rapidity plateau at $|\eta|<\ln 1 / \Theta_{s}$. Solid and dashed lines illustrate schematically the difference between $q q$ and $g g$ scattering.

This expression shows that the yield of hadrons in the rapidity interval $|\eta(\Theta)|<\eta\left(\Theta_{s}\right) \approx \ln E / p_{\perp}$ does not depend on the rapidity, so that a flat plateau distribution emerges (see Fig. 10.2). The plateau height is proportional to the $t$-channel "color charge" $C_{t}$ and increases with the hardness scale of the scattering process. Comparing (10.2) with the DLA equation for the jet multiplicity (5.47), one arrives at the formula which is familiar already to us:

$$
f\left(\ln p_{\perp}\right) \approx \frac{C_{t}}{N_{c}} N_{g}^{\prime}\left(\ln \frac{p_{\perp}}{\Lambda}\right) .
$$

Color interference between jets seems to become a phenomenon of large potential value as a new additional tool for discriminating between hard processes. 
For example, reconstruction of the antenna pattern from the effects of particle drag may help to visualize the production mechanisms of new heavy objects - the Higgs boson $H$, new quarks and leptons, supersymmetric particles etc. Most of these objects produce hadronic jets, and the configurations of interjet particles should differ from the conventional QCD processes. An interesting example comes from the comparison of the production of a colorless object via $g g$ of $q \bar{q}$ collisions. Here, if the hard kinematics is the same, the heights of the accompanying plateau should differ by a factor of $N_{c} / C_{F}$.

\subsection{Two-Jet Production at Large $p_{\perp}$}

Hadronic high- $p_{\perp}$ processes are rich in the collective drag phenomena. Let us consider, e.g., the topology of events, resulting from the $q q$ scattering

$$
-\hat{t} \sim \hat{s}=x_{1} x_{2} s \quad\left(x_{1}, x_{2} \sim 1\right) .
$$

In this case the two crossing processes shown in Fig. 10.3(a) and 10.3(b) have approximately equal probabilities. However each of them has its own color topology, and therefore specific particle flows, as schematically shown in Fig. 10.3 (c) and (d).

For the subprocess of Fig. 10.3)(a) the soft particle radiation pattern is

$$
\begin{gathered}
\frac{4 \pi d N^{q_{1} q_{2}}}{d \Omega_{\vec{n}}}=\frac{C_{F}}{N_{c}} N_{g}^{\prime}\left(\ln \frac{E}{\Lambda}\right) . \\
\left\{\widehat{(14)}+\widehat{(23)}+\frac{1}{2 N_{c} C_{F}}(2[\widehat{(12)}+\widehat{(34)}]-\widehat{(14)}-\widehat{(23)}-\widehat{(13)}-\widehat{(24)})\right\} .
\end{gathered}
$$



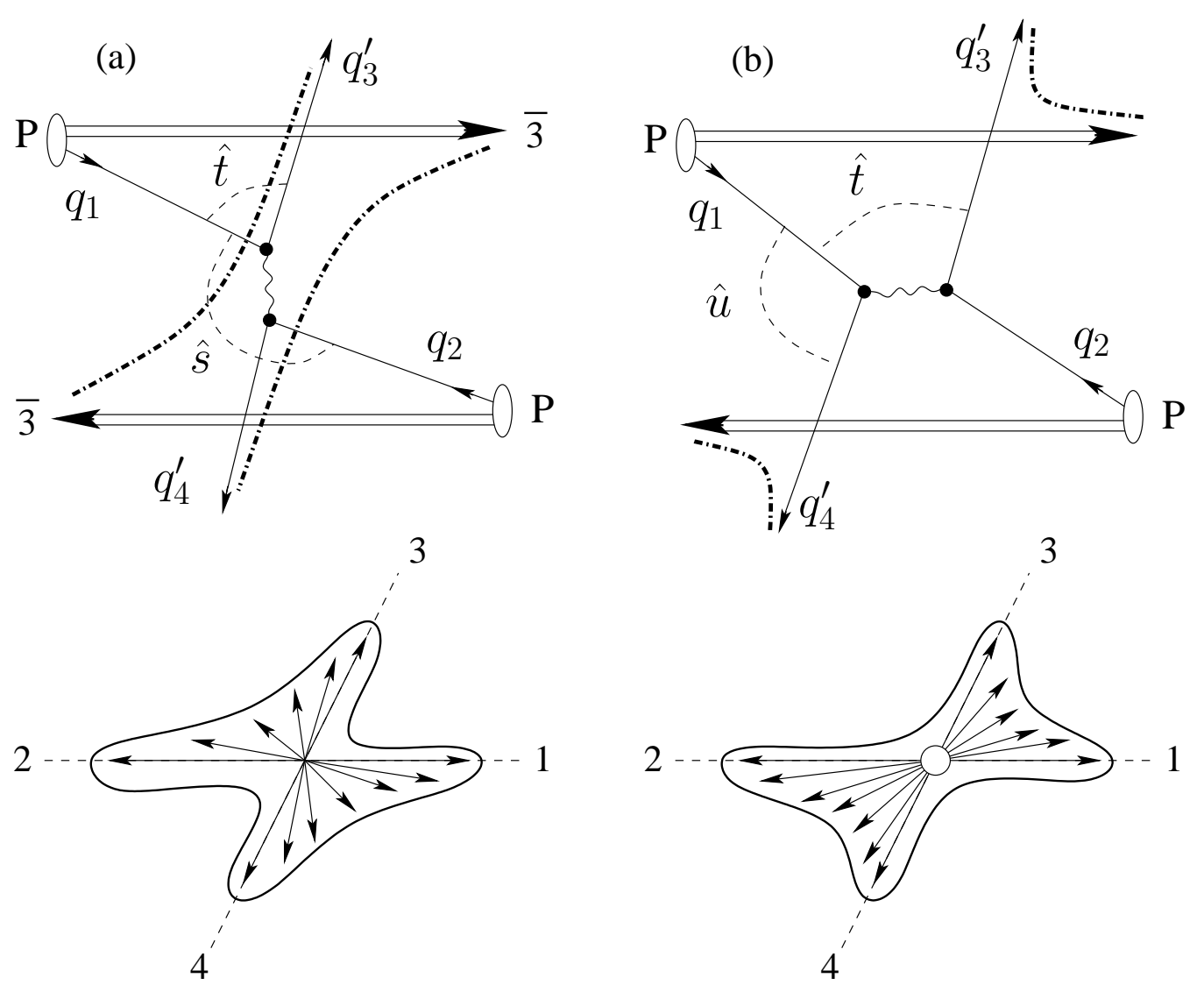

Figure 10.3: Color antennae for two crossing high- $p_{\perp} q q$ scattering processes and the drawings of the corresponding particle flows. 


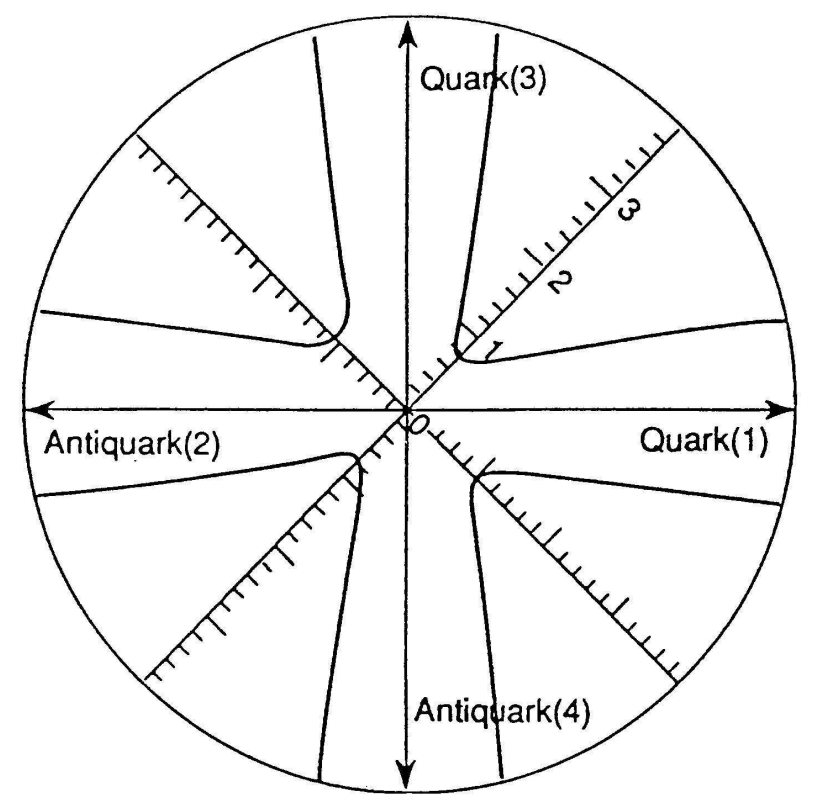

Figure 10.4: Polar plot ${ }^{[3]}$ showing the antenna pattern function $P(-1 / 2,-1 / 2, \Theta, 0)$ versus $\Theta$ for the identical quark process $q\left(p_{1}\right)+\bar{q}\left(p_{2}\right) \rightarrow$ $q\left(p_{3}\right)+\bar{q}\left(p_{4}\right)$. The magnitude of $P$ is given by the radial distance of the curves from the origin. The plot has been cut off at $P=4$, so the singularities in the forward regions are not displayed. 
In full analogy with the discussions of the drag effect in the previous Section one may say that the leading contribution (the first term in (10.3) ) has the structure of the sum of two independent $q \bar{q}$-antennae $\widehat{(14)}$ and $\widehat{(23)}$. This fact also can be mimicked by means of the topological picture of the Lund string model. Let us emphasize that in our case to each contribution (single antenna) a dynamical distribution corresponds which takes into account the effects of cascade multiplication. Furthermore, the perturbative approach permits one to control not only the leading color contribution, but also the $O\left(1 / N_{c}\right)$ corrections. The antenna patterns for the $q \bar{q}(q)$ scattering subprocesses for the case of different and identical quark flavors are presented in the Appendix.

For illustration we show in Fig. 10.4 the antenna pattern function $P$

$$
\pi \frac{d N}{d \Omega_{\vec{n}}}=P\left(\frac{t}{s}, \frac{u}{s} ; \Theta, \phi\right) N_{g}^{\prime}
$$

for the case of identical flavor scattering

$$
q\left(p_{1}\right)+\bar{q}\left(p_{2}\right) \rightarrow q\left(p_{3}\right)+\bar{q}\left(p_{4}\right)
$$

at $90^{\circ}$ in the parton center of mass. Here $s, t, u$ define the kinematical variables of the $2 \rightarrow 2$ hard scattering:

$$
s=\left(p_{1}+p_{2}\right)^{2}, \quad t=\left(p_{1}-p_{3}\right)^{2}, \quad u=\left(p_{1}-p_{4}\right)^{2} .
$$

The explicit formulae describing antenna patterns for the $q g$ and $g g$ scattering are more complicated than for $q q(q \bar{q})$ case (see Refs. [16] for details).

For example, for the $g g$ scattering events with small scattering angle $\Theta_{s} \ll 1$, when one-gluon exchange dominates in $t$-channel, two color configurations of the participating gluons contribute, as shown in Fig 10.5 Here 


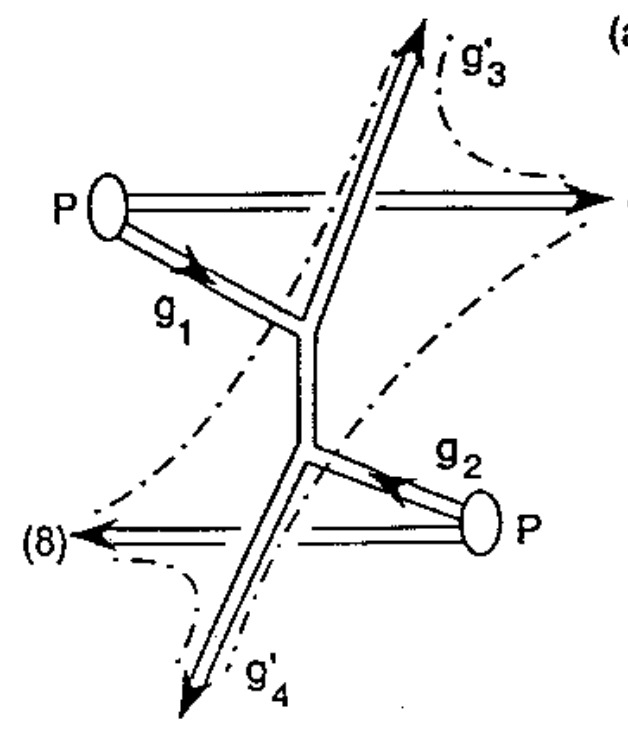

(a)

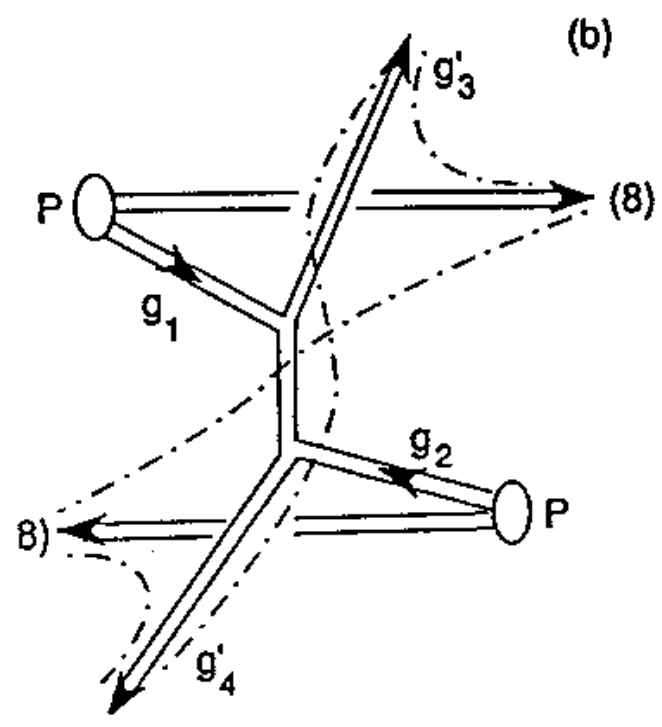

Figure 10.5: Color antennae for hard scattering $g_{1}+g_{2} \rightarrow g_{3}+g_{4}$ in the case when $t$-channel gluon exchange dominates $\left(\Theta_{s} \ll 1\right)$.

the associated soft radiation reads (see Appendix)

$$
8 \pi \frac{d N^{g_{1} g_{2}}}{d \Omega_{\vec{n}}}=\left\{\widehat{(13)}+\widehat{(24)}+\frac{1}{2}[\widehat{(12)}+\widehat{(34)}+\widehat{(14)}+\widehat{(23)}]\right\} \cdot N_{g}^{\prime}\left(\ln \frac{E}{\Lambda}\right)
$$

Pattern function (10.4) for $90^{\circ} \mathrm{gg}$-scattering is displayed in Fig.10.6)(a). Noteworthy to mention a symmetry between four quadrants. A different situation occurs in the case of $q g$-scattering shown in Fig. 10.6(b). A large asymmetry 

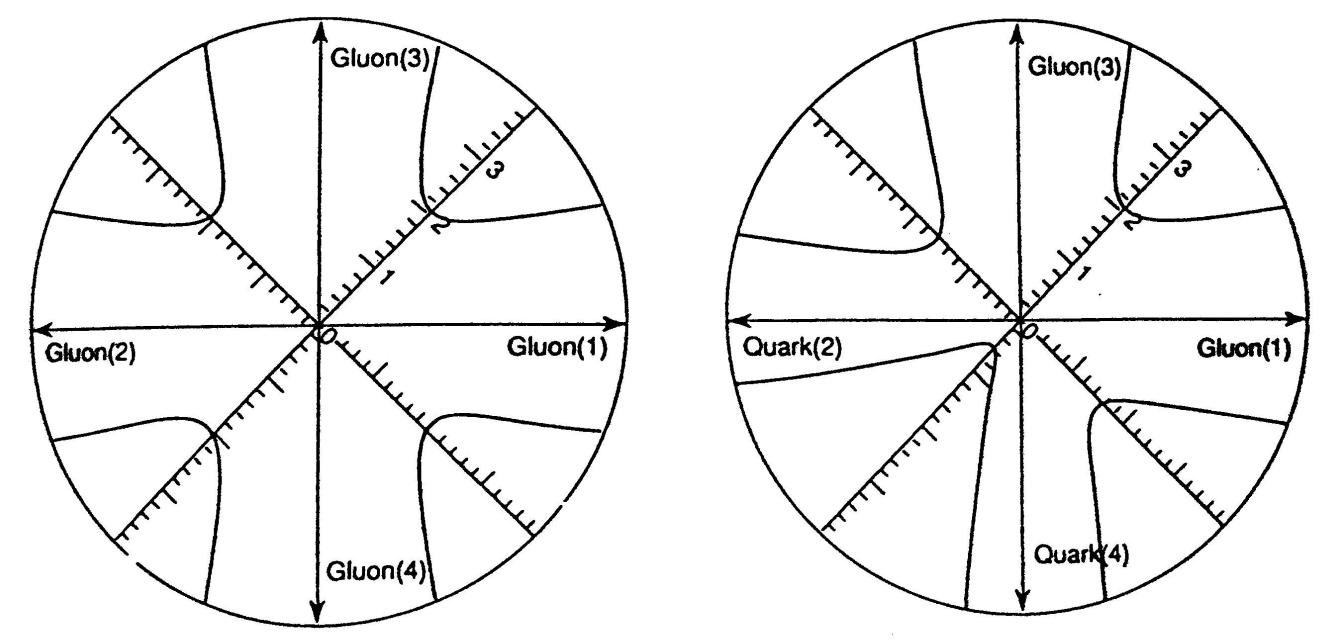

Figure 10.6: Polar plots 3 showing the antenna patterns $P(-1 / 2,-1 / 2, \Theta, 0)$ for $\quad(\mathrm{a}): \quad g_{1}+g_{2} \rightarrow g_{3}+g_{4}, \quad(\mathrm{~b}): \quad g_{1}+q_{2} \rightarrow g_{3}+q_{4}$

of about a factor of four between $g g$ and $q q$ quadrants in clearly seen here. If detectable, this asymmetry would allow the separation of $g$ - and $q$-jets on an event-by-event basis. By moving to large rapidity one can select events in which the fraction of the longitudinal momentum carried by one of the partons is very large and the other is very small thus enriching contribution from the $q g$-scattering. 


\subsection{Prompt $\gamma, W, Z$ Production at Large $p_{\perp}$}

A drag effect, very similar to 3 -jet events, can be studied in high- $p_{\perp}$ processes, such as $\gamma \cdot \mu^{+} \mu^{-}$pair, $W, Z$ production, where a colorless object is used as a trigger 27.

Consider the three jet production

$$
p+\bar{p} \rightarrow \gamma\left(p_{\perp}\right)+\text { jet }_{1}+\text { jet }_{2}+\text { jet }_{3} .
$$
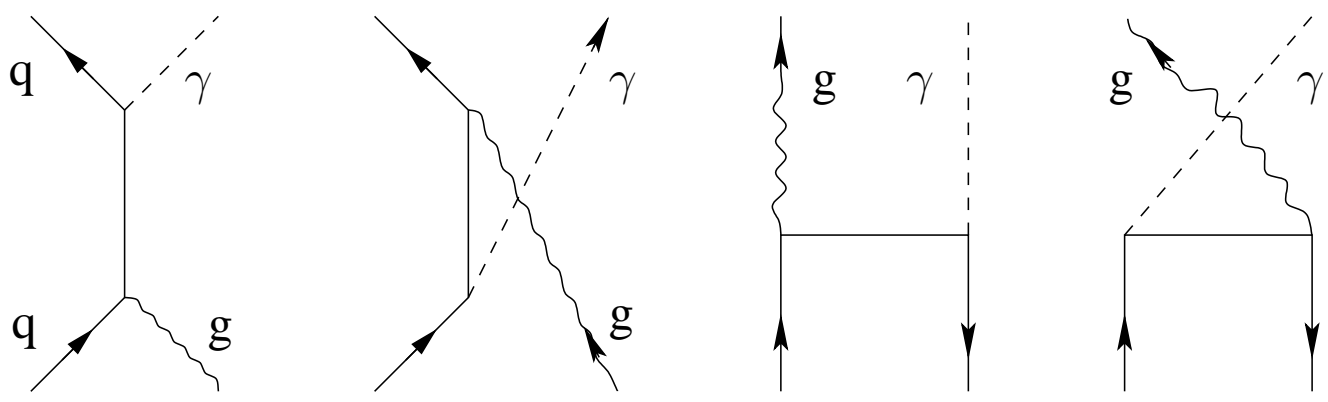

Figure 10.7: Hard scattering graphs leading to $\gamma+$ jet production.

The basic graphs describing the process are shown in Fig. 10.7. We shall argue in a moment that $g q \rightarrow \gamma q$ dominates $q \bar{q} \rightarrow \gamma g$. Keeping only the hard Compton scattering graph, the cross section for a hard photon of transverse momentum $p_{\perp}$ corresponding to $90^{\circ}$ scattering in the parton $\mathrm{cms}$ reads (see, e.g., reviews [58)

$$
\frac{d \sigma}{d p_{\perp}^{2} d \cos \Theta}=\sum_{q} e_{q}^{2} \int d y x_{q} D_{p}^{q}\left(x_{q}, p_{\perp}^{2}\right) x_{g} D_{\bar{p}}^{g}\left(x_{g}, p_{\perp}^{2}\right) \frac{d \sigma}{d p_{\perp}^{2}}+(p \leftrightarrow \bar{p}) .
$$


In the above $x_{g} \cdot x_{q} \cdot s=4 p_{\perp}^{2}$ and $y=y_{1}+y_{2}$ with $y_{1}$ and $y_{2}$ the rapidities of the outgoing $\gamma$ and gluon jet respectively, $e_{q}$ the quark electric charge. Then

$$
\frac{d \sigma}{d p_{\perp}^{2} d y d \cos \Theta}=\sum_{q} e_{q}^{2} \frac{5 \pi \alpha \cdot \alpha_{s}}{96 p_{\perp}^{4}} x_{q} D_{p}^{q}\left(x_{q}, p_{\perp}^{2}\right) x_{g} D_{\bar{p}}^{g}\left(x_{g}, p_{\perp}^{2}\right)+(p \leftrightarrow \bar{p}) .
$$

The corresponding formula for the annihilation process $q \bar{q} \rightarrow \gamma g$ is

$$
\frac{d \sigma}{d p_{\perp}^{2} d y d \cos \Theta}=\sum_{q} e_{q}^{2} \frac{\pi \alpha \cdot \alpha_{s}}{9 p_{\perp}^{4}} x_{q} D_{p}^{q}\left(x_{q}, p_{\perp}^{2}\right) x_{\bar{q}} D_{\bar{p}}^{\bar{q}}\left(x_{\bar{q}}, p_{\perp}^{2}\right)+(p \leftrightarrow \bar{p}) .
$$

When $x_{\bar{q}}\left(x_{g}\right) \lesssim 0.1$ the Compton process dominates and one can neglect the annihilation contribution.
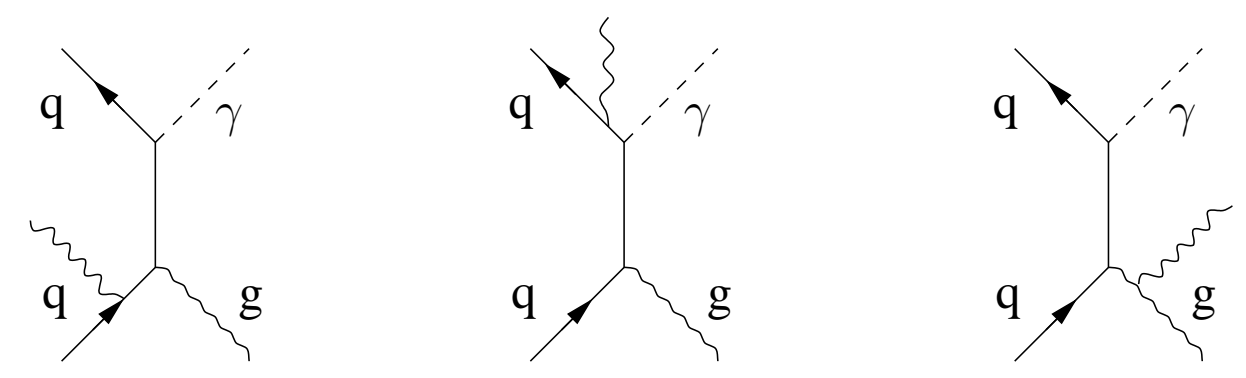

Figure 10.8: Soft gluon emission from the hard scattering graphs of Fig. 10.7

The picture of the soft accompanying emission is schematically illustrated in Fig. 10.8. The formula for radiation associated with the hard scattering here has the same form as (9.34),

$$
\frac{8 \pi d N}{d \Omega_{\vec{n}}}=\left[\widehat{(23)}+\widehat{(21)}-\frac{1}{N_{c}^{2}} \widehat{(13)}\right] N_{g}^{\prime}\left(Y_{m}\right)
$$


with $\vec{n}_{i}$ the direction of parton $i$, as shown in Fig. 10.9. The variable $Y_{m}=$ $\ln E \Theta_{m} / \Lambda$ governs the evolution of a jet with energy $E$ and opening angle $\Theta_{m}=\min \left\{\Theta_{1}, \Theta_{2}, \Theta_{3}\right\}, \quad \cos \Theta_{i}=\left(\vec{n} \vec{n}_{i}\right)$.

As discussed earlier in Section [7.9] a reasonable phenomenology was done for $\pi^{ \pm}$production, taking the limiting MLLA formulae for multiplicity of pions in a gluon jet, $N_{g}$. To illustrate the structure of the coherence pattern we present below results of evaluating (10.7) for final state pions projected onto the plane of the hard scattering and at angles midway between the parton directions labeled by $A, B, C$ and $D$ in Fig. 10.9.

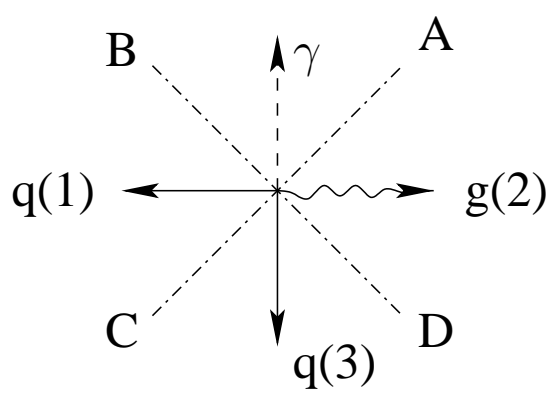

Figure 10.9: Kinematics of $90^{0}$ scattering in the hard process $q\left(p_{1}\right)+g\left(p_{2}\right) \rightarrow$ $\gamma+q\left(p_{3}\right)$.

Thus, $d N^{A} / d \vec{n}$ corresponds to the density of pions in the scattering plane between directions of incoming gluon and outgoing photon. In Table 10.1 are shown the values of pion multiplicities in four $30^{\circ}$ sectors for different values of $E_{\perp} / \Lambda$.

As is easily seen, particle production is largest between the directions of the incoming gluon and outgoing quark, but $\sim 2.8$ times smaller between the directions of the incoming quark and the outgoing quark. So, just as in the 


\begin{tabular}{|c||c|c|c|c|}
\hline$E_{\perp} / \Lambda$ & 60 & 200 & $10^{3}$ & $10^{4}$ \\
\hline \hline $\mathrm{A}$ & 0.62 & 1.04 & 1.94 & 4.3 \\
\hline $\mathrm{B}$ & 0.52 & 0.88 & 1.57 & 3.4 \\
\hline $\mathrm{C}$ & 0.51 & 0.87 & 1.61 & 3.5 \\
\hline $\mathrm{D}$ & 1.47 & 2.44 & 4.47 & 9.6 \\
\hline
\end{tabular}

Table 10.1: The energy rise of pion multiplicities in the interjet $30^{\circ}$ sectors.

$e^{+} e^{-} \rightarrow q \bar{q} g$ reaction, the drag of hadrons is predicted in the direction of the gluon jet. This leads to an azimuthal asymmetry of particles, which can be seen by looking end on at the struck $q$-jet, see for details Section 10.6.

A process very similar to hard $\gamma$ production is that of high- $p_{\perp} W$ production. Here the cross section is 518

$$
\frac{d \sigma}{d y_{1} d y_{2} d p_{\perp}^{2}}=\sum_{i j} x_{1} D_{p}^{i}\left(x_{1}, p_{\perp}^{2}\right) x_{2} D_{\bar{p}}^{i}\left(x_{2}, p_{\perp}^{2}\right) \frac{d \hat{\sigma}_{i j}}{d \hat{t}},
$$

with $i, j$ a sum over partons and $d \hat{\sigma}_{i j} / d \hat{t}$ the hard scattering cross section $i+j \rightarrow W+$ parton. $y_{1}$ and $y_{2}$ are the rapidities of the $W$ and the recoil parton while $p_{\perp}$ is the transverse momentum of the $W$.

At large $p_{\perp}$ there are two fundamental processes which contribute:

$$
\begin{aligned}
& q\left(p_{1}\right)+\bar{q}\left(p_{2}\right) \rightarrow W\left(p_{4}\right)+g\left(p_{3}\right), \\
& q\left(p_{1}\right)+g\left(p_{2}\right) \rightarrow W\left(p_{4}\right)+q\left(p_{3}\right) .
\end{aligned}
$$




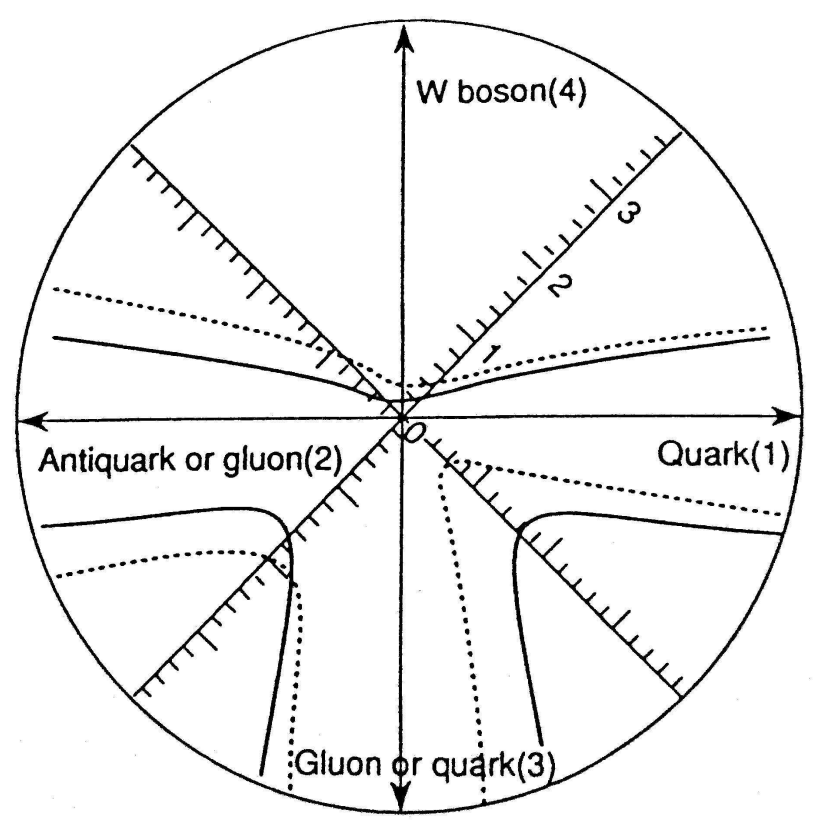

Figure 10.10: Polar plot 3 showing the antenna pattern $P\left(-\frac{1}{2},-\frac{1}{2} ; \Theta, 0\right)$ versus $\Theta$ for the process $q\left(p_{1}\right)+\bar{q}\left(p_{2}\right) \rightarrow W\left(p_{4}\right)+g\left(p_{3}\right)$, (solid line) and $q\left(p_{1}\right)+$ $g\left(p_{2}\right) \rightarrow W\left(p_{4}\right)+q\left(p_{3}\right)$, (dashed line) for $90^{\circ}$ scattering in the parton $\mathrm{cms}$. 
The basic cross sections are

$$
\begin{aligned}
\frac{d \sigma_{u \bar{d}}}{d t} & =\frac{2 \pi \alpha \cdot \alpha_{s}}{9 \sin ^{2} \Theta_{W}} \frac{\left(t-M_{W}^{2}\right)^{2}+\left(u-M_{W}^{2}\right)^{2}}{s^{2} t u}, \\
\frac{d \sigma_{u g}}{d t} & =\frac{\pi \alpha \cdot \alpha_{s}}{12 \sin ^{2} \Theta_{W}} \frac{s^{2}+u^{2}+2 M_{W}^{2} t}{-s^{3} u}+(t \leftrightarrow u) .
\end{aligned}
$$

The associated soft gluon spectrum is quite different in the Compton and annihilation processes. For $q g \rightarrow W q$ (10.8b) it it given by (10.7) while for $q \bar{q} \rightarrow W g$ (10.8a $)$ the antenna pattern can be obtained by the interchange $(2 \leftrightarrow 3)$, see Ref. [2].

Fig. 10.10 illustrates the antenna pattern function $P$ of (10.4) for the processes (10.8) at $90^{\circ}$ in the patron $\mathrm{cms}$. One can easily see the essential qualitative difference between the two mechanisms of the $W$-boson production. From the process $(i)$ the particle density between initial quark (1) and final gluon (3) is equal to that between initial quark (2) and (3). Meantime, the radiation due to $(i i)$ which is large between the directions of the incoming gluon (2) and the outgoing quark (3), appears to be much smaller between the directions of the incoming quark (1) and the outgoing one (3), $c f$. Table 10.1 . Therefore one could suggest that it might be possible to distinguish whether a $W$ is produced by process $(i)$ or $(i i)$ on an event-by-event basis.

\subsection{Color Structure of Heavy Quark Production Processes}

The study of heavy flavor production is one of the main objectives of the collider experiments of the present and of the future. Apart from the intrinsic QCD aspects of jets generated by heavy quarks which were under focus in 
Chapter [4 this subject is of large importance for studies of the fundamental properties of heavy particles, such as spatial oscillations of flavor and $\mathrm{CP}$ violating effects in their decays. Recall also that heavy flavors dominate in the quark decay modes of new objects such as the Higgs boson.

Here we extend the analysis of soft QCD radiation to processes involving heavy quarks. Antenna patterns are briefly discussed for some particularly interesting reactions. Details on this subject one can find in Refs. 910.

First of all, let us point out some specific properties of soft emission from a colorless pair of massive quarks $Q \bar{Q}^{\prime}(Q \bar{Q}$-antenna) 10/11]. In this case the radiation pattern is determined by the classical current (notation are the same as in Fig. (4.9) and may be written as (cf. (4.12), (4.13))

$$
\begin{aligned}
& d w_{Q \bar{Q}^{\prime}}=\frac{d k}{k} d \Omega_{\vec{n}} \frac{\alpha_{s}}{(2 \pi)^{2}} W^{Q \bar{Q}^{\prime}}, \\
& W^{Q \bar{Q}^{\prime}}=2 C_{F} \widehat{(i j)}_{m}=2 C_{F}\left(\frac{\tilde{a}_{i j}}{\tilde{a}_{i} \tilde{a}_{j}}-\frac{1}{2 \gamma_{i}^{2} \tilde{a}_{i}^{2}}-\frac{1}{2 \gamma_{i}^{2} \tilde{a}_{j}^{2}}\right),
\end{aligned}
$$

with $\beta_{i}$ denoting the velocity of quark $i$ in the relations

$$
\tilde{a}_{i j}=1-\beta_{i} \beta_{j} \cos \Theta_{i j}, \quad \tilde{a}_{i}=1-\beta_{i} \cos \Theta_{i}, \quad \gamma_{i}=\frac{E_{i}}{m_{i}}=\frac{1}{\sqrt{1-\beta_{i}^{2}}} .
$$

From (10.10) one can see that the collinear singularity is removed from the physical region by the quark mass $m_{i}$. The effect of the second (third) term in (10.10) is to suppress the radiation in the region $\tilde{a}_{i} \leq \gamma_{i}^{-2} \quad\left(\tilde{a}_{j} \leq \gamma_{j}^{-2}\right)$, introducing an effective cutoff $\cos \Theta_{i} \leq \beta_{i} \quad\left(\cos \Theta_{j} \leq \beta_{j}\right)$. Similarly to the massless quark case, interference between the radiation from $i$ and $j$ tends to suppress values of $\tilde{a}_{i}$ and $\tilde{a}_{j}$ above $\tilde{a}_{i j}$.

After azimuthal averaging the emission of a soft gluon by a heavy quark of velocity $\beta_{i}$ effectively takes place only in the following "screened cone" 10 
(cf. (4.16)):

$$
\beta_{i} \cos \Theta_{i j}<\cos \Theta_{i}<\cos \Theta_{i}^{\min } \approx \beta_{i},
$$

with $\beta_{j}$ the velocity of the quark which is color connected to the emitting one. Equation (10.12) is convenient for treatment of heavy quark jet fragmentation in Monte Carlo simulations.

Production of heavy quarks $Q$ in hadronic collisions is governed by the light-quark and gluon fusion mechanisms

$$
\begin{aligned}
& q\left(p_{1}\right)+\bar{q}\left(p_{2}\right) \rightarrow Q\left(p_{3}\right)+\bar{Q}\left(p_{4}\right), \\
& g\left(p_{1}\right)+g\left(p_{2}\right) \rightarrow Q\left(p_{3}\right)+\bar{Q}\left(p_{4}\right) .
\end{aligned}
$$

Of special interest are electroweak subprocesses such as Higgs boson and $Z^{0}$ production

$$
\begin{aligned}
& g\left(p_{1}\right)+g\left(p_{2}\right) \rightarrow H^{0} \rightarrow Q\left(p_{3}\right)+\bar{Q}\left(p_{4}\right), \\
& q\left(p_{1}\right)+\bar{q}\left(p_{2}\right) \rightarrow Z^{0} \rightarrow Q\left(p_{3}\right)+\bar{Q}\left(p_{4}\right) .
\end{aligned}
$$

The light-quark fusion process $(i)$ has a unique color structure, shown in Fig. 10.11(a). The invariant matrix element squared $\sigma_{s}^{Q}$ is given by (cf. with $\sigma_{s}$ in (A.6) for the massless case):

$$
\begin{gathered}
\sigma_{s}^{Q}=\frac{C_{F}}{N_{c}}\left(\frac{t^{\prime 2}+u^{\prime 2}}{s^{\prime 2}}+\frac{2 m_{Q}^{2}}{s^{\prime}}\right), \\
s^{\prime}=\left(p_{1}+p_{2}\right)^{2}=2 p_{1} p_{2}, \\
\text { with } \quad \begin{aligned}
t^{\prime} & =\left(p_{1}-p_{3}\right)^{2}-m_{Q}^{2}=-2 p_{1} p_{3}, \\
u^{\prime} & =\left(p_{1}-p_{4}\right)^{2}-m_{Q}^{2}=-2 p_{1} p_{4} .
\end{aligned}
\end{gathered}
$$



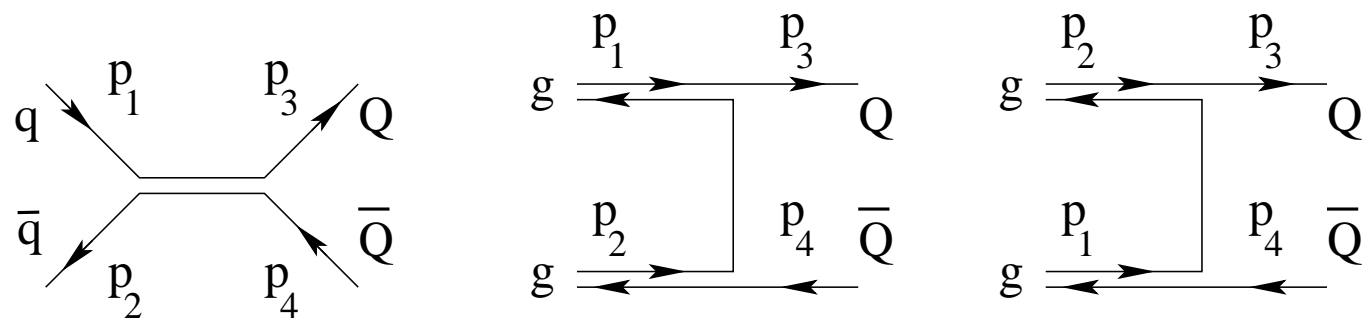

Figure 10.11: Color antenna configurations for heavy quark production subprocesses: (d) $q \bar{q} \rightarrow Z^{0} \rightarrow Q \bar{Q}$.

(a) $q \bar{q} \rightarrow Q \bar{Q}$,

(b) $g g \rightarrow Q \bar{Q}$,

(c) $g g \rightarrow H \rightarrow Q \bar{Q}$,

The amplitude of the gluon fusion (ii) has two possible color structures as shown in Fig. 10.11(b). The invariant matrix element squared $\sigma_{G}$ can be written as a sum of two terms (see Refs. [101213]):

$$
\begin{gathered}
\sigma_{G}=G\left(s^{\prime}, t^{\prime}, u^{\prime}\right)+G\left(s^{\prime}, u^{\prime}, t^{\prime}\right) \\
G\left(s^{\prime}, t^{\prime}, u^{\prime}\right)=\frac{N_{c}}{N_{c}^{2}-1}\left[\frac{u^{\prime}}{2 t^{\prime}}-\frac{u^{\prime 2}}{s^{\prime 2}}-\frac{2 m_{Q}^{2}}{t^{\prime}}-\frac{2 m_{Q}^{2}}{s^{\prime}}-\frac{2 m_{Q}^{4}}{t^{\prime 2}}\right. \\
\left.-\frac{1}{N_{c}^{2}}\left(\frac{u^{\prime}}{2 t^{\prime}}-\frac{2 m_{Q}^{2}}{t^{\prime}}-\frac{2 m_{Q}^{4}}{t^{\prime 2}}+\frac{4 m_{Q}^{4}}{s^{\prime} t^{\prime}}\right)\right] .
\end{gathered}
$$

The two terms in $\sigma_{G}$ have clear correspondence to the two diagrams of Fig. 10.11(b). The planar parts of $G$ are just the squared amplitudes for the different color flow structures. The suppressed by $1 / N_{c}^{2}$, the non-planar contribution to $G$, corresponds to the interference of these amplitudes. 
For the subprocesses (iii) and (iv), the color structure is very simple: the initial and final states separately form color singlets, as shown in Fig. 10.11(c),(d). The radiation pattern, corresponding to unique color structure of Fig. 10.11(a) can be approximated as

$$
\frac{4 \pi d N_{(i)}}{d \Omega_{\vec{n}}}=\left[{\widehat{(13)_{m}}}+\widehat{(24)}_{m}\right] \frac{C_{F}}{N_{c}} N_{g}^{\prime}
$$

where $(\widehat{(i j)})_{m}$ is given by (10.10), (10.11). The terms neglected in (10.18) is doubly suppressed: first by $1 / N_{c}^{2}$, and second by the fact that it is not collinear singular.

The antenna pattern for the gluon fusion subprocess (ii) can be written to leading order in $1 / N_{c}$ as

$$
\frac{4 \pi d N_{(i i)}}{d \Omega_{\vec{n}}}=\frac{1}{2}\left[\left(\widehat{(13)}_{m}+\widehat{(24)}_{m}+\widehat{(12)}\right) \delta_{t^{\prime}}+\left(\widehat{(14)}_{m}+\widehat{(23)}_{m}+\widehat{(12)}\right) \delta_{u^{\prime}}\right] N_{g}^{\prime},
$$

with $\delta_{t^{\prime}}=G\left(s^{\prime}, t^{\prime}, u^{\prime}\right) / \sigma_{G}, \delta_{u^{\prime}}=G\left(s^{\prime}, u^{\prime}, t^{\prime}\right) / \sigma_{G}$ defined by (10.16), (10.17).

Finally, in the Higgs boson and $Z^{0}$ production processes the antenna patterns are given by

$$
\begin{aligned}
& \frac{4 \pi d N_{(i i i)}}{d \Omega_{\vec{n}}}=\left[\frac{N_{c}}{C_{F}} \widehat{(12)}+\widehat{(34)_{m}}\right] \frac{C_{F}}{N_{c}} \cdot N_{g}^{\prime}, \\
& \frac{4 \pi d N_{(i v)}}{d \Omega_{\vec{n}}}=\left[\widehat{(12)}+\widehat{(34)_{m}}\right] \frac{C_{F}}{N_{c}} \cdot N_{g}^{\prime} .
\end{aligned}
$$

\subsection{Azimuthal Asymmetry of QCD Jets}

As we have already discussed in Section 9.4 the treatment of the structure of final states given by the string picture qualitatively reproduces the QCD 
radiation pattern only up to $O\left(1 / N_{c}^{2}\right)$ corrections (the large- $N_{c}$-limit). Such corrections are mainly neglected in all the parton shower algorithms. The reason is that these terms often appear with a negative sigh, or correspond to events with an undefined color flow, and so it is not clear how to handle them in Monte Carlo shower simulations (see Refs. 114). Normally the neglect is not drastic, but under special conditions $1 / N_{c}^{2}$ terms may become sizable or even dominant.

\subsubsection{Jet Asymmetry in $e^{+} e^{-}$annihilation}

The simplest example is given by the azimuthal asymmetry of a quark jet in $q \bar{q} g$ events. The radiation pattern is given here by (9.34) When all the angles are large the third term in (9.34) (negative color-suppressed antenna $(+-))$ leads to a small correction to the canonical string interpretation of the drag effect. However if the $q$ and $\bar{q}$ are close to each other, this term is kinematically enhanced, and is no longer negligible.

The azimuthal distribution of particles produced inside a cone of given opening half-angle $\Theta_{0}$ may be characterized by an asymmetry parameter (see Fig. 10.12)

$$
A\left(\Theta_{0}\right)=\frac{N_{\rightarrow g}\left(\Theta<\Theta_{0}\right)-N_{\rightarrow \bar{q}}\left(\Theta<\Theta_{0}\right)}{N_{t o t}\left(\Theta<\Theta_{0}\right)}=\frac{(\Delta N)_{a s}}{N_{t o t}} .
$$

The azimuthal integration for $(\Delta N)_{a s}$,

$$
(\Delta N)_{a s}=\left[\int_{-\pi / 2}^{\pi / 2}-\int_{\pi / 2}^{3 \pi / 2}\right] \frac{d \phi}{2 \pi} \cdot\left(\frac{8 \pi \Delta N}{d \Omega}\right),
$$



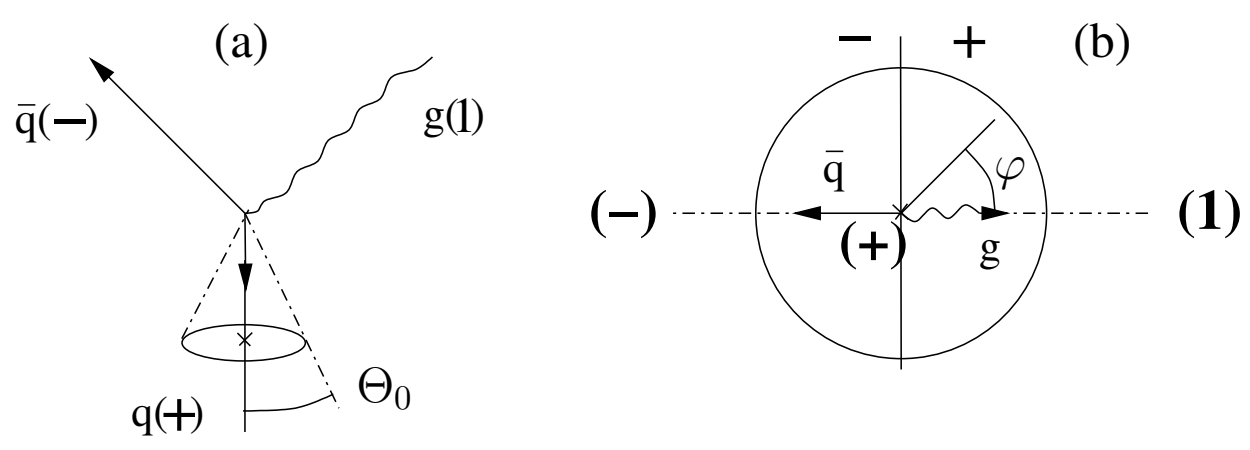

Figure 10.12: The azimuthal asymmetry of the quark jet in $e^{+} e^{-} \rightarrow q \bar{q} g$. (a) Geometry of the event plane, dashed lines show the topology of color strings. (b) Scheme of azimuthal separation of particles from quark jet, see (10.21), (10.22).

can be done explicitly. For parametrically small $\Theta_{0}$ values the contribution of the $\widehat{(+i)}$-antenna $(i=-, 1)$ to $(\Delta N)_{a s}$ reads

$$
\frac{1}{2} \int_{0}^{\Theta_{0}} \frac{d \Theta_{+}}{\Theta_{+}}\left(\frac{2 \Theta_{+}}{\pi} \cot \frac{\Theta_{+i}}{2}\right) N_{g}^{\prime}\left(\ln E \Theta_{+}\right) \approx \frac{\Theta_{0}}{\pi} \cot \frac{\Theta_{+i}}{2} N_{g}^{\prime}\left(\ln E \Theta_{0}\right),
$$

while the nonsingular antenna $\widehat{(-1)}$ produces negligible correction $(\Delta N)_{a s} \sim$ $\Theta_{0}^{3}$. The resulting asymmetry parameter takes the form

$$
\begin{aligned}
A\left(\Theta_{0}\right) & \approx \frac{2 \Theta_{0}}{\pi} G \sqrt{4 N_{c} \frac{\alpha_{s}\left(E \Theta_{0}\right)}{2 \pi}}, \\
G & =\frac{N_{c}}{2 C_{F}} \cot \frac{\Theta_{+1}}{2}+\frac{1}{2 N_{c} C_{F}} \cot \frac{\Theta_{+-}}{2},
\end{aligned}
$$


where we have substituted

$$
\frac{N_{g}^{\prime}\left(\ln E \Theta_{0}\right)}{N_{g}\left(\ln E \Theta_{0}\right)}=\sqrt{4 N_{c} \frac{\alpha_{s}\left(E \Theta_{0}\right)}{2 \pi}} \cdot\left(1+O\left(\sqrt{\frac{\alpha_{s}}{\pi}}\right)\right) .
$$

The first color-favored term in (10.24) describes the string model motivated asymmetry due to the "boosted string segment" connecting $q$ - and $g$-directions. The corresponding asymmetry vanishes with increase of $\Theta_{+1}$ as the string piece straightens.

Here, however, the second term of (10.24) enters the game, forcing the asymmetry to increase anew as shown in Fig. 10.13(a). This behavior might be interpreted as an additional repulsion between particles from two neighboring $q$ - and $\bar{q}$-jets. Let us remind the reader that in the Lund string scenario no direct color connection exists between the quarks.

For symmetric configuration,

$$
\Theta_{+1}=\Theta_{-1}=\pi-\Theta_{+-} / 2
$$

the color-suppressed term prevails when

$$
\Theta_{+-} \leq 2 \arctan \frac{1}{N_{c}} \approx 37^{0}
$$

To realize this effect one has to select $q \bar{q} g$ events with rear kinematics, when a hard gluon moves in opposite direction to the quasi-collinear $q \bar{q}$ pair. Fig. 10.13(b) displays the predicted asymptotical asymmetry of the quark jet at finite values of $\Theta_{0}$ as a function of the relative angle between the $q$ - and $\bar{q}$-jets. The increase of $A$ with decreasing $\Theta_{q \bar{q}}$ can be seen only for very small values of $\Theta_{0}$. The reason is that for $\Theta_{0} \gtrsim 5^{0}$ the effect of repulsion is masked by the fragments of the neighboring $\bar{q}$-jet which partially fill the $\Theta_{0}$-cone, leading to a drastically increasing negative contribution to $A$. 
a)

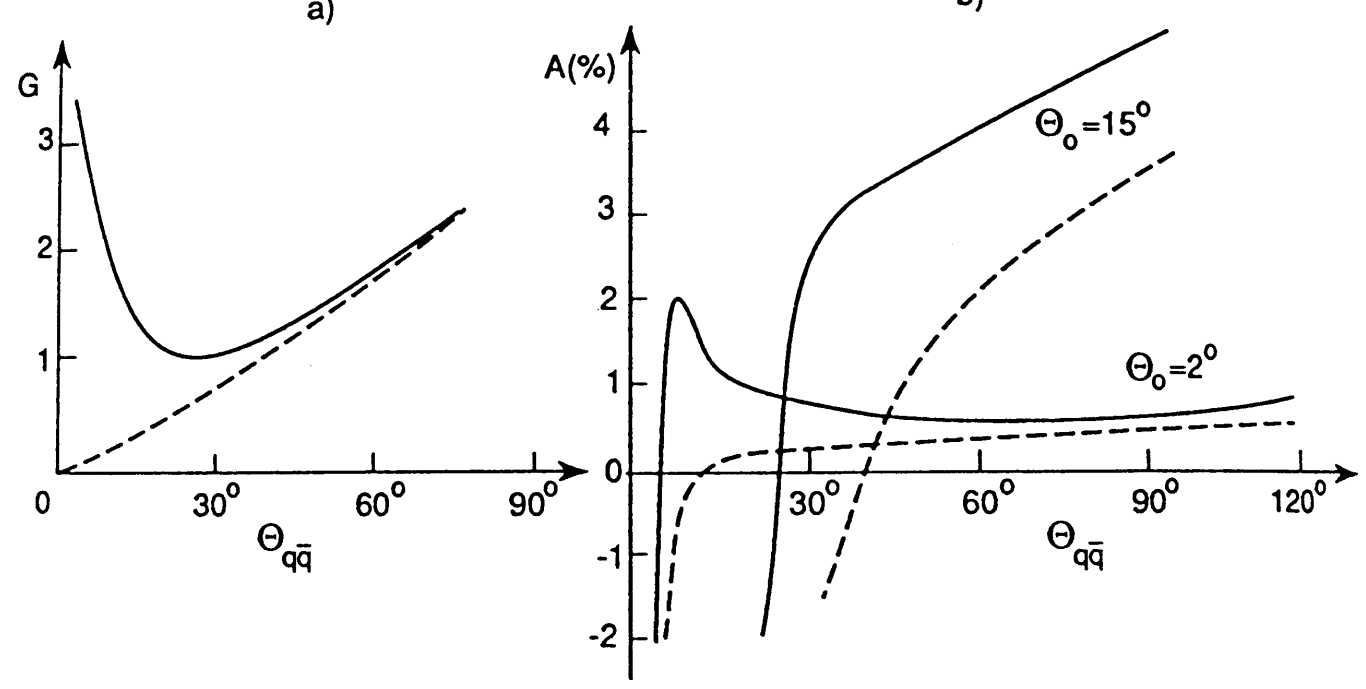

Figure 10.13: QCD (solid) versus large- $N_{c}$-limit (dashed) predictions for (a) $G$-factor of (10.23) and

(b) asymmetry parameter $A$ in the symmetric $q \bar{q} g$ events.

The abovementioned phenomenon can be studied experimentally by looking at the azimuthal distribution of particles around the identified quark direction, which can be most conveniently done by tagging heavy flavor quark jets. An interesting vista on this problem is connected with the $Z^{0} \rightarrow c \bar{c} g$ events. As one can see from Fig. 10.13(b), the increase of $A$ is of comfortable size at moderate angles $\Theta_{c \bar{c}}$. 


\subsubsection{Jet Asymmetry in hadron scattering}

The azimuthal asymmetry of produced jets is certainly not specific to 3-jet events. An analogous picture should be observed, e.g., in high- $p_{\perp}$ processes (see Ref. 11 for details). The qualitative difference between the predictions of QCD and its large- $N_{c}$-limit proves to be the $p \bar{p}$ scattering with the identification of the scattered $q$-jet.

To elucidate the asymmetry phenomenon in high- $p_{\perp}$ processes let us compare the angular pattern of the radiation accompanying $q q^{\prime}$ and $\bar{q} q^{\prime}$ scattering (where $q$ and $q^{\prime}$ have different flavors). The corresponding particle distributions are given by (10.3) and (A.1), (A.2). Similar to the $q \bar{q} g$ example, the first color-favored terms in these equations correspond topologically to canonical string pieces. "Boosted" $\widehat{(14)}$ and $\widehat{(23)}$ antennae for the quark scattering lead to the azimuthal asymmetry of the jets. Unlike this case, drawing the string picture for $\bar{q} q^{\prime}$ scattering one should expect no such asymmetry since both $\widehat{(12)}$ and $\widehat{(34)}$ antennae appear to be straight (each string piece is in its cms).

In the case of $q q^{\prime}$ scattering the color-suppressed term leads to some deviation from the Lund motivated asymmetry. Meantime, for the $\bar{q} q^{\prime}$ scattering such a term determines the whole effect.

For illustration let us consider the azimuthal distribution of particles inside the jet \#3. Determining the asymmetry parameter $A\left(\Theta_{0}\right)$ analogously to (10.21) (see Fig. 10.14),

$$
A\left(\Theta_{0}\right)=\frac{N_{\rightarrow 2}\left(\Theta<\Theta_{0}\right)-N_{\rightarrow 1}\left(\Theta<\Theta_{0}\right)}{N_{t o t}\left(\Theta<\Theta_{0}\right)},
$$




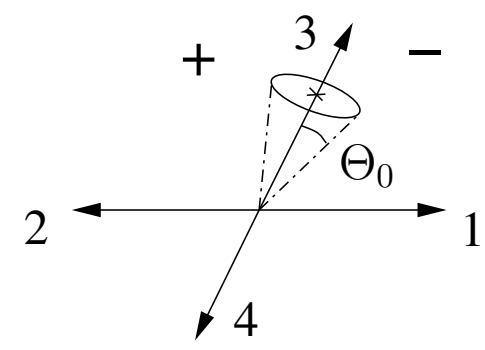

(a)

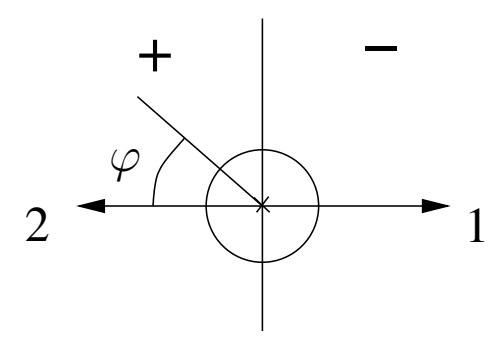

(b)

Figure 10.14: Definition of the azimuthal asymmetry $A\left(\Theta_{0}\right)$ of the scattered jet \#3.

one obtains for small opening half-angle $\Theta_{0}$ around the jet-\#3 direction

$$
\begin{gathered}
A^{q q^{\prime}}\left(\Theta_{0}\right)=\widetilde{\gamma_{0}} \frac{2 \Theta_{0}}{\pi}\left[\frac{N_{c}}{2 C_{F}} \tan \frac{\Theta_{s}}{2}+\frac{1}{2 N_{c} C_{F}}\left(\cot \frac{\Theta_{s}}{2}-2 \tan \frac{\Theta_{s}}{2}\right)\right], \\
A^{\bar{q} q^{\prime}}\left(\Theta_{0}\right)=\widetilde{\gamma_{0}} \frac{2 \Theta_{0}}{\pi}\left[\frac{1}{2 N_{c} C_{F}}\left(2 \tan \frac{\Theta_{s}}{2}+\cot \frac{\Theta_{s}}{2}\right)\right] .
\end{gathered}
$$

Here

$$
\widetilde{\gamma}_{0}=\sqrt{4 N_{c} \frac{\alpha_{s}\left(E \Theta_{0}\right)}{2 \pi}}
$$

and $\Theta_{s}$ is the scattering angle:

$$
\Theta_{13}=\Theta_{24}=\Theta_{s}, \quad \Theta_{23}=\Theta_{14}=\pi-\Theta_{s} .
$$

Fig. 10.15 demonstrates the comparison of event shape factor $G$ (factors in square brackets in (10.25)) with their large- $N_{c}$-limits. The $O\left(1 / N_{c}^{2}\right)$ term in 


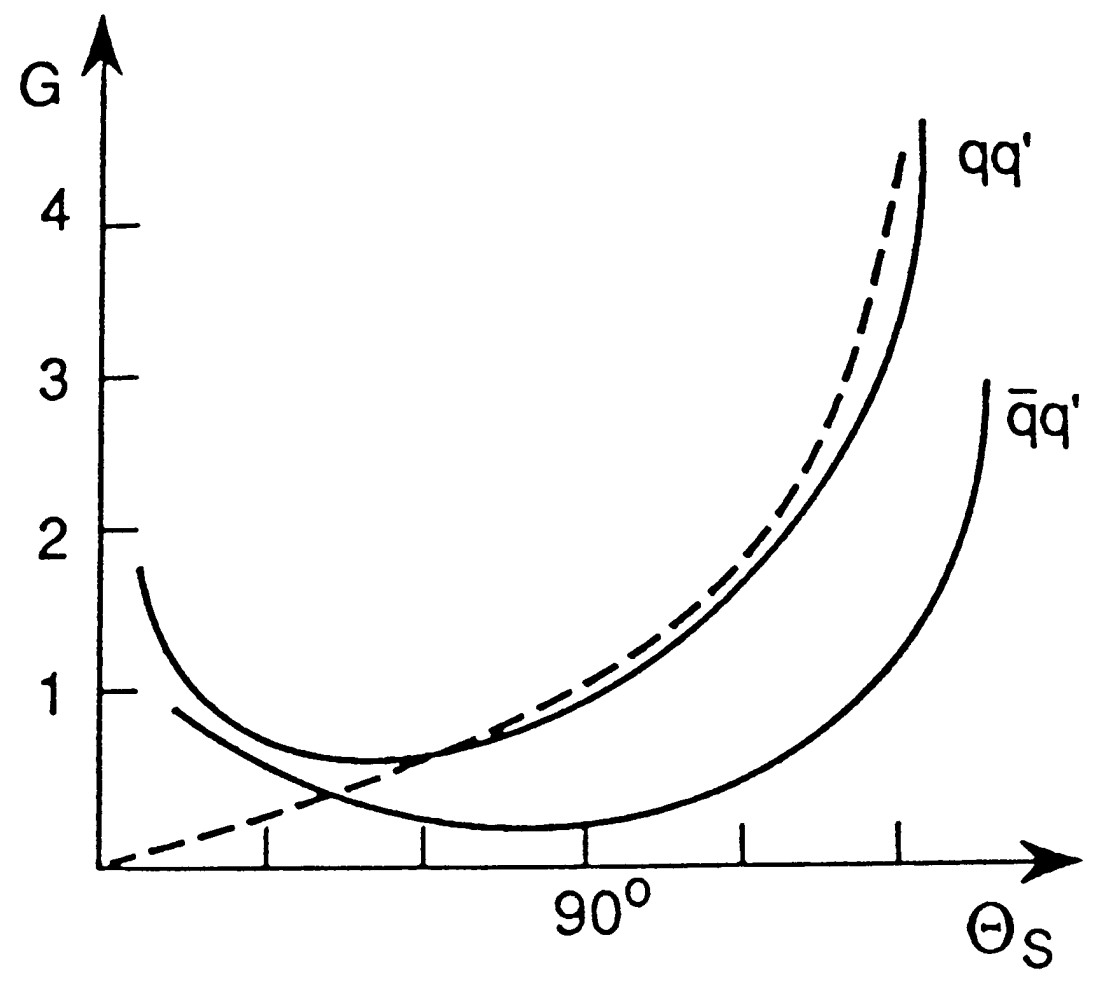

Figure 10.15: $G$-factor for $q q^{\prime}$ and $\bar{q} q^{\prime}$ scattering. Solid - QCD, dashed large- $N_{c}$ limit for $q q^{\prime}$ case (for $\bar{q} q^{\prime}$ scattering string scenario predicts $G=0$ ). 
(10.25a) dominates for scattering angles

$$
\Theta_{s}<2 \arctan \sqrt{\frac{1}{N_{c}^{2}+2}} \approx 33.6^{0} .
$$

The predicted magnitude of the QCD asymmetry in $\bar{q} q^{\prime}$ scattering appears to be comparable with that of the $q q^{\prime}$ case. For example,

$$
\begin{array}{ll}
A^{\bar{q} q^{\prime}} \approx A^{q q^{\prime}} & \text { at small } \Theta_{s}, \\
A^{\bar{q} q^{\prime}} \approx \frac{3}{8} A^{q q^{\prime}} & \text { at } \Theta_{s}=90^{\circ} .
\end{array}
$$

Notice, that to study the azimuthal properties of jet \#3 the natural restriction $\Theta_{s}>2 \Theta_{0}$ has to be imposed.

Recall that in the case of identical quarks new effects arise leading to the complication of the antenna patterns via

- modification of the $q q$ scattering amplitude and

- opening of the annihilation channel for the $\bar{q} q$ case.

Keeping in mind that the asymmetry of the jet $\# 3$ comes mainly from $\widehat{(13)}$ and $\widehat{(23)}$ antennae, one can simply observe that in the case of $\bar{q} q$ scattering this asymmetry, unlike the case of distinguishable quarks, contains also the color-favored term $\widehat{(13)} \cdot R_{s}$, caused by the annihilation contribution. This term produces a negative asymmetry, opposite to the positive one due to the remaining terms. Note, the color-favored negative asymmetry occurs also for the $q \bar{q} \rightarrow q^{\prime} \bar{q}^{\prime}$ and $q \bar{q} \rightarrow 2 g$ subprocesses. We emphasize that just the color-suppressed $O\left(1 / N_{c}^{2}\right)$ term proves to govern the overall asymmetry here owing to the numerical smallness of the annihilation cross section $\left(\sigma_{s} / \sigma_{q \bar{q}} \lesssim\right.$ 
1/10 for $\Theta_{s}<90^{\circ}$, see Appendix). One concludes, thus, that QCD differs qualitatively from the canonical string picture in predicting the asymmetry of the azimuthal jet profile. In this point we face for the first time the situation when QCD and its large- $N_{c}$-limit gave opposite sign predictions.

Quark-antiquark scattering can be studied in $p \bar{p}$ collisions at high- $p_{\perp}$. Here both $q \bar{q}$ and $q \bar{q}^{\prime}$ scattering occur. To make the qualitative difference

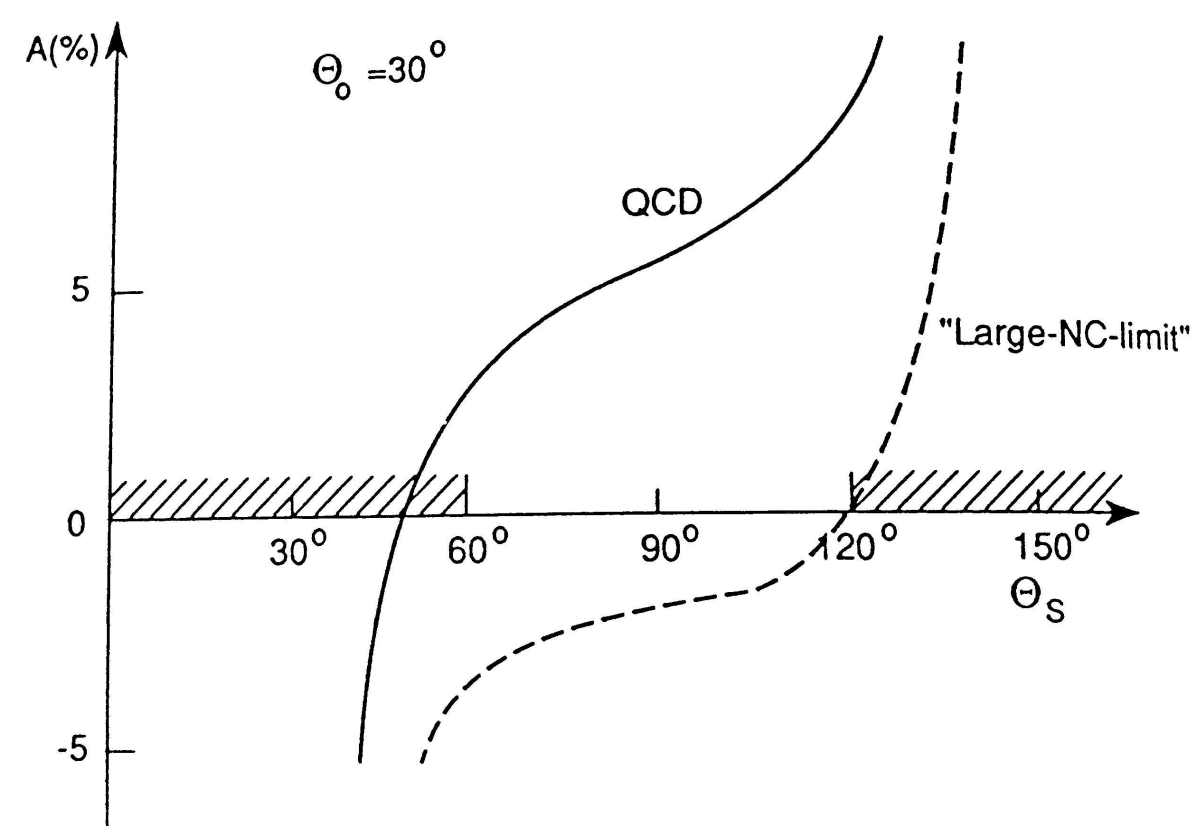

Figure 10.16: Asymmetry parameter $A\left(\Theta_{0}=30^{\circ}\right)$ of the scattered tagged $q$-jet in $p \bar{p}$-collisions 1 . 
between the predictions of QCD and its large- $N_{c}$-limit most spectacular it is necessary to identify the scattered quark jet. The asymmetry predicted for the case of a tagged quark jet is shown in Fig. 10.16. As we see, in the region of $\mathrm{cms}$ scattering angles $70^{\circ}<\Theta_{s}<110^{\circ}$ QCD predicts jet asymmetry

$$
A=+(4 \div 7) \% \quad \text { at the half-angle } \Theta_{0}=30^{\circ}
$$

unlike the opposite sign effect

$$
A=-(2.5 \div 1.5) \%
$$

originated from the large- $N_{c}$ treatment of QCD formulae, equivalent to the string-motivated approach.

\subsection{Lessons}

Concluding this Chapter let us enumerate the main lessons from studying the collective QCD effects — interjet coherence phenomena.

1. The effects of gluon interference do not permit one to formulate, $a$ priori, a probabilistic scheme for the development of partonic system. However for each specific inclusive characteristic it is possible to divide the essential coherent effects into two types:

(a) accounts for the coherence effects in the intrajet cascades. These are reduced on average to the Markov process of independent particle multiplication into sequentially shrinking angular cones $\Theta_{n+1} \leq \Theta_{n}$

(b) gives an account of the interference effects in the total amplitude for production of the minimal number of jets (partons), whose 
configuration corresponds to the given experiment. Just this amplitude reflects specific features of a hard collision. Each of the produced jets evolves in an universal manner inside a fixed opening angle $\Theta_{0}$, which size depends on the mutual location of jets from the ensemble.

2. The aforementioned division permits, in each concrete case, a classical probabilistic picture of parton branching to be retained, thus allowing the use of Monte Carlo simulations. However the collective phenomena in the multijet ensembles could be reproduced with use of classical probabilistic language only approximately, in large $N_{c}$ limit. The problem is that nowadays one faces an unresolved problem how to handle non-positive definite $1 / N_{c}^{2}$ terms in Monte Carlo algorithms.

3. The experimental evidence of such bright phenomena as the humpbacked plateau (coherence of the first kind) and the drag effect (coherence of the second kind) has shown quite convincingly that these interference effects survive the hadronization stage. Therefore, one can say, that in spite of confinement the hadronic system reflects very delicate features of the color field dynamics, which in turn stem from the nature of QCD as a gauge theory.

4. The observation of the color interference between soft hadrons from, say, $q$ - and $g$-jets reveals the QCD wave properties of hadronic flows. Thus, it can be considered as an experimental proof of the common bremsstrahlung nature of the hadroproduction mechanisms for both jets. The properties of drag phenomena are deeply rooted in the basic structure of nonabelian gauge theory. 
5. Drag effects lead to a noticeable azimuthal asymmetry of particle flow relative to the "jet axis". The character of this asymmetry depends on the geometry of the whole event.

6. The relative smallness of some nonclassical effects, e.g., in azimuthal jet asymmetry or in color screening does not diminish their fundamental importance. This consequence of QCD radiophysics is a serious warning against the ideas about independently evolving jets. 
Problem 10.1 Derive the expression for the average of the heavy quark antenna $\widehat{(i j)}_{m}$ over the azimuthal angle, see (10.10).

\section{Solution:}

Analogously to the massless case (see Section 4.4) let us represent $\widehat{(i j)}_{m}$ in the form

$$
\widehat{(i j)}_{m}=\left(P_{i j}\right)_{m}+\left(P_{j i}\right)_{m}
$$

where

$$
\begin{aligned}
\left(P_{i j}\right)_{m} & =\frac{\beta_{i}}{2 \tilde{a}_{i}}\left(\frac{A_{i}}{1-\beta_{i} \cos \Theta_{i}}+\frac{B_{i}}{1-\beta_{j} \cos \Theta_{j}}\right) \\
A_{i} & =\beta_{i}-\cos \Theta_{i}, \quad B_{i}=\cos \Theta_{i}-\beta_{j} \cos \Theta_{i j}
\end{aligned}
$$

and similarly for $\left(P_{j i}\right)_{m}$, which could be associated with radiation from partons $i$ and $j$ respectively. Using

$$
\left\langle\frac{1}{1-\beta_{j} \cos \Theta_{j}}\right\rangle=\frac{1}{\sqrt{B_{i}^{2}+\left(\sin \Theta_{i} / \gamma_{j}\right)^{2}}}
$$

one gets

$$
\left\langle\left(P_{i j}\right)_{m}\right\rangle=\frac{\beta_{i}}{2 \tilde{a}_{i}}\left(\frac{A_{i}}{\beta_{i} A_{i}+\gamma_{i}^{-2}}+\frac{B_{i}}{\sqrt{B_{i}^{2}+\left(\sin \Theta_{i} / \gamma_{j}\right)^{2}}}\right) .
$$

From (10.26), (10.27) one can derive the relation (10.12), see Ref. 10.

Problem 10.2 Calculate the contribution to $\Delta N_{\text {as }}$ (see (10.22)) from antennae $\widehat{(+i)}(i=-, 1)$ and $\widehat{(-1)}$. Analyze the behavior at small values of $\Theta_{+}$. 


\section{Answer:}

$$
\Delta N_{+i}=s_{i} \frac{a_{+i}}{a_{+}\left|a_{+}-a_{+i}\right|} \cdot \frac{2}{\pi} \arctan \frac{\sin \Theta_{+} \sin \Theta_{+i}}{\left|\cos \Theta_{+}-\cos \Theta_{+i}\right|}
$$

$s_{-}=-1, \quad s_{1}=1 ; \quad a_{i j}=1-\vec{n}_{i} \vec{n}_{j}=1-\cos \Theta_{i j}, \quad a_{i}=1-\vec{n} \vec{n}_{i}=1-\cos \Theta_{i}$, see Fig. 10.12

$$
\begin{aligned}
\Delta N_{-1}= & \frac{1-\cos \Theta_{-1}}{\sin \Theta_{+-}+\sin \Theta_{1+}+\sin \Theta_{-1} \cos \Theta_{+}} \sum_{i=-, 1} s_{i} \frac{\sin \Theta_{+i}}{\left|\cos \Theta_{+}-\cos \Theta_{+i}\right|} \\
& \cdot \frac{2}{\pi} \arctan \frac{\sin \Theta_{+} \sin \Theta_{+i}}{\left|\cos \Theta_{+}-\cos \Theta_{+i}\right|} .
\end{aligned}
$$

With $\Theta_{+} \rightarrow 0$

$$
\begin{aligned}
\Delta N_{+i} & \approx s_{i} \frac{\sin \Theta_{+}}{1-\cos \Theta_{+}} \cdot \frac{2}{\pi} \cot \frac{\Theta_{+i}}{2} \propto \frac{1}{\Theta_{+}} \\
\Delta N_{-1} & \propto \Theta_{+} .
\end{aligned}
$$

Problem 10.3 Prove that in the case of $p \bar{p}$ collisions $Q C D$ and its large- $N_{c^{-}}$ limit lead to the opposite sign azimuthal asymmetry of quark jet at large $\mathrm{cms}$ scattering angles. 


\section{Appendix}

\section{Radiation Patterns for Parton-Parton Scattering}

The aim of this is to collect the formulae describing the radiation patterns for some $2 \rightarrow 2$ hard processes. The reader can find in Refs. [16] more details. The antenna pattern for the hard $q(\bar{q})$ scattering may be written as

$$
\frac{4 \pi d N}{d \Omega_{\vec{n}}}=\left[\widehat{(12)}+\widehat{(34)}+\hat{D}_{t s} R_{u}+\hat{D}_{u s} R_{t}\right] \frac{C_{F}}{N_{c}} \cdot N_{g}^{\prime}
$$

Here

$$
\begin{aligned}
& \hat{D}_{t s}=\widehat{(13)}+\widehat{(24)}-\widehat{(12)}-\widehat{(34)}=-\hat{D}_{s t} \\
& \hat{D}_{u s}=\widehat{(14)}+\widehat{(23)}-\widehat{(12)}-\widehat{(34)}=-\hat{D}_{s u} \\
& \hat{D}_{t u}=-\hat{D}_{u t}=\hat{D}_{t s}-\hat{D}_{u s}
\end{aligned}
$$

are the "dipole" combinations of different antennae with subscripts $s, t, u$ defined according to (10.5). Dipoles $\hat{D}$ prove to be less singular than the leading term $(\widehat{(12)}+\widehat{(34)})$. They are responsible, e.g., for the azimuthal asymmetry of jets studied in Section 10.6.

The functions $R_{u}(s, t, u), R_{t}(s, t, u)$ are uniquely determined for each elementary $2 \rightarrow 2$ hard subprocesses.

For the case of scattering of quarks with different flavors

$$
q_{1} q_{2}^{\prime} \rightarrow q_{3} q_{4}^{\prime}
$$


one has

$$
R_{t}=1+R_{u}, \quad R_{u}=-\frac{1}{2 N_{c} C_{F}} .
$$

For the same flavor quarks

$$
q_{1} q_{2} \rightarrow q_{3} q_{4}
$$

the exchange amplitude enters the game and these functions become more complicated:

$$
\begin{aligned}
& R_{t}=\frac{\sigma_{t}}{\sigma_{q q}}-\frac{1}{2 N_{c} C_{F}} \quad, \quad R_{u}=\frac{\sigma_{u}}{\sigma_{q q}}-\frac{1}{2 N_{c} C_{F}}, \\
& \sigma_{t}=\frac{C_{F}}{N_{c}} \frac{s^{2}+u^{2}}{t^{2}} \quad, \quad \sigma_{u}=\frac{C_{F}}{N_{c}} \frac{s^{2}+u^{2}}{u^{2}} ; \\
& \sigma_{q q}=\sigma_{t}+\sigma_{u}-\Delta_{t u} \quad, \quad \Delta_{t u}=\frac{C_{F}}{N_{c}^{2}} \frac{2 s^{2}}{t u} .
\end{aligned}
$$

Here $\sigma_{t}\left(\sigma_{u}\right)$ is the contribution to the $q q$-scattering cross section coming from the $t(u)$-channel gluon exchange, with $\Delta_{t u}$ the interference term.

For scattering of quark and antiquark of different flavors

$$
\bar{q}_{1} q_{2}^{\prime} \rightarrow \bar{q}_{3} q_{4}^{\prime}
$$

one has

$$
R_{t}=-2 R_{u}, \quad R_{u}=-\frac{1}{2 N_{c} C_{F}} .
$$

When $q$ and $\bar{q}$ are of the same flavor

$$
\begin{array}{r}
R_{t}=-\frac{\Delta_{t s}}{\sigma_{q \bar{q}}}+\frac{1}{N_{c} C_{F}} \quad, \quad R_{u}=\frac{\sigma_{s}}{\sigma_{q \bar{q}}}-\frac{1}{2 N_{c} C_{F}}, \\
\sigma_{s}=\frac{C_{F}}{N_{c}} \frac{t^{2}+u^{2}}{s^{2}}, \\
\sigma_{q \bar{q}}=\sigma_{t}+\sigma_{s}-\Delta_{t s} \quad, \quad \Delta_{t s}=\frac{C_{F}}{N_{c}^{2}} \frac{2 u^{2}}{t s} .
\end{array}
$$


Here $\sigma_{s}$ is the contribution of the annihilation channel, $\sigma_{t}$ is given by (A.4), and $\Delta_{t s}$ is the interference term. Notice the color suppression of interference contributions $\Delta_{t s}=\Delta_{t u}(u \leftrightarrow s)$.

The radiation pattern for the annihilation channel

$$
q_{1}+\bar{q}_{2} \rightarrow g_{3}+g_{4}
$$

slightly differs from that of (A.1):

$$
\begin{gathered}
\frac{4 \pi d N}{d \Omega_{\vec{n}}}=\left[\widehat{(12)}+\frac{N_{c}}{C_{F}} \widehat{(34)}+\hat{D}_{t s} R_{u}+\hat{D}_{u s} R_{t}\right] \frac{C_{F}}{N_{c}} \cdot N_{g}^{\prime} ; \\
R_{u}=\frac{u^{2} / s^{2}-1 / N_{c}^{2}}{2\left(\left(t^{2}+u^{2}\right) / s^{2}-1 / N_{c}^{2}\right)}, \quad R_{t}=R_{u}(t \leftrightarrow u) .
\end{gathered}
$$

We list below also formulae for the three other subprocesses assuming the dominance of $t$-channel exchange (small scattering angle $\Theta_{13} \ll 1$ ).

- $g_{1}+q_{2} \rightarrow g_{3}+q_{4}$

$$
\frac{4 \pi d N}{d \Omega_{\vec{n}}}=\left[\frac{N_{c}}{C_{F}} \widehat{(13)}+\widehat{(24)}+\frac{N_{c}}{4 C_{F}}\left(\hat{D}_{s t}+\hat{D}_{u t}\right)\right] \frac{C_{F}}{N_{c}} \cdot N_{g}^{\prime}
$$

- $g_{1}+g_{2} \rightarrow g_{3}+g_{4}$

$$
\frac{4 \pi d N}{d \Omega_{\vec{n}}}=\left[\widehat{(13)}+\widehat{(24)}+\frac{1}{4}\left(\hat{D}_{s t}+\hat{D}_{u t}\right)\right] \cdot N_{g}^{\prime}
$$

- $g_{1}+q_{2} \rightarrow q_{3}+g_{4}$

$$
\frac{4 \pi d N}{d \Omega_{\vec{n}}}=\left[\widehat{(23)}+\frac{N_{c}}{C_{F}} \widehat{(14)}+\frac{N_{c}}{2 C_{F}}\left(\hat{D}_{t u}+\frac{1}{2 N_{c} C_{F}} \hat{D}_{u s}\right)\right] \frac{C_{F}}{N_{c}} \cdot N_{g}^{\prime}
$$




\section{Bibliography}

1. Yu.L. Dokshitzer, V.A. Khoze and S.I. Troyan. Coherence and physics of QCD jets. In A.H. Mueller, editor, Perturbative QCD, page 241. World Scientific, Singapore, 1989.

2. Yu.L. Dokshitzer, V.A. Khoze, A.H. Mueller and S.I. Troyan. Rev. Mod. Phys., 60:373, 1988.

3. R.K. Ellis. FERMILAB-Conf-87/108-T, 1987.

4. R.K. Ellis. In R. Kotthaus and J. Kühn, editors, Proceedings of the 24th International Conference on High Energy Physics, page 48. SpringerVerlag, Berlin, 1989.

5. R.K. Ellis and W.J. Stirling. FERMILAB-Conf-90/164-T, 1990.

6. R.K. Ellis, G. Marchesini and B.R. Webber. Nucl. Phys., B286:643, 1987. Erratum quoted ibid., B294:1180, 1987.

7. G. Marchesini and B.R. Webber. Nucl. Phys., B310:461, 1988.

8. E. Eichten, I. Hinchliffe, K. Lane and C. Quigg. Rev. Mod. Phys., 56:579, 1984.

9. R.K. Ellis and J.C. Sexton. Nucl. Phys., B282:642, 1987.

10. G. Marchesini and B.R. Webber. Nucl. Phys., B330:261, 1990.

11. Yu.L. Dokshitzer, V.A. Khoze and S.I. Troyan. In M. Derrick, editor, Proceedings of the 6th Int. Conf. on Physics in Collisions, page 417. World Scientific, Singapore, 1987.

12. B.L. Combridge. Nucl. Phys., B151:429, 1979.

13. M. Gluck, J.F. Owens and E. Reya. Phys. Rev., D17:2324, 1978.

14. B. Bambach et al. QCD Generators at LEP. In G. Altarelli, R. Kleiss, and C. Verzegnassi, editors, Proceedings of the Workshop on $Z$ physics at LEP, CERN Report 89-08, volume 3, page 43, 1989. 


\section{Index}

angular ordering, 114, 117,125,

134, 160, 163, 208

strict in MLLA, 117, 194,199

strong in DLA, 144, 203, 206

formal proof, 154

anomalous dimension, 51, 55, 169,

189, 190, 223, 237, 241]

MLLA, 190, 224, 225

monsters, 195, 219

multiplicity, 245

antenna pattern, 310 351

$q \bar{q}, 138$

$q \bar{q} g, 317$

gluon fusion, 343

heavy quarks, 340

quark scattering, 331, 358

$\mathrm{W}$ production, 339

$\mathrm{Z}$ and $\mathrm{H}, 343$

BCM Ansatz, 199

Bethe-Salpeter equations, 158

DLA, 162
LLA, 44, 46, 48 solution, 53

MLLA, 195

Bjorken scaling, 11, 16]

blanching, 12, 14

Born approximation, 25, 37, 41, 156

Born term, 44, 163, 198, 213

bremsstrahlung, 5, 6. 8, 12, 125.

137, 144, 310, 323, 324, 354,

cone, 119, 219, 280, 324, 326

factor, 152, 201]

in initial state, 117

off heavy quarks, 121

theorem, 158

Chudakov effect, 116

coefficient function, 19, 51, 190

DLA, 226]

LLA, 55, 56

MLLA, 191, 218, 243

next-to-MLLA, 208 
next-to-next-to-MLLA, 213

coherence in gluon emission, 114 .

117, 123, 179, 235, 314, 315, 322, 323

in initial state, 120, 134]

interjet, 12, 114, 281, 299, 306,

324, 353

intrajet, 114, 144, 299, 353

in $\mathrm{MC}, 117$

collective phenomena, 281, 297. 299, 305, 314, 316, 322, 328, 353, 354,

color

dipole, 206, 216, 217, 219, 358

factor, 49, 152, 153, 195, 214

flow, 132, 311, 316, 342, 344

transfer, 119, 122, 137, 324, 326

color charge, 4, 8, 13, 15, 32, 33,

117, 131, 137, 156, 163,

183, 214, 217, 280, 291,

325, 327

color monsters, 195, 214,217

conformal invariance, 51

constituent, 93, 95.

mass, $[7$

model, 81, 83, 87

quark, 83,85

counting rule, 93

cumulants, 236, 237, 241 cutoff $Q_{0}$, 165, 169, 181, 196, 197. 222, 229, 232, 250, 254. 290, 292

cutting parameter in nuclear shadowing, 272, 273

DGLAP, see evolution equations

dipole

corrections, 204, 213, 217, 218 scheme, 200, 201

DIS, 22, 24, 83, 118, 136, 262, 264, accompanying radiation, 134 in field theory, 16, 18 on nuclei, 260, 273 spin dependent, 24, 66, 85, final states, [72, [77] subtlety, [75]

dispersion of multiplicity, 184 spectra, 236, 240

dispersion relation, 39, 158

distorted Gaussian, 236, 241, 254

DLA, 144 multigluon production amplitude, 144, 152, 158 cross section, 155, 159

drag effect, 12, 281, 310, 315, 322, 323, 328, 354, heavy Q, 306 
Drell-Yan process, 118

EMC (energy-multiplicity correlation), 132] 247, 282, 284, 295, 314,

EMC effect, 266, 274]

evolution equations, 18, 20, 43,

DGLAP, 48

DLA, 191

MLLA, 194, 196, 197, 222, 225 corrections, 202 exact solution, 225, 235, 253

Fock states, 40, 78, 80, 81, 96

form factor

double logarithmic, 45, 283

elastic, 99 .

nucleon, 101

pion, 99, 100,

probabilistic interpretation, 39,

41, 42, 45, 196

suppression, 98, 177, 196, 287

formation time, 10, 14, 107, 114.

[121]

gauge choice, 32, 146, 202

axial, 32, 35, 58, 67, 778, 215, 216, 219

Feynman, 30, 32

planar, 146, 147, 155, 203, 216.
Generating Functional (GF), 158, 160, 163, 165, 170, 178, 189, 197, 202

DLA, 161, 167]

MLLA, 195, 197,

gluer, 13.

Gribov's gap, 136

hadronization time, 10

Higgs production accompanying radiation, 119]

hump-backed plateau, 123, 235, 246, 354

DLA, 179

MLLA, 229]

jet calculus, 158

Kinoshita-Lee-Nauenberg th., 35

$\mathrm{KNO}$ distribution, 171, 174, 176, 177 scaling, 144, 170]

kurtosis, 236]

Landshoff scattering picture, 91 . 93, 99, 103

large- $N_{c}$ limit, 200, 310, 316, 324, 344, 348, 354, 357

limiting parton density, 264, 271] 
limiting spectrum, 232, 234, 250, 251 multiplicity, 234, 249, 250, 336 next-to-MLLA shift, 245 shape, 237, 239

LLA, 17

LPHD, 230, 232, 249, 251, 254, 256, 280, 301, 315, 323.

MLLA, 189

Monte Carlo scheme, 117, 128, 316, 344, 354 dipole, 200

HERWIG, 117 independent fragmentation, 15 LUND, 311, 331, 346, 348

multiplicity, 9, 125, 126, 168, 169,

172, 184, 188, 192, 218,

231, 249, 285, 301]

collimation, 293

correlators, 166, 167, 171]

flow, 12, 131, 280, 283, 294, 299, 326

fluctuations, 144, 165, 177

in jet ensembles, 281, 282

qqg, 294, 301,

MLLA, 233, 234, 302

of hadrons, 249, 336

of heavy quarks, 250] ratio, 52, 243, 245, 305,

one-loop approximation, 26

OPE, 19, 20, 55, 66, 73, 75, 78, 83

opening angle, 117, 125, 158, 161,

163, 166, 180, 222, 280.

as an evolution parameter, 190.

193, 196, 285, 336]

restricted, 246]

shrinkage, 280, 286

parton

form factor, 12, 37, 157

DLA, 157, 164

MLLA, 196, 198

ladder, 28

saturation, 264, 267, 269, 271]

parton cascades, 11, 15, 116, 144, 159, 246, 249, 256, 280, 285, 324, 353

angular ordered, 117, 125, 285,

cascading factor, 296, 331]

developed, 232, 235, 312

DLA, 178, 203

MLLA, 189, 199, 208, 242

multiplicity, 234

simulation, 128, 200

space-like, 117, 134

truncated, 250, 254 
parton model, 2, 19, 20, 66, 83, 110,

124, 260, 264, 266, 269

QCD improved, 20]

parton splitting, 44

functions, 48, 193, 194

physical gauge, 32, 33, 42, 69, 108,

146, 147.

renormalization program, 35

preconfinement, 231

rapidity plateau, 13, 326

regeneration time, 5, 6]

Regge theory, 2, 57.

renormalization group, 19, 55, 193, 225

running coupling, 42, 168, 169, 173,

194, 234, 256

and confinement, 11

in QED, 37, 39.

MLLA, 192, 225

one-loop, 47

scaling violation, 11, 35, 254

shadowing, 260, 262, 269, 273, 274

in initial distributions, 273

shower, 117, 144, 150, 189, 190, 193.

QED, 114

skewness, 236, 241, 242

Skyrme model, 87
SL (single-logarithmic effects, corrections), 117. 189, 193. 194, 225, 239, 241,

soft insertion rules, 153, 202

string

effect, 129, 281, 311, 315,

model, 4, 311, 312, 315, 331,

343, 346, 348, 352, 353

structure functions, 18, 24, 274

Sudakov form factor, 44, 96

Sudakov variables, 24

super-symmetry, 50, 51, 58, 328

transparency, 102104

twist, 19, 20, 51, 69

V-scheme, 194, 195, 204, 208, 212,

213 


\section{abbreviations}

- DIS - deep inelastic (lepton-hadron) scattering

- DGLAP - Dokshitzer-Gribov-Lipatov-Altarelli-Parisi (evolution equations)

- DLA - double logarithmic approximation

- GF - generating functional

- KNO - Koba-Nielsen-Olesen (scaling)

- LLA - leading logarithmic approximation

- LPHD - local parton-hadron duality

- MLLA - modified leading logarithmic approximation

- PT - perturbative

- RG - renormalization group

- SL - single logarithmic (corrections, effects) 